\title{
Deformation Quantization for Actions of Kählerian Lie Groups
}

\author{
Pierre Bieliavsky \\ Victor Gayral \\ University of Louvain, Belgium \\ E-mail address: pierre.bieliavsky@gmail.com \\ University of Reims, France \\ E-mail address: victor gayral@univ-reims.fr
}


2010 Mathematics Subject Classification. Primary 22E30, 46L87, 81R60, 58B34, 81R30, 53C $35,32 \mathrm{M} 15,53 \mathrm{D} 55$

Key words and phrases. Strict deformation quantization, Symmetric spaces, Representation theory of Lie groups, Deformation of $C^{*}$-algebras, Symplectic Lie groups, Coherent states, Noncommutative harmonic analysis, Noncommutative geometry

Work supported by the Belgian Interuniversity Attraction Pole (IAP) within the framework "Dynamics, Geometry and Statistical Physics" (DYGEST).

Abstract. Let $\mathbb{B}$ be a Lie group admitting a left-invariant negatively curved Kählerian structure. Consider a strongly continuous action $\alpha$ of $\mathbb{B}$ on a Fréchet algebra $\mathcal{A}$. Denote by $\mathcal{A}^{\infty}$ the associated Fréchet algebra of smooth vectors for this action. In the Abelian case $\mathbb{B}=\mathbb{R}^{2 n}$ and $\alpha$ isometric, Marc Rieffel proved in 26 that Weyl's operator symbol composition formula (the so called Moyal product) yields a deformation through Fréchet algebra structures $\left\{\star_{\theta}^{\alpha}\right\}_{\theta \in \mathbb{R}}$ on $\mathcal{A}^{\infty}$. When $\mathcal{A}$ is a $C^{*}$-algebra, every deformed Fréchet algebra $\left(\mathcal{A}^{\infty}, \star_{\theta}^{\alpha}\right)$ admits a compatible pre- $C^{*}$-structure, hence yielding a deformation theory at the level of $C^{*}$-algebras too. In this memoir, we prove both analogous statements for general negatively curved Kählerian groups. The construction relies on the one hand on combining a non-Abelian version of oscillatory integral on tempered Lie groups with geometrical objects coming from invariant WKBquantization of solvable symplectic symmetric spaces, and, on the second hand, in establishing a non-Abelian version of the Calderón-Vaillancourt Theorem. In particular, we give an oscillating kernel formula for WKB-star products on symplectic symmetric spaces that fiber over an exponential Lie group. 


\section{Warning}

The published version of the present article contains a mistake which invalidates the proof of the invariance of the $K$-theory under the deformation (Theorem 8.50 in the printed version and Theorem 7.50 in the former arXiv version). The mistake is located in Proposition 8.47 (Proposition 7.47 in the former arXiv version), where it is claimed that the map $\mathbb{R}^{2} \rightarrow \mathbb{R}^{2},\left(a_{1}, a_{2}\right) \mapsto\left(e^{-2 a_{2}} \sinh \left(2 a_{1}\right),-e^{-2 a_{1}} \sinh \left(2 a_{2}\right)\right)$ is diffeomorphism. In fact, this map only defines a diffeomorphism from $\mathbb{R}^{2}$ onto a proper open subspace of $\mathbb{R}^{2}$. As a consequence, the deformed $C^{*}$-algebra $A_{\theta, \mathbf{m}}$ needs not to be Strongly Morita equivalent to the reduced crossed-product $\mathbb{B} \ltimes\left(\mathcal{K}\left(\mathcal{H}_{\chi}\right) \otimes A\right)$ and the $K$-theory needs not to be an invariant of the deformation.

In this updated version, we have removed the whole contents of Section 7.7 (Section 8.7 of the published version). 



\section{Contents}

Warning iii

Introduction 1

$\begin{array}{ll}\text { Notations and conventions } & 7\end{array}$

Chapter 1. Oscillatory integrals 9

1.1. Symbol spaces 10

1.2. Tempered pairs 21

1.3. An oscillatory integral for admissible tempered pairs 29

1.4. A Fubini Theorem for semi-direct products 32

1.5. A Schwartz space for tempered pairs 34

1.6. Bilinear mappings from the oscillatory integral 36

Chapter 2. Tempered pairs for Kählerian Lie groups 41

2.1. Pyatetskii-Shapiro's theory 41

2.2. Geometric structures on elementary normal $\mathbf{j}$-groups 43

2.3. Tempered pair for elementary normal $\mathbf{j}$-groups 46

2.4. Tempered pairs for normal $\mathbf{j}$-groups 51

Chapter 3. Non-formal star-products 57

3.1. Star-products on normal $\mathbf{j}$-groups $\quad 57$

3.2. An oscillatory integral formula for the star-product 60

Chapter 4. Deformation of Fréchet algebras 65

4.1. The deformed product 65

4.2. Relation with the fixed point algebra 71

4.3. Functorial properties of the deformed product 72

Chapter 5. Quantization of polarized symplectic symmetric spaces 77

5.1. Polarized symplectic symmetric spaces $\quad 79$

5.2. Unitary representations of symmetric spaces 84

5.3. Locality and the one-point phase 88

5.4. Unitarity and midpoints for elementary spaces 89

5.5. The $\star$-product as the composition law of symbols 94

5.6. The three-point kernel 96

5.7. Extensions of polarization quadruples 99

Chapter 6. Quantization of Kählerian Lie groups 103

6.1. The transvection quadruple of an elementary normal $\mathbf{j}$-group $\quad 103$

6.2. Quantization of elementary normal $\mathbf{j}$-groups 108

6.3. Quantization of normal j-groups 
Chapter 7. Deformation of $C^{*}$-algebras

7.1. Wavelet analysis

7.2. A tempered pair from the one-point phase

7.3. Extension of the oscillatory integral

7.4. A Calderón-Vaillancourt type estimate

7.5. The deformed $C^{*}$-norm

7.6. Functorial properties of the deformation 


\section{Introduction}

The general idea of deforming a given theory by use of its symmetries goes back to Drinfel'd. One paradigm being that the data of a Drinfel'd twist based on a bialgebra acting on an associative algebra $\mathbb{A}$, produces an associative deformation of $\mathbb{A}$. In the context of Lie theory, one considers for instance the category of module-algebras over the universal enveloping algebra $\mathcal{U}(\mathfrak{g})$ of the Lie algebra $\mathfrak{g}$ of a given Lie group $G$. In that situation, the notion of Drinfel'd twist is in a one to one correspondence with the one of left-invariant formal star-product $\star_{\nu}$ on the space of formal power series $C^{\infty}(G)[[\nu]]$, see [14. Disposing of such a twist, every $\mathcal{U}(\mathfrak{g})$-module-algebra $\mathbb{A}$ may then be formally deformed into an associative algebra $\mathbb{A}[[\nu]]$. It is important to observe that, within this situation, the symplectic leaf $\mathbb{B}$ through the unit element $e$ of $G$ in the characteristic foliation of the (left-invariant) Poisson structure directing the star-product $\star_{\nu}$, always consists of an immersed Lie subgroup of $G$. The Lie group $\mathbb{B}$ therefore carries a left-invariant symplectic structure. This stresses the importance of symplectic Lie groups (i.e. connected Lie groups endowed with invariant symplectic forms) as semi-classical approximations of Drinfel'd twists attached to Lie algebras.

In the present memoir, we address the question of designing non-formal Drinfel'd twists for actions of symplectic Lie groups $\mathbb{B}$ that underly negatively curved Kählerian Lie groups, i.e. Lie groups that admit a left-invariant Kählerian structure of negative curvature. These groups exactly correspond to the normal $\mathbf{j}$-algebras defined by Pyatetskii-Shapiro in his work on automorphic forms $\mathbf{2 2}$. In particular, this class of groups contains all Iwasawa factors $A N$ of Hermitian type simple Lie groups $G=K A N$.

Roughly speaking, one looks for a smooth one-parameter family of complex valued smooth two-point functions on the group, $\left\{K_{\theta}\right\}_{\theta \in \mathbb{R}} \subset C^{\infty}(\mathbb{B} \times \mathbb{B}, \mathbb{C})$, with the property that, for every strongly continuous and isometric action $\alpha$ of $\mathbb{B}$ on a $C^{*}$-algebra $A$, the following formula

$$
a \star_{\theta}^{\alpha} b:=\int_{\mathbb{B} \times \mathbb{B}} K_{\theta}(x, y) \alpha_{x}(a) \alpha_{y}(b) \mathrm{d} x \mathrm{~d} y,
$$

defines a one-parameter deformation of the $C^{*}$-algebra $A$.

The above program was realized by Marc Rieffel in the particular case of the Abelian Lie group $\mathbb{B}=\mathbb{R}^{2 n}$ in [26. More precisely, Rieffel proved that for any strongly continuous and isometric action of $\mathbb{R}^{2 n}$ on any Fréchet algebra $\mathcal{A}$, the associated Fréchet subalgebra $\mathcal{A}^{\infty}$ of smooth vectors for this action, is deformed by the rule (0.1), where the two-point kernel there, consists of the Weyl symbol composition kernel:

$$
K_{\theta}(x, y):=\theta^{-2 n} \exp \left\{\frac{i}{\theta} \omega^{0}(x, y)\right\},
$$


associated to a translation invariant symplectic structure $\omega^{0}$ on $\mathbb{R}^{2 n}$. The associated star-product therefore corresponds here to Moyal's product. In the special case where the Fréchet algebra $\mathcal{A}$ is a $C^{*}$-algebra, Rieffel also constructed a deformed $C^{*}$-structure, so that $\left(\mathcal{A}^{\infty}, \star_{\theta}^{\alpha}\right)$ becomes a pre- $C^{*}$-algebra, which in turn yields a deformation theory at the level of $C^{*}$-algebras too. Many further results have been proved then (for example continuity of the field of deformed $C^{*}$-algebras [26], invariance of the $K$-theory $\mathbf{2 7} \ldots$ ), and many applications have been found (for instance in locally compact quantum groups [28], quantum fields theory [9, 10, spectral triples $\mathbf{1 5} \ldots)$.

In this memoir, we investigate the deformation theory of $C^{*}$-algebras endowed with an isometric action of a negatively curved Kählerian Lie group. Most of the results we present here are of a pure analytical nature. Indeed, once a family $\left\{K_{\theta}\right\}_{\theta \in \mathbb{R}}$ of associative (i.e. such that the associated deformed product (0.1) is at least formally associative) two-point functions has been found, in order to give a precise meaning to the associated multiplication rule, there is no doubt that the integrals in (0.1) need to be interpreted in a suitable (here oscillatory) sense. Indeed, there is no reason to expect the two-point function $K_{\theta}$ to be integrable: it is typically not even bounded in the non-Abelian case! Thus, already in the case of an isometric action on a $C^{*}$-algebra, we have to face a serious analytical difficulty. We stress that contrarily to the case of $\mathbb{R}^{2 n}$, in the situation of a non-Abelian group action, this is an highly non-trivial feature of our deformation theory.

The memoir is organized as follows.

In chapter 1, we start by introducing non-Abelian and unbounded versions of Fréchet-valued symbol spaces on a Lie group $G$, with Lie algebra $\mathfrak{g}$ :

$$
\mathcal{B} \underline{\mu}(G, \mathcal{E}):=\left\{f \in C^{\infty}(G, \mathcal{E}): \forall X \in \mathcal{U}(\mathfrak{g}), \forall j \in \mathbb{N}, \exists C>0:\|\widetilde{X} f\|_{j} \leq C \mu_{j}\right\},
$$

where $\mathcal{E}$ is a Fréchet space, $\underline{\mu}:=\left\{\mu_{j}\right\}_{j \in \mathbb{N}}$ is a countable family of specific positive functions on $G$, called weights (see Definition 1.1) affiliated to a countable set of semi-norms $\left\{\|\cdot\|_{j}\right\}_{j \in \mathbb{N}}$ defining the Fréchet topology on $\mathcal{E}$ and where $\tilde{X}$ is the left-invariant differential operator on $G$ associated to an element $X \in \mathcal{U}(\mathfrak{g})$. For example, $\mathcal{B}^{1}(G, \mathbb{C})$ consists of the smooth vectors for the right regular representation of $G$ on the space of bounded right-uniformly continuous functions on $G$ (the uniform structures on $G$ are generally not balanced in our non-Abelian situation) and it coincides with Laurent Schwartz's space $\mathcal{B}$ when $G=\mathbb{R}^{n}$. We shall also mention that function spaces on Lie groups of a similar type are considered in $\mathbf{3 0}$ and in $\left[\mathbf{2 0}\right.$ in the context of actions of $\mathbb{R}^{d}$ on locally convex algebras.

We then define a notion of oscillatory integrals on Lie groups $G$ that are endowed with a specific type of smooth function $S \in C^{\infty}(G, \mathbb{R})$ (see Definitions 1.17 1.22 and 1.24). We call such a pair $(G, S)$ an admissible tempered pair. The main result of this chapter is that associated to an admissible tempered pair $(G, S)$, and given a growth-controlled function $\mathbf{m}$, the oscillatory integral

$$
\mathcal{D}(G, \mathcal{E}) \rightarrow \mathcal{E}, \quad F \mapsto \int_{G} \mathbf{m} e^{i S} F,
$$

canonically extends from $\mathcal{D}(G, \mathcal{E})$, the space of smooth compactly supported functions, to our symbol space $\mathcal{B}-\underline{\mu}(G, \mathcal{E})$. This construction is explained in Definition 
1.31, which turns out to apply in our situation as a direct consequence of Proposition 1.29, the main technical result of this chapter.

In chapter 2 , we consider an arbitrary normal $\mathbf{j}$-group $\mathbb{B}$ (i.e. a connected simply connected Lie group whose Lie algebra is a normal $\mathbf{j}$-algebra - see Definition 2.1). The main result of this chapter, Theorem 2.35, shows that its square $\mathbb{B} \times \mathbb{B}$ canonically underlies an admissible tempered pair $\left(\mathbb{B} \times \mathbb{B}, S_{\text {can }}^{\mathbb{B}}\right)$. When elementary, every normal $\mathbf{j}$-group has a canonical simply transitive action on a specific solvable symplectic symmetric space. The two-point function $S_{\text {can }}^{\mathbb{B}}$ we consider here comes from an earlier work of one of us. It consists of the sum of the phases $S_{\text {can }}^{\mathbb{S}_{j}}$ of the oscillatory kernels associated to invariant star-products on solvable symplectic symmetric space [3, 8, in the Pyatetskii-Shapiro decomposition [22] of a normal $\mathbf{j}$-group $\mathbb{B}$ into a sequence of split extensions of elementary normal $\mathbf{j}$-factors: $\mathbb{B}=\left(\ldots\left(\left(\mathbb{S}_{1} \ltimes \mathbb{S}_{2}\right) \ltimes \mathbb{S}_{3}\right) \ltimes \ldots\right) \ltimes \mathbb{S}_{N}$. The two-point phase function $S_{\text {can }}^{\mathbb{S}}$ in the elementary case, then consists of the symplectic area of the unique geodesic triangle in $\mathbb{S}$ (viewed as a solvable symplectic symmetric space), whose geodesic edges admit $e, x$ and $y$ as midpoints ( $e$ denotes the unit element of the group $\mathbb{S}$ ):

$$
S_{\text {can }}^{\mathbb{S}}\left(x_{1}, x_{2}\right):=\operatorname{Area}\left(\Phi_{\mathbb{S}}^{-1}\left(e, x_{1}, x_{2}\right)\right),
$$

with

$$
\Phi_{\mathbb{S}}: \mathbb{S}^{3} \rightarrow \mathbb{S}^{3}, \quad\left(x_{1}, x_{2}, x_{3}\right) \mapsto\left(\operatorname{mid}\left(x_{1}, x_{2}\right), \operatorname{mid}\left(x_{2}, x_{3}\right), \operatorname{mid}\left(x_{3}, x_{1}\right)\right),
$$

where $\operatorname{mid}(x, y)$ denotes the geodesic midpoint between $x$ and $y$ in $\mathbb{S}$ (again uniquely defined in our situation).

In chapter 3 , we consider an arbitrary normal $\mathbf{j}$-group $\mathbb{B}$, and define the abovementioned oscillatory kernels $K_{\theta}$ simply by tensorizing oscillating kernels found in 8 ] on elementary $\mathbf{j}$-factors. The resulting kernel has the form

$$
K_{\theta}=\theta^{-\operatorname{dim} \mathbb{B}} \mathbf{m}_{\text {can }}^{\mathbb{B}} \exp \left\{\frac{i}{\theta} S_{\text {can }}^{\mathbb{B}}\right\},
$$

where $S_{\text {can }}^{\mathbb{B}}$ is the two-point phase mentioned in the description of chapter 2 above, and $\mathbf{m}_{\text {can }}^{\mathbb{B}}=\mathbf{m}_{\text {can }}^{\mathbb{S}_{1}} \otimes \cdots \otimes \mathbf{m}_{\text {can }}^{\mathbb{S}_{N}}$, where $\mathbf{m}_{\text {can }}^{\mathbb{S}_{j}}=\operatorname{Jac}_{\Phi_{\mathbb{S}_{j}}^{-1}}^{1 / 2}$ denotes the square root of the Jacobian of the "medial triangle" map $\Phi_{\mathbb{S}}^{-1}$. In particular, it defines an oscillatory integral on every symbol space of the type $\mathcal{B} \underline{\mu}(\mathbb{B} \times \mathbb{B}, \mathcal{B} \underline{\nu}(\mathbb{B}, \mathcal{E}))$. When valued in a Fréchet algebra $\mathcal{A}$, this yields a non-perturbative and associative star-product $\star_{\theta}$ on the union of all symbol spaces $\mathcal{B} \underline{\mu}(\mathbb{B}, \mathcal{A})$.

In chapter 4 , we consider any tempered action of a normal $\mathbf{j}$-group $\mathbb{B}$ on a Fréchet algebra $\mathcal{A}$. By tempered action we mean a strongly continuous action $\alpha$ of $\mathbb{B}$ by automorphisms on $\mathcal{A}$, such that for every semi-norm $\|\cdot\|_{j}$ there is a weight ("tempered" for a suitable notion of temperedness) $\mu_{j}^{\alpha}$ such that $\left\|\alpha_{g}(a)\right\|_{j} \leq$ $\mu_{j}^{\alpha}(g)\|a\|_{j}$ for all $a \in \mathcal{A}$ and $g \in \mathbb{B}$. In that case, the space of smooth vectors $\mathcal{A}^{\infty}$ for $\alpha$ naturally identifies with a subspace of $\mathcal{B} \underline{\hat{\mu}}\left(\mathbb{B}, \mathcal{A}^{\infty}\right.$ ) (where $\underline{\hat{\mu}}$ is affiliated to $\left.\underline{\mu}^{\alpha}=\left\{\mu_{j}^{\alpha}\right\}_{j \in \mathbb{N}}\right)$ through the injective map:

$$
\alpha: \mathcal{A}^{\infty} \rightarrow \mathcal{B} \underline{\hat{\mu}}\left(\mathbb{B}, \mathcal{A}^{\infty}\right), \quad a \mapsto\left[g \mapsto \alpha_{g}(a)\right] .
$$

We stress that even in the case of an isometric action, and contrarily to the Abelian situation, the map $\alpha$ always takes values in a symbol space $\mathcal{B}=$, for a non-trivial 
sequence of weights $\mu$. This explains why the non-Abelian framework forces to consider such symbol spaces. Applying the results of chapter 3 to this situation, we get a new associative product on $\mathcal{A}^{\infty}$ defined by the formula

$$
a \star_{\theta}^{\alpha} b:=\left(\alpha(a) \star_{\theta} \alpha(b)\right)(e) .
$$

Then, the main result of this chapter, stated as Theorem 4.8 in the text, is the following fact:

\section{Universal Deformation Formula for Actions of Kählerian Lie Groups on Fréchet Algebras: \\ Let $(\mathcal{A}, \alpha, \mathbb{B})$ be a Fréchet algebra endowed with a tempered action of a normal $\mathbf{j}$-group. Then, $\left(\mathcal{A}^{\infty}, \star_{\theta}^{\alpha}\right)$ is an associative Fréchet algebra, (abusively) called the Fréchet deformation of $\mathcal{A}$.}

Following the terminology introduced in [17, the word "universal" refers to the fact that our deformation procedure applies to any Fréchet algebra the Kählerian Lie group acts on. The word "universal" does not refer to the possibility that our construction might yield all such deformation procedures valid for a given Kählerian Lie group. However, regarding the last sentence, the following remark can nevertheless be made. In order to get rid of technicalities let us consider, for a short moment, the purely formal framework of formal Drinfel'd twists based on the bialgebra underlying the enveloping algebra $\mathcal{U}(\mathfrak{b})$ of the Lie group $\mathbb{B}$. Given such a Drinfel'd twist $F \in \mathcal{U}(\mathfrak{b}) \otimes \mathcal{U}(\mathfrak{b})[[\nu]]$, one defines the internal symmetry of the twist as the group $G(F)$, consisting of diffeomorphisms that preserve the twist:

$$
G(F):=\left\{\varphi: \mathbb{B} \rightarrow \mathbb{B} \mid \varphi_{\star} \tilde{F}=\tilde{F}\right\},
$$

where $\tilde{F}$ denotes the left-invariant (formal sequence of) operator(s) associated to the twist $F$. Within the present work we treat (all) the situations where, in the elementary normal case, the internal symmetry equals the automorphism group of a symmetric symplectic space structure on $\mathbb{B}$ whose underlying affine connection consists in the canonical torsion free invariant connection on the group $\mathbb{B}$ (see [3]).

The rest of the memoir is devoted to the construction of a pre- $C^{*}$-structure on $\left(\mathcal{A}^{\infty}, \star_{\theta}^{\alpha}\right)$, in the case $\mathcal{A}$ is a $C^{*}$-algebra. The method we use is a generalization of Unterberger's Fuchs calculus $\mathbf{3 3}$ and fits within the general framework of "Moyal quantizer" as defined in [12] (see also [18, Section 3.5]).

In chapter 5, we define a special class of symplectic symmetric spaces which naturally give rise to explicit WKB-quantizations (i.e. invariant star-products representable through oscillatory kernels) that underlie pseudo-differential operator calculus. Roughly speaking, an elementary symplectic symmetric space is a symplectic symmetric space that consists of the total space of a fibration in flat fibers over a Lie group $Q$ of exponential type. In that case, a variant of Kirillov's orbit method yields a unitary and self-adjoint representation on an Hilbert-space $\mathcal{H}$, of the symmetric space $M$ :

$$
\Omega: M \rightarrow \mathcal{U}_{\mathrm{sa}}(\mathcal{H})
$$


with associated "quantization rule":

$$
\Omega: L^{1}(M) \rightarrow \mathcal{B}(\mathcal{H}), \quad F \mapsto \Omega(F):=\int_{M} F(x) \Omega(x) \mathrm{d} x
$$

Weighting the above mapping by the multiplication by a (growth controlled) function $\mathbf{m}$ defined on the base $Q$ yields a pair of adjoint maps:

$$
\Omega_{\mathbf{m}}: L^{2}(M) \rightarrow \mathcal{L}^{2}(\mathcal{H}) \text { and } \sigma_{\mathbf{m}}: \mathcal{L}^{2}(\mathcal{H}) \rightarrow L^{2}(M)
$$

where $\mathcal{L}^{2}(\mathcal{H})$ denotes the Hilbert space of Hilbert-Schmidt operators on $\mathcal{H}$. Both of the above maps are equivariant under the whole automorphism group of $M$. Note that this last feature very much contrasts with the usual notion of coherentstate quantization for groups (as opposed to symmetric spaces). The corresponding "Berezin transform" $B_{\mathbf{m}}:=\sigma_{\mathbf{m}} \circ \Omega_{\mathbf{m}}$ is explicitly controlled. In particular, when invertible, the associated star-product $F_{1} \star F_{2}:=B_{\mathbf{m}}^{-1} \sigma_{\mathbf{m}}\left(\Omega_{\mathbf{m}}\left(F_{1}\right) \Omega_{\mathbf{m}}\left(F_{2}\right)\right)$ is of oscillatory (WKB) type and its associated kernel is explicitly determined. Note that, because entirely explicit, this chapter yields a proof of Weinstein's conjectural form for star-product WKB-kernels on symmetric spaces [36 in the situation considered here. The chapter ends with considerations on extending the construction to semi-direct products.

Chapter 6 is entirely devoted to applying the construction of chapter 5 to the particular case of Kählerian Lie groups with negative curvature. Such a Lie group is always a normal $\mathbf{j}$-group is the sense of Pyatetskii-Shapiro. Each of its elementary factors admits the structure of an elementary symplectic symmetric space. Accordingly to [3], the obtained non-formal star products coincide with the one described in chapters 2-4.

Chapter 7 deals with the deformation theory for $C^{*}$-algebras. We eventually prove the following statement:

\section{Universal Deformation Formula for Actions of Kählerian Lie Groups on $C^{*}$-Algebras: \\ Let $(A, \alpha, \mathbb{B})$ be a $C^{*}$-algebra endowed with a strongly continuous and isometric action of a normal $\mathbf{j}$-group. Then, there exists a canonical $C^{*}$-norm on the Fréchet algebra $\left(A^{\infty}, \star_{\theta}^{\alpha}\right)$. Its $C^{*}$-closure is (abusively) called the $C^{*}$-deformation of $A$.}

The above statement follows from a non-Abelian generalization of the CalderónVaillancourt Theorem in the context of the usual Weyl pseudo-differential calculus on $\mathbb{R}^{2 n}$ (see Theorem 7.20$)$. Our result asserts that the element $\Omega_{\mathbf{m}}(F)$ associated to a function $F$ in $\mathcal{B}^{1}(\mathbb{B}, A)$ naturally consists of an element of the spatial tensor product of $A$ by $\mathcal{B}(\mathcal{H})$. Its proof relies on a combination of a resolution of the identity obtained from wavelet analysis considerations (see section 7.1) and further properties of our oscillatory integral defined in chapter 1. We finally prove that the $K$-theory is an invariant of the deformation.

Acknowledgments We warmly thank the referee for his positive criticism. His comments, remarks and suggestions have greatly improved this work. 



\section{Notations and conventions}

Given a Lie group $G$, with Lie algebra $\mathfrak{g}$, we denote by $\mathrm{d}_{G}(g)$ a left invariant Haar measure. In the non-unimodular case, we consider the modular function $\Delta_{G}$, defined by the relation:

$$
\mathrm{d}_{G}(g) \Delta_{G}(g):=\mathrm{d}_{G}\left(g^{-1}\right) .
$$

Unless otherwise specified, $L^{p}(G), p \in[1, \infty]$, will always denote the Lebesgue space associated with the choice of a left-invariant Haar measure made above. We also denote by $\mathcal{D}(G)$ the space of smooth compactly supported functions on $G$ and by $\mathcal{D}^{\prime}(G)$ the dual space of distributions.

We use the notations $L^{\star}$ and $R^{\star}$, for the left and right regular actions:

$$
L_{g}^{\star} f\left(g^{\prime}\right):=f\left(g^{-1} g^{\prime}\right), \quad R_{g}^{\star} f\left(g^{\prime}\right):=f\left(g^{\prime} g\right) .
$$

By $\widetilde{X}$ and $\underline{X}$, we mean the left-invariant and right-invariant vector fields on $G$ associated to the elements $X$ and $-X$ of $\mathfrak{g}$ :

$$
\widetilde{X}:=\left.\frac{d}{d t}\right|_{t=0} R_{e^{t X}}^{\star}, \quad \underline{X}:=\left.\frac{d}{d t}\right|_{t=0} L_{e^{t X}}^{\star} .
$$

Given a element $X$ of the universal enveloping algebra $\mathcal{U}(\mathfrak{g})$ of $\mathfrak{g}$, we adopt the same notations $\widetilde{X}$ and $\underline{X}$ for the associated left- and right-invariant differential operators on $G$. More generally, if $\alpha$ is an action of $G$ on a topological vector space $\mathcal{E}$, we consider the infinitesimal form of the action, given for $X \in \mathfrak{g}$ by:

$$
X^{\alpha}(a):=\left.\frac{d}{d t}\right|_{t=0} \alpha_{e^{t X}}(a), \quad a \in \mathcal{E}^{\infty},
$$

and extended to the whole universal enveloping algebra $\mathcal{U}(\mathfrak{g})$, by declaring that the $\operatorname{map} \mathcal{U}(\mathfrak{b}) \rightarrow \operatorname{End}\left(\mathcal{A}^{\infty}\right), X \mapsto X^{\alpha}$ is an algebra homomorphism. Here, $\mathcal{E}^{\infty}$ denotes the set of smooth vectors for the action:

$$
\mathcal{E}^{\infty}:=\left\{a \in \mathcal{E}:\left[g \mapsto \alpha_{g}(a)\right] \in C^{\infty}(G, \mathcal{E})\right\} .
$$

Let $\Delta_{\mathcal{U}}$ be the ordinary co-product of $\mathcal{U}(\mathfrak{g})$. We make use of the Sweedler notation:

$$
\Delta_{\mathcal{U}}(X)=\sum_{(X)} X_{(1)} \otimes X_{(2)} \in \mathcal{U}(\mathfrak{g}) \otimes \mathcal{U}(\mathfrak{g}), \quad X \in \mathcal{U}(\mathfrak{g})
$$

and accordingly, for $f_{1}, f_{2} \in C^{\infty}(G)$ and $X \in \mathcal{U}(\mathfrak{g})$, we write

$$
\tilde{X}\left(f_{1} f_{2}\right)=\sum_{(X)}\left(\tilde{X}_{(1)} f_{1}\right)\left(\tilde{X}_{(2)} f_{2}\right), \quad \underline{X}\left(f_{1} f_{2}\right)=\sum_{(X)}\left(\underline{X}_{(1)} f_{1}\right)\left(\underline{X}_{(2)} f_{2}\right) .
$$


More generally, we use the notation

$$
\begin{aligned}
\left(\Delta_{\mathcal{U}} \otimes \mathrm{Id}\right) \circ \Delta_{\mathcal{U}}(X) & =\sum_{(X)} \sum_{\left(X_{(1)}\right)}\left(X_{(1)}\right)_{(1)} \otimes\left(X_{(1)}\right)_{(2)} \otimes X_{(2)} \\
& =: \sum_{(X)} X_{(11)} \otimes X_{(12)} \otimes X_{(2)}
\end{aligned}
$$

and obvious generalization of it.

To a fixed ordered basis $\left\{X_{1}, \ldots, X_{m}\right\}$ of the Lie algebra $\mathfrak{g}$, we associate a PBW basis of $\mathcal{U}(\mathfrak{g})$ :

$$
\left\{X^{\beta}, \beta \in \mathbb{N}^{m}\right\}, \quad X^{\beta}:=X_{1}^{\beta_{1}} X_{2}^{\beta_{2}} \ldots X_{m}^{\beta_{m}} .
$$

This induces a filtration

$$
\mathcal{U}(\mathfrak{g})=\bigcup_{k \in \mathbb{N}} \mathcal{U}_{k}(\mathfrak{g}), \quad \mathcal{U}_{k}(\mathfrak{g}) \subset \mathcal{U}_{l}(\mathfrak{g}), \quad k \leq l,
$$

in terms of the subsets

$$
\mathcal{U}_{k}(\mathfrak{g}):=\left\{\sum_{|\beta| \leq k} C_{\beta} X^{\beta}, C_{\beta} \in \mathbb{R}\right\}, \quad k \in \mathbb{N},
$$

where $|\beta|:=\beta_{1}+\cdots+\beta_{m}$. For $\beta, \beta_{1}, \beta_{2} \in \mathbb{N}^{m}$, we define the 'structure constants' $\omega_{\beta}^{\beta_{1}, \beta_{2}} \in \mathbb{R}$ of $\mathcal{U}(\mathfrak{g})$, by

$$
X^{\beta_{1}} X^{\beta_{2}}=\sum_{|\beta| \leq\left|\beta_{1}\right|+\left|\beta_{2}\right|} \omega_{\beta}^{\beta_{1}, \beta_{2}} X^{\beta} \in \mathcal{U}_{\left|\beta_{1}\right|+\left|\beta_{2}\right|}(\mathfrak{g}) .
$$

We endow the finite dimensional vector space $\mathcal{U}_{k}(\mathfrak{g})$, with the $\ell^{1}$-norm $|\cdot|_{k}$ within the basis $\left\{X^{\beta},|\beta| \leq k\right\}$ :

$$
|X|_{k}:=\sum_{|\beta| \leq k}\left|C_{\beta}\right| \quad \text { if } \quad X=\sum_{|\beta| \leq k} C_{\beta} X^{\beta} \in \mathcal{U}_{k}(\mathfrak{g}) .
$$

We observe that the family of norms $\left\{|\cdot|_{k}\right\}_{k \in \mathbb{N}}$ is compatible with the filtered structure of $\mathcal{U}(\mathfrak{g})$, in the sense that if $X \in \mathcal{U}_{k}(\mathfrak{g})$, then $|X|_{k}=|X|_{l}$ whenever $l \geq k$. Considering a subspace $V \subset \mathfrak{g}$, we also denote by $\mathcal{U}(V)$ the unital subalgebra of $\mathcal{U}(\mathfrak{g})$ generated by $V$ :

$$
\mathcal{U}(V)=\operatorname{span}\left\{X_{1} X_{2} \ldots X_{n}: X_{j} \in V, n \in \mathbb{N}\right\}
$$

that we may filtrate using the induced filtration of $\mathcal{U}(\mathfrak{g})$. We also observe that the co-product preserves the latter subalgebras, in the sense that $\Delta_{\mathcal{U}}(\mathcal{U}(V)) \subset$ $\mathcal{U}(V) \otimes \mathcal{U}(V)$.

Regarding the uniform structures on a locally compact group $G$, we say that a function $f: G \rightarrow \mathbb{C}$ is right (respectively left) uniformly continuous if for all $\varepsilon>0$, there exists $U$, an open neighborhood of the neutral element $e$, such that for all $(g, h) \in G \times G$ we have

$$
\left.|f(g)-f(h)| \leq \varepsilon, \quad \text { whenever } \quad g^{-1} h \in U \quad \text { (respectively } h g^{-1} \in U\right) .
$$

We call a Lie group $G$ (with Lie algebra $\mathfrak{g}$ ) exponential, if the exponential map $\exp : \mathfrak{g} \rightarrow G$ is a global diffeomorphism.

Let $f_{1}, f_{2}$ be two real valued functions on $G$. We say that $f_{1}$ and $f_{2}$ have the same behavior, that we write $f_{1} \asymp f_{2}$, when there exist $0<c \leq C$ such that for all $x \in G$, we have $c f_{1}(x) \leq f_{2}(x) \leq C f_{1}(x)$. 


\section{CHAPTER 1}

\section{Oscillatory integrals}

This chapter is the most technical part of this memoir. To outline its content, we shall first explain the situation on which the general theory is designed. Consider $\mathbb{B}$ a simply connected Lie group endowed with a left invariant Kählerian structure with negative curvature. Let also $\alpha$ be a strongly continuous and isometric action of $\mathbb{B}$ on a $C^{*}$-algebra $A$. In this context and from previous works of one of us, we have at our disposal two functions $\mathbf{m}_{\text {can }} \in C^{\infty}\left(\mathbb{B} \times \mathbb{B}, \mathbb{R}_{+}^{*}\right)$ and $S_{\text {can }} \in C^{\infty}(\mathbb{B} \times \mathbb{B}, \mathbb{R})$ such that setting for $\theta \in \mathbb{R}^{*}$

$$
K_{\theta}=\theta^{-\operatorname{dim} \mathbb{B}} \mathbf{m}_{\text {can }} \exp \left\{\frac{i}{\theta} S_{\text {can }}\right\} \in C^{\infty}(\mathbb{B} \times \mathbb{B}, \mathbb{C}),
$$

the following formula

$$
a \star_{\theta}^{\alpha} b:=\int_{\mathbb{B} \times \mathbb{B}} K_{\theta}(x, y) \alpha_{x}(a) \alpha_{y}(b) \mathrm{d} x \mathrm{~d} y, \quad a, b \in A,
$$

formally defines a one-parameter family of associative algebra structures on $A$. Since the function $\mathbf{m}_{\text {can }}$ is unbounded and since the map $\alpha(a):=\left[x \mapsto \alpha_{x}(a)\right]$ is constant in norm, the only hope to go beyond the formal level is to define the integral sign in (1.1) in an oscillatory way. What we are precisely looking for, is a pair $\left(A_{0}, \underline{\mathbf{D}}\right)$, where

- $A_{0}$ is a dense and $\alpha$-stable Fréchet subalgebra of $A$ with a topology (finer than the uniform one) determined by a countable set of semi-norms $\left\{\|\cdot\|_{j}\right\}_{j \in \mathbb{N}}$

- $\underline{\mathbf{D}}:=\left\{\mathbf{D}_{j}\right\}_{j \in \mathbb{N}}$ is a countable family of differential operators on $\mathbb{B} \times \mathbb{B}$, such that for all $a, b \in A_{0}$ and all $j \in \mathbb{N}$, the image under $\mathbf{D}_{j}$ of the map $[(x, y) \in$ $\left.\mathbb{B} \times \mathbb{B} \mapsto \mathbf{m}_{\text {can }}(x, y) \alpha_{x}(a) \alpha_{y}(b) \in A_{0}\right]$ belongs to $L^{1}\left(\mathbb{B} \times \mathbb{B},\left(A_{0},\|.\|_{j}\right)\right)$ and, denoting by $\mathbf{D}_{j}^{*}$ the formal adjoint of $\mathbf{D}_{j}$, such that

$$
\mathbf{D}_{j}^{*} \exp \left\{\frac{i}{\theta} S_{\text {can }}\right\}=\exp \left\{\frac{i}{\theta} S_{\text {can }}\right\} .
$$

Once such a pair is found, there is a clear way to give a meaning of $\star_{\theta}^{\alpha}$ on $A_{0}$, namely by the $\|\cdot\|_{j}$-absolutely convergent $A$-valued integral:

$$
a \star_{\theta}^{\alpha} b:=\theta^{-\operatorname{dim} \mathbb{B}} \int_{\mathbb{B} \times \mathbb{B}} \exp \left\{\frac{i}{\theta} S_{\text {can }}(x, y)\right\} \mathbf{D}_{j ; x, y}\left(\mathbf{m}_{\mathrm{can}}(x, y) \alpha_{x}(a) \alpha_{y}(b)\right) \mathrm{d} x \mathrm{~d} y .
$$

There is an obvious candidate for $A_{0}$, which is $A^{\infty}$, the Fréchet subalgebra of $A$ consisting of smooth vectors for the action $\alpha$ :

$$
A^{\infty}:=\left\{a \in A: \alpha(a)=\left[x \mapsto \alpha_{x}(a)\right] \in C^{\infty}(\mathbb{B}, A)\right\} .
$$

Recall that by the strong continuity assumption of the action, $A^{\infty}$ is dense in $A$. Then, the crucial observation, proved in Lemma 4.5, is that we have a continuous embedding:

$$
\alpha \otimes \alpha: A^{\infty} \times A^{\infty} \rightarrow \mathcal{B}^{\mu}\left(\mathbb{B} \times \mathbb{B}, A^{\infty}\right), \quad(a, b) \mapsto\left[(g, h) \mapsto \alpha_{g}(a) \alpha_{h}(b)\right],
$$


where the symbol space $\mathcal{B}^{\underline{\mu}}\left(\mathbb{B} \times \mathbb{B}, A^{\infty}\right)$ is defined in (1.9) and where $\underline{\mu}=\left\{\mu_{j}\right\}_{j \in \mathbb{N}}$ is a family of unbounded functions on $\mathbb{B} \times \mathbb{B}$, called weights (see Definition 1.1 below). We should already stress that it is because the group $\mathbb{B}$ is non Abelian (and noncompact) that we are forced to consider such symbol spaces associated to unbounded weights. Therefore, the problem becomes the construction of a family of differential operators $\underline{\mathbf{D}}=\left\{\mathbf{D}_{j}\right\}_{j \in \mathbb{N}}$, such that for all $j \in \mathbb{N}$, we have

$$
\mathbf{D}_{j}: \mathcal{B}^{\mu_{j}}\left(\mathbb{B} \times \mathbb{B}, A^{\infty}\right) \rightarrow L^{1}\left(\mathbb{B} \times \mathbb{B},\left(A^{\infty},\|\cdot\|_{j}\right)\right),
$$

and

$$
\mathbf{D}_{j}^{*} \exp \left\{\frac{i}{\theta} S_{\text {can }}\right\}=\exp \left\{\frac{i}{\theta} S_{\text {can }}\right\} \text {. }
$$

The construction of the family of differential operators $\underline{\mathbf{D}}$, and therefore of the associated notion of oscillatory integral, is rather involved and this is the whole subject of this chapter. Since in this memoir we eventually need an oscillatory integral for different groups (for $\mathbb{B} \times \mathbb{B}$ in chapters 3 and 4 and for $\mathbb{B}$ in chapter 7) and since we intend to apply the whole ideas of the present construction to situations involving other groups and other kernels, we have decided to formulate the results of this chapter in the greatest possible generality.

\subsection{Symbol spaces}

In this preliminary section, we consider a non-Abelian, weighted and Fréchet space valued version of the Laurent Schwartz space $\mathcal{B}$ of smooth functions that, together with all of their derivatives, are bounded. For reasons that will become clear later (cf. chapter 5), we refer to such function spaces as symbol spaces. They are constructed out of a family of specific functions on a Lie group $G$, that we call weights. The prototype of a weight for a non-Abelian Lie group is given in Definition 1.3. The key properties of these symbol spaces are established in Lemmas 1.8 and 1.12. In Lemma 1.15, we show on a fundamental example, how such spaces naturally appear in the context of non-Abelian Lie group actions.

Definition 1.1. Consider a connected real Lie group $G$ with Lie algebra $\mathfrak{g}$. An element $\mu \in C^{\infty}\left(G, \mathbb{R}_{+}^{*}\right)$ is called a weight if it satisfies the following properties:

(i) For every element $X \in \mathcal{U}(\mathfrak{g})$, there exist $C_{L}, C_{R}>0$ such that:

$$
|\widetilde{X} \mu| \leq C_{L} \mu \quad \text { and } \quad|\underline{X} \mu| \leq C_{R} \mu .
$$

(ii) There exist $C, L, R \in \mathbb{R}_{+}^{*}$ such that for all $g, h \in G$ we have:

$$
\mu(g h) \leq C \mu(g)^{L} \mu(h)^{R} .
$$

A pair $(L, R)$ as in item (ii) is called a sub-multiplicative degree of the weight $\mu$. A weight with sub-multiplicative degree $(1,1)$ is called a sub-multiplicative weight.

Remark 1.2. For $\mu \in C^{\infty}(G)$, we set $\mu^{\vee}(g):=\mu\left(g^{-1}\right)$. Then, from the relation $\widetilde{X} \mu^{\vee}=(\underline{X} \mu)^{\vee}$ for all $X \in \mathcal{U}(\mathfrak{g})$, we see that $\mu$ is a weight of sub-multiplicative degree $(L, R)$ if and only if $\mu^{\vee}$ is a weight of sub-multiplicative degree $(R, L)$. Moreover, a product of two weights is a weight and a (positive) power of a weight is a weight. Also, the tensor product of two weights is a weight on the direct product group. 
In the following, we construct a canonical and non-trivial weight for nonAbelian Lie groups. This specific weight is an important object as it will naturally and repeatedly appear in all our analysis (see for instance Lemmas 1.15, 1.42, 1.49, 4.3 and 4.5 .

Definition 1.3. Choosing an Euclidean norm $|$.$| on \mathfrak{g}$, for $x \in G$, we let $\left|\operatorname{Ad}_{x}\right|$ be the operator norm of the adjoint action of $G$ on $\mathfrak{g}$. The function

$$
\mathfrak{d}_{G}: G \rightarrow \mathbb{R}_{+}^{*}, \quad x \mapsto \sqrt{1+\left|\operatorname{Ad}_{x}\right|^{2}+\left|\operatorname{Ad}_{x^{-1}}\right|^{2}},
$$

is called the modular weight of $G$.

Lemma 1.4. The modular weight $\mathfrak{d}_{G}$ is a sub-multiplicative weight on $G$.

Proof. To prove that $\mathfrak{d}_{G}$ is a weight, we start from the relations for $X \in \mathfrak{g}$ and $x \in G$ :

$$
\begin{aligned}
\tilde{X}\left|\operatorname{Ad}_{x}\right|^{2} & =2 \sup _{Y \in \mathfrak{g},|Y|=1}\left\langle\operatorname{Ad}_{x} \circ \operatorname{ad}_{X}(Y), \operatorname{Ad}_{x}(Y)\right\rangle, \\
\tilde{X}\left|\operatorname{Ad}_{x^{-1}}\right|^{2} & =-2 \sup _{Y \in \mathfrak{g},|Y|=1}\left\langle\operatorname{ad}_{X} \circ \operatorname{Ad}_{x^{-1}}(Y), \operatorname{Ad}_{x^{-1}}(Y)\right\rangle, \\
\underline{X}\left|\operatorname{Ad}_{x}\right|^{2} & =-2 \sup _{Y \in \mathfrak{g},|Y|=1}\left\langle\operatorname{ad}_{X} \circ \operatorname{Ad}_{x}(Y), \operatorname{Ad}_{x}(Y)\right\rangle, \\
\underline{X}\left|\operatorname{Ad}_{x^{-1}}\right|^{2} & =2 \sup _{Y \in \mathfrak{g},|Y|=1}\left\langle\operatorname{Ad}_{x^{-1}} \circ \operatorname{ad}_{X}(Y), \operatorname{Ad}_{x^{-1}}(Y)\right\rangle .
\end{aligned}
$$

Then, we get by induction and for every $X \in \mathcal{U}(\mathfrak{g})$ of strictly positive homogeneous degree:

$$
\left|\widetilde{X} \mathfrak{d}_{G}(x)\right|,\left|\underline{X} \mathfrak{d}_{G}(x)\right| \leq\left|\operatorname{ad}_{X}\right| \frac{\left|\operatorname{Ad}_{x}\right|^{2}+\left|\operatorname{Ad}_{x^{-1}}\right|^{2}}{\sqrt{1+\left|\operatorname{Ad}_{x}\right|^{2}+\left|\operatorname{Ad}_{x^{-1}}\right|^{2}}} \leq\left|\operatorname{ad}_{X}\right| \mathfrak{d}_{G}(x),
$$

where, for $X \in \mathcal{U}(\mathfrak{g})$, we denote by $\left|\operatorname{ad}_{X}\right|$ the operator norm of the adjoint action of $\mathcal{U}(\mathfrak{g})$ on $\mathfrak{g}$. This implies condition (i) of Definition 1.1. Last, the inequality

$$
\left|\operatorname{Ad}_{g h}\right|=\left|\operatorname{Ad}_{g} \circ \operatorname{Ad}_{h}\right| \leq\left|\operatorname{Ad}_{g}\right|\left|\operatorname{Ad}_{h}\right|, \quad g, h \in G,
$$

implies the sub-multiplicativity of $\mathfrak{d}_{G}$.

We next give further informations regarding the modular weight of a semi-direct product of groups. These results will be used in Lemmas 2.27, 2.31, Proposition 4.20 and Lemma 7.18 .

Lemma 1.5. Let $G_{j}, j=1,2$, be two connected Lie groups with Lie algebras $\mathfrak{g}_{j}$, let $\mathbf{R}: G_{1} \rightarrow \operatorname{Aut}\left(G_{2}\right)$ be an extension homomorphism and consider the associated semi-direct product $G:=G_{1} \ltimes_{\mathbf{R}} G_{2}$ whose Lie algebra is denoted by $\mathfrak{g}=\mathfrak{g}_{1} \ltimes \mathfrak{g}_{2}$. Consider the adjoint actions $\mathrm{Ad}^{j}: G_{j} \rightarrow G L\left(\mathfrak{g}_{j}\right)$ and $\mathrm{Ad}: G \rightarrow G L(\mathfrak{g})$ and define the mapping

$$
\Phi: G_{2} \rightarrow \operatorname{Hom}\left(\mathfrak{g}_{1}, \mathfrak{g}_{2}\right), \quad g_{2} \mapsto\left[X_{1} \mapsto \operatorname{Ad}_{g_{2}}\left(X_{1}\right)-X_{1}\right] .
$$

Then, in the parametrization $G_{2} \times G_{1} \rightarrow G,\left(g_{2}, g_{1}\right) \mapsto g_{1} g_{2}$, we have the following behavior of the modular weight of the semi-direct product:

$$
\begin{aligned}
\mathfrak{d}_{G} \asymp\left[\left(g_{2}, g_{1}\right) \mapsto \mathfrak{d}_{G_{1}}\left(g_{1}\right)+(1\right. & +\left|\Phi\left(g_{2}\right) \circ \operatorname{Ad}_{g_{1}}^{1}\right|^{2}+\left|\operatorname{Ad}_{g_{2}}^{2} \circ \mathbf{R}_{g_{1}}\right|^{2} \\
& \left.\left.+\left|\mathbf{R}_{g_{1}^{-1}} \circ \Phi\left(g_{2}^{-1}\right)\right|^{2}+\left|\mathbf{R}_{g_{1}^{-1}} \circ \mathbf{A d}_{g_{2}^{-1}}^{2}\right|^{2}\right)^{1 / 2}\right],
\end{aligned}
$$


where we use the same notation for the extension homomorphism and its derivative:

$$
\mathfrak{g}_{2} \rightarrow \mathfrak{g}_{2},\left.\quad X \mapsto \frac{d}{d t}\right|_{t=0} \mathbf{R}_{g_{1}}\left(e^{t X}\right), \quad g_{1} \in G_{1} .
$$

In particular, we get the following behaviors of the restrictions of $\mathfrak{d}_{G}$ to the subgroups $G_{1}$ and $G_{2}$ :

$$
\begin{aligned}
& \left.\mathfrak{d}_{G}\right|_{G_{1}} \asymp \mathfrak{d}_{G_{1}}+\left[g_{1} \mapsto\left(1+\left|\mathbf{R}_{g_{1}}\right|^{2}+\left|\mathbf{R}_{g_{1}^{-1}}\right|^{2}\right)^{1 / 2}\right] \\
& \left.\mathfrak{d}_{G}\right|_{G_{2}} \asymp \mathfrak{d}_{G_{2}}+\left[g_{2} \mapsto\left(1+\left|\Phi\left(g_{2}\right)\right|^{2}+\left|\Phi\left(g_{2}^{-1}\right)\right|^{2}\right)^{1 / 2}\right] .
\end{aligned}
$$

Moreover, in the case of a direct product, we have the behavior

$$
\mathfrak{d}_{G_{1} \times G_{2}} \asymp \mathfrak{d}_{G_{1}} \otimes 1+1 \otimes \mathfrak{d}_{G_{2}} .
$$

Proof. Fix Euclidean structures on $\mathfrak{g}_{1}$ and on $\mathfrak{g}_{2}$ and induce one on $\mathfrak{g}_{1} \oplus \mathfrak{g}_{2}$ by declaring that $\mathfrak{g}_{1}$ is orthogonal to $\mathfrak{g}_{2}$. A direct computation shows that in the vector decomposition $\mathfrak{g}=\mathfrak{g}_{1} \oplus \mathfrak{g}_{2}$, the operator $\operatorname{Ad}_{g_{2} g_{1}}$ takes the following matrix form:

$$
\operatorname{Ad}_{g_{2} g_{1}}=\left(\begin{array}{cc}
\operatorname{Ad}_{g_{1}}^{1} & 0 \\
\Phi\left(g_{2}\right) \circ \operatorname{Ad}_{g_{1}}^{1} & \operatorname{Ad}_{g_{2}}^{2} \circ \mathbf{R}_{g_{1}}
\end{array}\right)
$$

with inverse given by

$$
\operatorname{Ad}_{\left(g_{2} g_{1}\right)^{-1}}=\left(\begin{array}{cc}
\operatorname{Ad}_{g_{1}^{-1}}^{1} & 0 \\
\mathbf{R}_{g_{1}^{-1}} \circ \Phi\left(g_{2}^{-1}\right) & \mathbf{R}_{g_{1}^{-1}} \circ \mathrm{Ad}_{g_{2}^{-1}}^{2}
\end{array}\right) .
$$

But on the finite dimensional vector space $\operatorname{End}(\mathfrak{g})$, the operator norm of $\operatorname{Ad}_{g_{2} g_{1}}$ is equivalent to its Hilbert-Schmidt norm, and the latter reads

$$
\left(\left|\operatorname{Ad}_{g_{1}}^{1}\right|^{2}+\left|\Phi\left(g_{2}\right) \circ \operatorname{Ad}_{g_{1}}^{1}\right|^{2}+\left|\operatorname{Ad}_{g_{2}}^{2} \circ \mathbf{R}_{g_{1}}\right|^{2}\right)^{1 / 2} .
$$

Similarly, the operator norm of $\operatorname{Ad}_{\left(g_{2} g_{1}\right)^{-1}}$ is equivalent to

$$
\left(\left|\operatorname{Ad}_{g_{1}^{-1}}^{1}\right|^{2}+\left|\mathbf{R}_{g_{1}^{-1}} \circ \Phi\left(g_{2}^{-1}\right)\right|+\left|\mathbf{R}_{g_{1}^{-1}} \circ \operatorname{Ad}_{g_{2}^{-1}}^{2}\right|^{2}\right)^{1 / 2},
$$

proving the first claim. The remaining statements follow immediately.

We should mention that the modular function, $\Delta_{G}$, is also a sub-multiplicative weight on $G$. Indeed the multiplicativity property implies that for every $X \in \mathcal{U}(\mathfrak{g})$ and $x \in G$ :

$$
\left(\widetilde{X} \Delta_{G}\right)(x)=\left(\widetilde{X} \Delta_{G}\right)(e) \Delta_{G}(x), \quad\left(\underline{X} \Delta_{G}\right)(x)=\left(\underline{X} \Delta_{G}\right)(e) \Delta_{G}(x) .
$$

However, this weight will not be of much interest in what follows.

The next notion will play a key role to establish density results for our symbol spaces. We assume from now on the Lie group $G$ to be non-compact.

Definition 1.6. Given two weights $\mu$ and $\hat{\mu}$, we say that $\hat{\mu}$ dominates $\mu$, which we denote by $\mu \prec \hat{\mu}$, if

$$
\lim _{g \rightarrow \infty} \frac{\mu(g)}{\hat{\mu}(g)}=0 .
$$

REMARK 1.7. For negatively curved Kählerian Lie groups, the modular weight has the crucial property to dominate the constant weight 1 (see Corollary 2.28 and Lemma 2.31. 
We now let $\mathcal{E}$ be a complex Fréchet space with topology underlying a countable family of semi-norms $\left\{\|\cdot\|_{j}\right\}_{j \in \mathbb{N}}$. Given a weight $\mu$, we first consider the following space of $\mathcal{E}$-valued functions on $G$ :

$$
\begin{aligned}
& \mathcal{B}^{\mu}(G, \mathcal{E}):= \\
& \quad\left\{F \in C^{\infty}(G, \mathcal{E}): \forall X \in \mathcal{U}(\mathfrak{g}), \forall j \in \mathbb{N}, \exists C>0:\|\widetilde{X} F\|_{j} \leq C \mu\right\} .
\end{aligned}
$$

When $\mathcal{E}=\mathbb{C}$ (respectively when $\mu=1$, respectively when $\mathcal{E}=\mathbb{C}$ and $\mu=1$ ), we denote $\mathcal{B}^{\mu}(G, \mathcal{E})$ by $\mathcal{B}^{\mu}(G)$ (respectively by $\mathcal{B}(G, \mathcal{E})$, respectively by $\mathcal{B}(G)$ ). We endow the space $\mathcal{B}^{\mu}(G, \mathcal{E})$ with the natural topology associated to the following semi-norms:

$$
\|F\|_{j, k, \mu}:=\sup _{X \in \mathcal{U}_{k}(\mathfrak{g})} \sup _{g \in G}\left\{\frac{\|\widetilde{X} F(g)\|_{j}}{\mu(g)|X|_{k}}\right\}, \quad j, k \in \mathbb{N},
$$

where $\mathcal{U}(\mathfrak{g})=\cup_{k \in \mathbb{N}} \mathcal{U}_{k}(\mathfrak{g})$ is the filtration described in (0.5) and $|.|_{k}$ is the norm on $\mathcal{U}_{k}(\mathfrak{g})$ defined in (0.7). Note that for $X=\sum_{|\beta| \leq k} C_{\beta} X^{\beta} \in \mathcal{U}_{k}(\mathfrak{g})$, we have

$$
\frac{\|\widetilde{X} F(g)\|_{j}}{|X|_{k}} \leq \frac{\sum_{|\beta| \leq k}\left|C_{\beta}\right|\left\|\widetilde{X^{\beta}} F(g)\right\|_{j}}{\sum_{|\beta| \leq k}\left|C_{\beta}\right|} \leq \max _{|\beta| \leq k}\left\|\widetilde{X^{\beta}} F(g)\right\|_{j},
$$

and hence

$$
\|F\|_{j, k, \mu} \leq \max _{|\beta| \leq k} \sup _{g \in G} \frac{\left\|\widetilde{X^{\beta}} F(g)\right\|_{j}}{\mu(g)}=\max _{|\beta| \leq k}\left\|\widetilde{X^{\beta}} F\right\|_{j, 0, \mu},
$$

which shows that the semi-norms (1.3) are well defined on $\mathcal{B}^{\mu}(G, \mathcal{E})$. When $\mathcal{E}=\mathbb{C}$ (respectively when $\mu=1$, respectively when $\mathcal{E}=\mathbb{C}$ and $\mu=1$ ), we denote the semi-norms (1.4) by $\|\cdot\|_{k, \mu}, k \in \mathbb{N}$ (respectively by $\|\cdot\|_{j, k}, j, k \in \mathbb{N}$, respectively by $\left.\|\cdot\|_{k}, k \in \mathbb{N}\right)$.

The basic properties of the spaces $\mathcal{B}^{\mu}(G, \mathcal{E})$ are established in the next lemma. They are essentially standard but are used all over the text. In particular, in the last item, we prove that $\mathcal{D}(G, \mathcal{E})$ is a dense subset of $\mathcal{B}^{\mu}(G, \mathcal{E})$ for the induced topology of $\mathcal{B}^{\hat{\mu}}(G, \mathcal{E})$, for $\hat{\mu}$ an arbitrary weight which dominates $\mu$. This fact will be used in a crucial way for the construction of the oscillatory integral given in Definition 1.31.

Let $C_{b}(G, \mathcal{E})$ be the Fréchet space of $\mathcal{E}$-valued continuous and bounded functions on $G$. The topology we consider on the latter is the one associated to the seminorms $\|F\|_{j}:=\sup _{g \in G}\|F(g)\|_{j}, j \in \mathbb{N}$. This space carries an action of $G$ by right-translations. This action is of course isometric but not necessarily strongly continuous. Consider therefore its closed subspace $C_{r u}(G, \mathcal{E})$ constituted by the right-uniformly continuous functions.

Lemma 1.8. Let $(G, \mathcal{E})$ as above and let $\mu, \nu$ and $\hat{\mu}$ be three weights on $G$.

(i) The right regular action $R^{\star}$ of $G$ on $C_{r u}(G, \mathcal{E})$ is isometric and strongly continuous.

(ii) Let $C_{r u}(G, \mathcal{E})^{\infty}$ be the subspace of $C_{r u}(G, \mathcal{E})$ of smooth vectors for the right regular action. Then $C_{r u}(G, \mathcal{E})^{\infty}$ identifies with $\mathcal{B}(G, \mathcal{E})$ as topological vector spaces. In particular, $\mathcal{B}(G, \mathcal{E})$ is Fréchet.

(iii) The left regular action $L^{\star}$ of $G$ on $\mathcal{B}(G, \mathcal{E})$ is isometric. 
(iv) The bilinear map:

$\mathcal{B}^{\mu}(G) \times \mathcal{B}^{\nu}(G, \mathcal{E}) \rightarrow \mathcal{B}^{\mu \nu}(G, \mathcal{E}), \quad(u, F) \mapsto[g \in G \mapsto u(g) F(g) \in \mathcal{E}]$ is continuous.

(v) The map

$$
\mathcal{B}^{\mu}(G, \mathcal{E}) \rightarrow \mathcal{B}(G, \mathcal{E}), \quad F \mapsto \mu^{-1} F,
$$

is an homeomorphism. In particular, the space $\mathcal{B}^{\mu}(G, \mathcal{E})$ is Fréchet as well.

(vi) For every $X \in \mathcal{U}(\mathfrak{g})$, the associated left invariant differential operator $\widetilde{X}$ acts continuously on $\mathcal{B}^{\mu}(G, \mathcal{E})$.

(vii) If there exists $C>0$ such that $\mu \leq C \hat{\mu}$, then $\mathcal{B}^{\mu}(G, \mathcal{E}) \subset \mathcal{B}^{\hat{\mu}}(G, \mathcal{E})$, continuously.

(viii) Assume that $\mu \prec \hat{\mu}$. Then the closure of $\mathcal{D}(G, \mathcal{E})$ in $\mathcal{B}^{\hat{\mu}}(G, \mathcal{E})$ contains $\mathcal{B}^{\mu}(G, \mathcal{E})$. In particular, the space $\mathcal{D}(G, \mathcal{E})$ is a dense subset of $\mathcal{B}^{\mu}(G, \mathcal{E})$ for the induced topology of $\mathcal{B}^{\hat{\mu}}(G, \mathcal{E})$.

Proof. (i) Recall that $G$ being locally compact and countable at infinity, the space $C_{b}(G, \mathcal{E})$ is Fréchet (by the same argument as in the proof of $\mathbf{3 2}$, Proposition 44.1 and Corollary 1]). The subspace $C_{r u}(G, \mathcal{E})$ is then closed as a uniform limit of right-uniformly continuous functions is right-uniformly continuous. Thus $C_{r u}(G, \mathcal{E})$ endowed with the induced topology is a Fréchet space as well.

Being isometric on $C_{b}(G, \mathcal{E})$, the right action is consequently isometric on $C_{r u}(G, \mathcal{E})$ too. Moreover, for any converging sequence $\left\{g_{n}\right\} \subset G$, with limit $g \in G$, and any $F \in C_{r u}(G, \mathcal{E})$, we have $\left\|\left(R_{g_{n}}^{\star}-R_{g}^{\star}\right) F\right\|_{j}=\sup _{g_{0} \in G}\left\|F\left(g_{0} g_{n}\right)-F\left(g_{0} g\right)\right\|_{j}$ which tends to zero due to the right-uniform continuity of $F$. Hence the right regular action $R^{\star}$ is strongly continuous on $C_{r u}(G, \mathcal{E})$.

(ii) Note that an element $F \in C_{r u}(G, \mathcal{E})^{\infty}$ is such that the map $\left[g \mapsto R_{g}^{\star} F\right]$ is smooth as a $C_{r u}(G, \mathcal{E})$-valued function on $G$. In particular, for every $X \in \mathcal{U}(\mathfrak{g}), \widetilde{X} F$ is bounded and smooth. This clearly gives the inclusion $C_{r u}(G, \mathcal{E})^{\infty} \subset \mathcal{B}(G, \mathcal{E})$. Reciprocally, $G$ acts on $\mathcal{B}(G, \mathcal{E})$ via the right regular representation. Indeed, for all $g \in G$ and $X \in \mathcal{U}(\mathfrak{g})$, we have

$$
\widetilde{X} \circ R_{g}^{\star}=R_{g}^{\star} \circ\left(\widetilde{\operatorname{Ad}_{g^{-1}} X}\right),
$$

and hence for $j, k \in \mathbb{N}$ and $F \in \mathcal{B}(G, \mathcal{E})$, we deduce

$$
\begin{aligned}
\left\|R_{g}^{\star} F\right\|_{j, k} & =\sup _{X \in \mathcal{U}_{k}(\mathfrak{g})} \sup _{g^{\prime} \in G}\left\{\frac{\left\|\left(\widehat{\mathrm{Ad}_{g^{-1}} X}\right) F\left(g^{\prime} g\right)\right\|_{j}}{|X|_{k}}\right\} \\
& =\sup _{X \in \mathcal{U}_{k}(\mathfrak{g})} \sup _{g^{\prime} \in G}\left\{\frac{\left\|\left(\widehat{\mathrm{Ad}_{g^{-1}} X}\right) F\left(g^{\prime}\right)\right\|_{j}}{|X|_{k}}\right\} \leq\left|\mathrm{Ad}_{g^{-1}}\right|_{k}\|F\|_{j, k},
\end{aligned}
$$

where $\left|\operatorname{Ad}_{g}\right|_{k}$ denotes the operator norm of the adjoint action of $G$ on the (finite dimensional) Banach space $\left(\mathcal{U}_{k}(\mathfrak{g}),|\cdot|_{k}\right)$. Now we have the inclusion $\mathcal{B}(G, \mathcal{E}) \subset$ $C_{r u}(G, \mathcal{E})$. Indeed, for $F \in \mathcal{B}(G), g \in G$ and for fixed $X \in \mathfrak{g}$, one observes that

$$
\begin{aligned}
|F(g \exp (t X))-F(g)| & =\left|\int_{0}^{t} \frac{\mathrm{d}}{\mathrm{d} \tau}(F(g \exp (\tau X))) \mathrm{d} \tau\right| \\
& =\left|\int_{0}^{t} \tilde{X} F(g \exp (\tau X)) \mathrm{d} \tau\right| \leq|X|_{1}\|F\|_{1}|t|,
\end{aligned}
$$


where $\|F\|_{1}=\sup _{X \in \mathcal{U}_{1}(\mathfrak{g})} \sup _{g \in G}|\widetilde{X} F| /|X|_{1}$. Hence, we obtain the right-uniform continuity of $F$. To show that $F \in \mathcal{B}(G)$ is a differentiable vector for the rightaction, we observe that

$$
\begin{aligned}
\left|\frac{1}{t}(F(g \exp (t X))-F(g))-(\widetilde{X} F)(g)\right| & \leq \int_{0}^{1}|(\widetilde{X} F)(g \exp (t \tau X))-(\widetilde{X} F)(g)| \mathrm{d} \tau \\
& \leq \int_{0}^{1} \int_{0}^{t \tau}\left|\left(\widetilde{X}^{2} F\right)\left(g \exp \left(\tau^{\prime} X\right)\right)\right| \mathrm{d} \tau^{\prime} \mathrm{d} \tau \\
& \leq|t| \sup _{g \in G}\left\{\left|\widetilde{X}^{2} F\right|(g)\right\} \leq\left|X^{2}\right|_{2}\|F\|_{2}|t|
\end{aligned}
$$

which tends to zero together with $t$. (Here $\|F\|_{2}=\sup _{X \in \mathcal{U}_{2}(\mathfrak{g})} \sup _{g \in G}|\widetilde{X} F| /|X|_{2}$.) This yields differentiability at the unit element. One gets it everywhere else by observing that

$$
\widetilde{X}_{g}\left(R_{g}^{\star} F\right)=R_{g}^{\star}(\widetilde{X} F), \quad \forall X \in \mathcal{U}(\mathfrak{g}), \quad \forall g \in G, \quad \forall F \in \mathcal{B}(G) .
$$

An induction on the order of derivation implies $\mathcal{B}(G) \subset C_{r u}(G)^{\infty}$. The $\mathcal{E}$-valued case is entirely similar.

The assertion concerning the topology follows from the definition of the topology on smooth vectors $\mathbf{3 5}$.

(iii) The fact that $G$ acts isometrically on $\mathcal{B}(G, \mathcal{E})$ via the left regular representation, follows from

$$
\begin{aligned}
\left\|L_{g}^{\star} F\right\|_{j, k} & =\sup _{X \in \mathcal{U}_{k}(\mathfrak{g})} \sup _{g^{\prime} \in G} \frac{\left\|\widetilde{X}\left(L_{g}^{\star} F\right)\left(g^{\prime}\right)\right\|_{j}}{|X|_{k}}=\sup _{X \in \mathcal{U}_{k}(\mathfrak{g})} \sup _{g^{\prime} \in G} \frac{\left\|\left(L_{g}^{\star} \widetilde{X} F\right)\left(g^{\prime}\right)\right\|_{j}}{|X|_{k}} \\
& =\sup _{X \in \mathcal{U}_{k}(\mathfrak{g})} \sup _{g^{\prime} \in G} \frac{\left\|\widetilde{X} F\left(g^{-1} g^{\prime}\right)\right\|_{j}}{|X|_{k}}=\sup _{X \in \mathcal{U}_{k}(\mathfrak{g})} \sup _{g^{\prime} \in G} \frac{\left\|\widetilde{X} F\left(g^{\prime}\right)\right\|_{j}}{|X|_{k}}=\|F\|_{j, k} .
\end{aligned}
$$

(iv) Let $u \in \mathcal{B}^{\mu}(G)$ and $F \in \mathcal{B}^{\nu}(G, \mathcal{E})$. Using Sweedler's notation (0.2), we have for $j, k \in \mathbb{N}$ :

$$
\begin{aligned}
\|u F\|_{j, k, \mu \nu} & =\sup _{X \in \mathcal{U}_{k}(\mathfrak{g})} \sup _{g \in G} \frac{\|\widetilde{X}(u F)(g)\|_{j}}{\mu(g) \nu(g)|X|_{k}} \\
& \leq \sup _{X \in \mathcal{U}_{k}(\mathfrak{g})} \sup _{g \in G} \sum_{(X)} \frac{\left|\left(\widetilde{X}_{(1)} u\right)(g)\right|\left\|\left(\widetilde{X}_{(2)} F\right)(g)\right\|_{j}}{\mu(g) \nu(g)|X|_{k}} \\
& \leq\left(\sup _{X \in \mathcal{U}_{k}(\mathfrak{g})} \sum_{(X)} \frac{\left.\left|X_{(1)}\right|\right|_{k}\left|X_{(2)}\right|_{k}}{|X|_{k}}\right)\|u\|_{k, \mu}\|F\|_{j, k, \nu} .
\end{aligned}
$$

Now, for $X=\sum_{|\beta| \leq k} C_{\beta} X^{\beta} \in \mathcal{U}_{k}(\mathfrak{g})$, expanded in the PBW basis (0.4), we have

$$
\Delta_{\mathcal{U}}(X)=\sum_{|\beta| \leq k} C_{\beta} \sum_{\gamma \leq \beta}\left(\begin{array}{l}
\beta \\
\gamma
\end{array}\right) X^{\gamma} \otimes X^{\beta-\gamma}
$$

which, with $m$ the dimension of $\mathfrak{g}$, implies that

$$
\left.\sum_{(X)}\left|X_{(1)}\right|\right|_{k}\left|X_{(2)}\right|_{k} \leq \sum_{|\beta| \leq k}\left|C_{\beta}\right| \sum_{\gamma \leq \beta}\left(\begin{array}{l}
\beta \\
\gamma
\end{array}\right) \leq 2^{m k} \sum_{|\beta| \leq k}\left|C_{\beta}\right|=2^{m k}|X|_{k}
$$

Hence we get

$$
\|u F\|_{j, k, \mu \nu} \leq 2^{m k}\|u\|_{k, \mu}\|F\|_{j, k, \nu}
$$


proving the continuity.

(v) Note first that since $\mu$ is a weight, for every $X \in \mathcal{U}(\mathfrak{g})$ there is a constant $C>0$ such that $\left|\tilde{X}\left(\mu^{-1}\right)\right| \leq C \mu^{-1}$. This easily follows by induction once one has noticed that for $X \in \mathfrak{g}$, we have $\widetilde{X}\left(\mu^{-1}\right)=-(\widetilde{X} \mu) \mu^{-2}$. This entails that if $\mu \in \mathcal{B}^{\mu}(G)$ then $\mu^{-1} \in \mathcal{B}^{\mu^{-1}}(G)$, even if $\mu^{-1}$ may not be a weight in the sense of Definition [1.1, as it may not have any sub-multiplicative degree. Then, (v) follows from (iv), where the existence of a sub-multiplicative degree is not used in that proof.

(vi) This assertion follows immediately from equation (1.4) and (vii) follows easily from equation (1.3).

(viii) Choose an increasing sequence $\left\{C_{n}\right\}_{n \in \mathbb{N}}$ of relatively compact open subsets in $G$, such that $\lim _{n} C_{n}=G$. Pick $0 \leq \psi \in \mathcal{D}(G)$ of $L^{1}(G)$-norm one and define

$$
e_{n}:=\int_{G} \psi(g) R_{g}^{\star}\left(\chi_{n}\right) \mathrm{d}_{G}(g),
$$

where $\chi_{n}$ denotes the characteristic function of $C_{n}$. It is clear that $e_{n}$ is an increasing family of smooth compactly supported functions, which by Lebesgue dominated convergence, converges point-wise to the unit function. Moreover, for all $F \in \mathcal{B}^{\mu}(G, \mathcal{E})$ and $\hat{\mu}$ a weight dominating $\mu$, we have

$$
\begin{aligned}
\left\|\left(1-e_{n}\right) F\right\|_{j, 0, \hat{\mu}} & =\sup _{g \in G}\left\{\frac{1}{\hat{\mu}(g)}\left(1-e_{n}(g)\right)\|F(g)\|_{j}\right\} \\
& \leq\|F\|_{j, 0, \mu} \sup _{g \in G}\left\{\frac{\mu(g)}{\hat{\mu}(g)}\left(1-e_{n}(g)\right)\right\},
\end{aligned}
$$

which converges to zero when $n$ goes to infinity, since $\mu / \hat{\mu} \rightarrow 0$ when $g \rightarrow \infty$ and for fixed $g \in G, 1-e_{n}(g)$ decreases to zero when $n \rightarrow \infty$. We need to show that the same property holds true for all the semi-norms $\|\cdot\|_{j, k, \hat{\mu}}, k \geq 1$. We use an induction. First note that if $X \in \mathfrak{g}$, then we have

$$
\begin{aligned}
\tilde{X} e_{n}=\left.\frac{d}{d t}\right|_{t=0} R_{e^{t X}}^{\star}\left(e_{n}\right) & =\left.\frac{d}{d t}\right|_{t=0} \int_{G} \psi(g) R_{e^{t X}}^{\star} R_{g}^{\star}\left(\chi_{n}\right) \mathrm{d}_{G}(g) \\
& =\left.\frac{d}{d t}\right|_{t=0} \int_{G} \psi\left(e^{-t X} g\right) R_{g}^{\star}\left(\chi_{n}\right) \mathrm{d}_{G}(g) \\
& =\int_{G}(\underline{X} \psi)(g) R_{g}^{\star}\left(\chi_{n}\right) \mathrm{d}_{G}(g) .
\end{aligned}
$$

A routine inductive argument then gives

$$
\widetilde{X} e_{n}=\int_{G}(\underline{X} \psi)(g) R_{g}^{\star}\left(\chi_{n}\right) \mathrm{d}_{G}(g), \quad \forall X \in \mathcal{U}(\mathfrak{g}),
$$

which entails

$$
\left\|\widetilde{X} e_{n}\right\|_{\infty} \leq\|\underline{X} \psi\|_{1}<\infty, \quad \forall X \in \mathcal{U}(\mathfrak{g}) .
$$

(This means that the sequence $\left\{e_{n}\right\}_{n \in \mathbb{N}}$ belongs to $\mathcal{B}(G)$, uniformly in $n$.) Now, assume that $\left\|\left(1-e_{n}\right) F\right\|_{j, k, \hat{\mu}} \rightarrow 0, n \rightarrow \infty$, for a given $k \in \mathbb{N}$, for all $F \in \mathcal{B}^{\mu}(G, \mathcal{E})$ and all $j \in \mathbb{N}$. From the same reasoning as those leading to (1.4) and with $X^{\beta}$ the element of the PBW basis of $\mathcal{U}(\mathfrak{g})$ defined in (0.4), we see that

$$
\left\|\left(1-e_{n}\right) F\right\|_{j, k+1, \hat{\mu}} \leq\left\|\left(1-e_{n}\right) F\right\|_{j, k, \hat{\mu}}+\max _{|\beta|=k+1}\left\|\widetilde{X^{\beta}}\left(\left(1-e_{n}\right) F\right)\right\|_{j, 0, \hat{\mu}} .
$$


We only need to show that the second term in the inequality above goes to zero when $n \rightarrow \infty$, as the first does by induction hypothesis. Writing $X^{\beta}=X^{\gamma} X$, with $|\gamma|=k$ and $X \in \mathfrak{g}$, by virtue of the Leibniz rule, we get

$$
\widetilde{X^{\gamma}} \widetilde{X}\left(\left(1-e_{n}\right) F\right)=-\widetilde{X^{\gamma}}\left(\left(\widetilde{X} e_{n}\right) F\right)+\widetilde{X^{\gamma}}\left(\left(1-e_{n}\right) \widetilde{X} F\right) .
$$

Note that

$$
\left\|\widetilde{X^{\gamma}}\left(\left(1-e_{n}\right) \widetilde{X} F\right)\right\|_{j, 0, \hat{\mu}} \leq\left\|\left(1-e_{n}\right) \widetilde{X} F\right\|_{j, k, \hat{\mu}},
$$

which converges to zero when $n \rightarrow \infty$ by induction hypothesis, since $\widetilde{X} F \in \mathcal{B}^{\mu}(G, \mathcal{E})$ and $|\gamma|=k$. Regarding the first term, we have using Sweedler's notations (0.2) and for a finite sum:

$$
\widetilde{X^{\gamma}}\left(\left(\widetilde{X} e_{n}\right) F\right)=\sum_{\left(X^{\gamma}\right)}\left(\widetilde{X_{(1)}^{\gamma}} \widetilde{X} e_{n}\right)\left(\widetilde{X_{(2)}^{\gamma}} F\right)
$$

Note that $\int_{G} \underline{P X} \psi \mathrm{d}_{G}=0$ for any $P \in \mathcal{U}(\mathfrak{g}), X \in \mathfrak{g}$ any $\psi \in \mathcal{D}(G)$. Indeed, this follows from an inductive argument starting with

$$
\int_{G} \underline{X} \psi(g) \mathrm{d}_{G}(g)=\left.\frac{d}{d t}\right|_{t=0} \int_{G} L_{e^{t X}}^{\star}(\psi)(g) \mathrm{d}_{G}(g)=\left.\frac{d}{d t}\right|_{t=0} \int_{G} \psi(g) \mathrm{d}_{G}(g)=0,
$$

for all $X \in \mathfrak{g}$. Using (1.8), we arrive at

$$
\left.\widetilde{X^{\gamma}}\left(\left(\widetilde{X} e_{n}\right) F\right)=\sum_{\left(X^{\gamma}\right)}\left(\int_{G}\left(\underline{X}^{\gamma}(1) \underline{X} \psi\right)(g)\left(R_{g}^{\star}\left(\chi_{n}\right)-1\right)\right) \mathrm{d}_{G}(g)\right) \widetilde{X}_{(2)} F,
$$

which converges to zero in the norms $\|\cdot\|_{j, 0, \hat{\mu}}, j \in \mathbb{N}$, since it is a finite sum of terms of the form $\left(1-e_{n}\right) F$ (with possibly re-defined $F$ 's in $\mathcal{B}^{\mu}(G, \mathcal{E})$ and $\psi$ 's in $\mathcal{D}(G))$.

REMark 1.9. We stress that as $\mu \prec \hat{\mu}$ implies that $\mu \leq C \hat{\mu}$ for some $C>0$ (since $G$ is locally compact), in Lemma 1.8, item (viii) strengthens item (vii). In particular, when $\mu \prec \hat{\mu}, \mathcal{B}^{\mu}(G, \mathcal{E})$ is continuously contained in $\mathcal{B}^{\hat{\mu}}(G, \mathcal{E})$. This fact is of upmost importance for the continuity of the oscillatory integral, given in Definition 1.31.

REMARK 1.10. On $\mathcal{B}(G, \mathcal{E})$, the left regular action is generally not strongly continuous and the right regular action is never isometric unless $G$ is Abelian.

We now generalize the spaces $\mathcal{B}^{\mu}(G, \mathcal{E})$, by allowing a certain behavior at infinity of the $\mathcal{E}$-valued functions on $G$, which is not necessarily uniform with respect to the semi-norm index. We first introduce some more notations.

Definition 1.11. Let $J$ be a countable set and let $\mu:=\left\{\mu_{j}\right\}_{j \in J}$ be an associated family of weights on a Lie group $G$. We denote by $(\underline{L}, \underline{R}):=\left\{\left(L_{j}, R_{j}\right)\right\}_{j \in J}$ the associated family of sub-multiplicative degrees. Given two families of weights $\mu, \hat{\mu}$, we say that $\underline{\hat{\mu}}$ dominates $\underline{\mu}$, denoted by $\underline{\mu} \prec \underline{\hat{\mu}}$, if $\hat{\mu}_{j}$ dominates $\mu_{j}$ for all $j \bar{\epsilon} \bar{J}$. The term by term product (respectively tensor product) of two families of weights $\underline{\mu}$ and $\underline{\nu}$, is denoted by $\underline{\mu} . \underline{\nu}$ (respectively by $\underline{\mu} \otimes \underline{\nu}$ ).

Consider a Fréchet space $\mathcal{E}$ and $\underline{\mu}$ a countable family of weights on $G$. We then define

$$
\begin{aligned}
& \mathcal{B}^{\underline{\mu}}(G, \mathcal{E}):= \\
& \quad\left\{F \in C^{\infty}(G, \mathcal{E}): \forall X \in \mathcal{U}(\mathfrak{g}), \forall j \in \mathbb{N}, \exists C>0:\|\widetilde{X} F\|_{j} \leq C \mu_{j}\right\} .
\end{aligned}
$$


We endow the latter space with the following set of the semi-norms:

$$
\|F\|_{j, k, \underline{\mu}}:=\sup _{X \in \mathcal{U}_{k}(\mathfrak{g})} \sup _{g \in G}\left\{\frac{\|\widetilde{X} F(g)\|_{j}}{\mu_{j}(g)|X|_{k}}\right\}, \quad j, k \in \mathbb{N},
$$

As expected, the space $\mathcal{B}^{\mu}(G, \mathcal{E})$ is Fréchet for the topology induced by the seminorms (1.10) and most of the properties of Lemma 1.8 remain true.

Lemma 1.12. Let $(G, \mathcal{E})$ as above and let $\underline{\mu}, \underline{\nu}$ and $\underline{\hat{\mu}}$ be three families of weights on $G$.

(i) The space $\mathcal{B}^{\underline{\mu}}(G, \mathcal{E})$ is Fréchet.

(ii) Let $(\underline{L}, \underline{R})$ be the sub-multiplicative degree of $\underline{\mu}$. Then, for every $g \in G$ the left-translation $L_{g}^{\star}$ defines a continuous map from $\mathcal{B} \underline{\mu}(G, \mathcal{E})$ to $\mathcal{B} \underline{\lambda}(G, \mathcal{E})$, where $\underline{\lambda}:=\left\{\lambda_{j}\right\}_{j \in \mathbb{N}}$ with $\lambda_{j}:=\mu_{j}^{R_{j}}$.

(iii) The bilinear map:

$$
\mathcal{B}^{\underline{\mu}}(G) \times \mathcal{B}^{\underline{\nu}}(G, \mathcal{E}) \rightarrow \mathcal{B}^{\underline{\mu}} \cdot \underline{\underline{\nu}}(G, \mathcal{E}), \quad(u, F) \mapsto[g \in G \mapsto u(g) F(g) \in \mathcal{E}],
$$

is continuous.

(iv) For every $X \in \mathcal{U}(\mathfrak{g})$, the left invariant differential operator $\widetilde{X}$ acts continuously on $\mathcal{B}^{\mu}(G, \mathcal{E})$.

(v) If for every $j \in \mathbb{N}$, there exists $C_{j}>0$ such that $\mu_{j} \leq C_{j} \hat{\mu}_{j}$, then $\mathcal{B}^{\underline{\mu}}(G, \mathcal{E}) \subset \mathcal{B}^{\hat{\mu}}(G, \mathcal{E})$.

(vi) Assume that $\mu \prec \hat{\mu}$. Then, the closure of $\mathcal{D}(G, \mathcal{E})$ in $\mathcal{B}-\hat{\mu}(G, \mathcal{E})$ contains $\mathcal{B}^{\mu}(G, \mathcal{E})$. In particular, $\mathcal{D}(G, \mathcal{E})$ is a dense subset of $\mathcal{B}^{\mu}(G, \mathcal{E})$ for the induced topology of $\mathcal{B} \hat{\underline{\mu}}(G, \mathcal{E})$.

Proof. (i) For each $j \in \mathbb{N}$, define $\|\cdot\|_{j}:=\sum_{k=0}^{j}\|\cdot\|_{k}$. Clearly, the topologies on $\mathcal{E}$ associated with the families of semi-norms $\left\{\|\cdot\|_{j}\right\}_{j \in \mathbb{N}}$ and $\left\{\|\cdot\|_{j}\right\}_{j \in \mathbb{N}}$ are equivalent. Thus, we may assume without loss of generality that the family of semi-norms $\left\{\|\cdot\|_{j}\right\}_{j \in \mathbb{N}}$ is increasing. We start by recalling the standard realization of the Fréchet space $\left(\mathcal{E},\left\{\|\cdot\|_{j}\right\}_{j \in \mathbb{N}}\right)$ as a projective limit. One considers the null spaces $V_{j}:=\left\{v \in \mathcal{E} \mid\|v\|_{j}=0\right\}$ and form the normed quotient spaces $\dot{\mathcal{E}}_{j}:=\mathcal{E} / V_{j}$. Denoting by $\mathcal{E}_{j}$ the Banach completion of the latter, the family of semi-norms being increasing, one gets, for every pair of indices $i \leq j$, a natural continuous linear mapping $g_{j i}: \mathcal{E}_{j} \rightarrow \mathcal{E}_{i}$. The Fréchet space $\mathcal{E}$ is then isomorphic to the subspace $\tilde{\mathcal{E}}$ of the product space $\prod_{j} \mathcal{E}_{j}$ constituted by the elements $(x) \in \prod_{j} \mathcal{E}_{j}$ such that $x_{i}=g_{j i}\left(x_{j}\right)$. Within this setting, the subspace $\tilde{\mathcal{E}}$ is endowed with the projective topology associated with the family of maps $\left\{f_{j}: \tilde{\mathcal{E}} \rightarrow \mathcal{E}_{j}:(x) \mapsto x_{j}\right\}$ (i.e. the coarsest topology that renders continuous each of the $f_{j}$ 's- see e.g. [29, pp. 50-52]). Within this context, we then observe that the topology on $\mathcal{B}^{\mu}(G, \mathcal{E}) \simeq \mathcal{B} \underline{\mu}(G, \tilde{\mathcal{E}})$ induced by the semi-norms (1.10) consists of the projective topology associated with the mappings $\phi_{j}: \mathcal{B}^{\mu}(G, \tilde{\mathcal{E}}) \rightarrow \mathcal{B}^{\mu_{j}}\left(G, \mathcal{E}_{j}\right): F \mapsto f_{j} \circ F$. Next we consider a Cauchy sequence $\left\{F_{n}\right\}_{n \in \mathbb{N}}$ in $\mathcal{B}^{\mu}(G, \tilde{\mathcal{E}})$. Since every space $\mathcal{B}^{\mu_{j}}\left(G, \mathcal{E}_{j}\right)$ is Fréchet, each sequence $\left\{f_{j} \circ F_{n}\right\}_{n \in \mathbb{N}}$ converges in $\mathcal{B}^{\mu_{j}}\left(G, \mathcal{E}_{j}\right)$ to an element denoted by $F^{j}$. Moreover, for every $g \in G$, one has

$$
\begin{aligned}
\left\|g_{j i} \circ F^{j}(g)-F^{i}(g)\right\|_{i} & =\left\|g_{j i} \circ F^{j}(g)-f_{i} \circ F_{n}(g)+f_{i} \circ F_{n}(g)-F^{i}(g)\right\|_{i} \\
& \leq\left\|g_{j i} \circ\left(F^{j}-f_{j} \circ F_{n}\right)(g)\right\|_{i}+\left\|f_{i} \circ F_{n}(g)-F^{i}(g)\right\|_{i}
\end{aligned}
$$


which can be rendered as small as we want since every $g_{j i}$ is continuous. Hence $g_{j i} \circ F^{j}=F^{i}$ which amounts to say that $\mathcal{B}^{\mu}(G, \tilde{\mathcal{E}})$ is complete.

(ii) Let $F \in \mathcal{B}^{\mu}(G, \mathcal{E})$ and $g \in G$ and set $\lambda_{j}:=\mu_{j}^{R_{j}}$. We have for $j, k \in \mathbb{N}$ :

$$
\begin{aligned}
\left\|L_{g}^{\star} F\right\|_{j, k, \underline{\lambda}} & =\sup _{X \in \mathcal{U}_{k}(\mathfrak{g})} \sup _{g^{\prime} \in G} \frac{\left\|\widetilde{X}\left(L_{g}^{\star} F\right)\left(g^{\prime}\right)\right\|_{j}}{\mu_{j}\left(g^{\prime}\right)^{R_{j}}|X|_{k}}=\sup _{X \in \mathcal{U}_{k}(\mathfrak{g})} \sup _{g^{\prime} \in G} \frac{\left\|\left(L_{g}^{\star} \widetilde{X} F\right)\left(g^{\prime}\right)\right\|_{j}}{\mu_{j}\left(g^{\prime}\right)^{R_{j}}|X|_{k}} \\
& =\sup _{X \in \mathcal{U}_{k}(\mathfrak{g})} \sup _{g^{\prime} \in G} \frac{\left\|(\widetilde{X} F)\left(g^{-1} g^{\prime}\right)\right\|_{j}}{\mu_{j}\left(g^{\prime}\right)^{R_{j}}|X|_{k}} \leq \mu_{j}\left(g^{-1}\right)^{L_{j}}\|F\|_{j, k, \underline{\mu}} .
\end{aligned}
$$

Items (iii), (iv), (v) and (vi) are proved in the same way as their counterparts in Lemma 1.8 .

REMARK 1.13. In the same fashion as in Remark 1.9 , we see that when $\underline{\mu} \prec \underline{\hat{\mu}}$, then $\mathcal{B} \underline{\underline{\mu}}(G, \mathcal{E}) \subset \mathcal{B}^{\hat{\mu}}(G, \mathcal{E})$ continuously.

In Lemma 1.15, we show on a first example, how the notion of $\mathcal{B}$-spaces for families of weights, naturally appears in the context of non-Abelian Lie group actions. We start by a preliminary result. We fix an Euclidean structure on $\mathfrak{g}$, such that the basis $\left\{X_{1}, \ldots, X_{n}\right\}$ (from which we have constructed the PBW basis (0.4)) is orthonormal.

Lemma 1.14. For $g \in G$ and $k \in \mathbb{N}$, denote by $\left|\operatorname{Ad}_{g}\right|_{k}$ the operator norm of the adjoint action $\operatorname{Ad}$ of $G$ on the finite dimensional Banach space $\left(\mathcal{U}_{k}(\mathfrak{g}),\left.|\cdot|\right|_{k}\right)$. Then, for each $k \in \mathbb{N}$, there exists a constant $C_{k}>0$, such that for all $g \in G$

$$
\left|\operatorname{Ad}_{g}\right|_{k} \leq C_{k} \mathfrak{d}_{G}(g)^{k} .
$$

where $\mathfrak{d}_{G} \in C^{\infty}(G)$ is the modular weight (given in Definition 1.3).

Proof. Note first that for all $k \in \mathbb{N}$, there exists a constant $\omega_{k}>0$ such that for all $X \in \mathcal{U}_{k_{1}}(\mathfrak{g})$ and $Y \in \mathcal{U}_{k_{2}}(\mathfrak{g})$, we have

$$
|X Y|_{k_{1}+k_{2}} \leq \omega_{k_{1}+k_{2}}|X|_{k_{1}}|Y|_{k_{2}} .
$$

Indeed, observe that if

$$
X=\sum_{|\beta| \leq k_{1}} C_{\beta}^{1} X^{\beta} \in \mathcal{U}_{k_{1}}(\mathfrak{g}) \text { and } Y=\sum_{|\beta| \leq k_{2}} C_{\beta}^{2} X^{\beta} \in \mathcal{U}_{k_{2}}(\mathfrak{g}),
$$

we have

$$
X Y=\sum_{\left|\beta_{1}\right| \leq k_{1},\left|\beta_{2}\right| \leq k_{2}} C_{\beta_{1}}^{1} C_{\beta_{2}}^{2} \sum_{|\beta| \leq\left|\beta_{1}+\beta_{2}\right|} \omega_{\beta}^{\beta_{1}, \beta_{2}} X^{\beta}
$$

where the constants $\omega_{\beta}^{\beta_{1}, \beta_{2}}$ are defined in (0.6). The sub-additivity of the norm $|\cdot|_{k_{1}+k_{2}}$ then entails that

$$
|X Y|_{k_{1}+k_{2}} \leq \sum_{\left|\beta_{1}\right| \leq k_{1},\left|\beta_{2}\right| \leq k_{2}}\left|C_{\beta_{1}}^{1}\right|\left|C_{\beta_{2}}^{2}\right| \sum_{|\beta| \leq\left|\beta_{1}+\beta_{2}\right|}\left|\omega_{\beta}^{\beta_{1}, \beta_{2}}\right| .
$$

Thus, it leads to defining

$$
\omega_{k_{1}+k_{2}}:=\sup _{\left|\beta_{1}+\beta_{2}\right| \leq k_{1}+k_{2}} \sum_{|\beta| \leq\left|\beta_{1}+\beta_{2}\right|}\left|\omega_{\beta}^{\beta_{1}, \beta_{2}}\right|,
$$

and the inequality (1.11) is proved. Next, for

$$
X=\sum_{|\beta| \leq k} C_{\beta} X_{1}^{\beta_{1}} \ldots X_{m}^{\beta_{m}} \in \mathcal{U}_{k}(\mathfrak{g})
$$


we have

$$
\operatorname{Ad}_{g}(X)=\sum_{|\beta| \leq k} C_{\beta}\left(\operatorname{Ad}_{g}\left(X_{1}\right)\right)^{\beta_{1}} \ldots\left(\operatorname{Ad}_{g}\left(X_{m}\right)\right)^{\beta_{m}} \in \mathcal{U}_{k}(\mathfrak{g})
$$

and thus by the previous considerations, we deduce

$$
\left|\operatorname{Ad}_{g}(X)\right|_{k} \leq \sum_{|\beta| \leq k}\left|C_{\beta}\right|\left(\prod_{j=2}^{|\beta|} \omega_{j}\right)\left|\operatorname{Ad}_{g}\left(X_{1}\right)\right|_{1}^{\beta_{1}}\left|\operatorname{Ad}_{g}\left(X_{2}\right)\right|_{1}^{\beta_{2}} \ldots\left|\operatorname{Ad}_{g}\left(X_{m}\right)\right|_{1}^{\beta_{m}}
$$

As the restriction of the norm $|.|_{1}$ from $\mathcal{U}_{1}(\mathfrak{g})$ to $\mathfrak{g}$ coincides with the $\ell^{1}$-norm of $\mathfrak{g}$ within the basis $\left\{X_{1}, \ldots, X_{m}\right\}$, we deduce for $j=1, \ldots, m$ and with $\left|\operatorname{Ad}_{g}\right|$ the operator norm of $\operatorname{Ad}_{g}$ with respect to the Euclidean structure of $\mathfrak{g}$ chosen:

$$
\left|\operatorname{Ad}_{g}\left(X_{j}\right)\right|_{1} \leq \sqrt{m}\left|\operatorname{Ad}_{g}\left(X_{j}\right)\right| \leq \sqrt{m}\left|\operatorname{Ad}_{g}\right|\left|X_{j}\right|_{\mathfrak{g}}=\sqrt{m}\left|\operatorname{Ad}_{g}\right|
$$

as $X_{j} \in \mathfrak{g}$ belongs to the unit sphere of $\mathfrak{g}$ for the Euclidean norm $|\cdot| \mathfrak{g}$. This implies

$$
\left|\operatorname{Ad}_{g}(X)\right|_{k} \leq m^{k / 2}\left(\sup _{|\beta| \leq k} \prod_{j=2}^{|\beta|} \omega_{j}\right)\left|\operatorname{Ad}_{g}\right|^{k},
$$

and the result follows from Definition 1.3 .

LEMMA 1.15. Let $\mu$ be a family of weights on $G$, with sub-multiplicative degree $(\underline{L}, \underline{R})$. Then the linear map

$$
\mathcal{R}:=\left[F \in C^{\infty}(G, \mathcal{E}) \mapsto\left[g \mapsto R_{g}^{\star} F\right] \in C^{\infty}\left(G, C^{\infty}(G, \mathcal{E})\right)\right],
$$

is continuous from $\mathcal{B} \underline{\mu}(G, \mathcal{E})$ to $\mathcal{B}^{\underline{\nu}}(G, \mathcal{B} \underline{\lambda}(G, \mathcal{E}))$, where $\underline{\nu}:=\left\{\nu_{j, k}\right\}_{j, k \in \mathbb{N}}$ and $\underline{\lambda}:=$ $\left\{\lambda_{j}\right\}_{j \in \mathbb{N}}$ with

$$
\nu_{j, k}:=\mu_{j}^{R_{j}} \mathfrak{d}_{G}^{k} \quad \text { and } \quad \lambda_{j}:=\mu_{j}^{L_{j}} .
$$

More precisely, labeling by $(j, k) \in \mathbb{N}^{2}$ the semi-norm $\|\cdot\|_{j, k, \underline{\lambda}}$ of $\mathcal{B} \underline{\lambda}(G, \mathcal{E})$, for each $\left(j, k, k^{\prime}\right) \in \mathbb{N}^{3}$, there exists a constant $C>0$, such that for all $F \in \mathcal{B} \underline{\mu}(G, \mathcal{E})$, we have

$$
\|\mathcal{R}(F)\|_{(j, k), k^{\prime}, \underline{\nu}} \leq C\|F\|_{j, k+k^{\prime}, \underline{\mu}} .
$$

Proof. Using the relation (1.5), we obtain for $X \in \mathcal{U}_{k^{\prime}}(\mathfrak{g}), F \in \mathcal{B} \underline{\mu}(G, \mathcal{E})$ and $g \in G$ :

$$
\left\|\widetilde{X}_{g} R_{g}^{\star}(F)\right\|_{j, k, \underline{\lambda}}=\left\|R_{g}^{\star}(\widetilde{X} F)\right\|_{j, k, \underline{\lambda}}=\sup _{Y \in \mathcal{U}_{k}(\mathfrak{g})} \sup _{x \in G} \frac{\left\|\widetilde{Y}_{x} R_{g}^{\star}(\widetilde{X} F)(x)\right\|_{j}}{\mu_{j}^{L_{j}}(x)|Y|_{k}} .
$$

Moreover, since for any $Y \in \mathcal{U}(\mathfrak{g})$ and $g \in G$, we have $R_{g^{-1}}^{\star} \tilde{Y} R_{g}^{\star}=\widehat{\operatorname{Ad}_{g^{-1}} Y}$ and since $F \in \mathcal{B} \underline{\mu}(G, \mathcal{E})$ and $\underline{\mu}$ has sub-multiplicative with degree $(\underline{L}, \underline{R})$, we get

$$
\begin{aligned}
\left\|\widetilde{X}_{g} R_{g}^{\star}(F)\right\|_{j, k, \underline{\lambda}} & =\sup _{Y \in \mathcal{U}_{k}(\mathfrak{g})} \sup _{x \in G} \frac{\left\|\left(\widehat{\operatorname{Ad}_{g^{-1}} Y} \tilde{X} F\right)(x g)\right\|_{j}}{\mu_{j}^{L_{j}}(x)|Y|_{k}} \\
& \leq C\|F\|_{j, k+k^{\prime}, \underline{\mu}}\left|\operatorname{Ad}_{g^{-1}}\right|_{k}|X|_{k^{\prime}} \sup _{x \in G} \frac{\mu_{j}(x g)}{\mu_{j}^{L_{j}}(x)} \\
& \leq\left. C\|F\|_{j, k+k^{\prime}, \underline{\mu}}\left|\operatorname{Ad}_{\left.g^{-1}\right|_{k}}\right| X\right|_{k^{\prime}} \mu_{j}^{R_{j}}(g),
\end{aligned}
$$

and one concludes using Lemma 1.14. 


\subsection{Tempered pairs}

In this section, we establish the main technical result of the first part of this memoir (Proposition 1.29), on which the construction of our oscillatory integral (and thus of our universal deformation formula for Fréchet algebras) essentially relies. To this aim, we start by introducing the class of tempered Lie groups (Definition [1.17) and the sub-class of tempered pairs (Definition [1.22). In Lemma 1.21] we give simple but important consequences for the modular weight, modular function and Haar measure, when the group is tempered. The rest of this section is devoted to the proof of Proposition 1.29 .

LEMma 1.16. Let $G$ be a connected real Lie group and $\psi: \mathbb{R}^{m} \rightarrow G$ be a global diffeomorphism. Then the multiplication and inverse operations seen through $\psi$ are tempered function 1 (in the ordinary sense of $\mathbb{R}^{m}$ ) if and only if for every element $A \in \mathcal{U}(\mathfrak{g})$ their derivatives along $\widetilde{A}$ are bounded by a function which is polynomial within the chart $\psi$.

Proof. Denote $m(x, y)=m_{x}(y)=\psi^{-1}(\psi(x) \cdot \psi(y))$ and $\iota(x)=\psi^{-1}\left(\psi(x)^{-1}\right)$ the multiplication and inverse of $G$ seen through $\psi \in \operatorname{Diff}\left(\mathbb{R}^{m}, G\right)$. Denote by $x_{e}$ the transportation of the neutral element $e$ of $G: \psi\left(x_{e}\right):=e$. Lastly, identifying naturally $T_{x_{e}}\left(\mathbb{R}^{m}\right)$ with $\mathbb{R}^{m}$, for every $X \in \mathbb{R}^{m}$ denote by

$$
\widetilde{X}_{x}^{\psi}:=m_{x \star x_{e}}(X)=\psi_{\star}^{-1}\left(\widetilde{\psi_{\star x_{e}} X}\right)_{\psi(x)},
$$

the left invariant vector field corresponding to $\psi_{\star_{x_{e}}} X \in \mathfrak{g}$.

Assume $m$ and $\iota$ are tempered in the usual sense. Then for $X \in \mathbb{R}^{m}$, by definition

$$
\widetilde{X}_{x}^{\psi}=\left.\frac{d}{d t} m\left(x, x_{e}+t X\right)\right|_{t=0},
$$

which is a linear combination of partial derivatives of $m$ all of them being bounded by some polynomials in $x$ since $m$ is tempered. In the same way, the derivatives of left-invariant vector fields are linear combinations of higher partial derivatives of compositions of $m$ with itself in the second variable, which are also bounded by some polynomials. Hence the left-invariant vector fields are tempered, and consequently so are the left-invariant derivatives of $m$ and $\iota$.

Conversely, assume $m$ and $\iota$ are tempered in the sense of left-invariant vector fields. We will see that the constant vector fields on $\mathbb{R}^{m}$ are linear combinations of left-invariant vector fields, the coefficients being tempered functions. Indeed, we have $X=\left(m_{x \star x_{e}}\right)^{-1}\left(\widetilde{X}_{x}^{\psi}\right)$ and the matrix elements of that inverse matrix are finite sums and products of the matrix elements of the original one, which are tempered, divided by its determinant. Thus all we have to check is that the inverse of the determinant is a tempered function. But $1 / \operatorname{det}\left(m_{x_{\star x_{e}}}\right)=\operatorname{det}\left(m_{\iota(x)_{\star x}}\right)$ is tempered since $m$ and $\iota$ are.

The preceding observation yields us to introduce the following notion:

Definition 1.17. A Lie group $G$ is called tempered if there exists a global coordinate system $\psi: \mathbb{R}^{m} \rightarrow G$ where the multiplication and inverse operations are tempered functions. A smooth function $f$ on a tempered Lie group is called a tempered function if $f \circ \psi$ is tempered.

\footnotetext{
${ }^{1}$ By tempered function, we mean a smooth function whose every derivative is bounded by a polynomial function. These functions are sometimes called "slowly increasing".
} 
REMARK 1.18. Every tempered Lie group, being diffeomorphic to an Euclidean space, is connected and simply connected. Moreover, by arguments similar to those of Lemma 1.16] a smooth function $f$ on a tempered Lie group is tempered if and only if for any $X \in \mathcal{U}(\mathfrak{g})$, its derivative along $\widetilde{X}$ is bounded by a polynomial function within the global chart $\mathbb{R}^{m} \rightarrow G$.

EXAMPLE 1.19. For any (simply connected) nilpotent Lie group, the exponential coordinates, $\mathfrak{g} \rightarrow G: X \mapsto \exp (X)$, provides $G$ with a structure of a tempered Lie group. Indeed, in the case of a nilpotent Lie group, the Baker-CampbellHausdorff series is finite.

REMARK 1.20. Observe also that we can replace in Lemma 1.16 left-invariant differential operators by right-invariant one. In fact, for a tempered group, leftinvariant vector fields are linear combinations of right-invariant one with tempered coefficients and vice versa.

LEMma 1.21. Let $G$ be a tempered Lie group. Then the modular weight $\mathfrak{d}_{G}$ and the modular function $\Delta_{G}$ are tempered. Moreover, in the transported coordinates, every Haar measure on $G$ is a multiple of a Lebesgue measure on $\mathbb{R}^{m}$ by a tempered density.

Proof. The conjugate action $\mathbf{C}: G \times G \rightarrow G:(g, x) \mapsto g x g^{-1}$ is a tempered map when read in the global coordinate system. Therefore, the evaluation of the restriction of its tangent mapping to the first factor $G \times\{e\}$ on the constant section $0 \oplus X, X \in \mathfrak{g}$, of $T(G \times G)$ consists of a tempered mapping:

$$
G \rightarrow \mathfrak{g}, \quad g \mapsto \mathbf{C}_{\star(g, e)}\left(0_{g} \oplus X\right) .
$$

The latter coincides with $g \mapsto \operatorname{Ad}_{g}(X)$. Varying $X$ in the finite dimensional vector space $\mathfrak{g}$, yields the tempered map Ad $: G \rightarrow \operatorname{End}(\mathfrak{g})$. This shows that $\mathfrak{d}_{G}$ is tempered.

Transporting the group structure of $G$ to $\mathbb{R}^{n}$ by means of the global coordinates, it is clear that any Haar measure on $\mathbb{R}^{n}$ (for the transported group law) is absolutely continuous with respect to the Lebesgue measure. Let $\mathrm{d}_{G}(\xi)$ be a left invariant Haar measure on $G$ transported to $\mathbb{R}^{n}$. Let also $\rho: \mathbb{R}^{n} \rightarrow \mathbb{R}$ be the Radon-Nikodym derivative of $\mathrm{d}_{G}(\xi)$ with respect to $\mathrm{d} \xi$, the Lebesgue measure on $\mathbb{R}^{n}$. Let $\xi_{e} \in \mathbb{R}^{n}$ be the transported neutral element of $G$. By left-invariance of the Haar measure $\mathrm{d}_{G}(\xi)$, we get

$$
\rho\left(m\left(\xi^{\prime}, \xi\right)\right)=\rho(\xi)\left|\operatorname{Jac}_{L_{\xi^{\prime}}^{\star}}\right|(\xi), \quad \forall \xi, \xi^{\prime} \in \mathbb{R}^{n},
$$

where $m(.,$.$) denotes the transported multiplication law on \mathbb{R}^{n}$ and $L_{\xi}^{\star}$ stands for the associated left translation operator on $\mathbb{R}^{n}$. Letting $\xi \rightarrow \xi_{e}$, we deduce

$$
\rho(\xi)=\rho\left(\xi_{e}\right)\left|\operatorname{Jac}_{L_{\xi}^{\star}}\right|\left(\xi_{e}\right), \quad \forall \xi \in \mathbb{R}^{n},
$$

and we conclude by Lemma 1.16 using the fact that the multiplication law is tempered.

Next, we let $\iota$ the inversion map of $G$ transported to $\mathbb{R}^{n}$. We have in the transported coordinates:

$\Delta_{G}(\xi)=\frac{\mathrm{d}_{G}(\iota(\xi))}{\mathrm{d}_{G}(\xi)}=\frac{\mathrm{d}_{G}(\iota(\xi))}{\mathrm{d} \xi} \frac{\mathrm{d} \xi}{\mathrm{d}_{G}(\xi)}=\operatorname{Jac}_{\iota}(\xi) \frac{\mathrm{d}_{G}(\iota(\xi))}{\mathrm{d}(\iota(\xi))} \frac{\mathrm{d} \xi}{\mathrm{d}_{G}(\xi)}=\operatorname{Jac}_{\iota}(\xi) \frac{\rho(\iota(\xi))}{\rho(\xi)}$,

and we conclude using what precedes and the temperedness of the inversion map on $G$. 
We now consider the data of a pair $(G, S)$ where $G$ is a connected real Lie group with real Lie algebra $\mathfrak{g}$ and $S$ is a real-valued smooth function on $G$.

Definition 1.22. The pair $(G, S)$ is called tempered if the following two properties are satisfied:

(i) The map

$$
\phi: G \rightarrow \mathfrak{g}^{\star}, \quad x \mapsto[\mathfrak{g} \rightarrow \mathbb{R}, X \mapsto(\widetilde{X} . S)(x)],
$$

is a global diffeomorphism.

(ii) The inverse map $\phi^{-1}: \mathfrak{g}^{\star} \simeq \mathbb{R}^{m} \rightarrow G$ endows $G$ with the structure of a tempered Lie group.

REMARK 1.23. Within the above situation, the function $S$ is itself automatically tempered. Indeed, in the proof of Lemma 1.16, we have seen that directional derivatives in the coordinate system $\phi$ are expressed as linear combinations of left-invariant vector fields with tempered coefficients. Hence, within a basis $\left\{X_{j}\right\}_{j=1, \ldots, N}$ of $\mathfrak{g}$, denoting $x_{j}=\left(\widetilde{X_{j}} S\right)(x)$, we have $\partial_{x_{j}} S(x)=\sum_{k} m_{j}^{k}(x) x_{k}$ where the matrix $\left(m_{j}^{k}\right)_{j, k}$ have tempered entries. This implies that the partial derivatives (of every strictly positive order) of $S$ are tempered. In spherical coordinates $(r, \theta)$ (associated to the $x_{j}$ 's) one observes that $\partial_{r} S(r, \theta)=R(\theta)_{1}^{k} \partial_{x_{k}} S$ where $\theta$ belongs to the unit sphere $S^{N-1}$ and where $R(\theta)$ is a (rotation) matrix that smoothly depends on $\theta$. Hence:

$$
|S(x)|=\left|C+\int_{r_{0}}^{r} \partial_{\rho} S(\rho, \theta) \mathrm{d} \rho\right| \leq|C|+\int_{r_{0}}^{r}\left|R(\theta)_{1}^{k}\right| \rho^{n_{k}} \mathrm{~d} \rho,
$$

which for large $x$ is smaller than a multiple of some positive power of $r$. Therefore the function $S$ has polynomial growth too.

Given a tempered pair $(G, S)$, with $\mathfrak{g}$ the Lie algebra of $G$, we now consider a vector space decomposition:

$$
\mathfrak{g}=\bigoplus_{n=0}^{N} V_{n}
$$

and for every $n=0, \ldots, N$, an ordered basis $\left\{e_{j}^{n}\right\}_{j=1, \ldots, \operatorname{dim}\left(V_{n}\right)}$ of $V_{n}$. We get global coordinates on $G$ :

$$
x_{n}^{j}:=\left(\widetilde{e_{j}^{n}} . S\right)(x), \quad n=0, \ldots, N, \quad j=1, \ldots, \operatorname{dim}\left(V_{n}\right) .
$$

We choose a scalar product on each $V_{n}$ and let $|\cdot|_{n}$ be the associated Euclidean norm. Given an element $A \in \mathcal{U}(\mathfrak{g})$, we let $\widetilde{A}^{*}$ be the formal adjoint of the left-invariant differential operator $\widetilde{A}$, with respect to the inner product of $L^{2}(G)$. We make the obvious observation that $\widetilde{A}^{*}$ is still left-invariant. Indeed, for $\psi, \varphi \in C_{c}^{\infty}(G)$ and $g \in G$, since $L_{g}^{\star}$ is unitary on $L^{2}(G)$, we have

$$
\left\langle L_{g}^{\star} \widetilde{A}^{*} \psi, \varphi\right\rangle=\left\langle\psi, \widetilde{A} L_{g^{-1}}^{\star} \varphi\right\rangle=\left\langle\psi, L_{g^{-1}}^{\star} \widetilde{A} \varphi\right\rangle=\left\langle\widetilde{A}^{*} L_{g}^{\star} \psi, \varphi\right\rangle .
$$

Moreover, we make the following requirement of compatibility of the adjoint map on $L^{2}(G)$ with respect to the ordered decomposition (1.13). Namely, denoting for every $n \in\{0, \ldots, N\}$ :

$$
V^{(n)}:=\bigoplus_{k=0}^{n} V_{k}
$$


we assume:

$$
\forall n=0, \ldots, N, \quad \forall A \in \mathcal{U}\left(V_{n}\right), \quad \exists B \in \mathcal{U}\left(V^{(n)}\right) \quad \text { such that } \quad \widetilde{A}^{*}=\widetilde{B},
$$

where the space $\mathcal{U}\left(V^{(n)}\right)$ is the subalgebra of $\mathcal{U}(\mathfrak{g})$ generated by $V^{(n)}$, as defined in (0.8). We now pass to regularity assumptions regarding the function $S$.

DEFinition 1.24. Set

$$
\mathbf{E}:=\exp \{i S\}
$$

A tempered pair $(G, S)$ is called admissible, if there exists a decomposition (1.13) with associated coordinate system (1.14), such that for every $n=0, \ldots, N$, there exists an element $X_{n} \in \mathcal{U}\left(V_{n}\right) \subset \mathcal{U}(\mathfrak{g})$ whose associated multiplier $\alpha_{n}$, defined as

$$
\widetilde{X}_{n} \mathbf{E}=: \alpha_{n} \mathbf{E}
$$

satisfies the following properties:

(i) There exist $C_{n}>0$ and $\rho_{n}>0$ such that:

$$
\left|\alpha_{n}\right| \geq C_{n}\left(1+\left|x_{n}\right|_{n}^{\rho_{n}}\right)
$$

where $x_{n}:=\left(x_{n}^{j}\right)_{j=1, \ldots, \operatorname{dim}\left(V_{n}\right)}$.

(ii) For all $n=0, \ldots, N$, there exists a tempered function $\mu_{n} \in C^{\infty}\left(G, \mathbb{R}_{+}^{*}\right)$ such that:

(ii.1) For every $A \in \mathcal{U}\left(V^{(n)}\right) \subset \mathcal{U}(\mathfrak{g})$ there exists $C_{A}>0$ such that:

$$
\left|\widetilde{A} \alpha_{n}\right| \leq C_{A}\left|\alpha_{n}\right| \mu_{n} .
$$

(ii.2) $\mathrm{n} \mu_{n}$ is independent of the variables $\left\{x_{r}^{j}\right\}_{j=1, \ldots, \operatorname{dim}\left(V_{r}\right)}$, for all $r \leq n$ :

$$
\frac{\partial \mu_{n}}{\partial x_{r}^{j}}=0, \quad \forall r \leq n, \forall j=1, \ldots, \operatorname{dim}\left(V_{r}\right) .
$$

We now give a sequence of Lemmas allowing the construction of a sequence of differential operator $\left\{\mathbf{D}_{j}\right\}_{j \in \mathbb{N}}$ such that, for $\underline{\mu}$ a family of tempered weights, $\mathbf{D}_{j}$ sends continuously $\mathcal{B}^{\mu_{j}}\left(G, \mathcal{E}_{j}\right)$ to $L^{1}\left(G, \mathcal{E}_{j}\right)$, where $\mathcal{E}_{j}$ is the semi-normed space $\left(\mathcal{E},\|\cdot\|_{j}\right)$ and which is such that $\mathbf{D}_{j}^{*} \mathbf{E}=\mathbf{E}$. Thus is all that we need to construct our oscillatory integral. We start with a preliminary result, which gives an upper bound for powers of derivatives of the inverse of a multiplier, in the context of admissible tempered pairs.

Lemma 1.25. Fix $n=0, \ldots, N$. Let $\alpha \in C^{\infty}(G)$ be non-vanishing and $1 \leq \mu \in$ $C^{\infty}(G)$ such that for every $A \in \mathcal{U}\left(V^{(n)}\right)$ there exists $C>0$ with $|\widetilde{A} \alpha| \leq C \mu|\alpha|$. Fixing $X \in \mathcal{U}\left(V^{(n)}\right)$, a monomial of homogeneous degree $M \in \mathbb{N}$, we consider the differential operator

$$
D_{X, \alpha}: C^{\infty}(G) \rightarrow C^{\infty}(G), \quad \Phi \mapsto \widetilde{X}\left(\frac{\Phi}{\alpha}\right) .
$$

Then, for every $r \in \mathbb{N}$, there exist an element $X^{\prime} \in \mathcal{U}(\mathfrak{g})$ of maximal homogeneous degree bounded by $r M$ and a constant $C>0$ such that for every $\Phi \in C^{\infty}(G)$ we have:

$$
\left|D_{X, \alpha}^{r} \Phi\right| \leq C \frac{\mu^{r^{2} M}}{|\alpha|^{r}}\left|\tilde{X}^{\prime} \Phi\right|
$$


Proof. We start by recalling Faà di Bruno's formula:

$$
\frac{\mathrm{d}^{r}}{\mathrm{~d} t^{r}}\left(\frac{1}{\mathbf{f}}\right)=\frac{1}{\mathbf{f}} \sum_{\vec{M}} C_{\vec{M}}^{r} \prod_{j=1}^{r}\left(\frac{\mathbf{f}^{(j)}}{\mathbf{f}}\right)^{M_{j}}, \quad \mathbf{f} \in C^{\infty}(\mathbb{R}),
$$

where $\vec{M}=\left(M_{1}, \ldots, M_{r}\right)$ runs along partitions of $r$ (i.e. $\left.r=\sum_{j=1}^{r} j M_{j}\right)$ and where $C_{\vec{M}}^{r}$ is some combinatorial coefficient. Within Sweedler's notations (0.2), Faà di Bruno formula then yields for $\Phi \in C^{\infty}(G)$ :

$$
D_{X, \alpha} \Phi=\sum_{(X)}\left(\tilde{X}_{(1)} \frac{1}{\alpha}\right)\left(\tilde{X}_{(2)} \Phi\right)=\sum_{(X)} \frac{1}{\alpha} \sum \prod\left(\frac{\tilde{X}_{j} \alpha}{\alpha}\right)^{M_{j}}\left(\widetilde{X}_{(2)} \Phi\right)
$$

where the second sum and product run over partitions of $M_{(1)}:=\operatorname{deg}\left(X_{(1)}\right) \leq M$ and where the element $X_{j}$ is of homogeneous degree $j=1, \ldots, r$ and contains the combinatorial coefficients of Faà di Bruno's formula. Of course, we also have that $X_{(1)}, X_{(2)}$ and $X_{j}$ all belong to $\mathcal{U}\left(V^{(n)}\right)$. Thus, $\left|\widetilde{X}_{j} \alpha\right| \leq C(X) \mu|\alpha|$, the estimation is satisfied for $r=1$. For $r=2$, we observe:

$$
\begin{aligned}
D_{X, \alpha}^{2} \Phi & =\sum_{(X)}\left(\tilde{X}_{(1)} \frac{1}{\alpha}\right) \tilde{X}_{(2)}\left(\sum_{(X)} \tilde{X}_{(1)} \frac{1}{\alpha} \tilde{X}_{(2)} \Phi\right) \\
& =\sum_{(X),\left(X_{(2)}\right)}\left(\tilde{X}_{(1)} \frac{1}{\alpha}\right) \sum_{(X)}\left(\tilde{X}_{(21)} \tilde{X}_{(1)} \frac{1}{\alpha}\right)\left(\tilde{X}_{(22)} \tilde{X}_{(2)} \Phi\right) .
\end{aligned}
$$

Faà di Bruno's formula for $\frac{1}{\alpha}$ then yields the assertion for $r=2$. Iterating this procedure, we get that

$$
D_{X, \alpha}^{r} \Phi=\sum_{(X)} \prod_{j=1}^{r}\left(\tilde{X}^{(j)} \frac{1}{\alpha}\right)\left(\tilde{X}^{\prime} \Phi\right)
$$

for some elements $X^{(j)}, X^{\prime} \in \mathcal{U}\left(V^{(n)}\right)$ where the maximal homogeneous degree of $X^{(j)}$ is bounded by $j M$. (We have absorbed the combinatorial coefficients of Faà di Bruno' s formula in $X^{(j)}$ and $X^{\prime}$.) Therefore, Faà di Bruno's formula yields for every $j=1, \ldots, r$ :

$$
\begin{aligned}
\left|\widetilde{X}^{(j)} \frac{1}{\alpha}\right|=\left|\frac{1}{\alpha} \sum \prod\left(\frac{\widetilde{X}_{k}^{(j)} \cdot \alpha}{\alpha}\right)^{\operatorname{deg}\left(X_{k}^{(j)}\right)}\right| & \leq C \frac{\mu^{\sum_{k} \operatorname{deg}\left(X_{k}^{(j)}\right)}}{|\alpha|} \\
& \leq C \frac{\mu^{\operatorname{deg}\left(X^{(j)}\right)}}{|\alpha|} \leq C \frac{\mu^{j M}}{|\alpha|} .
\end{aligned}
$$

Therefore since $\frac{r(r+1)}{2} \leq r^{2}$ and $\mu \geq 1$, we get the (rough) estimation:

$$
\left|D_{X, \alpha}^{r} \Phi\right| \leq C \sum_{(X)} \prod_{j=1}^{r} \frac{1}{|\alpha|} \mu^{j M}\left|\tilde{X}^{\prime} \Phi\right| \leq C^{\prime} \sum_{(X)} \frac{1}{|\alpha|^{r}} \mu^{r^{2} M}\left|\tilde{X}^{\prime} \Phi\right|,
$$

which delivers the proof.

We now fix an admissible tempered pair $(G, S)$ and for all $n=0, \ldots, N$, we let $X_{n} \in \mathcal{U}\left(V_{n}\right)$ as given in Definition 1.24 and we let $\alpha_{n}, \mu_{n} \in C^{\infty}(G)$ be the 
associated multiplier and tempered function. Accordingly to the previous notations, we introduce the differential operators:

$$
D_{n}:=D_{X_{n}^{*}, \alpha_{n}}: C^{\infty}(G) \rightarrow C^{\infty}(G), \quad \Phi \mapsto \widetilde{X}_{n}^{*}\left(\frac{\Phi}{\alpha_{n}}\right) .
$$

Recall that by assumption, there exists $Y_{n} \in \mathcal{U}\left(V^{(n)}\right)$ such that $\widetilde{X}_{n}^{*}=\widetilde{Y}_{n}$ and thus, we can apply Lemma 1.25 to these operators. For every $r_{n} \in \mathbb{N}$, accordingly to the expression (1.18), we write for $\Phi \in C^{\infty}(G)$ :

$$
D_{n}^{r_{n}} \Phi=\sum_{\left(X_{n}\right)} \prod_{j=1}^{r_{n}}\left(\widetilde{X}_{n}^{(j)} \frac{1}{\alpha_{n}}\right)\left(\widetilde{X}_{n}^{\prime} \Phi\right)
$$

where $X_{n}^{(j)} \in \mathcal{U}\left(V^{(n)}\right)$ and its homogeneous degree is bounded by $j M_{n}$, with $M_{n}$ the maximal homogeneous degree of $X_{n}$ and where the one of $X_{n}^{\prime}$ is bounded by $r_{n} M_{n}$. Setting

$$
\Psi_{n}=\prod_{j=1}^{r_{n}}\left(\widetilde{X}_{n}^{(j)} \frac{1}{\alpha_{n}}\right)
$$

we then write (abusively since in fact it is a finite sum of such terms):

$$
D_{n}^{r_{n}}=: \Psi_{n} \tilde{X}_{n}^{\prime}
$$

Given a $N+1$-tuple of integers $\vec{r}=\left(r_{0}, \ldots, r_{N}\right)$, we will be led to consider the operator

$$
\mathbf{D}_{\vec{r}}:=\mathbf{D}_{r_{0}, \ldots, r_{N}}:=D_{0}^{r_{0}} D_{1}^{r_{1}} \ldots D_{N}^{r_{N}} .
$$

Using the iterated Sweedler's notation (0.3), a recursive use of (1.21) yields

$$
\begin{aligned}
\mathbf{D}_{\vec{r}}= & \sum_{\left(X_{0}^{\prime}\right)} \sum_{\left(X_{1}^{\prime}\right)} \cdots \sum_{\left(X_{N-2}^{\prime}\right)} \sum_{\left(X_{N-1}^{\prime}\right)} \\
& {\left[\Psi_{0}\right]\left[\left(\widetilde{X}_{0}^{\prime}\right)_{(1)} \Psi_{1}\right]\left[\left(\widetilde{X}_{0}^{\prime}\right)_{(21)}\left(\widetilde{X}_{1}^{\prime}\right)_{(1)} \Psi_{2}\right]\left[\left(\widetilde{X}_{0}^{\prime}\right)_{(221)}\left(\widetilde{X}_{1}^{\prime}\right)_{(21)}\left(\widetilde{X}_{2}^{\prime}\right)_{(1)} \Psi_{3}\right] \ldots } \\
& {\left[\left(\widetilde{X}_{0}^{\prime}\right)_{(22 \ldots 21)}\left(\widetilde{X}_{1}^{\prime}\right)_{(2 \ldots 21)} \ldots\left(\widetilde{X}_{N-3}^{\prime}\right)_{(21)}\left(\widetilde{X}_{N-2}^{\prime}\right)_{(1)} \Psi_{N-1}\right] } \\
& {\left[\left(\widetilde{X}_{0}^{\prime}\right)_{(222 \ldots 21)}\left(\widetilde{X}_{1}^{\prime}\right)_{(22 \ldots 21)} \ldots\left(\widetilde{X}_{N-3}^{\prime}\right)_{(221)}\left(\widetilde{X}_{N-2}^{\prime}\right)_{(21)}\left(\widetilde{X}_{N-1}^{\prime}\right)_{(1)} \Psi_{N}\right] } \\
& \left(\widetilde{X}_{0}^{\prime}\right)_{(222 \ldots 2)}\left(\widetilde{X}_{1}^{\prime}\right)_{(22 \ldots 2)} \ldots\left(\widetilde{X}_{N-3}^{\prime}\right)_{(222)}\left(\widetilde{X}_{N-2}^{\prime}\right)_{(22)}\left(\widetilde{X}_{N-1}^{\prime}\right)_{(2)} \widetilde{X}_{N}^{\prime} .
\end{aligned}
$$

Set then,

$$
\begin{aligned}
\Psi_{1,0} & :=\left(\widetilde{X}_{0}^{\prime}\right)_{(1)} \Psi_{1} \\
\Psi_{2,1,0} & :=\left(\widetilde{X}_{0}^{\prime}\right)_{(21)}\left(\widetilde{X}_{1}^{\prime}\right)_{(1)} \Psi_{2} \\
\Psi_{3,2,1,0} & :=\left(\widetilde{X}_{0}^{\prime}\right)_{(221)}\left(\widetilde{X}_{1}^{\prime}\right)_{(21)}\left(\widetilde{X}_{2}^{\prime}\right)_{(1)} \Psi_{3} \\
& \vdots \\
\Psi_{N-1, \ldots, 1,0} & :=\left(\widetilde{X}_{0}^{\prime}\right)_{(22 \ldots 21)}\left(\widetilde{X}_{1}^{\prime}\right)_{(2 \ldots 21)} \ldots\left(\widetilde{X}_{N-3}^{\prime}\right)_{(21)}\left(\widetilde{X}_{N-2}^{\prime}\right)_{(1)} \Psi_{N-1} \\
\Psi_{N, N-1, \ldots, 1,0} & :=\left(\widetilde{X}_{0}^{\prime}\right)_{(222 \ldots 21)}\left(\widetilde{X}_{1}^{\prime}\right)_{(22 \ldots 21)} \ldots\left(\widetilde{X}_{N-2}^{\prime}\right)_{(21)}\left(\widetilde{X}_{N-1}^{\prime}\right)_{(1)} \Psi_{N}
\end{aligned}
$$

and

$$
X_{N, \ldots, 0}^{\prime}:=\left(X_{0}^{\prime}\right)_{(222 \ldots 2)}\left(X_{1}^{\prime}\right)_{(22 \ldots 2)} \ldots\left(X_{N-3}^{\prime}\right)_{(222)}\left(X_{N-2}^{\prime}\right)_{(22)}\left(X_{N-1}^{\prime}\right)_{(2)} X_{N}^{\prime}
$$


in terms of which we have (with the same abuse of notations as in (1.22) above):

$$
\mathbf{D}_{\vec{r}}=\Psi_{0} \Psi_{1,0} \Psi_{2,1,0} \Psi_{3,2,1,0} \ldots \Psi_{N-1, \ldots, 1,0} \Psi_{N, \ldots, 0} \widetilde{X}_{N, N-1, \ldots, 1,0}^{\prime} .
$$

Lemma 1.26. Fix $n=0, \ldots, N$ and let $\alpha \in C^{\infty}(G)$ and $\mu \in C^{\infty}\left(G, \mathbb{R}_{+}^{*}\right)$ satisfying the hypothesis of Lemma 1.25. For $j=1, \ldots, r$ and $r \in \mathbb{N}^{*}$, fix also $X^{(j)} \in \mathcal{U}\left(V^{(n)}\right)$ and define

$$
\Psi:=\prod_{j=1}^{r}\left(\widetilde{X}^{(j)} \frac{1}{\alpha}\right)
$$

where $\operatorname{deg}\left(X^{(j)}\right) \leq j M$, for a given $M \in \mathbb{N}^{*}$. Consider a monomial $Y \in \mathcal{U}\left(V^{(n)}\right)$, then we have

$$
\widetilde{Y} \Psi=\sum_{(Y)} \prod_{j=1}^{r}\left(\widetilde{Y}^{(j)} \frac{1}{\alpha}\right) \quad \text { with } \quad \operatorname{deg}\left(Y^{(j)}\right) \leq j M+\operatorname{deg}(Y),
$$

and moreover there exists $C>0$ such that

$$
|\widetilde{Y} \Psi| \leq C \frac{\mu^{r^{2} M+r \operatorname{deg}(Y)}}{|\alpha|^{r}} .
$$

Proof. The equality is immediate. Regarding the inequality, we first note that by virtue of Faà di Bruno's formula, we have for a finite sum:

$$
\tilde{Y}^{(j)} \frac{1}{\alpha}=\frac{1}{\alpha} \sum \prod_{k=1}^{\operatorname{deg}\left(Y^{(j)}\right)}\left(\frac{\widetilde{Y}_{k}^{(j)} \alpha}{\alpha}\right)^{\operatorname{deg}\left(Y_{k}^{(j)}\right)} .
$$

Hence

$$
\left|\widetilde{Y}^{(j)} \frac{1}{\alpha}\right| \leq C \frac{\mu^{\sum_{k} \operatorname{deg}\left(Y_{k}^{(j)}\right)}}{|\alpha|} \leq C \frac{\mu^{\operatorname{deg}\left(Y^{(j)}\right)}}{|\alpha|} \leq C \frac{\mu^{j M+\operatorname{deg}(Y)}}{|\alpha|} .
$$

We then conclude as in the proof of Lemma 1.25

From the lemmas above, we deduce an estimate for the 'coefficient functions' appearing in the expression of the differential operator $\mathbf{D}_{\vec{r}}$ in (1.24).

Corollary 1.27. Let $(G, S)$ be an admissible tempered pair with decomposition $\mathfrak{g}=\bigoplus_{n=0}^{N} V_{n}$ and accordingly to Definition 1.24. for $n=0, \ldots, N$, we let $\left(X_{n}, \alpha_{n}, \mu_{n}\right) \in \mathcal{U}\left(V_{n}\right) \times C^{\infty}(G) \times C^{\infty}(G)$ be the associated differential operator, multiplier and tempered function. Then, for $k=0, \ldots, N$ and $r_{k} \in \mathbb{N}^{*}$, with $\Psi_{k, \ldots, 0} \in C^{\infty}(G)$ defined in (1.23), we have

$$
\left|\Psi_{k, \ldots, 0}\right| \leq C_{k} \frac{\mu_{k}^{r_{k}^{2} M_{k}+r_{k} \sum_{j=0}^{k-1} r_{j} M_{j}}}{\left|\alpha_{k}\right|^{r_{k}}},
$$

for some finite non-negative constant $C_{k}$ and where $M_{n}:=\operatorname{deg}\left(X_{n}\right), n=0, \ldots, N$.

Proof. Observe that

$$
\Psi_{k, \ldots, 0}=\prod_{j=0}^{k-1}\left(\widetilde{X_{j}^{\prime}}\right)_{(2 \ldots 21)} \Psi_{k}
$$

where $\Psi_{k}$ is defined in (1.20). Since $\left(X_{j}^{\prime}\right)_{(2 \ldots 21)} \in \mathcal{U}\left(V^{(k)}\right)$ with homogeneous degree of is bounded by $r_{j} M_{j}$ for every $j=0, \ldots, k-1$, the estimate we need follows from Lemma 1.26.

We can now state the main technical results of this chapter. 
Proposition 1.28. Let $(G, S)$ be an admissible tempered pair and let $\mu$ be a tempered weight. Then, there exists $\vec{r}=\left(r_{0}, \ldots, r_{N}\right) \in \mathbb{N}^{N+1}$ such that for every element $F \in \mathcal{B}^{\mu}(G)$, the function $\mathbf{D}_{\vec{r}} F$ belongs to $L^{1}(G)$. More precisely, there exist a finite constant $C>0$ and $K \in \mathbb{N}$ with $K \leq \sum_{k=0}^{N} r_{k} M_{k}$ and $M_{k}=\operatorname{deg}\left(X_{k}\right)$ (with $X_{k} \in \mathcal{U}_{M_{k}}(\mathfrak{g})$ as given in Definition 1.24), such that for all $F \in \mathcal{B}^{\mu}(G)$, we have:

$$
\left\|\mathbf{D}_{\vec{r}} F\right\|_{1} \leq C \sup _{X \in \mathcal{U}_{K}(\mathfrak{g})} \sup _{g \in G}\left\{\frac{|\widetilde{X} F(x)|}{\mu(g)|X|_{K}}\right\}=C\|F\|_{K, \mu} .
$$

Proof. By Lemma 1.21, in the coordinates (1.14), the Radon-Nikodym derivative of the left Haar measure on $G$ with respect to the Lebesgue measure on $\mathfrak{g}^{\star}$, is bounded by a polynomial in $\left\{x_{n}^{j}, j=1, \ldots, \operatorname{dim}\left(V_{n}\right), n=0, \ldots, N\right\}$. By the assumption of temperedness of the weight $\mu$, the latter is also bounded by a polynomial in the same coordinates. Now, observe from (1.24), that we have for any $\vec{r}=\left(r_{1}, \ldots, r_{N}\right)$ and for $K=\operatorname{deg}\left(X_{N, \ldots, 0}^{\prime}\right) \leq \sum_{k=0}^{N} r_{k} M_{k}$ :

$$
\begin{aligned}
\left|\mathbf{D}_{\vec{r}} F\right| & \leq\left|\Psi_{0}\right|\left|\Psi_{1,0}\right|\left|\Psi_{2,1,0}\right| \ldots\left|\Psi_{N, \ldots, 0}\right|\left|\widetilde{X}_{N, \ldots, 0}^{\prime} F\right| \\
& \leq\left|\Psi_{0}\right|\left|\Psi_{1,0}\right|\left|\Psi_{2,1,0}\right| \ldots\left|\Psi_{N, \ldots, 0}\right| \mu\left|X_{N, \ldots, 0}^{\prime}\right|_{K}\|F\|_{K, \mu} .
\end{aligned}
$$

This will gives the estimate, if we prove that the function in front of $\|F\|_{K, \mu}$ in (1.25) is integrable for a suitable choice of $\vec{r} \in \mathbb{N}^{N+1}$. We prove a stronger result, namely that given $\vec{R}=\left(R_{0}, \ldots, R_{N}\right) \in \mathbb{N}^{N+1}$, there exists $\vec{r}=\left(r_{0}, \ldots, r_{N}\right) \in \mathbb{N}^{N+1}$ such that the associated functions $\Psi_{k, \ldots, 0}$ (which depend on $\vec{r}$ ) satisfy:

$$
\left|\Psi_{0}(x)\right|\left|\Psi_{1,0}(x)\right|\left|\Psi_{2,1,0}(x)\right| \ldots\left|\Psi_{N, \ldots, 0}(x)\right| \leq \frac{C}{\left(1+\left|x_{0}\right|\right)^{R_{0}} \ldots\left(1+\left|x_{N}\right|\right)^{R_{N}}} .
$$

From Corollary 1.27 and writing $r_{k}^{2} M_{k}+r_{k} \sum_{j=0}^{k-1} r_{j} M_{j}=r_{k} \sum_{j=0}^{k} r_{j} M_{j}$, we obtain the following estimation:

$$
\left|\Psi_{0}\right|\left|\Psi_{1,0}\right|\left|\Psi_{2,1,0}\right| \ldots\left|\Psi_{N, \ldots, 0}\right| \leq C \prod_{k=0}^{N} \frac{\mu_{k}^{r_{k} \sum_{j=0}^{k} r_{j} M_{j}}}{\alpha_{k}^{r_{k}}} .
$$

Moreover, by assumption of temperedness, see Definition 1.24 (ii.1), there exist $\rho_{0}, \ldots, \rho_{N}>0$ such that

$$
\left|\Psi_{0}(x)\right|\left|\Psi_{1,0}(x)\right|\left|\Psi_{2,1,0}(x)\right| \ldots\left|\Psi_{N, \ldots, 0}(x)\right| \leq C \prod_{k=0}^{N} \frac{\mu_{k}(x)^{r_{k} \sum_{j=0}^{k} r_{j} M_{j}}}{\left(1+\left|x_{k}\right|\right)^{\rho_{k} r_{k}}} .
$$

From the hypothesis of Definition 1.24 (ii.2), we deduce that the element $\mu_{N}$ is constant. Indicating the variable dependence into parentheses, one also has

$$
\begin{aligned}
\mu_{N-1} & =\mu_{N-1}\left(x_{N}\right), \quad \mu_{N-2}=\mu_{N-2}\left(x_{N-1}, x_{N}\right), \quad \ldots \\
\mu_{1} & =\mu_{1}\left(x_{2}, \ldots, x_{N}\right), \quad \mu_{0}=\mu_{0}\left(x_{1}, x_{2}, \ldots, x_{N}\right) .
\end{aligned}
$$

Denoting by $m_{n}, n=0, \ldots, N$, the degree of a polynomial function that, in the variables (1.14), dominates the tempered function $\mu_{n}$, we obtain the sufficient conditions:

$$
r_{0} \rho_{0} \geq R_{0} \quad \text { and } \quad \rho_{n} r_{n}-\sum_{k=0}^{n-1}\left(m_{k} r_{k} \sum_{j=0}^{k} r_{j} M_{j}\right) \geq R_{n}, \quad n=1, \ldots, N,
$$

which are always achievable. 
Let now $\mathcal{E}$ be a complex Fréchet space, with topology associated with a countable family of semi-norms $\left\{\|\cdot\|_{j}\right\}_{j \in \mathbb{N}}$. An immediate modification of its proof, lead us to the following version of Proposition 1.28 (the only difference with the former is that now the index $K \in \mathbb{N}$ may depend on $j$ via the order of the tempered weight $\left.\mu_{j}\right)$.

Proposition 1.29. Let $(G, S)$ be an admissible tempered pair, $\mathcal{E}$ be a complex Fréchet space and let $\underline{\mu}$ be a family of tempered weights. Then for all $j \in \mathbb{N}$, there exist $\vec{r}_{j} \in \mathbb{N}^{N+1}, C_{j}>0$ and $k_{j} \in \mathbb{N}$, such that for every element $F \in \mathcal{B} \underline{\mu}(G, \mathcal{E})$, we have

$$
\int_{G}\left\|\mathbf{D}_{\vec{r}_{j}} F(g)\right\|_{j} \mathrm{~d}_{G}(g) \leq C_{j} \sup _{X \in \mathcal{U}_{K_{j}}(\mathfrak{g})} \sup _{g \in G}\left\{\frac{\|\widetilde{X} F(x)\|_{j}}{\mu_{j}(g)|X|_{k_{j}}}\right\}=: C_{j}\|F\|_{j, k_{j}, \underline{\mu}} .
$$

\subsection{An oscillatory integral for admissible tempered pairs}

Our notion of oscillatory integral is an immediate consequence of Proposition 1.29 together with the following immediate but essential observation:

Proposition 1.30. Let $(G, S)$ be an admissible tempered pair, let $\mathbf{E}$ be the function on $G$ defined in (1.17) and, for $\vec{r} \in \mathbb{N}^{N+1}$, let $\mathbf{D}_{\vec{r}}$ be the differential operator given in (1.22). Then, with $\mathbf{D}_{\vec{r}}^{*}$ the formal adjoint of $\mathbf{D}_{\vec{r}}$ with respect to the inner product of $L^{2}(G)$, we have:

$$
\mathbf{D}_{\vec{r}}^{*} \mathbf{E}=\mathbf{E}, \quad \forall \vec{r} \in \mathbb{N}^{N+1},
$$

Proof. By construction, we have

$$
\mathbf{D}_{\vec{r}}=D_{0}^{r_{0}} D_{1}^{r_{1}} \ldots D_{N}^{r_{N}}
$$

where the operators $D_{n}, n=0, \ldots, N$, are given in (1.19). Hence it suffices to prove the identity above for $\vec{r}=(0, \ldots, 0,1,0, \ldots, 0)$. But this immediately follows by construction since

$$
D_{n}^{*}=\frac{1}{\alpha_{n}} \widetilde{X}_{n} \quad \text { and } \quad \widetilde{X}_{n} \mathbf{E}=\alpha_{n} \mathbf{E} .
$$

This completes the proof.

Definition 1.31. Let $(G, S)$ be an admissible tempered pair, $\mu$ a tempered weight, $\mathbf{m}$ an element of $\mathcal{B}^{\mu}(G)$ and $\mu, \hat{\mu}$ two families of tempered weights such that $\underline{\mu} \prec \hat{\mu}$ (hence $\underline{\mu} . \mu \prec \hat{\mu} . \mu$ ). Associated to the family of weights $\hat{\mu} . \mu$, let $\vec{r}_{j} \in \mathbb{N}^{N+1}$, $\bar{j} \in \overline{\mathbb{N}}$, as given in Proposition 1.29 and let $\mathbf{D}_{\vec{r}_{j}}$ be the differential operators given in (1.22). Performing integrations by parts, the Dunford-Pettis theorem [19] yields a $\mathcal{B}_{\hat{\mu}}^{\hat{\mu}}(G, \mathcal{E})$-continuous mapping

$$
\mathcal{D}(G, \mathcal{E}) \rightarrow \mathcal{E}, \quad F \mapsto \int_{G} \mathbf{m E} F=\int_{G} \mathbf{E} \mathbf{D}_{\vec{r}_{j}}(\mathbf{m} F) .
$$

Then by Lemma 1.12 (v) (and Remark 1.13), the latter extends to the following continuous linear mapping:

$$
\widetilde{\int_{G} \mathbf{m}:}: \mathcal{B} \underline{\mu}(G, \mathcal{E}) \rightarrow \mathcal{E}
$$

that we refer to as an oscillatory integral. 
Our next aim is to prove that the oscillatory integral on $\mathcal{B}^{\mu}(G, \mathcal{E})$, does not depend on the choices made. So let $\mu, \underline{\mu}$ and $\hat{\mu}$ as in Definition 1.31. Fix $F \in$ $\mathcal{B} \underline{\mu}(G, \mathcal{E})$ and chose a sequence $\left\{F_{n}\right\}_{n \in \mathbb{N}}$ of elements of $\mathcal{D}(G, \mathcal{E})$ converging to $F$ for the topology of $\mathcal{B}^{\hat{\mu}}(G, \mathcal{E})$. By definition of the oscillatory integral and undoing the integrations by parts at the level of smooth compactly supported $\mathcal{E}$-valued functions, we first observe that by continuity, we have:

$$
\begin{aligned}
\widetilde{\int_{G} \mathbf{m} \mathbf{E}(F)} & =\widetilde{\int_{G} \mathbf{m} \mathbf{E}\left(\lim _{n \rightarrow \infty} F_{n}\right)} \\
& =\lim _{n \rightarrow \infty} \widetilde{\int_{G} \mathbf{m} \mathbf{E}}\left(F_{n}\right)=\lim _{n \rightarrow \infty} \int_{G} \mathbf{m}(g) \mathbf{E}(g) F_{n}(g) \mathrm{d}_{G}(g),
\end{aligned}
$$

where the first limit is in $\mathcal{B}^{\hat{\mu}}(G, \mathcal{E})$ and the last two are in $\mathcal{E}$. Then, the estimate of Proposition 1.29 immediately implies that the limit above is independent of the approximation sequence $\left\{F_{n}\right\}_{n \in \mathbb{N}}$ chosen. This shows that the oscillatory integral does not depend on the differential operators in $\mathbf{D}_{\vec{r}_{j}}$ used to define the extension (in the topology of $\left.\mathcal{B}^{\hat{\mu}}(G, \mathcal{E})\right)$ of the oscillatory integral from $\mathcal{D}(G, \mathcal{E})$ to $\mathcal{B} \underline{\mu}(G, \mathcal{E})$. Last, to see that the oscillatory integral mapping is also independent of the choice of the family of dominant weights $\hat{\mu}$ chosen, it suffices to remark that the approximation sequence constructed in the proof of Lemma 1.8 (viii) can be used for any family $\underline{\hat{\mu}}$ such that $\mu_{j} \prec \hat{\mu}_{j}$. Of course this will hold provided that we can always find dominant weights. This is certainly the case if there exists a weight dominating the constant weight 1 . Thus we have proved:

Proposition 1.32. Let $(G, S)$ be an admissible tempered pair, $\mathcal{E}$ be a complex Fréchet space, $\mu$ be tempered weights, $\mathbf{m} \in \mathcal{B}^{\mu}(G)$ and $\mu$ be a family of tempered weights. Assuming that there exists a tempered weight $\mu_{c}$ which dominates the constant weight 1 , then the oscillatory integral mapping

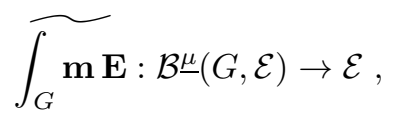

does not depend on the choice of the integers $\vec{r}_{j} \in \mathbb{N}^{N+1}$ and on the family of dominant weights $\underline{\hat{\mu}}$ given in Definition 1.31. Moreover, given $F \in \mathcal{B} \underline{\mu}(G, \mathcal{E})$, we have

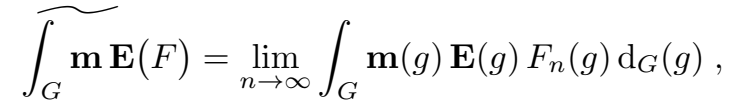

where $\left\{F_{n}\right\}_{n \in \mathbb{N}}$ is an arbitrary sequence in $\mathcal{D}(G, \mathcal{E})$, converging to $F$ in the topology of $\mathcal{B} \underline{\hat{\mu}}(G, \mathcal{E})$, for an arbitrary sequence of weights $\underline{\hat{\mu}}$, which dominates $\underline{\mu}$.

Remark 1.33. Note that Proposition 1.32 does not assert that the oscillatory integral on $\mathcal{B}^{\mu}(G, \mathcal{E})$ is the unique continuous extension of its restriction to $\mathcal{D}(G, \mathcal{E})$.

We observe that the existence of a tempered weight that dominates the constant weight 1 implies that every weight is dominated, which is crucial for the construction of the oscillatory integral, as observed above. This leads us to introduce the notion of tameness below. We will see that this property holds for negatively curved Kählerian groups, where we can simply use the modular weight (see Corollary 2.28 and Lemma 2.31). 
Definition 1.34. A tempered Lie group $G$, with associated diffeomorphism $\phi: G \rightarrow \mathfrak{g}^{\star}$ is called tame if there exist an Euclidean norm |.| on $\mathfrak{g}^{\star}$, a tempered weight $\mu_{\phi}$ and two positive constants $C, \rho$ such that

$$
C\left(1+|\phi|^{2}\right)^{\frac{\rho}{2}} \leq \mu_{\phi}
$$

Remark 1.35. Observe that when a tempered Lie group $G$ is tame, then there exists a tempered weight that dominates the constant weight 1 . Indeed, $1 \prec \mu_{\phi}$. This is the main reason for introducing this notion.

In the constant family case, i.e. for $\mathcal{B}^{\mu}(G, \mathcal{E})$, we can express the oscillatory integral as an absolutely convergent one for each semi-norm $\|\cdot\|_{j}$ :

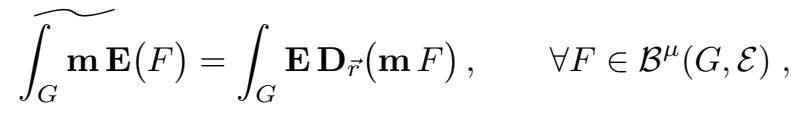

where the label $\vec{r} \in \mathbb{N}^{N+1}$ of the differential operator $\mathbf{D}_{\vec{r}}$ is given by Proposition 1.28 and does not depend on the index $j \in \mathbb{N}$ which labels the semi-norms defining the topology of $\mathcal{E}$ nor on the element $F \in \mathcal{B}^{\mu}(G, \mathcal{E})$. However, we cannot access to such a formula in the case of $\mathcal{B}=(G, \mathcal{E})$, since in this case the label $\vec{r} \in \mathbb{N}^{N+1}$ depends on the index $j \in \mathbb{N}$. However, and from Proposition 1.29, we have the following weaker statement:

Proposition 1.36. Let $(G, S)$ an admissible and tame tempered pair, $\mathcal{E}$ a Fréchet space, $\mu, \underline{\mu}$ be tempered weights and $\mathbf{m} \in \mathcal{B}^{\mu}(G)$. Then for every $j \in \mathbb{N}$, there exists $\vec{r}_{j} \in \overline{\mathbb{N}}^{N+1}$, such that for all $F \in \mathcal{B}^{\mu}(G, \mathcal{E})$, we have

$$
\widetilde{\int_{G} \mathbf{E}} \mathbf{E}(F)=\int_{G} \mathbf{E} \mathbf{D}_{\vec{r}_{j}}(\mathbf{m} F)
$$

where the right hand side is an absolutely convergent integral for the semi-norm $\|\cdot\|_{j}$.

We close this section with a natural result on the compatibility of the oscillatory integral with continuous linear maps between Fréchet spaces.

Lemma 1.37. Let $(G, S)$ be an admissible and tame tempered pair, $\left(\mathcal{E},\left\{\|\cdot\|_{j}\right\}\right)$ and $\left(\mathcal{F},\left\{\|\cdot\|_{j}^{\prime}\right\}\right)$ two Fréchet spaces and $T: \mathcal{E} \rightarrow \mathcal{F}$ a continuous linear map. Define the map $\hat{T}$ from $C(G, \mathcal{E})$ to $C(G, \mathcal{F})$, by setting

$$
(\hat{T} F)(g):=T(F(g)) .
$$

Then, for any family $\underline{\mu}$ of tempered weights, there exists another family of tempered weights $\underline{\nu}$, such that $\hat{T}$ is continuous from $\mathcal{B} \underline{\mu}(G, \mathcal{E})$ to $\mathcal{B} \underline{\underline{\nu}}(G, \mathcal{F})$. Moreover for any $\mathbf{m} \in \mathcal{B}^{\mu}(G)$, with $\mu$ another tempered weight, we have

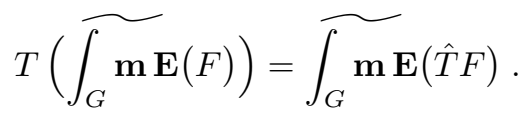

Proof. By continuity of $T$, for all $j \in \mathbb{N}$ there exist $l(j) \in \mathbb{N}$ and $C_{j}>0$, such that for all $a \in \mathcal{E}$, we have $\|T(a)\|_{j}^{\prime} \leq C_{j}\|a\|_{l(j)}$. This immediately implies the continuity of $\hat{T}$. Indeed, setting $\nu_{j}:=\mu_{l(j)}$, then for $F \in \mathcal{B} \underline{\nu}(G, \mathcal{E})$ and $j, k \in \mathbb{N}$, we 
have

$$
\begin{aligned}
\|\hat{T} F\|_{j, k, \underline{\nu}}^{\prime} & =\sup _{X \in \mathcal{U}_{k}(\mathfrak{g})} \sup _{g \in G} \frac{\|\widetilde{X} T(F(g))\|_{j}^{\prime}}{\mu_{l(j)}|X|_{k}}=\sup _{X \in \mathcal{U}_{k}(\mathfrak{g})} \sup _{g \in G} \frac{\|T(\tilde{X} F(g))\|_{j}^{\prime}}{\mu_{l(j)}|X|_{k}} \\
& \leq C_{j} \sup _{X \in \mathcal{U}_{k}(\mathfrak{g})} \sup _{g \in G} \frac{\|(\widetilde{X} F(g))\|_{l(j)}}{\mu_{l(j)}|X|_{k}}=C_{j}\|F\|_{l(j), k, \underline{\mu}} .
\end{aligned}
$$

Repeating the arguments for $\mathcal{B}^{\mu} \cdot \mu_{\phi}(G, \mathcal{E})\left(\mu_{\phi}\right.$ is the tempered weight associated to tameness) instead of $\mathcal{B} \underline{\mu}(G, \mathcal{E})$, we see that both sides of (1.26) define continuous linear maps from $\mathcal{B} \underline{\mu} \cdot \mu_{\phi}(G, \mathcal{E})$ to $\mathcal{F}$. Moreover, it is easy to see that they coincide on $\mathcal{D}(G, \mathcal{E})$ and thus they coincide on the closure of $\mathcal{D}(G, \mathcal{E})$ inside $\mathcal{B}^{\mu}{ }^{\mu} \mu_{\phi}(G, \mathcal{E})$, which contains $\mathcal{B} \underline{\mu}(G, \mathcal{E})$ by Lemma 1.12 (vi), as $\underline{\mu} . \mu_{\phi}$ dominates $\underline{\mu}$.

\subsection{A Fubini Theorem for semi-direct products}

The aim of this section is to prove a Fubini type result for the oscillatory integral on a semi-direct product of tempered pairs. We start with following observation:

Lemma 1.38. Let $\mathcal{E}$ be a Fréchet space, $G_{1}, G_{2}$ be two Lie groups with Lie algebras $\mathfrak{g}_{1}, \mathfrak{g}_{2}$ and $\mathbf{R} \in \operatorname{Hom}\left(G_{1}, \operatorname{Aut}\left(G_{2}\right)\right)$ be an extension homomorphism. Consider $\underline{\mu}$, a family of weights on the semi-direct product $G_{1} \ltimes_{\mathbf{R}} G_{2}$, with sub-multiplicative $\overline{\text { degree }}(\underline{L}, \underline{R})$. Set also $\underline{\mu}_{1}$ and $\underline{\mu}_{2}$ for its restrictions to the subgroups $G_{1}$ and $G_{2}$ and let $\mathfrak{d}_{1}$ be the restriction of the modular weight (cf. Definition 1.3) of $G_{1} \ltimes_{\mathbf{R}} G_{2}$ to $G_{1}$. Then the map

$$
\begin{aligned}
C^{\infty}\left(G_{1} \ltimes_{\mathbf{R}} G_{2}, \mathcal{E}\right) & \rightarrow C^{\infty}\left(G_{1}, C^{\infty}\left(G_{2}, \mathcal{E}\right)\right), \\
F & \mapsto \hat{F}:=\left[g_{1} \in G_{1} \mapsto\left[g_{2} \in G_{2} \mapsto F\left(g_{2} g_{1}\right)\right]\right],
\end{aligned}
$$

sends continuously $\mathcal{B} \underline{\mu}\left(G_{1} \ltimes_{\mathbf{R}} G_{2}, \mathcal{E}\right)$ to $\mathcal{B} \underline{\nu}\left(G_{1}, \mathcal{B} \underline{\underline{\lambda}}\left(G_{2}, \mathcal{E}\right)\right)$, where $\underline{\nu}:=\left\{\nu_{j, k}\right\}_{j, k \in \mathbb{N}}$ and $\underline{\lambda}:=\left\{\lambda_{j}\right\}_{j \in \mathbb{N}}$ with

$$
\nu_{j, k}:=\mu_{1, j}^{R_{j}} \mathfrak{d}_{1}^{k}, \quad \lambda_{j}:=\mu_{2, j}^{L_{j}} .
$$

Proof. First, observe that for $g \in G_{1} \ltimes_{\mathbf{R}} G_{2}$ with $g=g_{2} g_{1}, g_{1} \in G_{1}, g_{2} \in G_{2}$, $F \in C^{\infty}\left(G_{1} \ltimes_{\mathbf{R}} G_{2}\right)$ and for $X^{1} \in \mathfrak{g}_{1}, X^{2} \in \mathfrak{g}_{2}$, we have

$$
\widetilde{X^{1}}{ }_{. g} \hat{F}\left(g_{1}, g_{2}\right)=\widetilde{X^{1}}{ }_{. g} F(g), \quad \widetilde{X^{2}} \cdot g_{2} \hat{F}\left(g_{1}, g_{2}\right)=\widetilde{\mathbf{R}_{g_{1}^{-1}}\left(X^{2}\right)}{ }_{. g} F(g),
$$

where we use the same notation for the extension homomorphism and its derivative:

$$
\mathfrak{g}_{2} \rightarrow \mathfrak{g}_{2},\left.\quad X \mapsto \frac{d}{d t}\right|_{t=0} \mathbf{R}_{g_{1}}\left(e^{t X}\right), \quad g_{1} \in G_{1} .
$$

From this, it follows that the restriction of a weight on $G_{1} \ltimes_{\mathbf{R}} G_{2}$ to $G_{1}$ or $G_{2}$ is still a weight on $G_{1}$ or $G_{2}$. Indeed, given $\mu$ a weight on $G_{1} \ltimes_{\mathbf{R}} G_{2}$, call $\mu^{i}$, $i=1,2$, its restriction to the subgroup $G_{i}$ and given $X \in \mathcal{U}\left(\mathfrak{g}_{i}\right)$ call $X_{i}$ its image in $\mathcal{U}\left(\mathfrak{g}_{1} \ltimes \mathfrak{g}_{2}\right)$. Then, Equation (1.28) yields $\widetilde{X} \mu^{i}=\left(\widetilde{X}_{i} \mu\right)^{i}, i=1,2$, which together with $\left(\mu^{\vee}\right)^{i}=\left(\mu^{i}\right)^{\vee}$ (where $\mu^{\vee}(g):=\mu\left(g^{-1}\right)$ ) implies that the first condition of Definition 1.1 is satisfied. Sub-multiplicativity at the level of each subgroups $G_{i}$, $i=1,2$, follows from sub-multiplicativity at the level of $G_{1} \ltimes_{\mathbf{R}} G_{2}$ (with the same sub-multiplicativity degree). 
Moreover, (1.28) also implies that for $F \in \mathcal{B} \underline{\mu}\left(G_{1} \ltimes_{\mathbf{R}} G_{2}, \mathcal{E}\right), X^{1} \in \mathcal{U}_{k_{1}}\left(\mathfrak{g}_{1}\right)$, $X^{2} \in \mathcal{U}_{k_{2}}\left(\mathfrak{g}_{2}\right)$ and $k_{1}, k_{2}, j \in \mathbb{N}$, we have for $g_{2} g_{1} \in G_{1} \ltimes_{\mathbf{R}} G_{2}$ :

$$
\begin{aligned}
& \left.\left\|\widetilde{X^{1}}{ }_{. g_{1}} \widetilde{X^{2}}{ }_{. g_{2}} \hat{F}\left(g_{1}, g_{2}\right)\right\|_{j}=\|\left(\widetilde{X^{1}} \mathbf{R}_{g_{1}^{-1}\left(X^{2}\right.}\right) F\right)\left(g_{2} g_{1}\right) \|_{j} \\
& \leq C\left(k_{1}, k_{2}\right)\left|X^{1}\right|_{k_{1}}\left|X^{2}\right|_{k_{2}}\left|\mathbf{R}_{g_{1}^{-1}}\right|_{k_{2}} \sup _{Y \in \mathcal{U}_{k_{1}+k_{2}}\left(\mathfrak{g}_{1} \ltimes \mathfrak{g}_{2}\right)} \frac{\left\|\tilde{Y} F\left(g_{2} g_{1}\right)\right\|_{j}}{|Y|_{k_{1}+k_{2}}} \\
& \leq C\left(k_{1}, k_{2}\right)\left|X^{1}\right|_{k_{1}}\left|X^{2}\right|_{k_{2}}\left|\mathbf{R}_{g_{1}^{-1}}\right|_{k_{2}} \mu_{j}\left(g_{2} g_{1}\right)\|F\|_{j, k_{1}+k_{2}, \underline{\mu}} \\
& \leq C^{\prime}\left(k_{1}, k_{2}\right)\left|X^{1}\right|_{k_{1}}\left|X^{2}\right|_{k_{2}} \mathfrak{d}_{1}\left(g_{1}\right)^{k_{2}} \mu_{j}\left(g_{1}\right)^{R_{j}} \mu_{j}\left(g_{2}\right)^{L_{j}}\|F\|_{j, k_{1}+k_{2}, \underline{\mu}},
\end{aligned}
$$

by Lemma 1.14, since for $g_{1} \in G_{1}, \mathbf{R}_{g_{1}}$ coincides with the restriction of $\mathrm{Ad}_{g_{1}}$ to $\mathfrak{g}_{2}$. Thus, labeling by $\left(j, k_{2}\right) \in \mathbb{N}^{2}$ the semi-norms $\|\cdot\|_{j, k_{2}, \underline{\lambda}}$ of $\mathcal{B} \underline{\lambda}\left(G_{2}, \mathcal{E}\right)$, we finally get:

$$
\|\hat{F}\|_{\left(j, k_{2}\right), k_{1}, \underline{\nu}} \leq C^{\prime}\left(k_{1}, k_{2}\right)\|F\|_{j, k_{1}+k_{2}, \underline{\mu}},
$$

which completes the proof.

Now, assume that the groups $G_{1}$ and $G_{2}$ come from admissible tempered pairs $\left(G_{1}, S_{1}\right)$ and $\left(G_{2}, S_{2}\right)$. Parametrizing $g=g_{2} g_{1} \in G_{1} \ltimes_{\mathbf{R}} G_{2}$ with $g_{i} \in G_{i}, i=1,2$, we can then set

$$
S: G_{1} \ltimes_{\mathbf{R}} G_{2} \rightarrow \mathbb{R}, \quad g_{2} g_{1} \mapsto S_{1}\left(g_{1}\right)+S_{2}\left(g_{2}\right),
$$

and using the notation (1.17), we set accordingly

$$
\mathbf{E}\left(g_{2} g_{1}\right):=\exp \left\{i S\left(g_{2} g_{1}\right)\right\}=\mathbf{E}_{1}\left(g_{1}\right) \mathbf{E}_{2}\left(g_{2}\right) \text {. }
$$

For $g_{1} \in G_{1}$, we let $\left|\mathbf{R}_{g_{1}}\right|$ be the operator norm of $\mathbf{R}_{g_{1}} \in \operatorname{End}\left(\mathfrak{g}_{2}\right)$. Assume further that the map $G_{1} \rightarrow \mathbb{R}^{+}, g_{1} \mapsto\left|\mathbf{R}_{g_{1}}\right|$ is tempered. Then, by Lemma 1.5] and Lemma 1.21, we deduce that $\mathfrak{d}_{1}$, the restriction of the modular weight on $G_{1} \ltimes_{\mathbf{R}} G_{2}$ to $G_{1}$, is tempered too. Thus for $\mathbf{m} \in \mathcal{B}^{\mu}\left(G_{1} \ltimes_{\mathbf{R}} G_{2}\right)$ with $\mu$ a tempered weight, and with $\hat{\mathbf{m}}$ the associated function on $G_{1} \times G_{2}$ as constructed in (1.27), Lemma 1.38 shows that the map

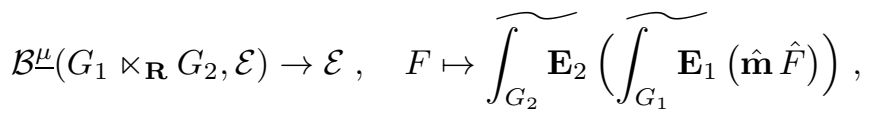

is well defined as a continuous linear map. Thus under these circumstances, this map could be used as a definition for the oscillatory integral on the semi-direct product $G_{1} \ltimes_{\mathbf{R}} G_{2}$. Moreover, when the pair $\left(G_{1} \ltimes_{\mathbf{R}} G_{2}, S\right)$ is also tempered and admissible and when the extension homomorphism preserves the Haar measure $\mathrm{d}_{G_{2}}$, then the map above coincides with the oscillatory integral on $G_{1} \ltimes_{\mathbf{R}} G_{2}$, as given in Definition 1.31. This is our Fubini-type result in the context of semi-direct product of tempered pairs:

Proposition 1.39. Within the context of Lemma 1.38, assume further that the groups $G_{1}$ and $G_{2}$ come from admissible and tame tempered pairs $\left(G_{1}, S_{1}\right)$ and $\left(G_{2}, S_{2}\right)$ and, with $S$ defined in (1.29), that $\left(G_{1} \ltimes_{\mathbf{R}} G_{2}, S\right)$ is admissible, tame and tempered too. Assume last that the extension homomorphism

$$
\mathbf{R} \in \operatorname{Hom}\left(G_{1}, \operatorname{Aut}\left(G_{2}\right)\right),
$$

preserves the Haar measure $\mathrm{d}_{G_{2}}$ and be such that the map $\left[g_{1} \mapsto\left|\mathbf{R}_{g_{1}}\right|\right]$ is tempered on $\left(G_{1}, S_{1}\right)$. Let also $\mu, \underline{\mu}$ be tempered weights on the semi-direct product $G_{1} \ltimes_{\mathbf{R}} G_{2}$. 
Then, for $F \in \mathcal{B} \underline{\mu}\left(G_{1} \ltimes_{\mathbf{R}} G_{2}, \mathcal{E}\right), \mathbf{m} \in \mathcal{B}^{\mu}\left(G_{1} \ltimes_{\mathbf{R}} G_{2}\right)$, with $\hat{F}$ and $\hat{\mathbf{m}}$ the associated functions on $G_{1} \times G_{2}$ as in (1.27), we have

$$
\int_{G_{1} \ltimes_{\mathbf{R}} G_{2}} \operatorname{Em}(F)=\widetilde{\int_{G_{2}} \mathbf{E}_{2}}\left(\widetilde{\left.\int_{G_{1}} \mathbf{E}_{1}(\hat{\mathbf{m}} \hat{F})\right) .}\right.
$$

Proof. Since the map $\left[g_{1} \mapsto\left|\mathbf{R}_{g_{1}}\right|\right]$ is tempered on $\left(G_{1}, S_{1}\right)$, by Lemma [1.5 and Lemma 1.21 one deduces that $\mathfrak{d}_{1}$, the restriction of the modular weight on $G_{1} \ltimes_{\mathbf{R}} G_{2}$ to $G_{1}$ is tempered on $\left(G_{1}, S_{1}\right)$. Thus by Lemma 1.38 the right handside of (1.30) is well defined as a continuous linear map from $\mathcal{B}^{\underline{\mu}} \cdot \mu_{\phi}\left(G_{1} \ltimes_{\mathbf{R}} G_{2}, \mathcal{E}\right)$ to $\mathcal{E}$ ( $\mu_{\phi}$ is the tempered weight on $G_{1} \ltimes_{\mathbf{R}} G_{2}$ associated with tameness). Note also that, by our assumptions, the pair $\left(G_{1} \ltimes_{\mathbf{R}} G_{2}, S\right)$ is tempered and admissible, the left hand side of (1.30) is also well defined as a continuous linear map from $\mathcal{B} \underline{\mu} \cdot \mu_{\phi}\left(G_{1} \ltimes_{\mathbf{R}} G_{2}, \mathcal{E}\right)$ to $\mathcal{E}$, too. Now, take $F \in \mathcal{D}\left(G_{1} \ltimes_{\mathbf{R}} G_{2}, \mathcal{E}\right)$ and associate to it $\hat{F} \in \mathcal{D}\left(G_{1}, \mathcal{D}\left(G_{2}, \mathcal{E}\right)\right)$ as in (1.27). By construction, we have

$$
\int_{G_{1} \ltimes_{\mathbf{R}} G_{2}} \mathbf{E m}(F)=\int_{G_{1} \ltimes_{\mathbf{R}} G_{2}} \mathbf{E}(g) \mathbf{m}(g) F(g) \mathrm{d}_{G_{1} \ltimes_{\mathbf{R}} G_{2}}(g) .
$$

Since the extension homomorphism $\mathbf{R}$ preserves $\mathrm{d}_{G_{2}}$, we have for $g_{1} \in G_{1}, g_{2} \in G_{2}$ :

$$
\mathrm{d}_{G_{1} \ltimes_{\mathbf{R}} G_{2}}\left(g_{2} g_{1}\right)=\mathrm{d}_{G_{1}}\left(g_{1}\right) \mathrm{d}_{G_{2}}\left(g_{2}\right),
$$

which, by the ordinary Fubini Theorem, implies that

$$
\begin{aligned}
\int_{G_{1} \ltimes_{\mathbf{R}} G_{2}} \mathbf{E m}(F) & =\int_{G_{2}} \mathbf{E}_{2}\left(g_{2}\right)\left(\int_{G_{1}} \mathbf{E}_{1}\left(g_{1}\right) \mathbf{m}\left(g_{2} g_{1}\right) F\left(g_{2} g_{1}\right) \mathrm{d}_{G_{1}}\left(g_{1}\right)\right) \mathrm{d}_{G_{2}}\left(g_{2}\right) \\
& =\int_{G_{2}} \mathbf{E}_{2}\left(\int_{G_{1}} \mathbf{E}_{1}(\hat{\mathbf{m}} \hat{F})\right) .
\end{aligned}
$$

Thus, both sides of (1.30) are continuous linear maps from $\mathcal{B} \underline{\mu} \cdot \mu_{\phi}\left(G_{1} \ltimes_{\mathbf{R}} G_{2}, \mathcal{E}\right)$ to $\mathcal{E}$ and coincide on $\mathcal{D}\left(G_{1} \ltimes_{\mathbf{R}} G_{2}, \mathcal{E}\right)$. Therefore, these maps coincide on the closure of $\mathcal{D}\left(G_{1} \ltimes_{\mathbf{R}} G_{2}, \mathcal{E}\right)$ inside $\mathcal{B}^{\mu} \cdot \mu_{\phi}\left(G_{1} \ltimes_{\mathbf{R}} G_{2}, \mathcal{E}\right)$. One concludes using Lemma 1.12 (vi), which shows that the latter closure contains $\mathcal{B}^{\mu}\left(G_{1} \ltimes_{\mathbf{R}} G_{2}, \mathcal{E}\right)$.

\subsection{A Schwartz space for tempered pairs}

In this section, we introduce a Schwartz type functions space, out of an admissible tempered pair $(G, S)$ and prove that it is Fréchet and nuclear. Our notion of Schwartz space is of course closely related, if not in many cases equivalent, to other notions of Schwartz space on Lie groups, but the point here is that it is formulated in terms of the phase function $S$ only. This is this formulation that allows to immediately implement the compatibility with our notion of oscillatory integral.

Definition 1.40. Let $(G, S)$ be a tempered pair. For all $X \in \mathcal{U}(\mathfrak{g})$, we let $\alpha_{X}:=\mathbf{E}^{-1} \widetilde{X} \mathbf{E} \in C^{\infty}(G)$, where $\mathbf{E}$ is defined in (1.17). Then we set

$$
\mathcal{S}^{S}(G):=\left\{f \in C^{\infty}(G): \forall X, Y \in \mathcal{U}(\mathfrak{g}), \forall n \in \mathbb{N}, \sup _{x \in G}\left|\alpha_{X}^{n}(x)(\widetilde{Y} f)(x)\right|<\infty\right\} .
$$

We first prove that this space is isomorphic to the ordinary Schwartz space of the Euclidean space $\mathfrak{g}^{\star}$. 
Lemma 1.41. Let $\phi: G \rightarrow \mathfrak{g}^{\star}$ be the diffeomorphism underlying Definition 1.22, associated to an admissible tempered pair $(G, S)$. Fixing an Euclidean structure on $\mathfrak{g}^{\star}$, denote by $\mathcal{S}\left(\mathfrak{g}^{\star}\right)$ the ordinary Schwartz space of $\mathfrak{g}^{\star}$. Then, $\mathcal{S}^{S}(G)$ coincides with

$$
\mathcal{S}^{\phi}(G):=\left\{f \in C^{\infty}(G): f \circ \phi^{-1} \in \mathcal{S}\left(\mathfrak{g}^{\star}\right)\right\} .
$$

In particular, endowed with the transported topology, $\mathcal{S}^{S}(G)$ is a nuclear Fréchet space. If moreover the pair $(G, S)$ is tame, then the transported topology is equivalent to the one associated with the semi-norms

$$
\|\cdot\|_{k, n}: f \in \mathcal{S}^{S}(G, \mathcal{E}) \mapsto \sup _{X \in \mathcal{U}_{k}(\mathfrak{g})} \sup _{x \in G}\left\{\frac{\mu_{\phi}(x)^{n}|\tilde{X} f(x)|}{|X|_{k}}\right\}, \quad k, n \in \mathbb{N},
$$

Proof. Recall that $f \in \mathcal{S}^{\phi}(G)$ if and only if for all $\alpha, \beta \in \mathbb{N}^{\operatorname{dim}(G)}$, we have

$$
\sup _{\xi \in \mathfrak{g}^{\star}}\left|\xi^{\alpha} \partial^{\beta}\left(f \circ \phi^{-1}\right)(\xi)\right|<\infty
$$

while $f \in \mathcal{S}^{S}(G)$ if and only if for all $X, Y \in \mathcal{U}(\mathfrak{g})$ and all $n \in \mathbb{N}$

$$
\sup _{x \in G}\left|\alpha_{X}^{n}(x)(\tilde{Y} f)(x)\right|<\infty \text {. }
$$

Fix $\left\{X_{j}\right\}_{j=1}^{\operatorname{dim}(G)}$ a basis of $\mathfrak{g}$ and let $\left\{\xi_{j}\right\}_{j=1}^{\operatorname{dim}(G)}$ be the dual basis on $\mathfrak{g}^{\star}$. From the same methods as in Lemma 1.21 one can construct an invertible matrix $M(\xi)$ which is tempered with tempered inverse and which is such that in the $\phi$-coordinates

$$
\widetilde{X}_{j}=\sum_{i=1}^{\operatorname{dim}(G)} M(\xi)_{j, i} \partial_{\xi_{i}}
$$

Since by Remark 1.23 $S$ is tempered, for all $X \in \mathcal{U}(\mathfrak{g})$, the associated multiplier $\alpha_{X}=\mathbf{E}^{-1} \tilde{X} \mathbf{E}$ in $\phi$-coordinates is bounded by a polynomial function on $\mathfrak{g}^{\star}$. Last, since the pair $(G, S)$ is admissible, associated to the vector space decomposition $\mathfrak{g}=\bigoplus_{k=0}^{N} V_{k}$, there exist elements $X_{k} \in \mathcal{U}\left(V_{k}\right)$ and constants $C_{k}, \rho_{k}>0$ such that with $\alpha_{k}=\mathbf{E}^{-1} \widetilde{X_{k}} \mathbf{E}$ and with $|\cdot|_{k}$ the Euclidean norm on $V_{k}$, we have

$$
\left|x_{n}\right|_{n} \leq\left(C_{n}^{-1}\left|\alpha_{n}\right|-1\right)^{1 / \rho_{n}}
$$

Summing up over $k=1, \ldots, N$ gives the existence of $C, \rho>0$, such that

$$
|\xi| \leq C\left(1+\sum_{k=0}^{N}\left|\alpha_{k}\left(\phi^{-1}(\xi)\right)\right|\right)^{\rho}
$$

Putting these three facts together gives the equality between the two sets of functions on $G$ and the equivalence of the topologies associated with the semi-norms (1.31) and (1.32).

Last, assume that the pair $(G, S)$ is also tame, with associated weight $\mu_{\phi}$. Then there exist constants $C_{1}, C_{2}, \rho_{1}, \rho_{2}$ such that, with $\phi: G \rightarrow \mathfrak{g}^{\star}$ the diffeomorphism underlying Definition 1.22 , we have

$$
C_{1}\left(1+|\phi|^{2}\right)^{\rho_{1} / 2} \leq \mu_{\phi} \leq C_{2}\left(1+|\phi|^{2}\right)^{\rho_{2} / 2} .
$$

which is enough to prove the last claim. 
More generally, when $\mathcal{E}$ is a complex Fréchet space with topology underlying a countable set of semi-norms $\left\{\|\cdot\|_{j}\right\}_{j \in \mathbb{N}}$, we define the $\mathcal{E}$-valued Schwartz space associated to an admissible tempered and tame pair $(G, S)$ as the Fréchet space completion of $\mathcal{D}(G, \mathcal{E})$ for the topology underlying the family of semi-norms:

$$
\|f\|_{j, k, n}:=\sup _{X \in \mathcal{U}_{k}(\mathfrak{g})} \sup _{x \in G}\left\{\frac{\mu_{\phi}(x)^{n}\|\widetilde{X} f(x)\|_{j}}{|X|_{k}}\right\}, \quad j, k, n \in \mathbb{N},
$$

Note that by nuclearity of $\mathcal{S}^{S}(G)$, we have $\mathcal{S}^{S}(G, \mathcal{E})=\mathcal{S}^{S}(G) \hat{\otimes} \mathcal{E}$ (for any completed tensor product).

\subsection{Bilinear mappings from the oscillatory integral}

We now present several results which establish most of the analytical properties we will need to construct our universal deformation formula for actions of Kählerian groups on Fréchet algebras. In all that follows, when considering a Fréchet algebra $\mathcal{A}$ with topology underlying a countable set of semi-norms $\left\{\|\cdot\|_{j}\right\}_{j \in \mathbb{N}}$, we will always assume the latter to be sub-multiplicative, i.e.

$$
\|a b\|_{j} \leq\|a\|_{j}\|b\|_{j}, \quad \forall a, b \in \mathcal{A}, \quad \forall j \in \mathbb{N} .
$$

We start with a crucial fact. Its proof being very similar to those of Lemma 1.15] we omit it.

Lemma 1.42. Let $\mathcal{A}$ be a Fréchet algebra and let $\underline{\mu}_{1}, \underline{\mu}_{2}$ be two families of weights on $G$ with sub-multiplicative degree respectively denoted by $\left(\underline{L}_{1}, \underline{R}_{1}\right)$ and $\left(\underline{L}_{2}, \underline{R}_{2}\right)$. Then the bilinear mapping

$$
\begin{aligned}
& \mathcal{R} \otimes \mathcal{R}: C^{\infty}(G, \mathcal{A}) \times C^{\infty}(G, \mathcal{A}) \rightarrow C^{\infty}\left(G \times G, C^{\infty}(G, \mathcal{A})\right), \\
& \left(F_{1}, F_{2}\right) \mapsto\left[(x, y) \in G \times G \mapsto\left(R_{x}^{\star} F_{1}\right)\left(R_{y}^{\star} F_{2}\right):=\left[g \in G \mapsto F_{1}(g x) F_{2}(g y)\right] \in \mathcal{A}\right],
\end{aligned}
$$

is continuous from $\mathcal{B}^{\underline{\mu}}(G, \mathcal{A}) \times \mathcal{B}^{\underline{\mu}} \underline{\underline{\mu}}_{2}(G, \mathcal{A})$ to $\mathcal{B} \underline{\underline{\nu}}(G \times G, \mathcal{B} \underline{\lambda}(G, \mathcal{A}))$, where the families $\underline{\nu}:=\left\{\nu_{j, k}\right\}_{j, k \in \mathbb{N}}$ and $\underline{\lambda}:=\left\{\lambda_{j}\right\}_{j \in \mathbb{N}}$ are given by

$$
\nu_{j, k}:=\left(\mu_{1, j} \otimes \mu_{2, j}\right)^{\max \left(R_{1, j}, R_{2, j}\right)} \mathfrak{d}_{G \times G}^{k}, \quad \lambda_{j}:=\mu_{1, j}^{L_{1, j}} \mu_{2, j}^{L_{2, j}} .
$$

More precisely, labeling by $(j, k) \in \mathbb{N}^{2}$ the semi-norm $\|\cdot\|_{j, k, \underline{\lambda}}$ of $\mathcal{B} \underline{\lambda}(G, \mathcal{A})$, for all $\left(j, k, k^{\prime}\right)$ in $\mathbb{N}^{3}$, there exists $C>0$ such that for all $F_{1} \in \mathcal{B}^{\mu_{1}}(G, \mathcal{A}), F_{2} \in \mathcal{B} \underline{\mu}_{2}(G, \mathcal{A})$, we have

$$
\left\|\mathcal{R} \otimes \mathcal{R}\left(F_{1}, F_{2}\right)\right\|_{(j, k), k^{\prime}, \underline{\nu}} \leq C\left\|F_{1}\right\|_{j, k+k^{\prime}, \underline{\mu}_{1}}\left\|F_{2}\right\|_{j, k+k^{\prime}, \underline{\mu}_{2}} .
$$

TheOrem 1.43. Let $(G \times G, S)$ be an admissible and tame tempered pair. Let also $\mathbf{m} \in \mathcal{B}^{\mu}(G \times G)$ for some tempered weight $\mu$ on $G \times G$ and let $\underline{\mu}_{1}, \underline{\mu}_{2}$ be two families of weights on $G$ with sub-multiplicative degree respectively denoted by $\left(\underline{L}_{1}, \underline{R}_{1}\right)$ and $\left(\underline{L}_{2}, \underline{R}_{2}\right)$, such that the family of weights $\underline{\mu}_{1} \otimes \underline{\mu}_{2}$ is tempered on $G \times G$. Then, for any Fréchet algebra $\mathcal{A}$, the oscillatory integral

$$
\star_{S}:=\left[\left(F_{1}, F_{2}\right) \mapsto \int_{G \times G} \mathbf{m E} \circ \mathcal{R} \otimes \mathcal{R}\left(F_{1}, F_{2}\right)\right],
$$

defines a continuous bilinear map from $\mathcal{B}^{\underline{\mu}} \underline{1}_{1}(G, \mathcal{A}) \times \mathcal{B}^{\underline{\mu}} \underline{-}_{2}(G, \mathcal{A})$ to $\mathcal{B} \underline{\lambda}(G, \mathcal{A})$, where the family $\underline{\lambda}:=\left\{\lambda_{j}\right\}_{j \in \mathbb{N}}$, is given by

$$
\lambda_{j}:=\mu_{1, j}^{L_{1, j}} \mu_{2, j}^{L_{2, j}} .
$$


More precisely, for any $(j, k) \in \mathbb{N}^{2}$ there exist $C>0$ and $l \in \mathbb{N}$ such that for any $F_{1} \in \mathcal{B}^{\underline{\mu}}{ }_{1}(G, \mathcal{A})$ and $F_{2} \in \mathcal{B}^{\underline{\mu}}{ }_{2}(G, \mathcal{A})$, we have

$$
\left\|F_{1} \star_{S} F_{2}\right\|_{j, k, \underline{\lambda}} \leq C\left\|F_{1}\right\|_{j, l, \underline{\mu}_{1}}\left\|F_{2}\right\|_{j, l, \underline{\mu}_{2}} .
$$

In particular, one has a continuous bilinear product (not necessarily associative!):

$$
\star_{S}: \mathcal{B}(G, \mathcal{A}) \times \mathcal{B}(G, \mathcal{A}) \rightarrow \mathcal{B}(G, \mathcal{A}) .
$$

Proof. By Lemma 1.42, the map

$$
\mathcal{R} \otimes \mathcal{R}: \mathcal{B}^{\underline{\mu}}(G, \mathcal{A}) \times \mathcal{B}^{\underline{\mu}}{ }^{2}(G, \mathcal{A}) \rightarrow \mathcal{B}^{\underline{\nu}}\left(G \times G, \mathcal{B}^{\underline{\lambda}}(G, \mathcal{A})\right),
$$

where $\nu_{j, k}=\left(\mu_{1, j} \otimes \mu_{2, j}\right)^{\max \left(R_{1, j}, R_{2, j}\right)} \mathfrak{d}_{G \times G}^{k}$ and $\lambda_{j}=\mu_{1, j}^{L_{1, j}} \mu_{2, j}^{L_{2, j}}$, is a continuous bilinear mapping. By tameness, the family of tempered weights $\mu . \underline{\nu}$ is dominated. Hence the oscillatory integral composed with the map $\mathcal{R} \otimes \mathcal{R}$ is well defined as a continuous bilinear mapping from $\mathcal{B}^{\underline{\mu}} \underline{1}_{1}(G, \mathcal{A}) \times \mathcal{B}^{\underline{\mu}} \underline{\mu}_{2}(G, \mathcal{A})$ to $\mathcal{B} \underline{\lambda}(G, \mathcal{A})$. The precise estimate follows by putting together Proposition 1.29, Lemma 1.42 and the continuity relation $\|\cdot\|_{j, k, \underline{\hat{\mu}}} \leq C\|\cdot\|_{j, k, \underline{\mu}}$, for any family of weights $\underline{\hat{\mu}}$ that dominates another family $\underline{\mu}$.

We now discuss some issues regarding associativity of the bilinear mapping ${ }_{S}$. To this aim, we need to show how to compute the product $F_{1} \star_{S} F_{2}$ as the limit of a double sequence of products of smooth compactly supported functions.

Lemma 1.44. Within the context of Theorem 1.43, for $F_{1} \in \mathcal{B}^{\underline{\mu}}(G, \mathcal{A})$ and $F_{2} \in \mathcal{B}^{\mu_{2}}(G, \mathcal{A})$, we let $\left\{F_{1, n}\right\},\left\{F_{2, n}\right\}$ be two sequences in $\mathcal{D}(G, \mathcal{A})$ converging respectively to $F_{1}$ and $F_{2}$ for the topologies of $\mathcal{B} \underline{\hat{\mu}}_{1}(G, \mathcal{A})$ and $\mathcal{B}_{\underline{\mu}_{2}}(G, \mathcal{A})$ where $\underline{\hat{\mu}}_{1} \succ \underline{\mu}_{1}, \underline{\hat{\mu}}_{2} \succ \underline{\mu}_{2}$ are such that $\underline{\hat{\mu}}_{1} \otimes \underline{\hat{\mu}}_{2}$ is tempered on $G \times G$. Setting then $\lambda_{j}:=\mu_{1, j}^{L_{1, j}} \mu_{2, j}^{L_{2, j}}$, we have the equalities in $\mathcal{B}^{\lambda}(G, \mathcal{A})$ :

$$
F_{1} \star_{S} F_{2}=\lim _{n_{1} \rightarrow \infty} \lim _{n_{2} \rightarrow \infty} F_{1, n_{1}} \star_{S} F_{2, n_{2}}=\lim _{n_{2} \rightarrow \infty} \lim _{n_{1} \rightarrow \infty} F_{1, n_{1}} \star_{S} F_{2, n_{2}} .
$$

Proof. Note that the family $\underline{\hat{\nu}}:=\left\{\left(\hat{\mu}_{1, j} \otimes \hat{\mu}_{2, j}\right)^{\max \left(R_{1, j}, R_{2, j}\right)} \mathfrak{d}_{G \times G}^{k}\right\}_{(j, k)}$ is tempered (by assumption) and dominates the (tempered by assumption) family $\underline{\nu}:=\left\{\left(\mu_{1, j} \otimes \mu_{2, j}\right)^{\max \left(R_{1, j}, R_{2, j}\right)} \mathfrak{d}_{G \times G}^{k}\right\}_{(j, k)}$ and consequently (see Remark 1.13), we may view $\mathcal{R} \otimes \mathcal{R}\left(F_{1}, F_{2}\right)$ as an element of $\mathcal{B} \underline{\hat{\nu}}(G \times G, \mathcal{B} \underline{\lambda}(G, \mathcal{A}))$, with $\underline{\lambda}:=$ $\left\{\mu_{1, j}^{L_{1, j}} \mu_{2, j}^{L_{2, j}}\right\}_{j \in \mathbb{N}}$. By the estimate of Theorem 1.43 we know that for all $j, k \in \mathbb{N}$, there exists $l \in \mathbb{N}$ such that

$$
\left\|F_{1} \star_{S} F_{2}\right\|_{j, k, \underline{\lambda}} \leq C(k, j)\left\|F_{1}\right\|_{j, l, \underline{\mu}_{1}}\left\|F_{2}\right\|_{j, l, \underline{\mu}_{2}} .
$$

We then write

$$
\begin{aligned}
& \left\|F_{1} \star_{S} F_{2}-F_{1, n_{1}} \star_{S} F_{2, n_{2}}\right\|_{j, k, \underline{\lambda}} \\
& \leq\left\|\left(F_{1}-F_{1, n_{1}}\right) \star_{S} F_{2}\right\|_{j, k, \underline{\lambda}}+\left\|F_{1, n_{1}} \star_{S}\left(F_{2}-F_{2, n_{2}}\right)\right\|_{j, k, \underline{\lambda}} \\
& \leq C(k, j)\left(\left\|F_{1}-F_{1, n_{1}}\right\|_{j, l, \underline{\hat{\mu}}_{1}}\left\|F_{2}\right\|_{j, l, \underline{\underline{\mu}}_{2}}+\left\|F_{1, n_{1}}\right\|_{j, l, \underline{\hat{\mu}}_{1}}\left\|F_{2}-F_{2, n_{2}}\right\|_{j, l, \underline{\underline{\mu}}_{2}}\right) \\
& \leq C^{\prime}(k, j)\left(\left\|F_{1}-F_{1, n_{1}}\right\|_{j, l, \underline{\hat{\mu}}_{1}}\left\|F_{2}\right\|_{j, l, \underline{\mu}_{2}}+\left\|F_{1, n_{1}}\right\|_{j, l, \underline{\hat{\mu}}_{1}}\left\|F_{2}-F_{2, n_{2}}\right\|_{j, l, \underline{\hat{\mu}}_{2}}\right),
\end{aligned}
$$

where in the last inequality we used Remark 1.13 which shows that the semi-norm $\|\cdot\|_{j, k, \underline{\underline{\mu}}_{2}}$ is dominated by $\|\cdot\|_{j, k, \underline{\mu}_{2}}$. The latter remark also shows that the numerical sequence $\left\{\left\|F_{1, n_{1}}\right\|_{j, l, \underline{\mu}_{1}}\right\}_{n_{1} \in \mathbb{N}}$ is bounded since

$$
\left\|F_{1, n_{1}}\right\|_{j, l, \underline{\hat{\mu}}_{1}} \leq\left\|F_{1, n_{1}}-F_{1}\right\|_{j, l, \underline{\hat{\mu}}_{1}}+\left\|F_{1}\right\|_{j, l, \underline{\underline{\mu}}_{1}} \leq\left\|F_{1, n_{1}}-F_{1}\right\|_{j, l, \underline{\mu}_{1}}+C\left\|F_{1}\right\|_{j, l, \underline{\mu}_{1}} .
$$


This completes the proof.

REMARK 1.45. In other words, within the setting of Lemma 1.44, we have in $\mathcal{B}^{\lambda}(G, \mathcal{A})$ :

$$
F \star_{S} F^{\prime}=\lim _{m, n \rightarrow \infty} \int_{G \times G} \mathbf{E}\left(x, x^{\prime}\right) \mathbf{m}\left(x, x^{\prime}\right) R_{x}^{\star}\left(F_{m}\right) R_{x^{\prime}}^{\star}\left(F_{n}^{\prime}\right) \mathrm{d}_{G}(x) \mathrm{d}_{G}\left(x^{\prime}\right),
$$

for suitable approximation sequences $\left\{F_{n}\right\},\left\{F_{n}^{\prime}\right\} \subset \mathcal{D}(G, \mathcal{A})$.

DeFinition 1.46. Within the context of Theorem 1.43, we say that the product $\star_{S}$, given in (1.33), is weakly associative when for all $\psi_{1}, \psi_{2}, \psi_{3} \in \mathcal{D}(G, \mathcal{A})$, one has $\left(\psi_{1} \star_{S} \psi_{2}\right) \star_{S} \psi_{3}=\psi_{1} \star_{S}\left(\psi_{2} \star_{S} \psi_{3}\right)$ in $\mathcal{B}(G, \mathcal{A})$.

Proposition 1.47. Within the context of Theorem 1.43, weak associativity implies strong associativity in the sense that, when weakly associative, for every further family of weights $\underline{\mu}_{3}$ on $G$ with sub-multiplicative degree denoted by $\left(\underline{L}_{3}, \underline{R}_{3}\right)$ such that $\underline{\mu}_{2} \otimes \underline{\mu}_{3}$ is tempered on $G \times G$. Then, for every element $\left(F_{1}, F_{2}, F_{3}\right) \in$ $\mathcal{B} \underline{\mu}_{1}(G, \mathcal{A}) \times \mathcal{B} \underline{\mu}_{2}(G, \mathcal{A}) \times \mathcal{B} \underline{\mu}_{3}(G, \mathcal{A})$, one has the equality $\left(F_{1} \star_{S} F_{2}\right) \star_{S} F_{3}=F_{1} \star_{S}$ $\left(F_{2} \star_{S} F_{3}\right)$ in $\mathcal{B} \underline{\lambda}(G, \mathcal{A})$ for $\underline{\lambda}=\left\{\mu_{1, j}^{L_{1, j}^{2}} \mu_{2, j} L_{2, j}^{2} \mu_{3, j} L_{3, j}^{2}\right\}_{j \in \mathbb{N}}$.

Proof. Let $\mu_{\phi}$ be a tempered weight on $G \times G$ which dominates the constant weight 1 (it exists by assumption of tameness.) Consider the element $\nu_{\phi} \in C^{\infty}(G)$ defined by $\nu_{\phi}(g):=\mu_{\phi}(g, e)$. The latter is then (by a direct application of Lemma 1.5) a weight on $G$ that dominates 1 . Moreover, it is easy to see that $\nu_{\phi} \otimes \nu_{\phi}$ is tempered on $G \times G$. Hence, all the family of weights $\underline{\mu}_{1}, \underline{\mu}_{2}$ and $\underline{\mu}_{3}$ are dominated e.g. by $\underline{\hat{\mu}}_{1}:=\nu_{\phi} \cdot \underline{\mu}_{1}, \underline{\hat{\mu}}_{2}:=\nu_{\phi} \cdot \underline{\mu}_{2}$ and $\underline{\hat{\mu}}_{3}:=\bar{\nu}_{\phi} \cdot \underline{\mu}_{3}$ and $\underline{\hat{\mu}}_{1} \otimes \underline{\hat{\mu}}_{2}$ and $\underline{\hat{\mu}}_{2} \otimes \underline{\hat{\mu}}_{3}$ are tempered on $G \times G$. The assumptions of Lemma $1 . \overline{44}$ are therefore satisfied. Let us consider sequences of smooth compactly supported elements $\left\{\Phi_{1, n}\right\}_{n \in \mathbb{N}},\left\{\Phi_{2, n}\right\}_{n \in \mathbb{N}}$ and $\left\{\Phi_{3, n}\right\}_{n \in \mathbb{N}}$ that converge to the elements $F_{1}, F_{2}$ and $F_{3}$ respectively in $\mathcal{B} \underline{\hat{\mu}}_{1}(G, \mathcal{A}), \mathcal{B}^{\hat{\mu}_{2}}(G, \mathcal{A})$ and $\mathcal{B}^{\hat{\mu}_{3}}(G, \mathcal{A})$. Using separate continuity of $\star_{S}$ and Lemma 1.44, we observe the following equality:

$$
\lim _{n_{1} \rightarrow \infty}\left(\lim _{n_{2} \rightarrow \infty}\left(\lim _{n_{3} \rightarrow \infty}\left(\Phi_{1, n_{1}} \star_{S} \Phi_{2, n_{2}}\right) \star_{S} \Phi_{3, n_{3}}\right)\right)=\left(F_{1} \star_{S} F_{2}\right) \star_{S} F_{3},
$$

in $\mathcal{B} \underline{\lambda}(G, \mathcal{A})$ for $\underline{\lambda}=\left\{\mu_{1, j}^{L_{1, j}^{2}} \mu_{2, j}{ }_{2, j}^{2} \mu_{3, j}{ }^{2}{ }_{3, j}\right\}$. One then concludes using weak associativity and the commutativity of the limits, as shown in Lemma 1.44

In section 1.5. we have seen how to associate in a canonical way a Schwartz type functions space to a tempered, admissible and tame pair. Hence, starting with such a pair $(G \times G, S)$, we get a Schwartz space on $G \times G$. But we can also define a one-variable Schwartz space using the continuity of the partial evaluation maps:

Definition 1.48. Let $(G \times G, S)$ be a tempered admissible and tame pair and $\mathcal{A}$ be a Fréchet algebra. We define the $\mathcal{A}$-valued Schwartz space on $G$ associated to $S$ by

$$
\mathcal{S}^{S}(G, \mathcal{A}):=\left\{[g \in G \mapsto f(g, e)], f \in \mathcal{S}^{S}(G \times G, \mathcal{A})\right\} .
$$

We endow the latter with the topology induced by the semi-norms:

$$
\|f\|_{j, k, n}:=\sup _{X \in \mathcal{U}_{k}(\mathfrak{g})} \sup _{x \in G}\left\{\frac{\mu_{\phi, 1}(x)^{n}\|\tilde{X} f(x)\|_{j}}{|X|_{k}}\right\}, \quad j, k, n \in \mathbb{N},
$$


with $\mu_{\phi, 1}(x):=\mu_{\phi}(x, e)$ and $\mu_{\phi}$ is the tempered weight on $G \times G$ associated with the tameness (Definition 1.34).

The next Lemma shows that the right action on the space of $\mathcal{A}$-valued Schwartz functions, leads us to a $\mathcal{B}$-type space for family of weights too.

Lemma 1.49. Let $(G \times G, S)$ be a tame and admissible tempered pair, $\mathcal{A}$ be a Fréchet algebra and $\underline{\mu}$ be a family of weights on $G$ with sub-multiplicative degree $(\underline{L}, \underline{R})$, which is bounded by a power of $\mu_{\phi, 1}$ (see Definition 1.48). Then, there exists a sequence $\left\{M_{n}\right\}_{n \in \mathbb{N}}$ of integers, such that for all elements $F \in \mathcal{B} \underline{\mu}(G, \mathcal{A})$ and $\varphi \in \mathcal{S}^{S}(G, \mathcal{A})$, the element $(\mathcal{R} \otimes \mathcal{R})(F, \varphi)$ (defined in Lemma 1.42) belongs to $\mathcal{B} \underline{\nu}\left(G \times G, \mathcal{S}^{S}(G, \mathcal{A})\right)$ where $\underline{\nu}:=\left\{\nu_{j, k, n}\right\}_{j, k, n \in \mathbb{N}}$ with

$$
\nu_{j, k, n}:=\left(\mu_{j}^{R_{j}} \otimes \mu_{\phi, 1}^{\vee M_{n}}\right) \mathfrak{d}_{G \times G}^{2 k}, \quad j, k, n \in \mathbb{N} .
$$

Proof. Denote by $\|\cdot\|_{j, k, n},(j, k, n) \in \mathbb{N}^{3}$, the semi-norms (1.34) of $\mathcal{S}^{S}(G, \mathcal{A})$. Then, the semi-norms of an element

$$
\Phi=[(x, y) \in G \times G \mapsto[g \in G \mapsto \Phi(x, y ; g) \in \mathcal{A}]] \in \mathcal{B}^{\underline{\nu}}\left(G \times G, \mathcal{S}^{S}(G, \mathcal{A})\right),
$$

are given by

$$
\begin{aligned}
& \|\Phi\|_{(j, k, n),\left(k_{1}, k_{2}\right), \underline{\nu}}= \\
& \sup _{x, y, g \in G} \sup _{X \in \mathcal{U}_{k}(\mathfrak{g})\left(Y_{1}, Y_{2}\right) \in \mathcal{U}_{k_{1}}(\mathfrak{g}) \times \mathcal{U}_{k_{2}}(\mathfrak{g})} \frac{\mu_{\phi, 1}(g)^{n}\left\|\widetilde{X}_{g}\left(\widetilde{Y}_{1} \otimes \tilde{Y}_{2}\right)_{(x, y)} \Phi(x, y ; g)\right\|_{j}}{\nu_{j, k, n}(x, y)} .
\end{aligned}
$$

Using Sweedler's notation (0.2), we have for $X \in \mathcal{U}_{k}(\mathfrak{g}), Y_{1} \in \mathcal{U}_{k_{1}}(\mathfrak{g}), Y_{2} \in \mathcal{U}_{k_{2}}(\mathfrak{g})$ :

$$
\begin{aligned}
& \tilde{X}_{g} \cdot\left(\left(\widetilde{Y}_{1} \otimes \tilde{Y}_{2}\right)_{(x, y)}\left(R_{x}^{\star} F(g) R_{y}^{\star} \varphi(g)\right)\right)= \\
& \sum_{(X)}\left(\left(\widetilde{\operatorname{Ad}_{x^{-1}} X_{1}}\right) \widetilde{Y}_{1} F\right)(g x)\left(\left(\widetilde{\operatorname{Ad}_{y^{-1}} X_{2}}\right) \widetilde{Y}_{2} \varphi\right)(g y),
\end{aligned}
$$

which yields the following estimation for arbitrary $N \in \mathbb{N}$ :

$$
\begin{aligned}
& \left\|\widetilde{X}_{g} \cdot\left(\left(\widetilde{Y}_{1} \otimes \widetilde{Y}_{2}\right)_{(x, y)} \cdot\left(R_{x}^{\star} F(g) R_{y}^{\star} \varphi(g)\right)\right)\right\|_{j} \\
& \leq\left.\sum_{(X)}\left|X_{(1)}\right|_{k}\left|X_{(2)}\right|\right|_{k}\left|\operatorname{Ad}_{x^{-1}}\right|_{k}\left|\operatorname{Ad}_{y^{-1}}\right|_{k}\left|Y_{1}\right|_{k_{1}}\left|Y_{2}\right|_{k_{2}} \\
& \quad \times \sup _{Z_{1} \in \mathcal{U}_{k+k_{1}}(\mathfrak{g})} \frac{\left\|\widetilde{Z}_{1} F(g x)\right\|_{j}}{\left|Z_{1}\right|_{k+k_{1}}} \sup _{Z_{2} \in \mathcal{U}_{k+k_{2}}(\mathfrak{g})} \frac{\left\|\widetilde{Z}_{2} \varphi(g y)\right\|_{j}}{\left|Z_{2}\right|_{k+k_{2}}} \\
& \leq \sum_{(X)}\left|X_{(1)}\right|_{k}\left|X_{(2)}\right|_{k}\left|\operatorname{Ad}_{x^{-1}}\right|_{k}\left|\operatorname{Ad}_{y^{-1}}\right|_{k}\left|Y_{1}\right|_{k_{1}}\left|Y_{2}\right|_{k_{2}} \\
& \quad \times \mu_{j}(g x) \mu_{\phi, 1}^{-N}(g y)\|F\|_{j, k+k_{1}, \underline{\mu}}\|\varphi\|_{j, k+k_{2}, N}
\end{aligned}
$$

which by Lemma 1.5, Lemma 1.14 and the estimate (1.6) is bounded by a constant times

$$
|X|_{k}\left|Y_{1}\right|_{k_{1}}\left|Y_{2}\right|_{k_{2}} \mathfrak{d}_{G \times G}(x, y)^{2 k} \mu_{j}(g x) \mu_{\phi, 1}^{-N}(g y)\|F\|_{j, k+k_{1}, \underline{\mu}}\|\varphi\|_{j, k+k_{2}, N} .
$$

Setting $(L, R)$ for the sub-multiplicative degree of $\mu_{\phi}$ and using

$$
\mu_{\phi, 1}^{-1}(g y) \leq C^{1 / L} \mu_{\phi, 1}^{-1 / L}(g) \mu_{\phi, 1}^{R / L}\left(y^{-1}\right), \quad y, g \in G,
$$


we see that (1.35) is (up to a constant) bounded by

$$
\begin{aligned}
|X|_{k}\left|Y_{1}\right|_{k_{1}}\left|Y_{2}\right|_{k_{2}} \mathfrak{d}_{G \times G}(x, y)^{2 k} \mu_{j}^{L_{j}}(g) \mu_{j}^{R_{j}}(x) \mu_{\phi, 1}^{-N / L}(g) \mu_{\phi, 1}^{N R / L}\left(y^{-1}\right) \\
\times\|F\|_{j, k+k_{1}, \underline{\mu}}\|\varphi\|_{j, k+k_{2}, N} .
\end{aligned}
$$

Now, given $n \in \mathbb{N}$, chose $N_{n}, M_{n} \in \mathbb{N}$ such that

$$
\mu_{j}^{L_{j}} \mu_{\phi, 1}^{-N_{n} / L} \leq \mu_{\phi, 1}^{-n}, \quad \text { and } \quad \mu_{\phi, 1}^{N_{n} R / L} \leq \mu_{\phi, 1}^{M_{n}} .
$$

Then, set

$$
\nu_{j, k, n}(x, y):=\mu_{j}^{R_{j}}(x) \mu_{\phi, 1}^{\vee M_{n}}(y) \mathfrak{d}_{G \times G}^{2 k}(x, y), \quad j, k, n \in \mathbb{N} .
$$

This entails that (1.35) is bounded by

$$
|X|_{k}\left|Y_{1}\right|_{k_{1}}\left|Y_{2}\right|_{k_{2}} \mu_{\phi, 1}^{-n}(g) \mu_{j, k, n}(x, y)\|F\|_{j, k+k_{1}, \underline{\mu}}\|\varphi\|_{j, k+k_{2}, N_{n}},
$$

which, for a finite constant $C\left(j, k, n, k_{1}, k_{2}\right)>0$, finally gives

$$
\|(\mathcal{R} \otimes \mathcal{R})(F, \varphi)\|_{(j, k, n),\left(k_{1}, k_{2}\right), \underline{\nu}} \leq C\left(j, k, n, k_{1}, k_{2}\right)\|F\|_{j, k+k_{1}, \underline{\mu}}\|\varphi\|_{j, k+k_{2}, N_{n}},
$$

proving the claim.

Observe that $1 \otimes \mu_{\phi, 1}$ is tempered on $G \times G$. By Remark 1.2 and since the inversion map on a tempered group is a tempered map, we deduce that $1 \otimes \mu_{\phi, 1}^{\vee}$ is tempered on $G \times G$ too. Hence, when $\mu \otimes 1$ is also tempered on $G \times G$, so is the family of weights $\underline{\nu}$ given in the Lemma 1.49. We then deduce the following important consequence of the latter:

Proposition 1.50. Let $(G \times G, S)$ be a tame and admissible tempered pair, $\mathcal{A}$ a Fréchet algebra, $\underline{\mu}$ a family of tempered weights on $G$, such that the family of weights $\underline{\mu} \otimes 1$ is tempered on $G \times G$ and $\mathbf{m} \in \mathcal{B}^{\mu}(G \times G)$ for some tempered weight $\mu$ on $G \times \bar{G}$. Then the bilinear map $\star_{S}$, defined in (1.33), is continuous on $\mathcal{S}^{S}(G, \mathcal{A})$ and one has the continuous bilinear map:

$$
\star_{S}: \mathcal{B}^{\mu}(G, \mathcal{A}) \times \mathcal{S}^{S}(G, \mathcal{A}) \rightarrow \mathcal{S}^{S}(G, \mathcal{A}), \quad(F, \varphi) \mapsto L_{\star_{S}}(F): \varphi \mapsto F \star_{S} \varphi .
$$

REMARK 1.51. In the context of the proposition above, observe that the restriction to $\mathcal{S}^{S}(G, \mathcal{A}) \times \mathcal{S}^{S}(G, \mathcal{A})$ of the bilinear product $\star_{S}$ (1.33), we have the (point-wise and semi-norm-wise) absolutely convergent expression:

$$
\varphi_{1} \star_{S} \varphi_{2}=\int_{G \times G} \mathbf{m}\left(x_{1}, x_{2}\right) \mathbf{E}\left(x_{1}, x_{2}\right) R_{x_{1}^{\prime}}^{\star}\left(\varphi_{1}\right) R_{x_{2}^{\prime}}^{\star}\left(\varphi_{2}\right) \mathrm{d}_{G}\left(x_{1}\right) \mathrm{d}_{G}\left(x_{1}\right) .
$$




\section{CHAPTER 2}

\section{Tempered pairs for Kählerian Lie groups}

The aim of this chapter is to endow each negatively curved Kählerian Lie group with the structure of a tempered, tame and admissible pair. Recall that a Lie group $G$ is called a Kählerian Lie group when it is endowed with an invariant Kähler structure, i.e. a left-invariant complex structure $\mathbf{J}$ together with a left-invariant Riemannian metric $\mathbf{g}$ such that the triple $(G, \mathbf{J}, \mathbf{g})$ constitutes a Kähler manifold. Within the present memoir, we will be concerned with Kählerian Lie groups whose sectional curvature is negative. We call them negatively curved.

In section 2.1 we briefly review the theory of normal $\mathbf{j}$-algebras and associated normal $\mathbf{j}$-groups, which in turn gives a classification result for negatively curved Kählerian Lie groups. In section 2.2, we explain how an elementary normal $\mathbf{j}$ group is naturally endowed with a structure of a symplectic symmetric space. Such structure is the core of the construction of chapter 5. It is also in this context that we have a clear geometric construction for the phase and amplitude of the kernel underlying our deformation formula. It is then in sections 2.3 and 2.4 that we prove that the phase mentioned above endows a negatively curved Kählerian Lie group with the structure of an admissible and tempered pair.

\subsection{Pyatetskii-Shapiro's theory}

The following definition, due to Pyatetskii-Shapiro [22], describes the infinitesimal structure of negatively curved Kählerian Lie groups.

Definition 2.1. A normal $\mathbf{j}$-algebra is a triple $(\mathfrak{b}, \alpha, \mathbf{j})$ where

(i) $\mathfrak{b}$ is a solvable Lie algebra which is split over the reals, i.e. $\operatorname{ad}_{X}$ has only real eigenvalues for all $X \in \mathfrak{b}$,

(ii) $\mathbf{j}$ is an endomorphism of $\mathfrak{b}$ such that $\mathbf{j}^{2}=-1$ and

$$
[X, Y]+\mathbf{j}[\mathbf{j} X, Y]+\mathbf{j}[X, \mathbf{j} Y]-[\mathbf{j} X, \mathbf{j} Y]=0, \quad X, Y \in \mathfrak{b},
$$

(iii) $\alpha$ is a linear form on $\mathfrak{b}$ such that

$$
\alpha([\mathbf{j} X, X])>0 \text { if } X \neq 0 \quad \text { and } \quad \alpha([\mathbf{j} X, \mathbf{j} Y])=\alpha([X, Y]), \quad X, Y \in \mathfrak{b} .
$$

We quote the following structure result from [22.

Proposition 2.2. The Lie algebra of a negatively curved Kählerian Lie group always carries a structure of normal $\mathbf{j}$-algebra.

If $\mathfrak{b}^{\prime}$ is a subalgebra of $\mathfrak{b}$ which is invariant by $\mathbf{j}$, then $\left(\mathfrak{b}^{\prime},\left.\alpha\right|_{\mathfrak{b}^{\prime}},\left.\mathbf{j}\right|_{\mathfrak{b}^{\prime}}\right)$ is again a normal $\mathbf{j}$-algebra, called a $\mathbf{j}$-subalgebra of $(\mathfrak{b}, \alpha, \mathbf{j})$. A $\mathbf{j}$-subalgebra whose underlying Lie algebra $\mathfrak{b}^{\prime}$ is an ideal of $\mathfrak{b}$ is called a $\mathbf{j}$-ideal.

EXAMPLE 2.3. Every Iwasawa factor $A N$ of the simple Lie group $S U(1, n)$ is naturally a negatively curved Kählerian Lie group. Indeed, denoting by $K \simeq U(n)$ 
a maximal compact subgroup of $S U(1, n)$, one knows that the associated symmetric space $G / K$ is a negatively curved Kählerian $S U(1, n)$-manifold. The associated Iwasawa decomposition $S U(1, n)=A N K$ then yields a global diffeomorphism between $G / K$ and $A N$. Transporting to $A N$ the Kähler structure of $G / K$ under the latter diffeomorphism, then endows $A N$ with a negatively curved Kählerian Lie group structure, called elementary after Pyatetskii-Shapiro.

The infinitesimal structure underlying an elementary normal $\mathbf{j}$-group (cf. the above Example 2.3) may be precisely described as follows. Let $\left(V, \omega^{0}\right)$ be a symplectic vector space of real dimension $2 d$. We consider the associated Heisenberg Lie algebra $\mathfrak{h}:=V \oplus \mathbb{R} E$. That is, $\mathfrak{h}$ is the central extension of the Abelian Lie algebra $V$, with brackets given by

$$
\left[v_{1}, v_{2}\right]:=\omega^{0}\left(v_{1}, v_{2}\right) E, \quad v_{1}, v_{2} \in V, \quad[E, X]:=0, \quad X \in \mathfrak{h} .
$$

Definition 2.4. Let $\mathfrak{a}$ be a one-dimensional real Lie algebra, with generator $H$. We consider the split extension of Lie algebras:

$$
0 \rightarrow \mathfrak{h} \rightarrow \mathfrak{s}:=\mathfrak{a} \ltimes_{\rho_{\mathfrak{h}}} \mathfrak{h} \rightarrow \mathfrak{a} \rightarrow 0,
$$

with extension homomorphism $\rho_{\mathfrak{h}}: \mathfrak{a} \rightarrow \operatorname{Der}(\mathfrak{h})$ given by

$$
\rho_{\mathfrak{h}}(H)(v+t E):=[H, v+t E]:=v+2 t E, \quad v \in V, \quad t \in \mathbb{R} .
$$

The Lie algebra $\mathfrak{s}$ is called elementary normal. Last, we denote by $\mathbb{S}$ the connected simply connected Lie group whose Lie algebra is $\mathfrak{s}$ and we call the latter an elementary normal j-group.

Note that $\mathbb{S}$ is a solvable group of real dimension $2 d+2$ and if $V=\{0\}, \mathbb{S}$ is isomorphic to the affine group of the real line. It turns out that every negatively curved Kählerian Lie group can be decomposed into elementary pieces: at the infinitesimal level, one has the following result, due to Pyatetskii-Shapiro [22].

Proposition 2.5. Let $(\mathfrak{b}, \alpha, \mathbf{j})$ be a normal $\mathbf{j}$-algebra. Then, there exist $\mathfrak{z}$, a one-dimensional ideal of $\mathfrak{b}$ and $V$, a vector subspace of $\mathfrak{b}$, such that setting $\mathfrak{a}:=\mathbf{j} \mathfrak{z}$, the algebra $\mathfrak{s}:=\mathfrak{a} \oplus V \oplus \mathfrak{z}$ underlies an elementary normal $\mathbf{j}$-ideal of $\mathfrak{b}$. Moreover, the associated extension sequence

$$
0 \longrightarrow \mathfrak{s} \longrightarrow \mathfrak{b} \longrightarrow \mathfrak{b}^{\prime} \longrightarrow 0
$$

is split as a sequence of normal $\mathbf{j}$-algebras and such that:

$$
\left[\mathfrak{b}^{\prime}, \mathfrak{a} \oplus \mathfrak{z}\right]=0 \quad \text { and } \quad\left[\mathfrak{b}^{\prime}, V\right] \subset V .
$$

In particular, every normal $\mathbf{j}$-algebra $\mathfrak{b}$ admits a decomposition as a sequence of split extensions of elementary normal $\mathbf{j}$-algebras $\mathfrak{s}_{i}, i=1, \ldots, N$, of real dimension $2 d_{i}+2, d_{i} \in \mathbb{N}:$

$$
\left(\ldots\left(\mathfrak{s}_{N} \ltimes \mathfrak{s}_{N-1}\right) \ltimes \cdots \ltimes \mathfrak{s}_{2}\right) \ltimes \mathfrak{s}_{1},
$$

such that for all $i=1, \ldots, N-1$

$$
\left[\left(\mathfrak{s}_{N} \ltimes \ldots\right) \ltimes \mathfrak{s}_{i+1}, \mathfrak{a}_{i} \oplus \mathfrak{z}_{i}\right]=0 \quad \text { and } \quad\left[\left(\mathfrak{s}_{N} \ltimes \ldots\right) \ltimes \mathfrak{s}_{i+1}, V_{i}\right] \subset V_{i}
$$

Definition 2.6. A normal $\mathbf{j}$-group $\mathbb{B}$, consists of a connected simply connected Lie group that admits a normal $\mathbf{j}$-algebra as Lie algebra, i.e. $\mathbb{B}=\exp \{\mathfrak{b}\}$, where $\mathfrak{b}$ is a normal $\mathbf{j}$-algebra. 
At the group level, for $i=1, \ldots, N-1$, call $\mathbf{R}^{i}$ the extension homomorphism at each step:

$$
\mathbf{R}^{i} \in \operatorname{Hom}\left(\left(\mathbb{S}_{N} \ltimes \ldots\right) \ltimes \mathbb{S}_{i+1}, \operatorname{Aut}\left(\mathbb{S}_{i}\right)\right) .
$$

The conditions given in (2.2) implies that $\mathbf{R}^{i}$ takes values in $\operatorname{Sp}\left(V_{i}, \omega_{i}^{0}\right)$, where $\left(V_{i}, \omega_{i}^{0}\right)$ denotes the symplectic vector space attached to $\mathbb{S}_{i}$.

\subsection{Geometric structures on elementary normal j-groups}

In this section, we review the properties of a symplectic symmetric space structure every elementary normal $\mathbf{j}$-group is naturally endowed with. The phase function with respect to which an admissible tempered pair will be associated to later on, was defined in $[\mathbf{3}$ in terms of this symplectic symmetric space structure. We start with the definition of a symplectic symmetric space as in 1 which is an adaptation to the symplectic case of the notion of symmetric space as introduced by $\mathrm{O}$. Loos [21].

DEFINITION 2.7. A symplectic symmetric space is a triple $(M, s, \omega)$ where

(i) $M$ is a connected smooth manifold,

(ii) $s$ is a smooth map

$$
s: M \times M \rightarrow M, \quad(x, y) \mapsto s_{x}(y):=s(x, y),
$$

such that:

(ii.1) For every $x \in M$, the partial map $s_{x}: M \rightarrow M$ is an involutive diffeomorphism admitting $x$ as isolated fixed point. The diffeomorphism $s_{x}$ is called the symmetry at point $x$.

(ii.2) For all points $x$ and $y$ in $M$, the following relation holds:

$$
s_{x} \circ s_{y} \circ s_{x}=s_{s_{x}(y)} .
$$

(iii) $\omega$ is a closed 1 and non-degenerate two-form on $M$ that is invariant under the symmetries:

$$
s_{x}^{\star} \omega=\omega, \quad \forall x \in M .
$$

A morphism between two symplectic symmetric spaces is defined as a symplectomorphism that intertwines the symmetries.

Symplectic symmetric spaces always carry a preferred Lie group of transformations [1]:

DEFINITION 2.8. The automorphism group of a symplectic symmetric space $(M, s, \omega)$ is constituted by the symplectomorphisms $\varphi \in \operatorname{Symp}(M, \omega)$ which are covariant under the symmetries:

$$
\varphi \circ s_{x}=s_{\varphi(x)} \circ \varphi, \quad \forall x \in M .
$$

It is a Lie subgroup of $\operatorname{Symp}(M, \omega)$ that acts transitively on $M$ and it is denoted by $\operatorname{Aut}(M, s, \omega)$. Its Lie algebra is called the derivation algebra of $(M, s, \omega)$ and is denoted by $\mathfrak{a u t}(M, s, \omega)$.

\footnotetext{
${ }^{1}$ The closedness condition is, in fact, redundant, see $\mathbf{1}$.
} 
We now pass to the particular case of a given $2 d+2$-dimensional elementary normal j-group $\mathbb{S}$ with associated symplectic form $\omega^{\mathbb{S}}$. Let $a, t \in \mathbb{R}$ and $v \in V \simeq \mathbb{R}^{2 d}$. The following identification will always be understood:

$$
\mathbb{R}^{2 d+2} \rightarrow \mathfrak{s}, \quad x:=(a, v, t) \mapsto a H+v+t E .
$$

The following result is extracted from $3,6,8$ :

Proposition 2.9. Let $\mathbb{S}$ be an elementary normal $\mathbf{j}$-group.

(i) The map

$$
\mathfrak{s} \rightarrow \mathbb{S}, \quad(a, v, t) \mapsto \exp (a H) \exp (v+t E)=\exp (a H) \exp (v) \exp (t E),
$$

is a global Darboux chart on $\left(\mathbb{S}, \omega^{\mathbb{S}}\right)$ in which the symplectic structure reads:

$$
\omega^{\mathbb{S}}:=2 \mathrm{~d} a \wedge \mathrm{d} t+\omega^{0} .
$$

(ii) Setting furthermore

$$
s_{(a, v, t)}\left(a^{\prime}, v^{\prime}, t^{\prime}\right):=
$$

$\left(2 a-a^{\prime}, 2 v \cosh \left(a-a^{\prime}\right)-v^{\prime}, 2 t \cosh \left(2 a-2 a^{\prime}\right)-t^{\prime}+\omega^{0}\left(v, v^{\prime}\right) \sinh \left(a-a^{\prime}\right)\right)$,

defines a symplectic symmetric space structure $\left(\mathbb{S}, s, \omega^{\mathbb{S}}\right)$ on the elementary normal $\mathbf{j}$-group $\mathbb{S}$.

(iii) The left action $L_{x}: \mathbb{S} \rightarrow \mathbb{S}, x^{\prime} \mapsto x . x^{\prime}$, defines a injective Lie group homomorphism

$$
L: \mathbb{S} \rightarrow \operatorname{Aut}\left(\mathbb{S}, s, \omega^{\mathbb{S}}\right) .
$$

In the coordinates (2.4), we have

$$
x \cdot x^{\prime}=(a, v, t) \cdot\left(a^{\prime}, v^{\prime}, t^{\prime}\right)=\left(a+a^{\prime}, e^{-a^{\prime}} v+v^{\prime}, e^{-2 a^{\prime}} t+t^{\prime}+\frac{1}{2} e^{-a^{\prime}} \omega^{0}\left(v, v^{\prime}\right)\right) \text {. }
$$

and

$$
x^{-1}=(a, v, t)^{-1}=\left(-a,-e^{a} v,-e^{2 a} t\right) .
$$

(iv) The action $\mathbf{R}: \operatorname{Sp}\left(V, \omega^{0}\right) \times \mathbb{S} \rightarrow \mathbb{S},(A,(a, v, t)) \mapsto \mathbf{R}_{A}(a, v, t):=(a, A v, t)$ by automorphisms of the normal $\mathbf{j}$-group $\mathbb{S}$ induces an injective Lie group homomorphism:

$$
\mathbf{R}: \operatorname{Sp}\left(V, \omega^{0}\right) \rightarrow \operatorname{Aut}\left(\mathbb{S}, s, \omega^{\mathbb{S}}\right), \quad A \mapsto \mathbf{R}_{A} .
$$

In fact, $\operatorname{Sp}\left(V, \omega^{0}\right) \simeq \operatorname{Aut}(\mathbb{S}) \cap \operatorname{Aut}\left(\mathbb{S}, s, \omega^{\mathbb{S}}\right)$.

Note that in the coordinates (2.4), the modular function of $\mathbb{S}, \Delta_{\mathbb{S}}$, reads $e^{(2 d+2) a}$.

We now pass to the definition of the three-point phase on $\mathbb{S}$. For this we need the notion of "double geodesic triangle" as introduced by A. Weinstein $\mathbf{3 6}$ and Z. Qian 23.

Definition 2.10. Let $(M, s)$ be a symmetric space. A midpoint map on $M$ is a smooth map

$$
M \times M \rightarrow M, \quad(x, y) \mapsto \operatorname{mid}(x, y),
$$

such that, for all points $x, y$ in $M$ :

$$
s_{\operatorname{mid}(x, y)}(x)=y .
$$

REMARK 2.11. When it exists, such a midpoint map on a symmetric space $(M, s)$ is necessarily unique (see Lemma 2.1 .6 of $[34]$ ). 
REMARK 2.12. Observe that in the case where the partial maps $s^{y}: M \rightarrow M$, $x \mapsto s_{x}(y)$ are global diffeomorphisms of $M$, a midpoint map exists and is given by:

$$
\operatorname{mid}(x, y):=\left(s^{x}\right)^{-1}(y) \text {. }
$$

Note that in this case, every $\varphi \in \operatorname{Aut}(M, s)$ intertwines the midpoints. Indeed, since for all $x, y \in M$ we have $\varphi\left(s_{y}(x)\right)=s_{\varphi(y)}(\varphi(x))$, we get

$$
\varphi(\operatorname{mid}(x, y))=\operatorname{mid}(\varphi(x), \varphi(y)) .
$$

An immediate computation shows that a midpoint map always exists on the symplectic symmetric space attached to an elementary normal $\mathbf{j}$-group:

LEMMA 2.13. For the symmetric space $(\mathbb{S}, s)$ underlying an elementary normal $\mathbf{j}$-group, the associated partial maps are global diffeomorphisms. In the coordinates (2.4), we have:

$$
\begin{aligned}
& \left(s^{\left(a_{0}, v_{0}, t_{0}\right)}\right)^{-1}(a, v, t)= \\
& \left(\frac{a+a_{0}}{2}, \frac{v+v_{0}}{2 \cosh \left(\frac{a-a_{0}}{2}\right)}, \frac{t+t_{0}}{2 \cosh \left(a-a_{0}\right)}-\omega^{0}\left(v, v_{0}\right) \frac{\sinh \left(\frac{a-a_{0}}{2}\right)}{4 \cosh \left(a-a_{0}\right) \cosh \left(\frac{a-a_{0}}{2}\right)}\right) .
\end{aligned}
$$

The following statement is proved in $\mathbf{3}$.

Proposition 2.14. Let $\mathbb{S}$ be an elementary normal $\mathbf{j}$-group.

(i) The Kähler manifold $\mathbb{S}$ is strictly geodesically complete: two points determine a unique geodesic arc.

(ii) The "medial triangle" three-point function

$$
\Phi: \mathbb{S}^{3} \rightarrow \mathbb{S}^{3}, \quad\left(x_{1}, x_{2}, x_{3}\right) \mapsto\left(\operatorname{mid}\left(x_{1}, x_{2}\right), \operatorname{mid}\left(x_{2}, x_{3}\right), \operatorname{mid}\left(x_{3}, x_{1}\right)\right),
$$

is a $\mathbb{S}$-equivariant (under the left regular action) global diffeomorphism.

Since our space $\mathbb{S}$ has trivial de Rham cohomology in degree two, any three points $(x, y, z) \in \mathbb{S}^{3}$ define an oriented geodesic triangle $T(x, y, z)$ whose symplectic area is well-defined by integrating the two-form $\omega^{\mathbb{S}}$ on any surface admitting $T(x, y, z)$ as boundary. With a slight abuse of notation, we set

$$
\operatorname{Area}(x, y, z):=\int_{T(x, y, z)} \omega^{\mathbb{S}} .
$$

DEFINITION 2.15. The canonical two-point phase associated to an elementary normal $\mathbf{j}$-group is defined by

$$
S_{\text {can }}^{\mathbb{S}}\left(x_{1}, x_{2}\right):=\operatorname{Area}\left(\Phi^{-1}\left(e, x_{1}, x_{2}\right)\right) \in C^{\infty}\left(\mathbb{S}^{2}, \mathbb{R}\right),
$$

where $e:=(0,0,0)$ denotes the unit element in $\mathbb{S}$. In the coordinates (2.4), one has the explicit expression:

$$
S_{\mathrm{can}}^{\mathbb{S}}\left(x_{1}, x_{2}\right)=t_{2} \sinh 2 a_{1}-t_{1} \sinh 2 a_{2}+\omega^{0}\left(v_{1}, v_{2}\right) \cosh a_{1} \cosh a_{2} .
$$

The canonical two-point amplitude associated to an elementary normal $\mathbf{j}$-group is defined by

$$
A_{\text {can }}^{\mathbb{S}}\left(x_{1}, x_{2}\right):=\operatorname{Jac}_{\Phi^{-1}}\left(e, x_{1}, x_{2}\right)^{1 / 2} \in C^{\infty}\left(\mathbb{S}^{2}, \mathbb{R}\right) .
$$

In the coordinates (2.4), it reads

$$
\begin{aligned}
& A_{\text {can }}^{\mathbb{S}}\left(x_{1}, x_{2}\right)= \\
& \left(\cosh a_{1} \cosh a_{2} \cosh \left(a_{1}-a_{2}\right)\right)^{d}\left(\cosh 2 a_{1} \cosh 2 a_{2} \cosh \left(2 a_{1}-2 a_{2}\right)\right)^{1 / 2} .
\end{aligned}
$$




\subsection{Tempered pair for elementary normal j-groups}

The aim of this technical section is to prove that the pair $\left(\mathbb{S} \times \mathbb{S}, S_{\text {can }}^{\mathbb{S}}\right)$ is tempered, admissible and tame. We start by splitting the $2 d$-dimensional symplectic vector space $\left(V, \omega^{0}\right)$ associated to an elementary normal $\mathbf{j}$-group $\mathbb{S}$ into a direct sum of two Lagrangian subspaces in symplectic duality:

$$
V=\mathfrak{l}^{\star} \oplus \mathfrak{l} \text {. }
$$

The following result establishes temperedness.

Lemma 2.16. The pair $\left(\mathbb{S} \times \mathbb{S}, S_{\text {can }}^{\mathbb{S}}\right)$ is tempered. Moreover, the Jacobian of the map

$$
\phi: \mathbb{S} \times \mathbb{S} \rightarrow(\mathfrak{s} \oplus \mathfrak{s})^{\star}, \quad g \mapsto\left[X \in \mathfrak{s} \oplus \mathfrak{s} \mapsto\left(\widetilde{X} . S_{\text {can }}^{\mathbb{S}}\right)(g)\right],
$$

is proportional to $\left(A_{\text {can }}^{\mathbb{S}}\right)^{2}$.

Proof. Let us fix $\left\{f_{j}\right\}_{j=1}^{d}$, a basis of $\mathfrak{l}^{\star}$ to which we associate $\left\{e_{j}\right\}_{j=1}^{d}$ the symplectic-dual basis of $\mathfrak{l}$, i.e. it is defined by $\omega^{0}\left(f_{i}, e_{j}\right)=\delta_{i, j}$. We let $E$ the central element of the Heisenberg Lie algebra $\mathfrak{h} \subset \mathfrak{s}$ and $H$ the generator of $\mathfrak{a}$ in the one dimensional split extension which defines the Lie algebra $\mathfrak{s}$ :

$$
0 \rightarrow \mathfrak{h} \rightarrow \mathfrak{s} \rightarrow \mathfrak{a} \rightarrow 0 .
$$

Accordingly, we consider the following basis of $\mathfrak{s} \oplus \mathfrak{s}$ :

$$
\begin{aligned}
H_{1}:=H \oplus\{0\}, \quad H_{2} & :=\{0\} \oplus H, \\
f_{j}^{1}:=f_{j} \oplus\{0\}, \quad f_{j}^{2}:= & \{0\} \oplus f_{j}, \\
e_{j}^{1}:=e_{j} \oplus\{0\}, \quad e_{j}^{2} & :=\{0\} \oplus e_{j}, \\
E_{1}:=E \oplus\{0\}, \quad E_{2} & :=\{0\} \oplus E,
\end{aligned}
$$

where the index $j$ runs from 1 to $d=\operatorname{dim}(V) / 2$. From Proposition 2.9 iii) and with the notation $v=(x, y) \in \mathfrak{l}^{\star} \oplus \mathfrak{l}=V$, we see that the left-invariant vector fields on $\mathbb{S}$ are given by:

$$
\begin{array}{ccc}
\widetilde{H} & = & \partial_{a}-\sum_{j=1}^{d}\left(x_{j} \partial_{x_{j}}+y_{j} \partial_{y_{j}}\right)-2 t \partial_{t} \\
\widetilde{f}_{j} & = & \partial_{x_{j}}-\frac{y_{j}}{2} \partial_{t} \\
\widetilde{\widetilde{e}}_{j} & = & \partial_{y_{j}}+\frac{x_{j}}{2} \partial_{t} \\
\widetilde{E} & = & \partial_{t}
\end{array}
$$

Thus, we find

$$
\begin{aligned}
& \widetilde{H}_{1} S_{\text {can }}^{\mathbb{S}}=2 t_{2} \cosh 2 a_{1}+2 t_{1} \sinh 2 a_{2}-\omega^{0}\left(v_{1}, v_{2}\right) e^{-a_{1}} \cosh a_{2}, \\
& \widetilde{H}_{2} S_{\text {can }}^{\mathbb{S}}=-2 t_{1} \cosh 2 a_{2}-2 t_{2} \sinh 2 a_{1}-\omega^{0}\left(v_{1}, v_{2}\right) e^{-a_{2}} \cosh a_{1}, \\
& \widetilde{E}_{1} S_{\text {can }}^{\mathbb{S}}=-\sinh 2 a_{2}, \\
& \widetilde{E}_{2} S_{\text {can }}^{\mathbb{S}}=\sinh 2 a_{1}, \\
& \widetilde{f}_{j}^{1} S_{\text {can }}^{\mathbb{S}}=y_{2}^{j} \cosh a_{1} \cosh a_{2}+\frac{1}{2} y_{1}^{j} \sinh 2 a_{2}, \\
& \widetilde{f}_{j}^{2} S_{\text {can }}^{\mathbb{S}}=-y_{1}^{j} \cosh a_{1} \cosh a_{2}-\frac{1}{2} y_{2}^{j} \sinh 2 a_{1}, \\
& \widetilde{e}_{j}^{1} S_{\text {can }}^{\mathbb{S}}=-x_{2}^{j} \cosh a_{1} \cosh a_{2}-\frac{1}{2} x_{1}^{j} \sinh 2 a_{2}, \\
& \widetilde{e}_{j}^{2} S_{\text {can }}^{\mathbb{S}}=x_{1}^{j} \cosh a_{1} \cosh a_{2}+\frac{1}{2} x_{2}^{j} \sinh 2 a_{1} .
\end{aligned}
$$


A computation then shows that the Jacobian of the map $\phi: \mathbb{S} \times \mathbb{S} \rightarrow(\mathfrak{s} \oplus \mathfrak{s})^{\star}$, underlying Definition 1.22, is given by

$$
\begin{gathered}
2^{2 d+2}\left(\cosh a_{1} \cosh a_{2} \cosh \left(a_{1}-a_{2}\right)\right)^{2 d} \cosh 2 a_{1} \cosh 2 a_{2} \cosh 2\left(a_{1}-a_{2}\right) \\
=2^{2 d+2} A_{\text {can }}^{\mathbb{S}}\left(x_{1}, x_{2}\right)^{2} \geq 2^{2 d+2},
\end{gathered}
$$

and hence $\phi$ is a global diffeomorphism. It is also clear from Proposition 2.9 iii), that the multiplication and inversion maps on $\mathbb{S} \times \mathbb{S}$ are tempered functions in the coordinates (2.9). Therefore, the pair $\left(\mathbb{S} \times \mathbb{S}, S_{\text {can }}^{\mathbb{S}}\right)$ is tempered.

REMARK 2.17. Note that the formal adjoints of the left invariant vector fields (2.8), with respect to the inner product of $L^{2}(\mathbb{S})$ read:

$$
\widetilde{H}^{*}=-\widetilde{H}+2 d+2, \quad \widetilde{f}_{j}^{*}=-\widetilde{f}_{j}, \quad \widetilde{e}_{j}^{*}=-\widetilde{e}_{j}, \quad \widetilde{E}^{*}=-\widetilde{E},
$$

so that the assumption (1.16) is trivially satisfied.

We will now prove that the tempered pair $\left(\mathbb{S} \times \mathbb{S}, S_{\text {can }}^{\mathbb{S}}\right)$ is admissible and tame. For this, we need a decomposition of the Lie algebra $\mathfrak{s}$ and we shall use the following one:

$$
\mathfrak{s}=\bigoplus_{k=0}^{3} V_{k} \quad \text { where } \quad V_{0}:=\mathfrak{a}, \quad V_{1}:=\mathfrak{l}^{\star}, \quad V_{2}:=\mathfrak{l} \quad \text { and } \quad V_{3}:=\mathbb{R} E
$$

Note that both $V_{0}$ and $V_{3}$ are of dimension one, while $V_{1}$ and $V_{2}$ are $d$-dimensional. Accordingly, we consider the decompositions of $\mathfrak{s} \oplus \mathfrak{s}$ given by

$$
\mathfrak{s} \oplus\{0\}=\bigoplus_{k=0}^{3} V_{1, k} \quad \text { and } \quad\{0\} \oplus \mathfrak{s}=\bigoplus_{k=0}^{3} V_{2, k},
$$

where the subspaces $V_{i, k}, i=1,2$, of each factor correspond respectively to the subspaces $V_{k}$ of $\mathfrak{s}$ within the decomposition (2.10). We then set:

$$
\mathfrak{V}_{k}:=V_{1, k} \oplus V_{2, k} \quad \text { and } \quad \mathfrak{s} \oplus \mathfrak{s}=\bigoplus_{k=0}^{3} \mathfrak{V}_{k}
$$

by which we mean that there are four subspaces involved in the ordered decomposition of $\mathfrak{s} \oplus \mathfrak{s}$. We also let

$$
\mathfrak{V}^{(k)}:=\bigoplus_{n=0}^{k} \mathfrak{V}_{k}, \quad k=0,1,2,3,
$$

as in (1.15) and we let $\mathcal{U}\left(\mathfrak{V}^{(k)}\right)$ be the unital subalgebra of $\mathcal{U}(\mathfrak{s} \oplus \mathfrak{s})$ generated by $\mathfrak{V}^{(k)}$ as in (0.8). Accordingly, we consider the associated tempered coordinates (1.12):

$$
x_{i, 0}:=\widetilde{H}_{i} S_{\text {can }}^{\mathbb{S}}, \quad x_{i, 1}^{j}:=\tilde{f}_{j}^{i} S_{\text {can }}^{\mathbb{S}}, \quad x_{i, 2}^{j}:=\tilde{e}_{j}^{i} S_{\text {can }}^{\mathbb{S}}, \quad x_{i, 3}:=\widetilde{E}_{i} S_{\text {can }}^{\mathbb{S}},
$$

with $i=1,2, j=1, \ldots, d$ and we use the vector notations:

$$
\begin{aligned}
& \vec{x}_{0}:=\left(x_{1,0}, x_{2,0}\right) \in \mathbb{R}^{2}, \\
& \vec{x}_{1}:=\left(x_{1,1}, x_{2,1}\right):=\left(\left(x_{1,1}^{j}\right)_{j=1}^{d},\left(x_{2,1}^{j}\right)_{j=1}^{d}\right) \in \mathbb{R}^{2 d}, \\
& \vec{x}_{2}:=\left(x_{1,2}, x_{2,2}\right):=\left(\left(x_{1,2}^{j}\right)_{j=1}^{d},\left(x_{2,2}^{j}\right)_{j=1}^{d}\right) \in \mathbb{R}^{2 d}, \\
& \vec{x}_{3}:=\left(x_{1,3}, x_{2,3}\right) \in \mathbb{R}^{2} .
\end{aligned}
$$


According to the notations $(a, v, t) \in \mathbb{R} \times \mathbb{R}^{2 d} \times \mathbb{R} \simeq \mathbb{S}$ and $v=(x, y) \in \mathfrak{l}^{\star} \oplus \mathfrak{l}=V$, we set

$\vec{a}:=\left(a_{1}, a_{2}\right) \in \mathbb{R}^{2}, \quad \vec{x}=\left(x_{1}, x_{2}\right) \in \mathbb{R}^{2 d}, \quad \vec{y}=\left(y_{1}, y_{2}\right) \in \mathbb{R}^{2 d}, \quad \vec{t}:=\left(t_{1}, t_{2}\right) \in \mathbb{R}^{2}$.

We consider the functions

$$
s_{12}:=t_{2} \sinh 2 a_{1}-t_{1} \sinh 2 a_{2}, \quad \Omega_{12}:=\omega^{0}\left(v_{1}, v_{2}\right), \quad \gamma_{12}:=\cosh a_{1} \cosh a_{2},
$$

in term of which we have

$$
S_{\text {can }}^{\mathbb{S}}=s_{12}+\gamma_{12} \Omega_{12} .
$$

Introducing last

$$
\begin{aligned}
& A:=\left(\begin{array}{cc}
\sinh 2 a_{2} & \cosh 2 a_{1} \\
-\cosh 2 a_{2} & -\sinh 2 a_{1}
\end{array}\right), \\
& B:=\left(\begin{array}{cc}
-\frac{1}{2} \sinh 2 a_{2} & -\cosh a_{1} \cosh a_{2} \\
\cosh a_{1} \cosh a_{2} & \frac{1}{2} \sinh 2 a_{1}
\end{array}\right), \\
& \vec{\gamma}:=\left(\begin{array}{lll}
e^{-a_{1}} \cosh a_{2}, \quad e^{-a_{2}} \cosh a_{1}
\end{array}\right), \quad \vec{\delta}:=\left(\begin{array}{ll}
-\sinh 2 a_{2}, & \sinh 2 a_{1}
\end{array}\right),
\end{aligned}
$$

the relations given in (2.9) can be summarized as:

$$
\vec{x}_{3}=\vec{\delta}, \quad \vec{x}_{2}=B \cdot \vec{x}, \quad \vec{x}_{1}=-B \cdot \vec{y}, \quad \vec{x}_{0}=2 A \cdot \vec{t}-\Omega_{12} \vec{\gamma} .
$$

We first treat the easiest variable $\vec{x}_{3}$, which lead to multipliers $\alpha_{3}$ that satisfy property (ii) of Definition 1.24 with constant $\mu_{3}$ :

Lemma 2.18. Consider an element $X \in \mathcal{U}\left(\mathfrak{V}_{3}\right)$ such that the associated multiplier $\alpha_{X}$ is invertible. Then, for every $Y \in \mathcal{U}\left(\mathfrak{V}^{(3)}\right)=\mathcal{U}(\mathfrak{s} \oplus \mathfrak{s})$ there exists a positive constant $C_{Y}$ such that

$$
\left|\widetilde{Y} \alpha_{X}\right| \leq C_{Y}\left|\alpha_{X}\right|
$$

Proof. Note first that $\mathfrak{V}_{3}$ turns out to be a two-dimensional Abelian Lie algebra. Note also that $\alpha_{E_{i}}, i=1,2$ is independent of the variable $\vec{t}$. Thus, given a two-variables polynomial $P$, we have for $X=P\left(E_{1}, E_{2}\right) \in \mathcal{U}\left(\mathfrak{V}_{3}\right)$ :

$$
\alpha_{X}=P\left(-\sinh 2 a_{2}, \sinh 2 a_{1}\right) .
$$

It also follows from the explicit expression of the left-invariant vector fields given in (2.8) that $\widetilde{Y} \alpha_{X}=0$ for all $Y \in \mathcal{U}\left(\oplus_{k=1}^{3} \mathfrak{V}_{k}\right)$. Hence, it suffices to treat the case of $Y \in \mathcal{U}\left(\mathfrak{V}_{0}\right)$. Observe that the restriction of $\widetilde{H}_{j}$ to functions which depend only on $a_{j}$, equals $\partial_{a_{j}}$. Thus in this case, we see that $\widetilde{Y} \alpha_{X}$ is a polynomial of the same degree as $P$, but in the variables $e^{ \pm a_{1}}$ and $e^{ \pm a_{2}}$. This is enough to conclude when $\alpha_{X}$ is invertible.

Next, we treat the variables $\vec{x}_{2}$ and $\vec{x}_{1}$. We first observe

LEMMA 2.19. There exist finitely many matrices $B_{(r)} \in M_{2}\left(\mathbb{R}\left[e^{ \pm a_{1}}, e^{ \pm a_{2}}\right]\right)$ such that for all integers $N_{1}$ and $N_{2}$, the elements $\widetilde{H}_{1}^{N_{1}} \widetilde{H}_{2}^{N_{2}} B$ consist of a linear combination of the $B_{(r)}$ 's, where the matrix $B$ has been defined in (2.13). The same property holds for the matrix A. 
Proof. Set

$$
D:=\left(\begin{array}{cc}
-\frac{1}{2} \sinh 2 a_{2} & 0 \\
0 & \frac{1}{2} \sinh 2 a_{1}
\end{array}\right), \quad \Gamma:=\gamma_{12}\left(\begin{array}{cc}
0 & -1 \\
1 & 0
\end{array}\right),
$$

and observes that

$$
B=D+\Gamma \quad \text { and } \quad \partial_{a_{i}}^{2} \Gamma=\Gamma, \quad i=1,2 .
$$

The derivatives of $B$ therefore all belong to the space generated by the entries of $D$ and $\Gamma$ and by finitely many of their derivatives. This is enough to conclude since restricted to functions that depend only on the variable $a$, we have $\widetilde{H}=\partial_{a}$. The proof for the matrix $A$ is entirely similar.

We can now deduce what we need for the variables $\vec{x}_{2}$ and $\vec{x}_{1}$.

LEMma 2.20. There exist finitely many tempered functions $\mathbf{m}_{2,(r)}$ (respectively $\left.\mathbf{m}_{1,(r)}\right)$ depending on the variable $\vec{x}_{3}$ only, such that for every element $X \in \mathcal{U}\left(\mathfrak{V}^{(2)}\right)$ (respectively $X \in \mathcal{U}\left(\mathfrak{V}^{(1)}\right)$ ), the element $\widetilde{X} \vec{x}_{2}$ (respectively $\widetilde{X} \vec{x}_{1}$ ) belongs to the space spanned by $\left\{\mathbf{m}_{2,(r)}, \mathbf{m}_{2,(r)} \vec{x}_{2}\right\}$ (respectively $\left\{\mathbf{m}_{1,(r)}, \mathbf{m}_{1,(r)} \vec{x}_{1}\right\}$ ).

Proof. This follows from Lemma 2.19 and the expressions (2.8) for the invariant vector fields. Indeed, the latter implies that for every $X \in \mathcal{U}\left(\bigoplus_{k=1}^{2} \mathfrak{V}_{k}\right)$ (respectively $X \in \mathcal{U}\left(\mathfrak{V}_{1}\right)$ ) of strictly positive homogeneous degree, $\widetilde{X} \vec{x}_{2}$ (respectively $\widetilde{X} \vec{x}_{1}$ ) is either zero or one of the entries of the matrix $B$.

REMARK 2.21. Note that in view of the expressions (2.8) and (2.9) and by symmetry on $\vec{x}_{1}$ and $\vec{x}_{2}$ the assertion in Lemma 2.20 holds for every element $X$ in $\mathcal{U}(\mathfrak{s} \oplus \mathfrak{s})$ for both variables $\vec{x}_{1}$ and $\vec{x}_{2}$.

Last, we go to the variable $\vec{x}_{0}$. The next Lemma is proved using the same type of arguments as in the proof of Lemma 2.19.

Lemma 2.22. There exist finitely many vectors $\gamma_{(r)} \in \mathbb{R}^{2}\left[e^{ \pm a_{1}}, e^{ \pm a_{2}}\right]$ such that for all integers $N_{1}$ and $N_{2}$, the elements $\widetilde{H}_{1}^{N_{1}} \widetilde{H}_{2}^{N_{2}} \gamma$ consist of a linear combination of the $\gamma_{(r)}$ 's.

Observing that $\widetilde{H}_{i} \vec{t}$ is proportional to $t_{i}$ and that $\widetilde{H}_{i} \Omega_{12}=-\Omega_{12}$, the Lemmas 2.19 and 2.22 then yield the following result.

Lemma 2.23. There exist finitely many matrices $M_{(r)} \in M_{2}\left(\mathbb{R}\left[e^{ \pm a_{1}}, e^{ \pm a_{2}}\right]\right)$ and finitely many vectors $v_{(s)} \in \mathbb{R}^{2}\left[e^{a_{1}}, a^{a_{2}}\right]$ such that for all integers $N_{1}$ and $N_{2}$, one has

with

$$
\widetilde{H}_{1}^{N_{1}} \widetilde{H}_{2}^{N_{2}} \vec{x}_{0}=M_{N_{1}, N_{2}} \vec{x}_{0}+\Omega_{12} v_{N_{1}, N_{2}},
$$

$$
M_{N_{1}, N_{2}} \in \operatorname{span}\left\{M_{(r)}\right\} \quad \text { and } \quad v_{N_{1}, N_{2}} \in \operatorname{span}\left\{v_{(s)}\right\} .
$$

The following result is then a consequence of Lemmas 2.18, 2.20 and 2.22,

Corollary 2.24. For every $k=0, \ldots, 3$, there exists a tempered function $0<\mathbf{m}_{k}$ with $\partial_{\vec{x}_{j}} \mathbf{m}_{k}=0$ for every $j \leq k$ and such that for every $X \in \mathcal{U}\left(\mathfrak{V}^{(k)}\right)$, there exists $C_{X}>0$ with

$$
\left|\widetilde{X} \vec{x}_{k}\right| \leq C_{X} \mathbf{m}_{k}\left(1+\left|\vec{x}_{k}\right|\right) .
$$

REMARK 2.25. In fact the function $\mathbf{m}_{0}$ above depends on $\vec{x}_{1}, \vec{x}_{2}, \vec{x}_{3}$ and the functions $\mathbf{m}_{1}$ and $\mathbf{m}_{2}$ depend on $\vec{x}_{3}$ only (and $\mathbf{m}_{3}$ is constant as it should be). 
We are now able to check the admissibility conditions of Definition 1.24, for the tempered pair $\left(\mathbb{S} \times \mathbb{S}, S_{\text {can }}^{\mathbb{S}}\right)$.

Proposition 2.26. Define

$$
\begin{aligned}
X_{0} & :=1-H_{1}^{2}-H_{2}^{2}, \quad X_{1}:=1-\sum_{j=1}^{d}\left(\left(f_{j}^{1}\right)^{2}+\left(f_{j}^{2}\right)^{2}\right), \\
X_{2} & :=1-\sum_{j=1}^{d}\left(\left(e_{j}^{1}\right)^{2}+\left(e_{j}^{2}\right)^{2}\right), \quad X_{3}:=1-E_{1}^{2}-E_{2}^{2} .
\end{aligned}
$$

Then the corresponding multipliers $\alpha_{k}:=e^{-i S_{\text {can }}^{S}} \widetilde{X}_{k} e^{i S_{\text {can }}^{\mathbb{S}}}, k=0, \ldots, 3$, satisfy conditions (i) and (ii) of Definition 1.24.

Proof. We start by observing the following expression of the multiplier:

$$
\alpha_{k}=1+\left|\vec{x}_{k}\right|^{2}-i \beta_{k}, \quad k=0, \ldots, 3,
$$

where

$$
\beta_{k}:=\widetilde{X}_{1, k} x_{1, k}+\widetilde{X}_{2, k} x_{2, k},
$$

with obvious notations. Then we get

$$
\frac{1}{\left|\alpha_{k}\right|^{2}}=\frac{1}{\left(1+\left|\vec{x}_{k}\right|^{2}\right)^{2}+\beta_{k}^{2}} \leq \frac{1}{\left(1+\left|\vec{x}_{k}\right|^{2}\right)^{2}},
$$

and the first condition of Definition 1.24 is satisfied for $C_{k}=1$ and $\rho_{k}=2$. Let now $X \in \mathcal{U}\left(\mathfrak{V}^{(k)}\right)$ be of strictly positive order. Then, using Sweedler's (0.2), notations we get

$$
\widetilde{X} \alpha_{k}=\sum_{(X)}\left(\widetilde{X}_{(1)} \vec{x}_{k}\right) \cdot\left(\widetilde{X}_{(2)} \vec{x}_{k}\right)-i \widetilde{X} \widetilde{X}_{1, k} x_{1, k}-i \widetilde{X} \widetilde{X}_{2, k} x_{2, k} .
$$

Since $X_{(1)}, X_{(2)}, X_{1, k}, X_{2, k} \in \mathcal{U}\left(\mathfrak{V}^{(k)}\right)$, Corollary 2.24 yields

$$
\left|\widetilde{X} \alpha_{k}\right| \leq C_{1} \mathbf{m}_{k}^{2}\left(1+\left|\vec{x}_{k}\right|\right)^{2}+C_{2} \mathbf{m}_{k}\left(1+\left|\vec{x}_{k}\right|\right) .
$$

As $1+\left|\vec{x}_{k}\right|^{2} \leq\left|\alpha_{k}\right|$, the second condition of Definition 1.24 is satisfied for $\mu_{k}=$ $\mathbf{m}_{k}\left(1+\mathbf{m}_{k}\right)$.

Last, we prove tameness for the pair $\left(\mathbb{S} \times \mathbb{S}, S_{\text {can }}^{\mathbb{S}}\right)$. We start by describing the behavior of the modular weight of the group $\mathbb{S}$ :

LEMMA 2.27. Within the chart (2.4), we have the following behavior of the the modular weight $\mathfrak{d}_{\mathbb{S}}$ of an elementary normal $\mathbf{j}$-group $\mathbb{S}$ :

$$
\mathfrak{d}_{\mathbb{S}} \asymp\left[(a, v, t) \mapsto \cosh a+\cosh 2 a+|v|\left(1+e^{2 a}+\cosh a\right)+|t|\left(1+e^{2 a}\right)\right] .
$$

Proof. Within the decomposition $\mathbb{R} H \oplus V \oplus \mathbb{R} E$ of $\mathfrak{s}$, and within the chart (2.4) of $\mathbb{S}$, a quick computation gives

$$
\begin{aligned}
\operatorname{Ad}_{(a, v, t)} & =\left(\begin{array}{ccc}
\mathrm{id} & 0 & 0 \\
-e^{a} v & e^{a} & 0 \\
2 e^{2 a} t & \frac{1}{2} e^{2 a} \omega^{0}(v, .) & e^{2 a}
\end{array}\right), \\
\operatorname{Ad}_{(a, v, t)^{-1}} & =\left(\begin{array}{ccc}
\mathrm{id} & 0 & 0 \\
v & e^{-a} & 0 \\
-2 t & -\frac{1}{2} e^{-a} \omega^{0}(v, .) & e^{-2 a}
\end{array}\right) .
\end{aligned}
$$


Now, the result follows from the equivalence of the operator and Hilbert-Schmidt norms on the finite dimensional vector space $\operatorname{End}(\mathfrak{s})$, together with obvious estimates.

Corollary 2.28. The tempered pair $\left(\mathbb{S} \times \mathbb{S}, S_{\text {can }}^{\mathbb{S}}\right)$ is tame.

Proof. Combining the last statement of Lemma 1.5 with Lemma 2.27, we get

$$
\mathfrak{d}_{\mathbb{S} \times \mathbb{S}}\left(a_{1}, v_{1}, t_{1} ; a_{2}, v_{2}, t_{2}\right) \geq C\left(\cosh a_{1}+\cosh a_{2}+\left|v_{1}\right|+\left|v_{2}\right|+\left|t_{1}\right|+\left|t_{2}\right|\right),
$$

so that with the relations (2.14) in mind, we see that there exists $C^{\prime}>0$ with

$$
\left(\left|\vec{x}_{0}\right|^{2}+\left|\vec{x}_{1}\right|^{2}+\left|\vec{x}_{2}\right|^{2}+\left|\vec{x}_{3}\right|^{2}\right)^{1 / 2} \leq C^{\prime} \mathfrak{d}_{\mathbb{S} \times \mathbb{S}}^{4} .
$$

According to Definition 1.34, we may set $\mu_{\phi}=\mathfrak{d}_{\mathbb{S} \times \mathbb{S}}^{4}$ which is tempered since $\mathfrak{d}_{\mathbb{S} \times \mathbb{S}}$ is by Lemma 1.5 and Lemma 1.21

We summarize all this by stating the main result of this section:

THEOREM 2.29. Let $\mathbb{S}$ be an elementary normal $\mathbf{j}$-group and let $S_{\mathrm{can}}^{\mathbb{S}}$ be the smooth function on $\mathbb{S} \times \mathbb{S}$ given in Definition 2.15. Then, the pair $\left(\mathbb{S} \times \mathbb{S}, S_{\text {can }}^{\mathbb{S}}\right)$ is tempered, admissible and tame.

REMARK 2.30. From Remark 2.21 and the above discussion, we observe that setting $\mathfrak{V}_{12}:=\mathfrak{V}_{1} \oplus \mathfrak{V}_{2}$ yields a decomposition into three subspaces: $\mathfrak{s} \oplus \mathfrak{s}=$ $\mathfrak{V}_{0} \oplus \mathfrak{V}_{12} \oplus \mathfrak{V}_{3}$ also underlying admissibility but with associated elements $X_{0}, X_{3}$ and $X_{12}:=X_{1}+X_{2}$. The corresponding multipliers are $\alpha_{0}, \alpha_{3}$ and $\alpha_{12}=\alpha_{1}+\alpha_{2}$.

\subsection{Tempered pairs for normal j-groups}

Let $\mathbb{B}$ be a normal $\mathbf{j}$-group with Lie algebra $\mathfrak{b}$. We first observe:

Lemma 2.31. Let $\mathbb{B}=\mathbb{B}^{\prime} \ltimes \mathbb{S}_{1}$ be a Pyatetskii-Shapiro decomposition with $\mathbb{S}_{1}$ elementary normal. Then, there exists $C>0$ such that for every $g_{1}=(a, v, t) \in \mathbb{S}_{1}$, $g^{\prime} \in \mathbb{B}^{\prime}$, we have:

$$
\mathfrak{d}_{\mathbb{B}}\left(g_{1} g^{\prime}\right) \geq C\left(\mathfrak{d}_{\mathbb{B}^{\prime}}\left(g^{\prime}\right)+\cosh 2 a+|v|+|t|\right) .
$$

Proof. By Lemma 1.5 we know that there exists $C_{1}>0$ such that for all $g_{1} \in \mathbb{S}_{1}$ and $g^{\prime} \in \mathbb{B}^{\prime}$, we have,

$$
\mathfrak{d}_{\mathbb{B}}\left(g_{1} g^{\prime}\right) \geq C_{1}\left(\mathfrak{d}_{\mathbb{B}^{\prime}}\left(g^{\prime}\right)+\left(1+\left|\operatorname{Ad}_{g_{1}} \mathbf{R}_{g^{\prime}}\right|^{2}+\left|\mathbf{R}_{g^{\prime-1}} \operatorname{Ad}_{g_{1}^{-1}}\right|^{2}\right)^{1 / 2}\right),
$$

In the decomposition $\mathbb{R} H \oplus V \oplus \mathbb{R} E$ of $\mathfrak{s}$, the $\mathbb{B}^{\prime}$-action may be expressed under the following matrix form:

$$
\mathbf{R}_{g^{\prime}}=\left(\begin{array}{ccc}
i \mathrm{~d} & 0 & 0 \\
0 & A\left(g^{\prime}\right) & 0 \\
0 & 0 & i \mathrm{~d}
\end{array}\right)
$$

where $A\left(g^{\prime}\right)$ is the matrix in the linear symplectic group $\operatorname{Sp}\left(V_{1}, \omega_{1}^{0}\right)$ as defined in Proposition 2.9 (iv). From (2.15) and setting as usual $g_{1}=(a, v, t)$, we therefore obtain:

$$
\begin{aligned}
\operatorname{Ad}_{g_{1}} \mathbf{R}_{g^{\prime}} & =\left(\begin{array}{ccc}
\mathrm{id} & 0 & 0 \\
-e^{a} v & e^{a} A\left(g^{\prime}\right) & 0 \\
2 e^{2 a} t & \frac{1}{2} e^{2 a} \omega^{0}\left(v, A\left(g^{\prime}\right) .\right) & e^{2 a}
\end{array}\right), \\
\mathbf{R}_{g^{\prime-1}} \operatorname{Ad}_{g_{1}^{-1}} & =\left(\begin{array}{ccc}
\mathrm{id} & 0 & 0 \\
A\left(g^{\prime}\right)^{-1} v & e^{-a} A\left(g^{\prime}\right)^{-1} & 0 \\
-2 t & -\frac{1}{2} e^{-a} \omega^{0}(v, .) & e^{-2 a}
\end{array}\right) .
\end{aligned}
$$


Using once again the equivalence between operator and Hilbert-Schmidt norms on $\operatorname{End}(\mathfrak{s})$, we deduce for some $C_{2}>0$ :

$$
\begin{aligned}
\left(1+\left|\operatorname{Ad}_{g_{1}} \mathbf{R}_{g^{\prime}}\right|^{2}+\left|\mathbf{R}_{g^{\prime-1}} \operatorname{Ad}_{g_{1}^{-1}}\right|^{2}\right)^{1 / 2} & \geq C_{2}(\cosh 2 a+|v| \cosh a+|t|) \\
& \geq C_{2}(\cosh 2 a+|v|+|t|),
\end{aligned}
$$

and the proof follows.

Now, we let also $\mathfrak{b}=\mathfrak{a} \oplus \mathfrak{n}$ be a decomposition of the Lie algebra of a normal j-group $\mathbb{B}$, with $\mathfrak{n}$ the nilradical of $\mathfrak{b}$ and $\mathfrak{a}$ its orthogonal complement. It follows then that $\mathfrak{a}$ is an abelian subalgebra, so that $\mathfrak{b}=\mathfrak{a} \ltimes \mathfrak{n}$ and the group $\mathbb{B}$ may be identified to its Lie algebra $\mathfrak{b}$ with product

$$
(a, n) \cdot\left(a^{\prime}, n^{\prime}\right)=\left(a+a^{\prime},\left(e^{-\mathrm{ad} a^{\prime}} n\right) \cdot \mathrm{CBH} n^{\prime}\right),
$$

where $n \cdot \mathrm{CBH} n^{\prime}$ denotes the Baker-Campbell-Hausdorff series in the Lie algebra $\mathfrak{n}$, which is finite since $\mathfrak{n}$ is nilpotent.

Definition 2.32. Let $\left\{H_{j}\right\}_{j=1}^{n}$ and $\left\{N_{j}\right\}_{j=1}^{m}$ be bases of $\mathfrak{a}$ and $\mathfrak{n}$ respectively. The coordinates system

$$
\begin{aligned}
\mathbb{R}^{n+m} \rightarrow \mathfrak{a} \oplus \mathfrak{n}, \quad & \left(a_{1}, \ldots, a_{n}, n_{1}, \ldots, n_{m}\right) \mapsto \\
& \left(\operatorname{arcsinh}\left(a_{1}\right) H_{1}+\cdots+\operatorname{arcsinh}\left(a_{n}\right) H_{n}, n_{1} N_{1}+\cdots+n_{m} N_{m}\right),
\end{aligned}
$$

are said to be adapted tempered coordinates for $\mathbb{B}$.

LEMMA 2.33. In any adapted tempered coordinates on $\mathbb{B}$, the multiplication and inverse operations are tempered maps $\mathbb{R}^{n+m} \times \mathbb{R}^{n+m} \rightarrow \mathbb{R}^{n+m}$ and $\mathbb{R}^{n+m} \rightarrow \mathbb{R}^{n+m}$ respectively.

Proof. Let $a_{1}, \ldots, a_{n}, n_{1}, \ldots, n_{m}$ be adapted tempered coordinates on $\mathbb{B}$ as in the above definition. Then, since

$$
\sinh \left(a+a^{\prime}\right)=\sinh a \cosh a^{\prime}+\cosh a \sinh a^{\prime},
$$

the $\left\{a_{i}\right\}$-coordinates of the multiplication of $x, x^{\prime} \in \mathbb{R}^{n+m}$ read

$$
\sinh \left(\operatorname{arcsinh} a_{i}+\operatorname{arcsinh} a_{i}^{\prime}\right)=a_{i} \sqrt{1+{a_{i}^{\prime 2}}^{2}}+a_{i}^{\prime} \sqrt{1+a_{i}^{2}},
$$

so that they clearly are tempered functions in the $a_{i}, a_{i}^{\prime}$ variables. For the $\mathfrak{n}$ part, recall that there is a decomposition in real root spaces $\mathfrak{n}=\bigoplus_{\alpha} \mathfrak{n}_{\alpha}$ for the adjoint action of $\mathfrak{a}$. Now if $n^{\prime} \in \mathfrak{n}_{\alpha}$, we have

$$
\begin{aligned}
& e^{\operatorname{ad}\left(\operatorname{arcsinh}\left(a_{1}\right) H_{1}+\cdots+\operatorname{arcsinh}\left(a_{n}\right) H_{n}\right)} n^{\prime}=e^{\alpha\left(H_{1}\right) \operatorname{arcsinh}\left(a_{1}\right)+\cdots+\alpha\left(H_{2}\right) \operatorname{arcsinh}\left(a_{n}\right)} n^{\prime} \\
& =\left(a_{1}+\sqrt{1+a_{1}^{2}}\right)^{\alpha\left(H_{1}\right)} \ldots\left(a_{n}+\sqrt{1+a_{n}^{2}}\right)^{\alpha\left(H_{n}\right)} n^{\prime},
\end{aligned}
$$

which is a tempered function in $a_{1}, \ldots, a_{n}$. As the $\mathrm{CBH}$ product in a nilpotent group is polynomial, linearly decomposing $n_{1}^{\prime} N_{1}+\ldots n_{m}^{\prime} N_{m}$ along the root space decomposition and using the above computation, we get that the $n_{i}$ coordinates of the product of $x$ and $x^{\prime}$ are tempered in all variables. For the inverse map, as $(a, n)^{-1}=\left(-a,-e^{-\mathrm{ad} a} n\right)$, the above computation also shows the result.

Lemma 2.34. Let $\mathfrak{b}=\mathfrak{b}^{\prime} \ltimes \mathfrak{s}$ be a Pyatetskii-Shapiro decomposition of a normal $\mathbf{j}$ algebra $\mathfrak{b}$, with $\mathfrak{s}$ an elementary normal $\mathbf{j}$-algebra and with corresponding Lie group decomposition $\mathbb{B}=\mathbb{B}^{\prime} \ltimes \mathbb{S}$. Denote $\mathbf{R}: \mathbb{B}^{\prime} \rightarrow \operatorname{Aut}(\mathbb{S})$ the associated extension homomorphism. Then in any adapted tempered coordinates for $\mathbb{B}^{\prime}=\mathfrak{a}^{\prime} \oplus \mathfrak{n}^{\prime}$, with 
$\operatorname{dim}\left(\mathfrak{a}^{\prime}\right)=n^{\prime}, \operatorname{dim}\left(\mathfrak{n}^{\prime}\right)=m^{\prime}$ and $\mathbb{S}=\mathfrak{a} \oplus \mathfrak{n}$ (recall that by Proposition 2.5, $\mathfrak{n}$ is an Heisenberg Lie algebra, thus nilpotent), with $\operatorname{dim}(\mathfrak{a})=1, \operatorname{dim}(\mathfrak{n})=m, \mathbf{R}$ is a tempered map $\mathbb{R}^{n^{\prime}+m^{\prime}} \times \mathbb{R}^{1+m} \rightarrow \mathbb{R}^{1+m}$.

Proof. Let $a_{1}, \ldots, a_{n^{\prime}}, n_{1}, \ldots, n_{m^{\prime}}$ and $a_{n^{\prime}+1}, n_{m^{\prime}+1}, \ldots, n_{m^{\prime}+m_{1}}$ be adapted tempered coordinates for $\mathbb{B}^{\prime}$ and $\mathbb{S}$ respectively. The group $\mathbb{B}^{\prime}$ acts trivially on $H_{n^{\prime}+1}$, the generator of $\mathfrak{a}$. Moreover, the coordinates $a_{1}, \ldots, a_{n^{\prime}+1}, n_{1}, \ldots, n_{m^{\prime}+m_{1}}$ are adapted tempered coordinates for $\mathbb{B}$. Indeed, one knows [22, pages 56-57] that the infinitesimal action of $H_{1}, \ldots, H_{n^{\prime}}$ is real semi-simple with spectrum contained in $\left\{-\frac{1}{2}, 0, \frac{1}{2}\right\}$. Denote $i^{\prime}: \mathbb{B}^{\prime} \rightarrow \mathbb{B}$ and $i: \mathbb{S} \rightarrow \mathbb{B}$ the inclusions seen through the coordinates. Now by Lemma 2.33 the map

$$
\left(x^{\prime}, x\right) \in \mathbb{B}^{\prime} \times \mathbb{S} \mapsto i^{\prime}\left(x^{\prime}\right) \cdot i(x) \in \mathbb{B},
$$

is tempered. But the $\mathfrak{n}$ part of that product is exactly $\mathbf{R}_{x^{\prime}}(x)$ and so, this concludes the proof.

We are now prepared to state and prove the main result of this chapter.

Theorem 2.35. Let $\mathbb{B}$ be a normal $\mathbf{j}$-group with Pyatetskii-Shapiro decomposition $\mathbb{B}=\left(\mathbb{S}_{N} \ltimes \ldots\right) \ltimes \mathbb{S}_{1}$. Parametrizing the elements $g, g^{\prime} \in \mathbb{B}$ as $g=g_{1} g_{2} \ldots g_{N}$ and $g^{\prime}=g_{1}^{\prime} g_{2}^{\prime} \ldots g_{N}^{\prime}$ with $g_{i}, g_{i}^{\prime} \in \mathbb{S}_{i}$, we define

$$
S_{\text {can }}^{\mathbb{B}}: \mathbb{B} \times \mathbb{B} \rightarrow \mathbb{R}, \quad\left(g, g^{\prime}\right) \mapsto \sum_{i=1}^{N} S_{\text {can }}^{\mathbb{S}_{i}}\left(g_{i}, g_{i}^{\prime}\right),
$$

where $S_{\text {can }}^{\mathbb{S}_{i}}$ is the canonical phase of $\mathbb{S}_{i}$ given in Definition 2.15. Then the pair $\left(\mathbb{B} \times \mathbb{B}, S_{\text {can }}^{\mathbb{B}}\right)$ is tempered admissible and tame.

Proof. We will use an induction over $N$, the number of elementary factors in $\mathbb{B}$. Accordingly, we set $\mathbb{B}=\mathbb{B}^{\prime} \ltimes_{\mathbf{R}} \mathbb{S}$, with $\mathbb{B}^{\prime}:=\left(\mathbb{S}_{N} \ltimes \ldots\right) \ltimes \mathbb{S}_{2}$ and $\mathbb{S}:=\mathbb{S}_{1}$. We then observe that $\mathbb{B} \times \mathbb{B}=\left(\mathbb{B}^{\prime} \times \mathbb{B}^{\prime}\right) \ltimes \mathbf{R} \times \mathbf{R}(\mathbb{S} \times \mathbb{S})$ and from Lemma 1.5 and Lemma 2.34, that the extension homomorphism $\mathbf{R} \times \mathbf{R}=: \mathbf{R}^{2}$ is tempered within adapted coordinates. By Theorem 2.29, the pair $\left(\mathbb{S} \times \mathbb{S}, S_{\mathrm{can}}^{\mathbb{S}}\right)$ is tempered and admissible. By induction hypothesis, the latter also holds for $\left(\mathbb{B}^{\prime} \times \mathbb{B}^{\prime}, S_{\text {can }}^{\mathbb{B}^{\prime}}\right)$. Moreover, Equations (2.14) tell that, in the "elementary" case of $\mathbb{S} \times \mathbb{S}$, the adapted tempered coordinates and the coordinates associated to the phase function are related to one another through a tempered diffeomorphism. By induction hypothesis, the latter also holds for $\mathbb{B}^{\prime} \times \mathbb{B}^{\prime}$. Obviously, the extension homomorphism $\mathbf{R} \times \mathbf{R}$ is then tempered within the coordinates associated to the phase functions as well. Note that under the parametrization $g=g_{1} g^{\prime}, h=h_{1} h^{\prime} \in \mathbb{B}^{\prime}, g_{1}, h_{1} \in \mathbb{S}_{1}, g^{\prime}, h^{\prime} \in \mathbb{B}^{\prime} \in \mathbb{B}^{\prime}$, the multiplication and inverse maps of $\mathbb{B}$ become:

$$
g h=g_{1} \mathbf{R}_{g^{\prime}}\left(h_{1}\right) g^{\prime} h^{\prime}, \quad g^{-1}=\mathbf{R}_{g^{\prime-1}}\left(g_{1}^{-1}\right) g^{\prime-1},
$$

and similarly for $\mathbb{B} \times \mathbb{B}$. From this and the temperedness of the extension homomorphism $\mathbf{R} \times \mathbf{R}$, we see that temperedness of the multiplication and inversion laws in $\mathbb{B} \times \mathbb{B}$ will immediately follow once we will have shown that the map (1.12) is a global diffeomorphism from $\mathbb{B} \times \mathbb{B}$ to $(\mathfrak{b} \oplus \mathfrak{b})^{\star}$. We will return to this question while examining admissibility. To this aim, let us set $G_{1}:=\mathbb{B}^{\prime} \times \mathbb{B}^{\prime}, G_{2}:=\mathbb{S} \times \mathbb{S}$ and denote respectively by $\mathfrak{g}_{1}$ and $\mathfrak{g}_{2}$ their Lie algebras. Let us also set $S_{1}:=S_{\text {can }}^{\mathbb{B}^{\prime}}, S_{2}:=S_{\text {can }}^{\mathbb{S}}$, and let us assume, by induction hypothesis, that the pair $\left(G_{1}, S_{1}\right)$ is admissible, with associated decomposition $\mathfrak{g}_{1}=\oplus_{k=0}^{N_{1}} \mathfrak{W}_{k}$. Let us consider an adapted basis 
of $\mathfrak{g}_{1},\left\{{ }_{1} w_{k}\right\}, k=1, \ldots, \operatorname{dim}\left(\mathfrak{g}_{1}\right)$ with associated coordinates ${ }_{1}(b)_{k}:=\widetilde{{ }_{1} w_{k}} \cdot S_{1}(b)$ on $G_{1}$. Similarly, let us consider the basis $\left\{{ }_{2} w_{j}^{r} \mid r=1,2 ; j=0,1,2,3\right\}$ of $\mathfrak{g}_{2}$ adapted to the decomposition (2.11), where, for the values 1 and $2, j$ consists of a multi-index and where $r$ labels the copies of $\mathbb{S}$ in $\mathbb{S} \times \mathbb{S}$. Accordingly, we have the associated coordinate system (2.14) on $G_{2}$ that now reads ${ }_{2}(x)_{j}^{r}:={ }_{2} w_{j}^{r} \cdot S_{2}(x)$.

On $G:=G_{1} \ltimes_{\mathbf{R}^{2}} G_{2}$, with $S(x b):=S_{1}(b)+S_{2}(x), x \in G_{2}, b \in G_{1}$, we then compute that:

$$
{ }_{1}(x b)_{k}:=\widetilde{{ }_{1} w_{k}} \cdot S(x b)={ }_{1}(b)_{k} \quad \text { and } \quad{ }_{2}(x b)_{j}^{r}:=\widetilde{{ }_{2} w_{j}^{r}} \cdot S(x b)=\widetilde{\mathbf{R}_{b}^{2}\left({ }_{2} w_{j}^{r}\right)} \cdot S_{2}(x) .
$$

Hence it suffices to look at the properties of the multipliers within $\mathfrak{g}_{2}$. From Pyatetskii-Shapiro's theory, we know that for the values 0 and 3 of $j$, the action of $G_{1}$ is trivial: $\mathbf{R}_{b}^{2}\left({ }_{2} w_{j}^{r}\right)={ }_{2} w_{j}^{r}$. Hence:

$$
{ }_{2}(x b)_{j}^{r}={ }_{2}(x)_{j}^{r}, \quad \forall j \in\{0,3\} .
$$

Hence it suffices to look at the properties of the multipliers within the subspace $V \times V=\mathfrak{V}_{1} \oplus \mathfrak{V}_{2}$. For $j=1,2$, however, the action is not trivial but stabilizes component-wise $V \times V$. Accordingly, we set:

$$
\mathbf{R}_{b}^{2}\left({ }_{2} w_{j}^{r}\right)=: \sum_{p=1}^{2}\left[\mathbf{R}_{b}^{2}\right]_{j 2}^{p} w_{p}^{r}, \quad \forall j \in\{1,2\},
$$

where, again, $p$ is a multi-index. We therefore have:

$$
{ }_{2}(x b)_{j}^{r}=\sum_{p=1}^{2}\left[\mathbf{R}_{b}^{2}\right]_{j 2}^{p}(x)_{p}^{r}, \quad \forall j \in\{1,2\} .
$$

From Pyatetskii-Shapiro's theory, we know that the linear operator $\left[\mathbf{R}_{b}^{2}\right]_{j}^{p}$ is symplectic, thus it has jacobian one. In particular, this implies that the map (1.12) is a global diffeomorphism from $G$ to $\mathfrak{g}^{\star}$. (Hence, we have completed the proof of temperedness of the pair $\left(\mathbb{B} \times \mathbb{B}, S_{\text {can }}^{\mathbb{B}}\right)$.) We now consider the ordered decomposition:

$$
\mathfrak{g}=\mathfrak{g}_{2} \oplus \mathfrak{g}_{1}=\left(\oplus_{j=0}^{3} \mathfrak{V}_{j}\right) \bigoplus\left(\oplus_{k=0}^{N_{1}} \mathfrak{W}_{k}\right),
$$

where indices occurring on the left $\left(\mathfrak{g}_{2}\right)$ are considered as lower than the one on the right $\left(\mathfrak{g}_{1}\right)$. Within this setting, we compute that for every element $X \in \mathcal{U}\left(\mathfrak{g}_{2}\right)$ :

$$
{ }_{(2)} \alpha_{X}(x b):=e^{-i S(x b)}\left(\widetilde{X} e^{i S}\right)(x b)={ }_{2} \alpha_{\mathbf{R}_{b}^{2}(X)}(x),
$$

where ${ }_{2} \alpha_{X}:=e^{-i S_{2}}\left(\widetilde{X} \cdot e^{i S_{2}}\right)$ denotes the multiplier on $G_{2}$. Again, for the extreme values of $j$, we observe that:

$$
{ }_{(2)} \alpha_{X}(x b)={ }_{2} \alpha_{X}(x), \quad \forall X \in \mathcal{U}\left(\mathfrak{V}_{0} \oplus \mathfrak{V}_{3}\right),
$$

so in these cases the properties underlying admissibility are trivially satisfied. For $j=1,2$, we have with the notation $X_{j}:=1-\sum_{r=1}^{2}\left({ }_{2} w_{j}^{r}\right)^{2}$ of Proposition 2.26

$$
\mathbf{R}_{b}^{2}\left(X_{j}\right)=1-\sum_{r=1}^{2}\left(\left[\mathbf{R}_{b}^{2}\right]_{j}^{p} w_{p}^{r}\right)^{2}
$$

which leads to

$$
{ }_{(2)} \alpha_{X_{j}}(x b)=1+\sum_{r=1}^{2}\left(2(x b)_{j}^{r}\right)^{2}-i \sum_{r=1}^{2}\left[\mathbf{R}_{b_{r}}\right]_{j}^{p_{r}}\left[\mathbf{R}_{b_{r}}\right]_{j}^{p_{r}^{\prime}} \widetilde{w_{p_{r}}^{r} \cdot 2}(x)_{p_{r}^{\prime}}^{r},
$$


where $b=\left(b_{1}, b_{2}\right) \in \mathbb{B}^{\prime} \times \mathbb{B}^{\prime}=G_{1}$. This gives the property $(i)$ of Definition 1.24 From the expression (2.16) and the structure of the elementary case (Lemma 2.20), we then observe:

$$
\widetilde{A}_{(2)} \alpha_{X_{j}}=0, \quad \forall A \in \mathcal{U}(V \times V), \operatorname{deg}(A) \geq 3 .
$$

Also, setting $-i \beta_{X_{j}}(x b):=-i \sum_{r=1}^{2}\left[\mathbf{R}_{b_{r}}\right]_{j}^{p_{r}}\left[\mathbf{R}_{b_{r}}\right]_{j}^{p_{r}^{\prime}} \widetilde{w_{p_{r}}^{r} \cdot 2}(x)_{p_{r}^{\prime}}^{r}$, we deduce from the expressions (2.8) and (2.9) that, for every $A \in V \times V: \widetilde{A} \cdot \beta_{X_{j}}=0$. From the expression (2.16) and setting ${ }_{2}(x)_{12}:=\left({ }_{2}(x)_{1},{ }_{2}(x)_{2}\right)$, we then deduce that for every $A \in \mathcal{U}(V \times V)$ of strictly positive degree:

$$
\begin{aligned}
\left|\widetilde{A}\left(_{(2)} \alpha_{X_{1}}(x b)+{ }_{(2)} \alpha_{X_{2}}(x b)\right)\right|=\left.|\widetilde{A}|_{2}(x b)_{12}\right|^{2} \mid & =\left.\left|\widetilde{\mathbf{R}_{b}^{2}(A)}{ }_{x}\right| \mathbf{R}_{b}^{2}\left(_{2}(x)_{12}\right)\right|^{2} \mid \\
& \leq\left|\mathbf{R}_{b}^{2}\right|^{\operatorname{deg}(A)+2} C_{A} \mu_{12}(x)\left|\left({ }_{2}(x)_{12}\right)\right|^{2}
\end{aligned}
$$

where the last estimate is obtained from Corollary 2.24. Since

$$
\left|\left({ }_{2}(x)_{12}\right)\right|^{2}=\left|\mathbf{R}_{b^{-1}}^{2} \mathbf{R}_{b}^{2}\left({ }_{2}(x)_{12}\right)\right|^{2} \leq\left|\mathbf{R}_{b^{-1}}^{2}\right|^{2}\left|\left({ }_{2}(x b)_{12}\right)\right|^{2},
$$

we then get

$$
\begin{aligned}
\mid \widetilde{A}\left(_{(2)} \alpha_{X_{1}}(x b)\right. & \left.\left.+{ }_{(2)} \alpha_{X_{2}}(x b)\right)\left.|\leq| \mathbf{R}_{b}^{2}\right|^{\operatorname{deg}(A)+2} C_{A} \mu_{12}(x)\left|\mathbf{R}_{b^{-1}}^{2}\right|^{2} \mid{ }_{2}(x b)_{12}\right)\left.\right|^{2} \\
& \leq C_{A}\left|\mathbf{R}_{b}^{2}\right|^{\operatorname{deg}(A)+2}\left|\mathbf{R}_{b^{-1}}^{2}\right|^{2} \mu_{12}(x)\left|{ }_{(2)} \alpha_{X_{1}}(x b)+{ }_{(2)} \alpha_{X_{2}}(x b)\right| .
\end{aligned}
$$

But by (2.17), we know that we may assume $\operatorname{deg}(A) \leq 2$, hence

$$
\left|\widetilde{A}\left({ }_{(2)} \alpha_{X_{1}}(x b)+{ }_{(2)} \alpha_{X_{2}}(x b)\right)\right| \leq C_{A} \mathfrak{d}_{G}^{6}(b) \mu_{12}(x)\left|{ }_{(2)} \alpha_{X_{1}}(x b)+{ }_{(2)} \alpha_{X_{2}}(x b)\right| .
$$

Defining the element $\mu_{12}^{\prime}(x b):=\mathfrak{d}_{G}^{6}(b) \mu_{12}(x)$ yields admissibility at the level of $V \times V$.

Last, tameness also follows by an induction argument, using Lemma 1.5 and Lemma 2.31

REMARK 2.36. Observe that Remarks 1.20. Lemma 1.21 and Lemma 2.31 show that on the one-variable Schwartz space $\mathcal{S}^{S_{\text {can }}^{\mathbb{B}}}(\mathbb{B}, \mathcal{E})$ associated with the two-variable tempered, admissible and tame pair $\left(\mathbb{B} \times \mathbb{B}, S_{\text {can }}^{\mathbb{B}}\right)$ (see Definition 1.48) one has the same topology associated with the equivalent semi-norms

$$
f \in \mathcal{S}^{S}(\mathbb{B}, \mathcal{E}) \mapsto \sup _{X \in \mathcal{U}_{k}(\mathfrak{b})} \sup _{x \in \mathbb{B}}\left\{\frac{\mathfrak{d}_{\mathbb{B}}(x)^{n}\|\tilde{X} f(x)\|_{j}}{|X|_{k}}\right\}, \quad j, k, n \in \mathbb{N},
$$

or even with

$$
f \in \mathcal{S}^{S}(\mathbb{B}, \mathcal{E}) \mapsto \sup _{X \in \mathcal{U}_{k}(\mathfrak{b})} \sup _{x \in \mathbb{B}}\left\{\frac{\mathfrak{d}_{\mathbb{B}}(x)^{n}\|\underline{X} f(x)\|_{j}}{|X|_{k}}\right\}, \quad j, k, n \in \mathbb{N} .
$$





\section{CHAPTER 3}

\section{Non-formal star-products}

This chapter is devoted to the construction of an infinite dimensional parameter family of non-formal star-products on every negatively curved Kählerian Lie group. In section 3.1] we first review the construction of one of us of such non-formal star-products, living on a space of distributions. Their covariance properties and their relations with the Moyal product are given there. In section 3.2, we apply the results of chapters 1 and 2 to give a proper interpretation of the star-product formula as an oscillatory integral and eventually prove that the Fréchet space $\mathcal{B}(\mathbb{B})$ becomes a Fréchet algebra for all these star-products. This is proved in Theorem 3.9, the main result of this chapter and, we believe, and important result in itself.

\subsection{Star-products on normal j-groups}

We consider an elementary normal $\mathbf{j}$-group $\mathbb{S}$ viewed as a symplectic symmetric spaces as in section 2.2. We start by recalling the results obtained in $[\mathbf{2}, \mathbf{3}$.

Definition 3.1. Set $\tilde{\mathbb{S}}:=\{(a, v, \xi)\}=\mathbb{R} \times \mathbb{R}^{2 d} \times \mathbb{R}$. The twisting map is the smooth one-parameter family of diffeomorphisms defined as

$$
\phi_{\theta}: \tilde{\mathbb{S}} \rightarrow \tilde{\mathbb{S}}:(a, v, \xi) \mapsto\left(a, \operatorname{sech}\left(\frac{\theta}{4} \xi\right) v, \frac{2}{\theta} \sinh \left(\frac{\theta}{2} \xi\right)\right), \quad \theta \in \mathbb{R}^{*} .
$$

Let $\mathcal{S}(\mathbb{S})$ be the Euclidean Schwartz space of $\mathbb{S}$, i.e. the ordinary Schwartz space in the coordinates (2.4). Accordingly, let $\mathcal{S}(\mathbb{S})^{\prime}$ be the dual space of tempered distributions. Let us also denote by

$$
(\mathcal{F} u)(a, v, \xi):=\int_{-\infty}^{\infty} e^{-i \xi t} u(a, v, t) \mathrm{d} t,
$$

the partial Fourier transform in the $t$-variable. For $\gamma>0$, we let $\mathcal{O}_{C, \gamma}\left(\mathbb{R}^{m}\right)$ be the subset of smooth functions, the derivatives of which are uniformly polynomially bounded:

$$
\begin{aligned}
& \mathcal{O}_{C, \gamma}\left(\mathbb{R}^{m}\right):= \\
& \left\{f \in C^{\infty}\left(\mathbb{R}^{m}\right): \exists r>0: \forall \alpha \in \mathbb{N}^{m}: \exists C_{\alpha}>0,\left|\partial^{\alpha} f(x)\right| \leq C_{\alpha}(1+|x|)^{r-\gamma|\alpha|}\right\} .
\end{aligned}
$$

Note that $\mathcal{O}_{C, 1}\left(\mathbb{R}^{m}\right)$ is the space of Grossmann-Loupias-Stein symbols, traditionally written $\mathcal{K}\left(\mathbb{R}^{m}\right)$.

Definition 3.2. We denote by $\boldsymbol{\Theta}$, the subspace of $C^{\infty}(\mathbb{R}, \mathbb{C})$ constituted by the elements $\tau$ such that $\exp \circ \pm \tau$ belong to the space $\mathcal{O}_{C, 1}(\mathbb{R}, \mathbb{C})$, and normalized such that $\tau(0)=0$.

Let $\tau_{0}$ be the element of $C^{\infty}(\tilde{\mathbb{S}})$, given by:

$$
\tau_{0}:=\frac{1}{2} \log \circ \mathrm{Jac}_{\phi_{\theta}^{-1}} .
$$


Viewed as a function of its last variable only, $\tau_{0}$ belongs to $\boldsymbol{\Theta}$. Indeed, we have:

$$
\operatorname{Jac}_{\phi_{\theta}^{-1}}(a, v, \xi)=2^{-d} \frac{\left(1+\sqrt{1+\frac{\theta^{2} \xi^{2}}{4}}\right)^{d}}{\sqrt{1+\frac{\theta^{2} \xi^{2}}{4}}} .
$$

To an element $\tau \in \boldsymbol{\Theta}$, one associates a function on $\tilde{\mathbb{S}}$ by $[(a, v, \xi) \mapsto \tau(\xi)]$ and, to simplify the notations, we still denote this function by $\tau$. One then defines a linear injection:

$$
T_{\theta, \tau}:=\mathcal{F}^{-1} \circ \exp \left(\tau_{0}-\tau\right) \circ\left(\phi_{\theta}^{-1}\right)^{\star} \circ \mathcal{F}: \mathcal{S}(\mathbb{S}) \rightarrow \mathcal{S}(\mathbb{S})^{\prime},
$$

where, by a slight abuse of notation, we identify a function with the linear operator of point-wise multiplication by this function. We make the following obvious but important observation:

Lemma 3.3. Let $\tau \in \Theta$. Then the inverse of the map $T_{\theta, \tau}$, given by

$$
T_{\theta, \tau}^{-1}=\mathcal{F}^{-1} \circ\left(\phi_{\theta}\right)^{\star} \circ \exp \left(-\tau_{0}+\tau\right) \circ \mathcal{F},
$$

defines a continuous linear injection from $\mathcal{S}(\mathbb{S})$ to itself. Moreover, $T_{\theta, \tau}^{-1}$ extends to a unitary operator on $L^{2}(\mathbb{S})$ if and only if $\tau$ is purely imaginary.

Proof. As the partial Fourier transform $\mathcal{F}$ is an homeomorphism from $\mathcal{S}(\mathbb{S})$ to $\mathcal{S}(\tilde{\mathbb{S}})$, and as $\exp \left(-\tau_{0}+\tau\right)$ belongs to $\mathcal{O}_{C, 1}(\mathbb{R}, \mathbb{C})$, a subspace of the Schwartz multipliers $\mathcal{O}_{M}(\mathbb{R}, \mathbb{C})$, it suffices to show that the map $f \mapsto f \circ \phi_{\theta}$, is continuous on $\mathcal{S}(\tilde{\mathbb{S}})$. So, let $f \in \mathcal{S}(\tilde{\mathbb{S}})$. Then, as

$$
f \circ \phi_{\theta}(a, v, \xi)=f\left(a, \operatorname{sech}\left(\frac{\theta}{4} \xi\right) v, \frac{2}{\theta} \sinh \left(\frac{\theta}{2} \xi\right)\right),
$$

we deduce from

$$
\partial_{a}^{k}\left(f \circ \phi_{\theta}\right)=\left(\partial_{a}^{k} f\right) \circ \phi_{\theta}, \quad \partial_{v}^{m}\left(f \circ \phi_{\theta}\right)=\operatorname{sech}\left(\frac{\theta}{4} \xi\right)^{m}\left(\partial_{v}^{m} f\right) \circ \phi_{\theta},
$$

and from an iterative use of the relation

$\partial_{\xi}\left(f \circ \phi_{\theta}\right)(a, v, \xi)=-\frac{\theta}{4} \frac{\sinh \left(\frac{\theta}{4} \xi\right)}{\cosh \left(\frac{\theta}{4} \xi\right)^{2}} v\left(\partial_{v} f\right) \circ \phi_{\theta}(a, v, \xi)+\cosh \left(\frac{\theta}{4} \xi\right)\left(\partial_{\xi} f\right) \circ \phi_{\theta}(a, v, \xi)$,

that any (ordinary) derivatives of $f \circ \phi_{\theta}$ is a finite linear combination of derivatives of $f$ composed with $\phi_{\theta}$ and with coefficient in the polynomial ring $\mathbb{R}\left[v, e^{\theta \xi}, e^{-\theta \xi}\right]$. To conclude the first claim, we then observe that as a derivative of a Schwartz function is a Schwartz function, any derivatives of $f$ composed with $\phi_{\theta}$ is bounded (in absolute value) by $\left(a^{2}+|v|^{2}+\cosh (\theta \xi)\right)^{-n}$, with $n$ arbitrary. For the second claim, observe that

$$
T_{\theta, \tau}^{-1}=\mathcal{F}^{-1} \circ\left(\phi_{\theta}\right)^{\star} \circ \operatorname{Jac}_{\phi_{\theta}^{-1}}^{-1 / 2} \circ \exp (\tau) \circ \mathcal{F}=\mathcal{F}^{-1} \circ \operatorname{Jac}_{\phi_{\theta}}^{1 / 2} \circ\left(\phi_{\theta}\right)^{\star} \circ \exp (\tau) \circ \mathcal{F},
$$

which entails since $\mathcal{F}$ and $\operatorname{Jac}_{\phi_{\theta}}^{1 / 2} \circ\left(\phi_{\theta}\right)^{\star}$ are unitary, that $T_{\theta, \tau}^{-1}$ is unitary if and only if the operator of multiplication by $\exp (\tau)$ is unitary, which is equivalent to $\Re(\tau)=0$.

REMark 3.4. Denoting by $T_{\theta, \tau}^{*}$ the formal adjoint of $T_{\theta, \tau}$ with respect to the inner product of $L^{2}(\mathbb{S})$, then we have $T_{\theta, \tau}^{*}=T_{\theta,-\bar{\tau}}^{-1}$ and thus, Lemma (3.3) implies that $T_{\theta, \tau}^{*}$ is continuous on $\mathcal{S}(\mathbb{S})$ too. 
Let $\omega^{0}$ be the standard symplectic structure of $\mathbb{R}^{2 d+2} \simeq T^{*} \mathbb{R}^{d+1}$ and let $\star_{\theta}^{0}$ be the Moyal product in its integral form on $\mathcal{S}\left(\mathbb{R}^{2 d+2}\right)$. Recall that the latter product is by definition the composition law of symbols in the Weyl pseudo-differential calculus and that it is given by

$$
f_{1} \star_{\theta}^{0} f_{2}(x)=\frac{1}{(\pi \theta)^{2(d+1)}} \int_{\mathbb{R}^{2 d+2} \times \mathbb{R}^{2 d+2}} e^{\frac{2 i}{\theta} S_{0}(x, y, z)} f_{1}(y) f_{2}(z) \mathrm{d} y \mathrm{~d} z,
$$

where $S_{0}(x, y, z):=\omega^{0}(x, y)+\omega^{0}(y, z)+\omega^{0}(z, x)$. For $\tau \in \boldsymbol{\Theta}$, denoting by

$$
\mathcal{E}_{\theta, \tau}(\mathbb{S}):=T_{\theta, \tau}(\mathcal{S}(\mathbb{S}))
$$

the range subspace of $T_{\theta, \tau}$ in the tempered distribution space $\mathcal{S}(\mathbb{S})^{\prime}$, one has the inclusions

$$
\mathcal{S}(\mathbb{S}) \subset \mathcal{E}_{\theta, \tau}(\mathbb{S}) \subset C^{\infty}(\mathbb{S})
$$

We consider the linear isomorphism:

$$
T_{\theta, \tau}^{-1}: \mathcal{E}_{\theta, \tau}(\mathbb{S}) \rightarrow \mathcal{S}(\mathbb{S})
$$

Identifying $\mathbb{S} \simeq \mathbb{R}^{2 d+2}$ by means of the global coordinate system (2.4), we transport under $T_{\theta, \tau}$ the Moyal product on $\mathcal{S}\left(\mathbb{R}^{2 d+2}\right) \simeq \mathcal{S}(\mathbb{S})$. This yields an associative product:

$$
\star_{\theta, \tau}: \mathcal{E}_{\theta, \tau}(\mathbb{S}) \times \mathcal{E}_{\theta, \tau}(\mathbb{S}) \rightarrow \mathcal{E}_{\theta, \tau}(\mathbb{S})
$$

given by

$$
f_{1} \star_{\theta, \tau} f_{2}:=T_{\theta, \tau}\left(T_{\theta, \tau}^{-1}\left(f_{1}\right) \star_{\theta}^{0} T_{\theta, \tau}^{-1}\left(f_{2}\right)\right), \quad f_{1}, f_{2} \in \mathcal{E}_{\theta, \tau}(\mathbb{S}) .
$$

The associative algebra $\left(\mathcal{E}_{\theta, \tau}(\mathbb{S}), \star_{\theta, \tau}\right)$, endowed with the Fréchet algebra structure transported under $T_{\theta, \tau}$ from $\mathcal{S}\left(\mathbb{R}^{2 d+2}\right)$, satisfies the following properties [2,8]:

Theorem 3.5. Let $\tau \in \boldsymbol{\Theta}$ and $\theta \neq 0$. Then,

(i) For all compactly supported $u, v \in \mathcal{E}_{\theta, \tau}(\mathbb{S})$, one has the integral representation:

$$
u \star_{\theta, \tau} v=\int_{\mathbb{S} \times \mathbb{S}} K_{\theta, \tau}\left(x_{1}, x_{2}\right) R_{x_{1}}^{\star}(u) R_{x_{2}}^{\star}(v) \mathrm{d}_{\mathbb{S}}\left(x_{1}\right) \mathrm{d}_{\mathbb{S}}\left(x_{2}\right),
$$

where the two-point kernel is given by

$$
K_{\theta, \tau}\left(x_{1}, x_{2}\right):=(\pi \theta)^{-2(d+1)} A_{\theta, \tau}\left(x_{1}, x_{2}\right) \exp \left\{\frac{2 i}{\theta} S_{\text {can }}^{\mathbb{S}}\left(x_{1}, x_{2}\right)\right\},
$$

with, in the coordinated (2.4):

$A_{\theta, \tau}\left(x_{1}, x_{2}\right):=$

$A_{\text {can }}^{\mathbb{S}}\left(x_{1}, x_{2}\right) \exp \left\{\tau\left(\frac{2}{\theta} \sinh 2 a_{1}\right)+\tau\left(-\frac{2}{\theta} \sinh 2 a_{2}\right)-\tau\left(\frac{2}{\theta} \sinh \left(2 a_{1}-2 a_{2}\right)\right)\right\}$, and with $S_{\mathrm{can}}^{\mathbb{S}}$ and $A_{\mathrm{can}}^{\mathbb{S}}$ defined in (2.6) and (2.7).

(ii) The product $\star_{\theta, \tau}$ is equivariant under the automorphism group of the symplectic symmetric space $\left(\mathbb{S}, s, \omega^{\mathbb{S}}\right)$ : for all elements $g$ of $\operatorname{Aut}\left(\mathbb{S}, s, \omega^{\mathbb{S}}\right)$ and $u, v \in \mathcal{D}(\mathbb{S})$, one has

$$
g^{\star}(u) \star_{\theta, \tau} g^{\star}(v)=g^{\star}\left(u \star_{\theta, \tau} v\right) .
$$

\footnotetext{
${ }^{1}$ As usual, we set $x_{j}=\left(a_{j}, v_{j}, t_{j}\right) \in \mathbb{R}^{2 d+2}$
} 
REMARK 3.6. Observe that when $\Im(\tau) \neq 0$, then the amplitude $A_{\theta, \tau}$ also contribute to the phase of $K_{\theta, \tau}$. However, as the amplitude is a function of the variables in $\mathfrak{a} \times \mathfrak{a}:=\mathbb{R} H \times \mathbb{R} H$ only, in the two dimensional case (i.e. $V=\{0\}$ ), there is no $\tau \in \Theta$ such that the combined phase coincides with those found in [16]. In particular, this means that the upper half-plane is a symplectic manifold that supports two non-isomorphic Moyal quantizations.

Consider a normal $\mathbf{j}$-group decomposed, following Proposition 2.5 into a semidirect product $\mathbb{B}=\mathbb{B}^{\prime} \ltimes \mathbb{S}$ where $\mathbb{S}$ is elementary. One knows from Proposition 2.5 and 3 that the extension homomorphism $\mathbf{R}: \mathbb{B}^{\prime} \rightarrow \operatorname{Aut}(\mathbb{S})$ underlies a homomorphism from $\mathbb{B}^{\prime}$ into the isotropy subgroup $\operatorname{Aut}\left(\mathbb{S}, s, \omega^{\mathbb{S}}\right)_{e}$ at the unit element $e$ of $\mathbb{S}$ viewed as a symmetric space:

$$
\mathbf{R}: \mathbb{B}^{\prime} \rightarrow \operatorname{Sp}\left(V, \omega^{0}\right) \subset \operatorname{Aut}\left(\mathbb{S}, s, \omega^{\mathbb{S}}\right)_{e},
$$

where $\left(V, \omega^{0}\right)$ is the symplectic vector space attached to $\mathbb{S}$. In particular, the action of $\mathbb{B}^{\prime}$ leaves invariant the two-point kernel $K_{\theta, \tau}$ on $\mathbb{S} \times \mathbb{S}$. Iterating the above observation at the level of $\mathbb{B}^{\prime}$ and translating the "extension Lemma" in [6] within the present framework, we obtain:

Proposition 3.7. Let $\mathbb{B}$ be a normal $\mathbf{j}$-group with Pyatetskii-Shapiro decomposition $\mathbb{B}=\left(\mathbb{S}_{N} \ltimes \ldots\right) \ltimes \mathbb{S}_{1}$ and fix $\vec{\tau}:=\left(\tau_{1}, \ldots, \tau_{N}\right) \in \mathbf{\Theta}^{N}$. Parametrizing a group element $g \in \mathbb{B}$ as $g=g_{1} \ldots g_{N}$, with $g_{i} \in \mathbb{S}_{i}$, we consider the two-point kernel on $\mathbb{B}$ given by

$$
K_{\theta, \vec{\tau}}\left(g, g^{\prime}\right):=K_{\theta, \tau_{1}}\left(g_{1}, g_{1}^{\prime}\right) \ldots K_{\theta, \tau_{N}}\left(g_{N}, g_{N}^{\prime}\right),
$$

where $K_{\theta, \tau_{i}}$ is the two-points kernel on $\mathbb{S}_{i} \times \mathbb{S}_{i}$, defined in (3.4). Then, the bilinear mapping

$$
\star_{\theta, \vec{\tau}}:=\left[(u, v) \mapsto \int_{\mathbb{B} \times \mathbb{B}} K_{\theta, \vec{\tau}}\left(g, g^{\prime}\right) R_{g}^{\star}(u) R_{g^{\prime}}^{\star}(v) \mathrm{d}_{\mathbb{B}}(g) \mathrm{d}_{\mathbb{B}}\left(g^{\prime}\right)\right],
$$

is associative on

$$
\mathcal{E}_{\theta, \vec{\tau}}(\mathbb{B}):=\mathcal{E}_{\theta, \tau_{N}}\left(\mathbb{S}_{N}\right) \otimes \cdots \otimes \mathcal{E}_{\theta, \tau_{1}}\left(\mathbb{S}_{1}\right)
$$

(recall that $\mathcal{E}_{\theta, \tau_{j}}\left(\mathbb{S}_{j}\right)$ is nuclear). Moreover, at the level of compactly supported functions, the product $\star_{\theta, \vec{\tau}}$ is equivariant under the left-translations in $\mathbb{B}$.

\subsection{An oscillatory integral formula for the star-product}

In this section, we fix $\mathbb{B}$ a normal $\mathbf{j}$-group, with Lie algebra $\mathfrak{b}$. We also let $\vec{\tau} \in \boldsymbol{\Theta}^{N}$ be as above $(N$ is the number of elementary components in $\mathbb{B})$ and form the two-point kernel $K_{\theta, \vec{\tau}}$ on $\mathbb{B} \times \mathbb{B}$, defined in $(3.5)$. Proposition 3.7 implies that the deformed product

$$
u \star_{\theta, \vec{\tau}} v=\int_{\mathbb{B} \times \mathbb{B}} K_{\theta, \vec{\tau}}\left(g, g^{\prime}\right) R_{g}^{\star}(u) R_{g^{\prime}}^{\star}(v) \mathrm{d}_{\mathbb{B}}(g) \mathrm{d}_{\mathbb{B}}\left(g^{\prime}\right),
$$

is weakly associative (in the sense of Definition 1.46) and left $\mathbb{B}$-equivariant. The results of chapter 1 will allow to properly understand the integral in (3.6) as an oscillatory one. As a consequence, we will see that the deformed product extends as a continuous bilinear and associative map on the function space $\mathcal{B}(\mathbb{B}, \mathcal{A})$, for $\mathcal{A}$ a Fréchet algebra. We start with a simple fact: 
Lemma 3.8. Let $\mathbb{B}$ be an elementary normal $\mathbf{j}$-group and $\vec{\tau} \in \mathbf{\Theta}^{N}$. Then the amplitude $A_{\theta, \vec{\tau}}$, as given in Proposition 3.7, consists of an element of $\mathcal{B}^{\mu_{\tau}}(\mathbb{B} \times \mathbb{B})$ for a tempered weight $\mu_{\tau}$.

Proof. Consider first the case where $\mathbb{B}=\mathbb{S}$ is elementary. Within the notations of section 2.3, we have

$$
\left|\vec{x}_{3}\right|=\left|\left(x_{1,3}, x_{2,3}\right)\right|=\left|\left(-\sinh 2 a_{2}, \sinh 2 a_{1}\right)\right|=\left(\sinh ^{2} 2 a_{2}+\sinh ^{2} 2 a_{1}\right)^{1 / 2},
$$

so that the function

$$
\mu_{\text {can }}\left(x_{1}, x_{2}\right):=\cosh a_{1} \cosh a_{2},
$$

is a tempered weight. As the left invariant vector field $\widetilde{H}$ on $\mathbb{S}$ restricted to functions of depending on the variable $a$ only, coincides with the partial differentiation operator $\partial_{a}$, we get from the explicit expression

$A_{\text {can }}^{\mathbb{S}}\left(x_{1}, x_{2}\right)=\left(\cosh a_{1} \cosh a_{2} \cosh \left(a_{1}-a_{2}\right)\right)^{d} \sqrt{\cosh 2 a_{1} \cosh 2 a_{2} \cosh 2\left(a_{1}-a_{2}\right)}$, that there exists $\rho>0$ such that for any $X \in \mathcal{U}(\mathfrak{s} \oplus \mathfrak{s})$, there exists a constant $C_{X}>0$ with

$$
\left|\widetilde{X} A_{\text {can }}^{\mathbb{S}}\right| \leq C_{X} \mu_{\text {can }}^{\rho} .
$$

Hence $A_{\text {can }}^{\mathbb{S}} \in \mathcal{B}^{\mu_{\text {can }}^{\rho}}(\mathbb{S} \times \mathbb{S})$. Next, since $\tau \in \boldsymbol{\Theta}$, we have expo $\pm \tau \in \mathcal{O}_{C, 1}(\mathbb{R})$. Thus, there exists $r>0$ such that the $n$-th derivative of $\exp \circ \pm \tau(x)$ is bounded by $(1+|x|)^{r-n}$. Let us denote by $\operatorname{deg}(\tau)$ such positive number $r$. Since $\exp \circ \pm \tau$ depends on the variable $a$ only, among all elements of $\mathcal{U}(\mathfrak{s} \oplus \mathfrak{s})$, only the powers of $\widetilde{H}_{i}, i=1,2$, give non zero contributions. Therefore, an easy computation shows that for any $X \in \mathcal{U}(\mathfrak{s} \oplus \mathfrak{s})$, there exists a constant $C_{X}>0$ with

$$
\left|\widetilde{X} \exp \left\{ \pm \tau\left(\frac{2}{\theta} \sinh 2 a\right)\right\}\right| \leq C_{X}\left(1+\left|\vec{x}_{3}\right|\right)^{2 \operatorname{deg}(\tau)} .
$$

Hence $A_{\theta, \tau}$ belongs to $\mathcal{B}^{\mu_{\tau}}(\mathbb{S} \times \mathbb{S})$ for $\mu_{\tau}=\mu_{\text {can }}^{\rho+3 \operatorname{deg}(\tau)}$.

The general case $\mathbb{B}=\mathbb{B}^{\prime} \ltimes \mathbb{S}$ follows easily by Pyatetskii-Shapiro theory, since only the variables in $V \subset \mathbb{S}$ are affected by the action of $\mathbb{B}^{\prime}$ and that $A_{\theta, \vec{\tau}}$ is independent of these variables.

We now consider a Fréchet algebra $\mathcal{A}$, with topology underlying a countable family of sub-multiplicative semi-norms $\left\{\|\cdot\|_{j}\right\}_{j \in \mathbb{N}}$. Combining Lemma 3.8 with Theorem 2.35 leads us to prove that the integral in the expression of the deformed product (3.3) can be properly understood as an oscillatory one in the sense of chapter 1. In particular, this allows to define the product $\star_{\theta, \vec{\tau}}$ on $\mathcal{B}(\mathbb{B}, \mathcal{A})$. This is the main result of this chapter.

Before going further, a clarification regarding the notion of temperedness should be made. Recall that in the framework of a tempered pair $(G, S)$, the notion of temperedness comes from the global coordinate system associated to the phase function $S$ (see Definition 1.17 and Definition 1.22). Thus, in the case of a normal $\mathbf{j}$ group $\mathbb{B}$, this notion is a priori only defined on the product group $\mathbb{B} \times \mathbb{B}$. However, we have seen in the proof of Theorem 2.35 that the coordinate system (1.12) associated to the phase function $S_{\text {can }}^{\mathbb{B}}$ is related to the adapted tempered coordinates on $\mathbb{B} \times \mathbb{B}$ (see Definition 2.32) by a tempered diffeomorphism. Hence, it seems natural to define directly the notion of temperedness on $\mathbb{B}$ by mean of the adapted tempered coordinates. Having saying that, the important observation is that two functions $\varphi_{1}, \varphi_{2}$ are tempered on $\mathbb{B}$, if and only if $\varphi_{1} \otimes \varphi_{2}$ is tempered on $\mathbb{B} \times \mathbb{B}$. It implies a great simplification of the assumptions in Theorem 1.43, Proposition 1.47 and 
Proposition 1.50 namely the temperedness at the level of tensor product of weights can be reduced to temperedness at the level of each factor.

Theorem 3.9. Let $\mathbb{B}$ be a normal $\mathbf{j}$-group. Fix $\vec{\tau} \in \mathbf{\Theta}^{N}$ and let $\underline{\mu}_{1}, \underline{\mu}_{2}, \underline{\mu}_{3}$ be three families of tempered (in the sense of adapted tempered coordinates) weights on $\mathbb{B}$ of sub-multiplicativity degree $\left(\underline{L}_{1}, \underline{R}_{2}\right),\left(\underline{L}_{2}, \underline{R}_{2}\right),\left(\underline{L}_{3}, \underline{R}_{3}\right)$. Considering $K_{\theta, \vec{\tau}}$ the two-point kernel on $\mathbb{B}$ defined in (3.5), the correspondence

$$
\begin{aligned}
& \star_{\theta, \vec{\tau}}: \mathcal{B}^{\underline{\mu}}{ }_{1}(\mathbb{B}, \mathcal{A}) \times \mathcal{B}^{\mu_{2}}(\mathbb{B}, \mathcal{A}) \rightarrow \mathcal{B}^{\underline{\nu}}(\mathbb{B}, \mathcal{A}) \quad \text { with } \quad \nu_{j}=\mu_{1, j}^{L_{1, j}} \mu_{2, j}^{L_{2, j}} \\
& \left(F_{1}, F_{2}\right) \mapsto \int_{\mathbb{B} \times \mathbb{B}} K_{\theta, \vec{\tau}}\left[\left(x_{1}, x_{2}\right) \mapsto R_{x_{1}}^{\star}\left(F_{1}\right) R_{x_{2}}^{\star}\left(F_{2}\right)\right],
\end{aligned}
$$

is a continuous bilinear map and is equivariant under the left translations in $\mathbb{B}$ in the sense that for all $g \in \mathbb{B}$, we have

$L_{g}^{\star}\left(F_{1} \star_{\theta, \vec{\tau}} F_{2}\right)=\left(L_{g}^{\star} F_{1}\right) \star_{\theta, \vec{\tau}}\left(L_{g}^{\star} F_{2}\right) \quad$ in $\quad \mathcal{B} \underline{\lambda}(\mathbb{B}, \mathcal{A}) \quad$ for $\quad \underline{\lambda}=\left\{\mu_{1, j}^{L_{1, j} R_{1, j}} \mu_{2, j}^{L_{2, j} R_{2, j}}\right\}$. Moreover, the map $\star_{\theta, \vec{\tau}}$ is associative in the sense that then for every elements $F_{j}$ in $\mathcal{B}^{-}{ }^{\mu}(\mathbb{B}, \mathcal{A}), j=1,2,3$, we have the equality

$$
\left(F_{1} \star_{\theta, \vec{\tau}} F_{2}\right) \star_{\theta, \vec{\tau}} F_{3}=F_{1} \star_{\theta, \vec{\tau}}\left(F_{2} \star_{\theta, \vec{\tau}} F_{3}\right) \quad \text { in } \quad \mathcal{B} \underline{\rho}(\mathbb{B}, \mathcal{A}) \text {, }
$$

with

$$
\underline{\rho}=\left\{\mu_{1, j}^{L_{1, j}^{2}} \mu_{2, j}{ }^{L_{2, j}^{2}} \mu_{3, j}{ }^{L_{3, j}^{2}}\right\}_{j \in \mathbb{N}} .
$$

In particular, $\left(\mathcal{B}(\mathbb{B}, \mathcal{A}), \star_{\theta, \vec{\tau}}\right)$ is a Fréchet algebra.

Proof. That the bilinear map $\star_{\theta, \vec{\tau}}$ (with the domain and image as indicated) is well defined and continuous, follows from Theorem 1.43 (cf. the above discussion for the condition of temperedness of the weights involved), Theorem 2.35 and Lemma 3.8. Associativity follows from associativity in $\mathcal{E}_{\theta, \vec{\tau}}(\mathbb{B})$, which implies weak associativity in the sense of Definition 1.46 and Proposition 1.47. So, it remains to prove left $\mathbb{B}$-equivariance. We first note that by Lemma 1.12 (ii), the group $\mathbb{B}$ acts on the left continuously from $\mathcal{B} \underline{\mu}(\mathbb{B}, \mathcal{A})$ to $\mathcal{B} \underline{\underline{\gamma}}(\mathbb{B}, \mathcal{A})$, with $\underline{\gamma}=\left\{\mu_{j}^{R_{j}}\right\}$ (for any family of weights $\mu$ of sub-multiplicative degree $(\underline{L}, \underline{R})$ ). Also, we have by Lemma 1.44 that $F_{1} \star_{\theta, \vec{\tau}} F_{2}=\lim _{n_{1}, n_{2}} F_{1, n_{1}} \star_{\theta, \vec{\tau}} F_{2, n_{2}}$ in $\mathcal{B} \underline{\nu}(\mathbb{B}, \mathcal{A})$, with $\underline{\nu}=\left\{\mu_{1, j}^{L_{1, j}} \mu_{2, j}^{L_{2, j}}\right\}$, for any pair of sequences $\left\{F_{1, n}\right\}$ and $\left\{F_{2, n}\right\}$ of smooth compactly supported $\mathcal{A}$-valued functions on $\mathbb{B}$, which converge to $F_{1}$ and $F_{2}$, in the topology of $\mathcal{B} \underline{\hat{\mu}}_{1}(\mathbb{B}, \mathcal{A})$ and $\mathcal{B} \underline{\hat{\mu}}_{2}(\mathbb{B}, \mathcal{A})$ for any sequence of weights $\underline{\hat{\mu}}_{1}$ and $\underline{\hat{\mu}}_{2}$ dominating $\underline{\mu}_{1}$ and $\underline{\mu}_{2}$. From continuity of the left regular action (see Lemma $\overline{1.12}$ ii) and left $\bar{B}$-equivariance at the level of $\mathcal{D}(\mathbb{B}, \mathcal{A})$, we thus have

$$
L_{g}^{\star}\left(F \star_{\theta, \vec{\tau}} F^{\prime}\right)=\lim _{n, n^{\prime} \rightarrow \infty} L_{g}^{\star}\left(F_{n} \star_{\theta, \vec{\tau}} F_{n^{\prime}}^{\prime}\right)=\lim _{n, n^{\prime} \rightarrow \infty}\left(L_{g}^{\star} F_{n}\right) \star_{\theta, \vec{\tau}}\left(L_{g}^{\star} F_{n^{\prime}}^{\prime}\right),
$$

where the limits are in $\mathcal{B} \underline{\lambda}(\mathbb{B}, \mathcal{A})$, for $\underline{\lambda}=\left\{\mu_{1, j}^{L_{1, j} R_{1, j}} \mu_{2, j}^{L_{2, j} R_{2, j}}\right\}$. It remains to find specific approximation sequences $\left\{F_{1, n}\right\}$ and $\left\{F_{2, n}\right\}$, such that $\left\{L_{g}^{\star} F_{1, n}\right\}$ and $\left\{L_{g}^{\star} F_{2, n}\right\}$ converge to $L_{g}^{\star} F_{1}$ and $L_{g}^{\star} F_{2}$, in the topology of $\mathcal{B} \hat{\underline{\gamma}}_{1}(\mathbb{B}, \mathcal{A})$ and $\mathcal{B} \hat{\underline{\gamma}}_{2}(\mathbb{B}, \mathcal{A})$ with $\hat{\gamma}_{1}=\left\{\hat{\mu}_{1, j}^{R_{1, j}}\right\}$ and $\underline{\hat{\gamma}}_{2}=\left\{\hat{\mu}_{2, j}^{R_{2, j}}\right\}$. For this, we observe that the same construction as in the proof of Lemma 1.8 (viii), does the job. Indeed, recall that there, we have constructed the approximation sequence $\left\{F_{n}\right\}$, by setting for $F \in \mathcal{B}(\mathbb{B}, \mathcal{A})$ :

$$
F_{n}:=e_{n} F \in \mathcal{D}(\mathbb{B}, \mathcal{A}) \quad \text { where } \quad e_{n}:=\int_{\mathbb{B}} \psi(g) R_{g}^{\star}\left(\chi_{C_{n}}\right) \mathrm{d}_{\mathbb{B}}(g) \in \mathcal{D}(\mathbb{B}),
$$


and $0 \leq \psi \in \mathcal{D}(\mathbb{B}), \int_{\mathbb{B}} \psi(x) \mathrm{d}_{\mathbb{B}}(x)=1,\left\{C_{n}\right\}$ is an increasing sequence of relatively compact open subsets of $\mathbb{B}$ converging to $\mathbb{B}$ and $\chi_{C_{n}}$ is the characteristic function of $C_{n}$. Fixing $g \in \mathbb{B}$ and setting $C_{n}^{g}:=g \cdot C_{n}$, the sequence $\left\{C_{n}^{g}\right\}$ is still an increasing sequence of relatively compact open subsets on $\mathbb{B}$ converging to $\mathbb{B}$. Also, as

$$
e_{n}^{g}:=L_{g}^{\star}\left(e_{n}\right)=\int_{\mathbb{B}} \psi\left(g^{\prime}\right) R_{g^{\prime}}^{\star}\left(\chi_{C_{n}^{g}}\right) \mathrm{d}_{\mathbb{B}}\left(g^{\prime}\right) \in \mathcal{D}(\mathbb{B}),
$$

we deduce that for all $j, k \in \mathbb{N}$ :

$$
\left\|L_{g}^{*}\left(F_{n}\right)-L_{g}^{*}(F)\right\|_{j, k, \underline{\gamma}}=\left\|\left(1-e_{n}^{g}\right) L_{g}^{*}(F)\right\|_{j, k, \underline{\gamma}} \quad \text { with } \quad \underline{\hat{\gamma}}=\left\{\hat{\mu}_{j}^{R_{j}}\right\}_{j \in \mathbb{N}}
$$

which, by Lemma 1.12 (vi), converges to zero as $L_{g}^{*}(F) \in \mathcal{B} \underline{\underline{\gamma}}(\mathbb{B}, \mathcal{A})$ with $\underline{\gamma}=\left\{\mu_{j}^{R_{j}}\right\}$ and $\underline{\gamma} \prec \underline{\hat{\gamma}}$.

Let $\mathcal{S}^{S_{\text {can }}^{\mathbb{B}}}(\mathbb{B}, \mathcal{A})$ be the one-variable Schwartz space associated to the admissible and tame tempered pair $\left(\mathbb{B} \times \mathbb{B}, S_{\text {can }}^{\mathbb{B}}\right)$, constructed in Definition 1.48. The next result follows immediately from Proposition 1.50 ,

Proposition 3.10. Let $\mathbb{B}$ be a normal $\mathbf{j}$-group and $\vec{\tau} \in \mathbf{\Theta}^{N}$. Then, endowed with the multiplication $\star_{\theta, \vec{\tau}}$, the space $\mathcal{S}^{S_{\mathrm{can}}^{\mathrm{B}}}(\mathbb{B}, \mathcal{A})$ becomes a Fréchet algebra which, for $\underline{\mu}$ an arbitrary family of tempered weights, acts continuously on $\mathcal{B} \underline{\mu}(\mathbb{B}, \mathcal{A})$, via

$$
L_{\star_{\theta, \vec{\tau}}}(F): \varphi \mapsto F \star_{\theta, \vec{\tau}} \varphi, \quad F \in \mathcal{B}^{\mu}(\mathbb{B}, \mathcal{A}), \quad \varphi \in \mathcal{S}^{S_{\text {can }}^{\mathbb{B}}(\mathbb{B}, \mathcal{A}) .}
$$

In particular, $\left(\mathcal{S}^{S_{\text {can }}^{\mathbb{B}}}(\mathbb{B}, \mathcal{A}), \star_{\theta, \vec{\tau}}\right)$ is an ideal of $\left(\mathcal{B}(\mathbb{B}, \mathcal{A}), \star_{\theta, \vec{\tau}}\right)$.

We now see that, as expected, the constant function is an identity for the deformed product.

Proposition 3.11. Let $\mathbb{B}$ be a normal $\mathbf{j}$-group. Fix $\vec{\tau} \in \mathbf{\Theta}^{N}, \underline{\mu}$ a family of tempered weights of sub-multiplicative degree $(\underline{L}, \underline{R})$ and $F \in \mathcal{B} \underline{\mu}(\mathbb{B}, \mathcal{A})$. Identifying an element $a \in \mathcal{A}$ with the function $[g \mapsto a]$ in $\mathcal{B}(\mathbb{B}, \mathcal{A})$, we have

$$
a \star_{\theta, \vec{\tau}} F=a F, \quad F \star_{\theta, \vec{\tau}} a=F a,
$$

in $\mathcal{B}=\underline{\mu}(\mathbb{B}, \mathcal{A})$. In particular, if $\mathcal{A}$ is unital, the element $\left[g \mapsto 1_{\mathcal{A}}\right] \in \mathcal{B}(\mathbb{B}, \mathcal{A})$ is the unit of $\left(\mathcal{B}(\mathbb{B}, \mathcal{A}), \star_{\theta, \vec{\tau}}\right)$.

Proof. Since the constant unit function is a fixed point of the map $T_{\theta, \vec{\tau}}^{-1}$, for every $\varphi \in \mathcal{S}^{S_{\text {can }}^{\mathbb{B}}}(\mathbb{B}, \mathcal{A})$, we have:

$$
\varphi \star_{\theta, \vec{\tau}} a=T_{\theta, \vec{\tau}}\left(T_{\theta, \vec{\tau}}^{-1}(\varphi) \star_{\theta}^{0} a\right),
$$

in $\mathcal{S}_{\text {can }}^{\mathbb{B}}(\mathbb{B}, \mathcal{A})$. By Remark 2.36, we see that the transported Schwartz space $\mathcal{S}^{S_{\text {can }}^{\mathbb{B}}}(\mathbb{B}, \mathcal{A})$ is a (dense) subset of the ordinary Schwartz space $\mathcal{S}(\mathfrak{b}, \mathcal{A})$, under the usual identification $\mathbb{B} \simeq \mathfrak{b}$. Since $T_{\theta, \vec{\tau}}^{-1}$ preserves the latter space, we see that $T_{\theta, \vec{\tau}}^{-1}(\varphi) \in \mathcal{S}(\mathfrak{b}, \mathcal{A})$. It is well known (see [26 for the Fréchet algebra valued case) that the Moyal product admits the constant function as unit element. Thus $\varphi \star_{\theta, \vec{\tau}} a=\varphi a$ and $a \star_{\theta, \vec{\tau}} \varphi=a \varphi$ for all $\varphi \in \mathcal{S}^{S_{\text {can }}^{\mathbb{B}}}(\mathbb{B}, \mathcal{A})$ and $a \in \mathcal{A}$. Now, consider the injective homomorphism $L_{\star_{\theta, \vec{\tau}}}$ from $\left(\mathcal{B}^{\mu}(\mathbb{B}, \mathcal{A}), \star_{\theta, \vec{\tau}}\right)$ to the algebra of continuous operators acting on $\mathcal{S}^{S_{\text {can }}^{\mathbb{B}}}(\mathbb{B}, \mathcal{A})$, defined in Proposition 3.10 From the previous considerations, the associativity of the deformed product and the fact that $\mathcal{S}^{S_{\text {can }}^{\mathbb{B}}}(\mathbb{B}, \mathcal{A})$ is an ideal of $\mathcal{B} \underline{\mu}(\mathbb{B}, \mathcal{A})$, we get

$$
L_{\star_{\theta, \vec{\tau}}}\left(F \star_{\theta, \vec{\tau}} a\right)=L_{\star_{\theta, \vec{\tau}}}(F a), \quad \forall F \in \mathcal{B}^{\mu}(\mathbb{B}, \mathcal{A}),
$$


which entails by injectivity that $F \star_{\theta, \vec{\tau}} a=F a$ in $\mathcal{B} \underline{\nu}(\mathbb{B}, \mathcal{A})$, with $\underline{\nu}=\left\{\mu_{j}^{L_{j}}\right\}$. As $F a \in \mathcal{B}=(\mathbb{B}, \mathcal{A})$, we deduce that the equality $F \star_{\theta, \vec{\tau}} a=F a$ holds in fact in $\mathcal{B}=\underline{\mu}(\mathbb{B}, \mathcal{A})$. The case of $a \star_{\theta, \vec{\tau}} F$ is entirely similar. 


\section{CHAPTER 4}

\section{Deformation of Fréchet algebras}

In this chapter, we consider a normal $\mathbf{j}$-group $\mathbb{B}$ and a pair $(\mathcal{A}, \alpha)$, consisting of a Fréchet algebra $\mathcal{A}$, together with a strongly continuous (not necessarily isometric) action $\alpha$ of $\mathbb{B}$ by automorphisms. For $a \in \mathcal{A}$, we let $\alpha(a)$ be the $\mathcal{A}$-valued function on $\mathbb{B}$, defined by

$$
\alpha(a):=\left[g \in \mathbb{B} \mapsto \alpha_{g}(a) \in \mathcal{A}\right] .
$$

Our main goal, achieved in section 4.1, is to show that the formula

$$
a \star_{\theta, \vec{\tau}}^{\alpha} b:=\left(\alpha(a) \star_{\theta, \vec{\tau}} \alpha(b)\right)(e),
$$

equips $\mathcal{A}^{\infty}$ with a new noncommutative and associative Fréchet algebra structure. This is proved in Theorem 4.8 as a direct consequence of Theorem 3.9 and of Lemma 4.5 which shows that the map (4.1) is continuous from $\mathcal{A}^{\infty}$ to $\mathcal{B} \underline{\mu}\left(\mathbb{B}, \mathcal{A}^{\infty}\right)$, for $\underline{\mu}$ a nontrivial family of tempered weights on the group $\mathbb{B}$.

\subsection{The deformed product}

We do not yet need to work with isometric actions. We start by general considerations regarding tempered actions:

Definition 4.1. A tempered action of a tempered Lie group $G$ on a Fréchet algebra $\mathcal{A}$, is given by the data $\left(\alpha, \underline{\mu}^{\alpha}\right)$ where $\alpha$ is an action of $G$ on $\mathcal{A}$ and $\underline{\mu}^{\alpha}$ is a family of tempered weights on $G$ such that for all $j \in \mathbb{N}$, all $a \in \mathcal{A}$ and all $\bar{g} \in G$, we have

$$
\left\|\alpha_{g}(a)\right\|_{j} \leq \mu_{j}^{\alpha}(g)\|a\|_{j} .
$$

REMARK 4.2. Note that for a tempered action and for $g \in G$ fixed, $\alpha_{g}$ acts continuously on $\mathcal{A}$.

We denote by $\mathcal{A}^{\infty}$ the set of smooth vectors for the action $\alpha$ of $\mathbb{B}$ on $\mathcal{A}$ :

$$
\mathcal{A}^{\infty}:=\left\{a \in \mathcal{A}: \alpha(a) \in C^{\infty}(\mathbb{B}, \mathcal{A})\right\} .
$$

When the action is strongly continuous, $\mathcal{A}^{\infty}$ is a dense subspace of $\mathcal{A}$. On this subset, we consider the infinitesimal form of the action, given for $X \in \mathfrak{b}$ by:

$$
X^{\alpha}(a):=\left.\frac{d}{d t}\right|_{t=0} \alpha_{e^{t X}}(a), \quad a \in \mathcal{A}^{\infty},
$$

and extended to the whole universal enveloping algebra $\mathcal{U}(\mathfrak{b})$, by declaring that the map $\mathcal{U}(\mathfrak{b}) \rightarrow \operatorname{End}\left(\mathcal{A}^{\infty}\right), X \mapsto X^{\alpha}$ is an algebra homomorphism. The subspace $\mathcal{A}^{\infty}$ carries a finer topology associated with the following set of semi-norms:

$$
\|a\|_{j, X}:=\left\|X^{\alpha}(a)\right\|_{j}, \quad a \in \mathcal{A}^{\infty}, \quad X \in \mathcal{U}(\mathfrak{b}), j \in \mathbb{N} .
$$

Considering the PBW basis of $\mathcal{U}(\mathfrak{b})$ associated to an ordered basis of $\mathfrak{b}$ as in (0.4), one can use only countably many semi-norms to define the topology of $\mathcal{A}^{\infty}$. The 
latter are indexed by $(j, k) \in \mathbb{N}^{2}$, where $j$ refers to the labeling of the initial family of semi-norms $\left\{\|\cdot\|_{j}\right\}_{j \in \mathbb{N}}$ of $\mathcal{A}$ and $k$ refers to the labeling of the filtration $\mathcal{U}(\mathfrak{b})=$ $\cup_{k \in \mathbb{N}} \mathcal{U}_{k}(\mathfrak{b})$ associated to the chosen PBW basis, as defined in (0.5). In turn, $\mathcal{A}^{\infty}$ becomes a Fréchet space, for the topology associated with the semi-norms

$$
\|\cdot\|_{j, k}: \mathcal{A}^{\infty} \rightarrow[0, \infty), \quad a \mapsto \sup _{X \in \mathcal{U}_{k}(\mathfrak{g})} \frac{\|a\|_{j, X}}{|X|_{k}}=\sup _{X \in \mathcal{U}_{k}(\mathfrak{g})} \frac{\left\|X^{\alpha}(a)\right\|_{j}}{|X|_{k}}
$$

with $j, k \in \mathbb{N}$ and where $|.|_{k}$ is the $\ell^{1}$-norm of $\mathcal{U}_{k}(\mathfrak{b})$ defined in (0.7). As in (1.4), we have

$$
\|a\|_{j, k} \leq \max _{|\beta| \leq k}\|a\|_{j, X^{\beta}},
$$

with $\left\{X^{\beta},|\beta| \leq k\right\}$ the basis (0.4) of $\mathcal{U}_{k}(\mathfrak{b})$. Hence the semi-norms (4.2) are well defined on $\mathcal{A}^{\infty}$.

In the context of a tempered action on a Fréchet algebra $\mathcal{A}$, we observe that the restriction of the action to $\mathcal{A}^{\infty}$ is also tempered, but never isometric, even if the action is isometric on $\mathcal{A}$ unless the group is Abelian. This explains why in our context it is natural to work with tempered actions, rather than with isometric ones.

Lemma 4.3. Let $\left(\mathcal{A}, \alpha, \mu^{\alpha}\right)$ be a Fréchet algebra endowed with a tempered action of a tempered Lie group $G$. Then, the restriction of $\alpha$ on $\mathcal{A}^{\infty}$ is tempered too, with:

$$
\left\|\alpha_{g}(a)\right\|_{j, k} \leq C(k) \mathfrak{d}_{G}(g)^{k} \mu_{j}^{\alpha}(g)\|a\|_{j, k}, \quad j, k \in \mathbb{N}, g \in G, a \in \mathcal{A}^{\infty} .
$$

Proof. First remark

$$
\left\|\alpha_{g}(a)\right\|_{j, k}=\sup _{X \in \mathcal{U}_{k}(\mathfrak{g})} \frac{\left\|\alpha_{g}\left(\left(\operatorname{Ad}_{g^{-1}}(X)\right)^{\alpha}(a)\right)\right\|_{j}}{|X|_{k}} \leq \mu_{j}^{\alpha}(g) \sup _{X \in \mathcal{U}_{k}(\mathfrak{g})} \frac{\left\|\left(\operatorname{Ad}_{g^{-1}}(X)\right)^{\alpha}(a)\right\|_{j}}{|X|_{k}} .
$$

As for $X \in \mathcal{U}_{k}(\mathfrak{g})$ and $a \in \mathcal{A}^{\infty}$, we have

$$
\left\|X^{\alpha}(a)\right\|_{j} \leq|X|_{k} \sup _{Y \in \mathcal{U}_{k}(\mathfrak{g})} \frac{\left\|Y^{\alpha}(a)\right\|_{j}}{|Y|_{k}}=|X|_{k}\|a\|_{j, k},
$$

we get, with $\left|\operatorname{Ad}_{g}\right|_{k}$ denoting the operator norm of the adjoint action of $G$ on the normed space $\left(\mathcal{U}_{k}(\mathfrak{g}),|\cdot|_{k}\right)$ :

$$
\left\|\alpha_{g}(a)\right\|_{j, k} \leq \mu_{j}^{\alpha}(g) \sup _{X \in \mathcal{U}_{k}(\mathfrak{g})} \frac{\left|\operatorname{Ad}_{g^{-1}}(X)\right|_{k}}{|X|_{k}}\|a\|_{j, k}=\mu_{j}^{\alpha}(g)\left|\operatorname{Ad}_{g^{-1}}\right|_{k}\|a\|_{j, k},
$$

and one concludes using Lemma 1.14

EXAMPLE 4.4. Applying the former result to $\alpha=R^{\star}$ and $\mathcal{A}=\mathcal{S}^{S_{\text {can }}^{\mathbb{B}}}(\mathbb{B})$ (which is its own space of smooth vectors), we see that the right-action of $\mathbb{B}$ on $\mathcal{S}^{S_{\text {can }}^{\mathbb{B}}}(\mathbb{B})$ is tempered.

The following statement is the foundation of our construction:

Lemma 4.5. Let $\left(\alpha, \underline{\mu}^{\alpha}\right)$ be a tempered and strongly continuous action of a Lie group $G$ on a Fréchet algebra $\mathcal{A}$. Setting then $\underline{\nu}:=\left\{\mu_{j}^{\alpha} \mathfrak{d}_{G}^{k}\right\}_{j, k \in \mathbb{N}}$, we have an equivariant continuous embedding

$$
\alpha: \mathcal{A}^{\infty} \rightarrow \mathcal{B}^{\underline{\nu}}\left(G, \mathcal{A}^{\infty}\right), \quad a \mapsto \alpha(a)=\left[g \in G \mapsto \alpha_{g}(a) \in \mathcal{A}^{\infty}\right] .
$$


Proof. Note first that for $a \in \mathcal{A}$ and $g, g_{0} \in G$, we have

$$
\alpha\left(\alpha_{g}(a)\right)\left(g_{0}\right)=\alpha_{g_{0} g}(a)=\left(R_{g}^{\star} \alpha(a)\right)\left(g_{0}\right),
$$

and thus $\alpha: a \in \mathcal{A} \mapsto\left[g \mapsto \alpha_{g}(a)\right] \in C(G, \mathcal{A})$ intertwines the actions $R^{\star}$ and $\alpha$. Let now $a \in \mathcal{A}^{\infty}$ and $X \in \mathcal{U}(\mathfrak{g})$. By equivariance and strong-differentiability of $\alpha$ on $\mathcal{A}^{\infty}$, we get

$$
\widetilde{X} \alpha(a)=\alpha\left(X^{\alpha} a\right) .
$$

Since for all $j \in \mathbb{N}$ and all $a \in \mathcal{A}$, we have $\left\|\alpha_{g}(a)\right\|_{j} \leq \mu_{j}^{\alpha}(g)\|a\|_{j}$, we deduce that

$$
\begin{aligned}
\|\alpha(a)\|_{j, k, \underline{\mu}^{\alpha}}=\sup _{X \in \mathcal{U}_{k}(\mathfrak{g})} \sup _{g \in G} \frac{\left\|\tilde{X} \alpha_{g}(a)\right\|_{j}}{\mu_{j}^{\alpha}(g)|X|_{k}} & =\sup _{X \in \mathcal{U}_{k}(\mathfrak{g})} \sup _{g \in G} \frac{\left\|\alpha_{g}\left(X^{\alpha} a\right)\right\|_{j}}{\mu_{j}^{\alpha}(g)|X|_{k}} \\
& \leq \sup _{X \in \mathcal{U}_{k}(\mathfrak{g})} \frac{\left\|X^{\alpha} a\right\|_{j}}{|X|_{k}}=\|a\|_{j, k} .
\end{aligned}
$$

This analysis shows that the map $\alpha: \mathcal{A}^{\infty} \rightarrow \mathcal{B}^{\mu^{\alpha}}(G, \mathcal{A})$ is continuous. Now we want to take into account the intrinsic topology of $\mathcal{A}^{\infty}$ in the target space of the map $\alpha$. Remark that the topology of $\mathcal{B} \underline{\nu}\left(G, \mathcal{A}^{\infty}\right)$ is associated with the countable set of semi-norms

$$
\|F\|_{(j, k), k^{\prime}, \underline{\nu}}=\sup _{X \in \mathcal{U}_{k^{\prime}}(\mathfrak{g})} \sup _{g \in G} \sup _{Y \in \mathcal{U}_{k}(\mathfrak{g})} \frac{\left\|Y^{\alpha}(\widetilde{X} F(g))\right\|_{j}}{\nu_{j, k}(g)|X|_{k^{\prime}}|Y|_{k}} .
$$

Since $\alpha_{g^{-1}} \circ X^{\alpha} \circ \alpha_{g}=\left(\operatorname{Ad}_{g^{-1}} X\right)^{\alpha}$ for all $X \in \mathcal{U}(\mathfrak{g})$ and $g \in G$, we get for $F=\alpha(a)$ and $\underline{\nu}=\left\{\mu_{j}^{\alpha} \mathfrak{d}_{G}^{k}\right\}_{j, k \in \mathbb{N}}$ :

$$
\begin{aligned}
\|\alpha(a)\|_{(j, k), k^{\prime}, \underline{\nu}} & =\sup _{X \in \mathcal{U}_{k^{\prime}}(\mathfrak{g})} \sup _{g \in G} \sup _{Y \in \mathcal{U}_{k}(\mathfrak{g})} \frac{\left\|Y^{\alpha}\left(\widetilde{X}_{g} \alpha_{g}(a)\right)\right\|_{j}}{\mu_{j}^{\alpha}(g) \mathfrak{d}_{G}(g)^{k}|X|_{k^{\prime}}|Y|_{k}} \\
& =\sup _{X \in \mathcal{U}_{k^{\prime}}(\mathfrak{g})} \sup _{g \in G} \sup _{Y \in \mathcal{U}_{k}(\mathfrak{g})} \frac{\left\|Y^{\alpha}\left(\alpha_{g}\left(X^{\alpha} a\right)\right)\right\|_{j}}{\mu_{j}^{\alpha}(g) \mathfrak{d}_{G}(g)^{k}|X|_{k^{\prime}}|Y|_{k}} \\
& =\sup _{X \in \mathcal{U}_{k^{\prime}}(\mathfrak{g})} \sup _{g \in G} \sup _{Y \in \mathcal{U}_{k}(\mathfrak{g})} \frac{\left\|\alpha_{g}\left(\left(\operatorname{Ad}_{g^{-1}} Y\right)^{\alpha} X^{\alpha} a\right)\right\|_{j}}{\mu_{j}^{\alpha}(g) \mathfrak{d}_{G}(g)^{k}|X|_{k^{\prime}}|Y|_{k}} \\
& \leq \sup _{X \in \mathcal{U}_{k^{\prime}}(\mathfrak{g})} \sup _{g \in G} \sup _{Y \in \mathcal{U}_{k}(\mathfrak{g})} \frac{\left\|\left(\operatorname{Ad}_{g^{-1}} Y\right)^{\alpha} X^{\alpha} a\right\|_{j}}{\left.\mathfrak{d}_{G}(g)^{k}|X|\right|_{k^{\prime}}|Y|_{k}} \\
& \leq\left(\sup _{g \in G} \frac{\left|\operatorname{Ad}_{g}\right|_{k}}{\mathfrak{d}_{G}(g)^{k}} \sup _{X \in \mathcal{U}_{k^{\prime}}(\mathfrak{g})} \sup _{Y \in \mathcal{U}_{k}(\mathfrak{g})} \frac{\left\|Y^{\alpha} X^{\alpha} a\right\|_{j}}{|X|_{k^{\prime}}|Y|_{k}}\right. \\
& \leq\left(\sup _{g \in G} \frac{\left|\operatorname{Ad}_{g^{-1}}\right|_{k}}{\mathfrak{d}_{G}(g)^{k}}\right)\left(\sup _{X \in \mathcal{U}_{k^{\prime}}(\mathfrak{g})} \sup _{Y \in \mathcal{U}_{k}(\mathfrak{g})} \frac{|Y X|_{k+k^{\prime}}}{|X|_{k^{\prime}}|Y|_{k}}\right)_{Z \in \mathcal{U}_{k+k^{\prime}}(\mathfrak{g})} \frac{\left\|Z^{\alpha} a\right\|_{j}}{|Z|_{k+k^{\prime}}} \\
& =\left(\sup _{g \in G} \frac{\left|\operatorname{Ad}_{g^{-1}}\right|_{k}}{\mathfrak{d}_{G}(g)^{k}}\right)\left(\sup _{X \in \mathcal{U}_{k^{\prime}}(\mathfrak{g})} \sup _{Y \in \mathcal{U}_{k}(\mathfrak{g})} \frac{|Y X|_{k+k^{\prime}}}{|X|_{k^{\prime}}|Y|_{k}}\right)\|a\|_{j, k+k^{\prime}},
\end{aligned}
$$

and one concludes using Lemma 1.14

The next result, although rather obvious, will also play a key role.

Lemma 4.6. Let $\mathcal{A}$ be a Fréchet algebra and let $\underline{\mu}$ be a family of tempered weights on a tempered Lie group $G$. Then, the evaluation map at the unit element, $\mathcal{B}^{\mu}(G, \mathcal{A}) \rightarrow \mathcal{A}, F \mapsto F(e)$, is continuous. 
Proof. Fix $j \in \mathbb{N}$. We have for any $F \in \mathcal{B}^{\underline{\mu}}(G, \mathcal{A})$ :

$$
\|F(e)\|_{j} \leq \mu_{j}(e) \sup _{g \in G} \frac{\|F(g)\|_{j}}{\mu_{j}(g)}=\mu_{j}(e)\|F\|_{j, 0, \underline{\mu}},
$$

and the result follows immediately.

Last, we need to lift the action $\alpha$ from $\mathcal{A}^{\infty}$ to $\mathcal{B} \underline{\nu}\left(\mathbb{B}, \mathcal{A}^{\infty}\right), \underline{\nu}=\left\{\mu_{j}^{\alpha} \mathfrak{d}_{G}^{k}\right\}$, and to show that this lift acts by automorphisms of the product $\star_{\theta, \vec{\tau}}$.

LEMma 4.7. Let $\left(\alpha, \underline{\mu}^{\alpha}\right)$ be a strongly continuous and tempered action of a normal $\mathbf{j}$-group $\mathbb{B}$ on a Fréchet algebra $\mathcal{A}$ and $\underline{\mu}_{1}, \underline{\mu}_{2}$ be two families of tempered weights with sub-multiplicative degree $\left(\underline{L}_{1}, \underline{R}_{1}\right),\left(\underline{L}_{2}, \underline{R}_{2}\right)$. For $g \in \mathbb{B}$, the map

$$
\hat{\alpha}_{g}: F \mapsto\left[g_{0} \in \mathbb{B} \mapsto \alpha_{g}\left(F\left(g_{0}\right)\right)\right],
$$

is continuous on $\mathcal{B}^{\mu}{ }_{i}\left(\mathbb{B}, \mathcal{A}^{\infty}\right), i=1,2$. Moreover, given $(\theta, \vec{\tau}) \in \mathbb{R}^{*} \times \boldsymbol{\Theta}^{N}$, $\hat{\alpha}$ defines an action of $\mathbb{B}$ by automorphisms of the deformed product $\star_{\theta, \vec{\tau}}$, in the sense that for all $F_{1} \in \mathcal{B}^{\underline{\mu}}\left(\mathbb{B}, \mathcal{A}^{\infty}\right)$ and $F_{1} \in \mathcal{B}^{\underline{\mu}}-\left(\mathbb{B}, \mathcal{A}^{\infty}\right)$, we have for all $g \in \mathbb{B}$ :

$$
\hat{\alpha}_{g}\left(F \star_{\theta, \vec{\tau}} F^{\prime}\right)=\hat{\alpha}_{g}(F) \star_{\theta, \vec{\tau}} \hat{\alpha}_{g}\left(F^{\prime}\right) \quad \text { in } \quad \mathcal{B}^{\underline{\nu}}\left(\mathbb{B}, \mathcal{A}^{\infty}\right),
$$

with

$$
\underline{\nu}=\left\{\mu_{1, j, k}^{L_{1, j, k}} \mu_{2, j, k}^{L_{2, j, k}}\right\}_{j, k \in \mathbb{N}} .
$$

Proof. For $F \in \mathcal{B}^{\mu}\left(\mathbb{B}, \mathcal{A}^{\infty}\right), X, Y \in \mathcal{U}(\mathfrak{b})$ and $g, g^{\prime} \in \mathbb{B}$, we have

$$
Y^{\alpha}\left(\widetilde{X} \hat{\alpha}_{g}(F)\left(g^{\prime}\right)\right)=\alpha_{g}\left(\left(\operatorname{Ad}_{g^{-1}} Y\right)^{\alpha}\left(\widetilde{X} F\left(g^{\prime}\right)\right)\right) .
$$

This entails that

$$
\begin{aligned}
&\left\|\hat{\alpha}_{g}(F)\right\|_{(j, k), k^{\prime}, \underline{\mu}}= \sup _{X \in \mathcal{U}_{k^{\prime}}(\mathfrak{b})} \sup _{g^{\prime} \in \mathbb{B}} \sup _{Y \in \mathcal{U}_{k}(\mathfrak{b})} \frac{\left\|Y^{\alpha}\left(\widetilde{X} \hat{\alpha}_{g}(F)\left(g^{\prime}\right)\right)\right\|_{j}}{\mu_{j, k}\left(g^{\prime}\right)|X|_{k^{\prime}}|Y|_{k}} \\
&= \sup _{X \in \mathcal{U}_{k^{\prime}}(\mathfrak{b})} \sup _{g^{\prime} \in \mathbb{B}} \sup _{Y \in \mathcal{U}_{k}(\mathfrak{b})} \frac{\left\|\alpha_{g}\left(\left(\operatorname{Ad}_{g^{-1}} Y\right)^{\alpha}\left(\widetilde{X} F\left(g^{\prime}\right)\right)\right)\right\|_{j}}{\mu_{j, k}\left(g^{\prime}\right)|X|_{k^{\prime}}|Y|_{k}} \\
& \leq C(k) \mu_{j}^{\alpha}(g) \mathfrak{d}_{\mathbb{B}}(g)^{k} \sup _{X \in \mathcal{U}_{k^{\prime}}(\mathfrak{b})} \sup _{g^{\prime} \in \mathbb{B}} \sup _{Y \in \mathcal{U}_{k}(\mathfrak{b})} \frac{\left\|Y^{\alpha}\left(\widetilde{X} F\left(g^{\prime}\right)\right)\right\|_{j}}{\mu_{j, k}\left(g^{\prime}\right)|X|_{k^{\prime}}|Y|_{k}} \\
& \quad=C(k) \mu_{j}^{\alpha}(g) \mathfrak{d}_{\mathbb{B}}(g)^{k}\|F\|_{(j, k), k^{\prime}, \underline{\mu}},
\end{aligned}
$$

proving the continuity.

Next, consider $F_{1} \in \mathcal{B}^{\underline{\mu}} \underline{ }_{1}\left(\mathbb{B}, \mathcal{A}^{\infty}\right)$ and $F_{2} \in \mathcal{B}^{\underline{\mu}}{ }_{2}\left(\mathbb{B}, \mathcal{A}^{\infty}\right)$, together with $\underline{\hat{\mu}}_{1}$ and $\hat{\mu}_{2}$, two families of tempered weights that dominate respectively $\underline{\mu}_{1}$ and $\underline{\mu}_{2}$. Defining $\bar{F}_{1, n}:=F_{1} e_{n} \in \mathcal{D}(\mathbb{B}, \mathcal{A})$ and $F_{2, n}=F_{2} e_{n} \in \mathcal{D}(\mathbb{B}, \mathcal{A})$, with $e_{n} \in \mathcal{D}(\bar{B})$ defined in (1.7), from

$$
\hat{\alpha}_{g}\left(F_{1, n}\right)=\hat{\alpha}_{g}\left(F_{1}\right) e_{n}, \quad \hat{\alpha}_{g}\left(F_{2, n}\right)=\hat{\alpha}_{g}\left(F_{2}\right) e_{n},
$$

we deduce from Lemma 1.12 (viii) that $\left\{\hat{\alpha}_{g}\left(F_{1, n}\right)\right\}$ and $\left\{\hat{\alpha}_{g}\left(F_{2, n}\right)\right\}$ converges to $\left\{\hat{\alpha}_{g}\left(F_{1}\right)\right\}$ and $\left\{\hat{\alpha}_{g}\left(F_{2}\right)\right\}$ in the topologies of $\mathcal{B}^{\hat{\mu}_{1}}\left(\mathbb{B}, \mathcal{A}^{\infty}\right)$ and $\mathcal{B} \underline{\hat{\mu}}_{2}\left(\mathbb{B}, \mathcal{A}^{\infty}\right)$ respectively. Thus, we can use Lemma 1.44 to get the $\hat{\alpha}$-equivariance at the level of smooth compactly supported functions from the commutativity of $\hat{\alpha}$ and $R^{\star}$ :

$$
\begin{aligned}
\hat{\alpha}_{g}\left(F \star_{\theta, \vec{\tau}} F^{\prime}\right) & =\hat{\alpha}_{g}\left(\lim _{n, n^{\prime} \rightarrow \infty} F_{n} \star_{\theta, \vec{\tau}} F_{n^{\prime}}^{\prime}\right)=\lim _{n, n^{\prime} \rightarrow \infty} \hat{\alpha}_{g}\left(F_{n} \star_{\theta, \vec{\tau}} F_{n^{\prime}}^{\prime}\right) \\
& =\lim _{n, n^{\prime} \rightarrow \infty} \hat{\alpha}_{g}\left(F_{n}\right) \star_{\theta, \vec{\tau}} \hat{\alpha}_{g}\left(F_{n^{\prime}}^{\prime}\right)=\hat{\alpha}_{g}(F) \star_{\theta, \vec{\tau}} \hat{\alpha}_{g}\left(F^{\prime}\right),
\end{aligned}
$$


in $\mathcal{B} \underline{\nu}\left(\mathbb{B}, \mathcal{A}^{\infty}\right)$, with $\underline{\nu}=\left\{\mu_{1, j, k}^{L_{1, j, k}} \mu_{2, j, k} L_{2, j, k}\right\}$.

We are now prepared to state the main result of the first part of this memoir:

Theorem 4.8 (Universal Deformation Formula of Fréchet Algebras). Let $(\mathcal{A}, \alpha)$ be a Fréchet algebra endowed with a tempered and strongly continuous action of a normal $\mathbf{j}$-group $\mathbb{B}$. Let also $\theta \in \mathbb{R}^{*}$ and $\vec{\tau} \in \Theta^{N}$. Then, $\left(\mathcal{A}^{\infty}, \star_{\theta, \vec{\tau}}^{\alpha}\right)$ is an associative Fréchet algebra with continuous product.

Proof. Let $\underline{\mu}^{\alpha}$ be the family of tempered weights, with sub-multiplicative degree $(\underline{L}, \underline{R})$, associated with the tempered action $\alpha$ as in Definition 4.1 Let $a, b \in \mathcal{A}^{\infty}$, then by Lemma 4.5 $\alpha(a), \alpha(b) \in \mathcal{B} \underline{\mu}\left(\mathbb{B}, \mathcal{A}^{\infty}\right)$, where $\underline{\mu}=\left\{\mu_{j}^{\alpha} \mathfrak{d}_{\mathbb{B}}^{k}\right\}$. Then, since $\mathfrak{d}_{\mathbb{B}}$ is sub-multiplicative of degree $(1,1)$, Theorem 3.9 shows that $\alpha(a) \star_{\theta, \vec{\tau}} \alpha(b)$ belongs to $\mathcal{B} \underline{\underline{ }}\left(\mathbb{B}, \mathcal{A}^{\infty}\right)$, for $\underline{\nu}=\left\{\mu_{j}^{\alpha 2 L_{j}} \mathfrak{d}_{\mathbb{B}}^{2 k}\right\}$, and that the map

$$
\mathcal{A}^{\infty} \times \mathcal{A}^{\infty} \rightarrow \mathcal{B} \underline{\underline{\nu}}\left(\mathbb{B}, \mathcal{A}^{\infty}\right), \quad(a, b) \mapsto \alpha(a) \star_{\theta, \vec{\tau}} \alpha(b),
$$

is continuous. Applying Lemma 4.6 for the Fréchet algebra $\mathcal{A}^{\infty}$ then yields that the composition of maps

$$
\begin{aligned}
& \mathcal{A}^{\infty} \times \mathcal{A}^{\infty} \rightarrow \mathcal{B} \underline{\nu}\left(\mathbb{B}, \mathcal{A}^{\infty}\right) \rightarrow \mathcal{A}^{\infty}, \\
& (a, b) \mapsto \alpha(a) \star_{\theta, \vec{\tau}} \alpha(b) \mapsto\left(\alpha(a) \star_{\theta, \vec{\tau}} \alpha(b)\right)(e)=: a \star_{\theta, \vec{\tau}}^{\alpha} b,
\end{aligned}
$$

is continuous.

It remains to prove associativity. With $\hat{\alpha}$ defined in Lemma 4.7, we compute for $a, b \in \mathcal{A}^{\infty}$ and $g \in \mathbb{B}$ :

$$
\alpha\left(a \star_{\theta, \vec{\tau}}^{\alpha} b\right)(g)=\alpha_{g}\left(a \star_{\theta, \vec{\tau}}^{\alpha} b\right)=\alpha_{g}\left(\alpha(a) \star_{\theta, \vec{\tau}} \alpha(b)(e)\right)=\hat{\alpha}_{g}\left(\alpha(a) \star_{\theta, \vec{\tau}} \alpha(b)\right)(e) .
$$

Using Lemma 4.7 we deduce the equality in $\mathcal{B} \underline{\underline{\nu}}\left(\mathbb{B}, \mathcal{A}^{\infty}\right.$ ) (for the value of $\underline{\nu}$ as indicated above):

$$
\hat{\alpha}_{g}\left(\alpha(a) \star_{\theta, \vec{\tau}} \alpha(b)\right)=\hat{\alpha}_{g}(\alpha(a)) \star_{\theta, \vec{\tau}} \hat{\alpha}_{g}(\alpha(b)) .
$$

As a short computation shows, for $a \in \mathcal{A}$ and $g \in \mathbb{B}$, we have $\hat{\alpha}_{g}(\alpha(a))=$ $L_{g^{-1}}^{\star}(\alpha(a))$. Thus, using the equivariance of the product $\star_{\theta, \vec{\tau}}$ under the left regular action, as stated in Theorem 3.9] we get the equalities

$$
\hat{\alpha}_{g}(\alpha(a)) \star_{\theta, \vec{\tau}} \hat{\alpha}_{g}(\alpha(b))=L_{g^{-1}}^{\star}(\alpha(a)) \star_{\theta, \vec{\tau}} L_{g^{-1}}^{\star}(\alpha(b))=L_{g^{-1}}^{*}\left(\alpha(a) \star_{\theta, \vec{\tau}} \alpha(b)\right),
$$

in $\mathcal{B} \underline{\lambda}\left(\mathbb{B}, \mathcal{A}^{\infty}\right)$, for $\underline{\lambda}=\left\{\mu_{j}^{\alpha 2 L_{j} R_{j}} \mathfrak{d}_{\mathbb{B}}^{2 k}\right\}$. Evaluating this equality at the unit element, yields, by Lemma 4.6 the equality in $\mathcal{A}^{\infty}$ (remember that $g \in \mathbb{B}$ is fixed):

$$
\alpha\left(a \star_{\theta, \vec{\tau}}^{\alpha} b\right)(g)=L_{g^{-1}}^{*}\left(\alpha(a) \star_{\theta, \vec{\tau}} \alpha(b)\right)(e)=\left(\alpha(a) \star_{\theta, \vec{\tau}} \alpha(b)\right)(g) .
$$

Hence, the functions $\alpha\left(a \star_{\theta, \vec{\tau}}^{\alpha} b\right)$ and $\alpha(a) \star_{\theta, \vec{\tau}} \alpha(b)$ coincide. This implies for $a, b, c \in$ $\mathcal{A}^{\infty}$ :

$$
a \star_{\theta, \vec{\tau}}^{\alpha}\left(b \star_{\theta, \vec{\tau}}^{\alpha} c\right)=\left(\alpha(a) \star_{\theta, \vec{\tau}} \alpha\left(b \star_{\theta, \vec{\tau}}^{\alpha} c\right)\right)(e)=\left(\alpha(a) \star_{\theta, \vec{\tau}}\left(\alpha(b) \star_{\theta, \vec{\tau}} \alpha(c)\right)\right)(e),
$$

and the associativity of $\star_{\theta, \vec{\tau}}^{\alpha}$ on $\mathcal{A}^{\infty}$ follows from associativity of $\star_{\theta, \vec{\tau}}$ on the triple Cartesian product of the space $\mathcal{B} \underline{\nu}(\mathbb{B}, \mathcal{A})$, as stated in Theorem 3.9

Remark 4.9. Contrarily to the $\mathbb{R}^{2 d}$-action case treated in [26, in the nonAbelian situation the original action is no longer an automorphism of the deformed product $\star_{\theta, \vec{\tau}}$ on $\mathcal{A}^{\infty}$. This can be understood as the chief reason to introduce the whole oscillatory integrals machinery in chapter 1 and also to consider the spaces $\mathcal{B}^{\mu}(\mathbb{B}, \mathcal{A})$. 
To conclude this section, we establish a formula for the deformed product $\star_{\theta, \vec{\tau}}^{\alpha}$ on $\mathcal{A}^{\infty}$, which in some sense, is more natural. It will also clarify an important point, namely that the universal deformation of the algebrd $\mathbb{1} \mathcal{A}=C_{r u}(\mathbb{B})$, for the action $\alpha=R^{\star}$ coincides with $\left(\mathcal{B}(\mathbb{B}), \star_{\theta, \vec{\tau}}\right)$.

Proposition 4.10. Let $\left(\alpha, \underline{\mu}^{\alpha}\right)$ be a strongly continuous and tempered action of a normal $\mathbf{j}$-group $\mathbb{B}$ on a Fréchet algebra $\mathcal{A}$. Then, for $a, b \in \mathcal{A}^{\infty}$ and $\theta \in \mathbb{R}^{*}$, $\vec{\tau} \in \mathbf{\Theta}^{N}$, we have

$$
a \star_{\theta, \vec{\tau}}^{\alpha} b=\int_{\mathbb{B} \times \mathbb{B}} K_{\theta, \vec{\tau}}(\alpha(a) \otimes \alpha(b)),
$$

where we denote

$$
\alpha(a) \otimes \alpha(b): \mathbb{B} \times \mathbb{B} \rightarrow \mathcal{A}^{\infty}: \quad(x, y) \mapsto \alpha_{x}(a) \alpha_{y}(b) .
$$

Proof. Since for $a \in \mathcal{A}^{\infty}$, the element $\alpha(a)$ belongs to $\mathcal{B}^{\mu}\left(\mathbb{B}, \mathcal{A}^{\infty}\right), \underline{\mu}=$ $\left\{\mu_{j}^{\alpha} \mathfrak{d}_{\mathbb{B}}^{k}\right\}$, by Lemma 4.5 and the Leibniz rule, we get that

$$
\alpha(a) \otimes \alpha(b) \in \mathcal{B}^{\underline{\mu} \otimes} \underline{\mu}\left(\mathbb{B} \times \mathbb{B}, \mathcal{A}^{\infty}\right),
$$

which shows that the right hand side of (4.3) is indeed well defined. Next, by construction we have

$$
\alpha(a) \star_{\theta, \vec{\tau}} \alpha(b)=\int_{\mathbb{B} \times \mathbb{B}} K_{\theta, \vec{\tau}}(\mathcal{R} \otimes \mathcal{R}(\alpha(a), \alpha(b))) \in \mathcal{B}^{\lambda}\left(\mathbb{B}, \mathcal{A}^{\infty}\right),
$$

with

$$
\underline{\lambda}=\left\{\mu_{j}^{\alpha 2 L_{j} R_{j}} \mathfrak{d}_{\mathbb{B}}^{2 k}\right\},
$$

where the map $\mathcal{R} \otimes \mathcal{R}$ has been defined in Lemma1.42. Now, using Lemma 1.44, we get with the element $e_{n} \in \mathcal{D}(\mathbb{B})$ defined in (1.7), $n \in \mathbb{N}$, the equality in $\mathcal{B} \lambda\left(\mathbb{B}, \mathcal{A}^{\infty}\right)$ :

$$
\alpha(a) \star_{\theta, \vec{\tau}} \alpha(b)=\lim _{n, m \rightarrow \infty} \int_{\mathbb{B} \times \mathbb{B}} K_{\theta, \vec{\tau}}(x, y) R_{x}^{\star}\left(e_{n} \alpha(a)\right) R_{y}^{\star}\left(e_{m} \alpha(b)\right) \mathrm{d}_{\mathbb{B}}(x) \mathrm{d}_{\mathbb{B}}(y) .
$$

By Lemma 4.6, we know that the evaluation at the neutral element is continuous from $\mathcal{B} \lambda\left(\mathbb{B}, \mathcal{A}^{\infty}\right)$ to $\mathcal{A}^{\infty}$. Thus we get

$$
\begin{aligned}
a \star_{\theta, \vec{\tau}}^{\alpha} b & =\left(\alpha(a) \star_{\theta, \vec{\tau}} \alpha(b)\right)(e) \\
& =\lim _{n, m \rightarrow \infty} \int_{\mathbb{B} \times \mathbb{B}} K_{\theta, \vec{\tau}}(x, y) e_{n}(x) \alpha_{x}(a) e_{m}(y) \alpha_{y}(b) \mathrm{d}_{\mathbb{B}}(x) \mathrm{d}_{\mathbb{B}}(y),
\end{aligned}
$$

one then concludes using Proposition 1.32 ,

Corollary 4.11. Let $\theta \in \mathbb{R}^{*}$ and $\vec{\tau} \in \mathbf{\Theta}^{N}$. For $\mathcal{A}=C_{r u}(\mathbb{B})$ and $\alpha=R^{\star}$, we have

$$
\left(\mathcal{A}^{\infty}, \star_{\theta, \vec{\tau}}^{\alpha}\right)=\left(\mathcal{B}(\mathbb{B}), \star_{\theta, \vec{\tau}}\right) .
$$

Proof. By Lemma 1.8 (ii), the set of smooth vectors in $C_{r u}(\mathbb{B})$ for the rightregular action is $\mathcal{B}(\mathbb{B})$. By the Proposition above, their algebraic structures coincide too.

\footnotetext{
${ }^{1}$ Recall that $C_{r u}(\mathbb{B})$ denotes the $C^{*}$-algebra of right uniformly continuous and bounded functions on $\mathbb{B}$, endowed with the sup-norm.
} 


\subsection{Relation with the fixed point algebra}

Under slightly more restrictive conditions on the tempered action $\left(\alpha, \mu^{\alpha}\right)$, we exhibit a relationship between the deformed Fréchet algebra $\left(\mathcal{A}^{\infty}, \star_{\theta, \vec{\tau}}^{\alpha}\right)$ and a fixed point subalgebra of $\left(\mathcal{B}^{\alpha} \underline{\mu}^{\alpha}(\mathbb{B}, \mathcal{A}), \star_{\theta, \vec{\tau}}\right)$. This construction is very similar to the construction of isospectral deformations of Connes and Dubois-Violette [13. So, throughout this paragraph, we still assume that $\mathcal{A}$ is a Fréchet algebra carrying a strongly continuous and tempered action $\alpha$ of a normal $\mathbf{j}$-group $\mathbb{B}$. But now, we further assume that the action is almost-isometric. By this, we mean that there exists a family of tempered weights $\underline{\mu}^{\alpha}$ such that for $a \in \mathcal{A}$ and all $g \in \mathbb{B}$, we have

$$
\left\|\alpha_{g}(a)\right\|_{j}=\mu_{j}^{\alpha}(g)\|a\|_{j} .
$$

ExAmple 4.12. For any Lie group $G$, take $\mathcal{A}=L^{p}(G), p \in[1, \infty)$. Then, on this Banach space (not algebra), the right regular action is almost isometric with associated weight given by $\Delta_{G}^{1 / p}$.

Also, to simplify the discussion below, we assume that each weight $\mu_{j}^{\alpha}$ is submultiplicative. We start with the simple observation that for any family of tempered weights $\mu$, the extended action (defined in Lemma 4.7) $\hat{\alpha}$ on $\mathcal{B} \underline{\mu}(\mathbb{B}, \mathcal{A})$, commutes with the left regular action $L^{\star}$. This leads us to defined the commuting composite action $\beta:=\hat{\alpha} \circ L^{\star}=L^{\star} \circ \hat{\alpha}$, explicitly given by:

$$
\left(\beta_{g} F\right)\left(g_{0}\right):=\alpha_{g}\left(F\left(g^{-1} g_{0}\right)\right), \quad g, g_{0} \in \mathbb{B}, \quad F \in \mathcal{B} \underline{\mu}(\mathbb{B}, \mathcal{A}) .
$$

Note also that by Lemma 1.12 (ii) and Lemma 4.7 for fixed $g \in \mathbb{B}, \beta_{g}$ sends continuously $\mathcal{B} \underline{\mu}(\mathbb{B}, \mathcal{A})$ to $\mathcal{B} \underline{\nu}(\mathbb{B}, \mathcal{A})$, with $\underline{\nu}=\left\{\mu_{j}^{R_{j}}\right\}$. Thus, in present context of sub-multiplicative weights, $\beta_{g}$ is continuous on $\mathcal{B} \underline{\mu}(\mathbb{B}, \mathcal{A})$. Now observe that for an almost isometric action of a Lie group $G$ on a Fréchet algebra $\mathcal{A}$, the map $\alpha: a \mapsto\left[g \mapsto \alpha_{g}(a)\right]$, is an isometric embedding of $\mathcal{A}^{\infty}$ into $\mathcal{B}^{\mu^{\alpha}}(G, \mathcal{A})$. Indeed for all $j, k \in \mathbb{N}$, we have

$$
\begin{aligned}
\|\alpha(a)\|_{j, k, \underline{\mu}^{\alpha}} & =\sup _{X \in \mathcal{U}_{k}(\mathfrak{g})} \sup _{g \in G} \frac{\|(\tilde{X} \alpha(a))(g)\|_{j}}{\mu_{j}^{\alpha}(g)|X|_{k}} \\
& =\sup _{X \in \mathcal{U}_{k}(\mathfrak{g})} \sup _{g \in G} \frac{\left\|\alpha_{g}\left(X^{\alpha} a\right)\right\|_{j}}{\mu_{j}^{\alpha}(g)|X|_{k}}=\sup _{X \in \mathcal{U}_{k}(\mathfrak{g})} \frac{\left\|X^{\alpha} a\right\|_{j}}{\left|X_{k}\right|}=\|a\|_{j, k} .
\end{aligned}
$$

$\operatorname{By}\left(\mathcal{B}^{\mu^{\alpha}}(\mathbb{B}, \mathcal{A})\right)^{\beta}$, we denote the closed subspace of $\mathcal{B}^{\mu^{\alpha}}(\mathbb{B}, \mathcal{A})$ of fixed points for the action $\beta$. It is then immediate to see that the image of $\mathcal{A}^{\infty}$ under $\alpha$ lies inside $\left(\mathcal{B}^{\mu^{\alpha}}(\mathbb{B}, \mathcal{A})\right)^{\beta}$. Reciprocally, an element $F$ of $\left(\mathcal{B}^{\mu^{\alpha}}(\mathbb{B}, \mathcal{A})\right)^{\beta}$, satisfies $F(g)=$ $\alpha_{g}(F(e))$ for all $g \in \mathbb{B}$, i.e. $F=\alpha(a)$ with $a:=F(e) \in \mathcal{A}$. But by our assumption of almost-isometry and (4.4), we have $\|a\|_{j, k}=\|F\|_{j, k, \underline{\mu}^{\alpha}}$, for all $j, k \in \mathbb{N}$, and thus $a=F(e)$ has to be smooth. This proves that $\alpha: \overline{\mathcal{A}}^{\infty} \rightarrow\left(\mathcal{B}^{\mu^{\alpha}}(\mathbb{B}, \mathcal{A})\right)^{\beta}$ is an isomorphism of Fréchet spaces, which is isometric for each seminorm. Moreover, the map $\alpha$ is an algebra homomorphism. Indeed, by the arguments given in the proof of Theorem 4.8. applied to the case of an almost-isometric action with submultiplicative weights $\underline{\mu}^{\alpha}$, for all $a, b \in \mathcal{A}^{\infty}$ we have the equality

$$
\alpha\left(a \star_{\theta, \vec{\tau}}^{\alpha} b\right)=\alpha(a) \star_{\theta, \vec{\tau}} \alpha(b) \text { in }\left(\mathcal{B} \underline{\mu}^{\alpha}(\mathbb{B}, \mathcal{A})\right)^{\beta},
$$

which also shows that $\left(\mathcal{B}^{\underline{\mu}}(\mathbb{B}, \mathcal{A})\right)^{\beta}$ is an algebra for $\star_{\theta, \vec{\tau}}$. In summary, we have proved the following: 
Proposition 4.13. Let $\theta \in \mathbb{R}^{*}, \vec{\tau} \in \mathbf{\Theta}^{N}$ and let $\left(\mathcal{A}, \alpha, \mu^{\alpha}, \mathbb{B}\right)$ be a Fréchet algebra endowed with a strongly continuous tempered and almost-isometric action of a normal $\mathbf{j}$-group $\mathbb{B}$. Then, we have an isometric isomorphism of Fréchet algebras:

$$
\left(\mathcal{A}^{\infty}, \star_{\theta, \vec{\tau}}^{\alpha}\right) \simeq\left(\left(\mathcal{B}^{\underline{\mu}}(\mathbb{B}, \mathcal{A})\right)^{\beta}, \star_{\theta, \vec{\tau}}\right) .
$$

REMARK 4.14. We stress that the assumption of sub-multiplicativity for the family of weights $\mu^{\alpha}$, associated with the tempered action $\alpha$, is in fact irrelevant in the previous result. However it is unclear to us whether a similar statement holds without the assumption of almost-isometry.

\subsection{Functorial properties of the deformed product}

To conclude with the deformation theory at the level of Fréchet algebras, we establish some functorial properties. We come back to the general setting of a strongly continuous and tempered action $\left(\alpha, \underline{\mu}^{\alpha}\right)$ of a normal $\mathbf{j}$-group $\mathbb{B}$ on a Fréchet algebra $\mathcal{A}$ (i.e. we no longer assume that the action is almost isometric). We start with the question of algebra homomorphisms.

Proposition 4.15. Let $\left(\mathcal{A},\left\{\|\cdot\|_{j}\right\}, \alpha\right),\left(\mathcal{F},\left\{\|\cdot\|_{j}^{\prime}\right\}, \beta\right)$ be two Fréchet algebras endowed with strongly continuous and tempered actions of a normal $\mathbf{j}$-group $\mathbb{B}$ by automorphisms. Let also $T: \mathcal{A} \rightarrow \mathcal{F}$ be a continuous homomorphism which intertwines the actions $\alpha$ and $\beta$. Then for any $\theta \in \mathbb{R}^{*}$ and $\vec{\tau} \in \Theta^{N}$, the map $T$ restricts to a homomorphism from $\left(\mathcal{A}^{\infty}, \star_{\theta, \vec{\tau}}^{\alpha}\right)$ to $\left(\mathcal{F}^{\infty}, \star_{\theta, \vec{\tau}}^{\beta}\right)$.

Proof. Since by assumption $T \circ \alpha=\beta \circ T$, we get for any $P \in \mathcal{U}(\mathfrak{b})$ that $T \circ P^{\alpha}=P^{\beta} \circ T$, which entails that $T$ restricts to a continuous map from $\mathcal{A}^{\infty}$ to $\mathcal{F}^{\infty}$. The remaining part of the statement follows then by Lemma 1.37.

Next, we prove that if a Fréchet algebra is endowed with a continuous involution, then the latter will also define a continuous involution for the deformed product, under the mild condition that $\bar{\tau}(-a)=\tau(a)$. Indeed, the latter implies that

$$
\overline{K_{\theta, \tau}}\left(x_{1}, x_{2}\right)=K_{\theta, \tau}\left(x_{2}, x_{1}\right),
$$

so by Lemma 1.44 we get:

Proposition 4.16. Let $(\mathcal{A}, \alpha)$ be a Fréchet algebras endowed with a strongly continuous tempered action of a normal $\mathbf{j}$-group $\mathbb{B}$. Assuming that for $\theta \in \mathbb{R}^{*}$ and $\vec{\tau} \in \mathbf{\Theta}^{N}$, we have $\bar{\tau}_{j}(-a)=\tau_{j}(a), j=1, \ldots, N$, then any continuous involution of $\mathcal{A}^{\infty}$ is a continuous involution of $\left(\mathcal{A}^{\infty}, \star_{\theta, \vec{\tau}}^{\alpha}\right)$ too.

In a similar way, we deduce from Lemma 1.44 that the deformation is ideal preserving:

Proposition 4.17. Let $(\mathcal{A}, \alpha)$ be a Fréchet algebras endowed with a strongly continuous tempered action of a normal $\mathbf{j}$-group $\mathbb{B}$ and $\theta \in \mathbb{R}^{*}, \vec{\tau} \in \Theta^{N}$. If $\mathcal{I}$ is a closed $\alpha$-invariant ideal of $\mathcal{A}$, then $\mathcal{I}^{\infty}$ is a closed ideal of $\left(\mathcal{A}^{\infty}, \star_{\theta, \vec{\tau}}^{\alpha}\right)$.

We now examine the consequence of the fact that the constant function is the unit of $\left(\mathcal{B}(\mathbb{B}), \star_{\theta, \vec{\tau}}\right)$.

\footnotetext{
${ }^{2}$ In Lemma 7.22 we will see how to suppress this extra condition.
} 
Proposition 4.18. Let $(\mathcal{A}, \alpha)$ be a Fréchet algebras endowed with a strongly continuous and tempered action of a normal $\mathbf{j}$-group $\mathbb{B}$ and $\theta \in \mathbb{R}^{*}, \vec{\tau} \in \mathbf{\Theta}^{N}$. If $a \in \mathcal{A}^{\infty}$ is fixed by the action $\alpha$, then for $b \in \mathcal{A}^{\infty}$, we have

$$
a \star_{\theta, \vec{\tau}}^{\alpha} b=a b, \quad b \star_{\theta, \vec{\tau}}^{\alpha} a=b a .
$$

Proof. This is a consequence of Proposition 3.11 together with the defining relation of the deformed product:

$$
a \star_{\theta, \vec{\tau}}^{\alpha} b=\left(\alpha(a) \star_{\theta, \vec{\tau}} \alpha(b)\right)(e)=\left(a \star_{\theta, \vec{\tau}} \alpha(b)\right)(e)=(a \alpha(b))(e)=a b .
$$

The second equality is entirely similar.

Next, we study the question of the existence of a bounded approximate unit for the Fréchet algebra $\left(\mathcal{A}^{\infty}, \star_{\theta, \vec{\tau}}^{\alpha}\right)$. We recall that a Fréchet algebra $\left(\mathcal{A},\left\{\|\cdot\|_{j}\right\}\right)$ admits a bounded approximate unit if there exists a net $\left\{e_{\lambda}\right\}_{\lambda \in \Lambda}$ of elements of $\mathcal{A}$ such that for any $a \in \mathcal{A}$, the nets $\left\{a e_{\lambda}\right\}_{\lambda \in \Lambda}$ and $\left\{e_{\lambda} a\right\}_{\lambda \in \Lambda}$ converges to $a$ and such that for each $j \in \mathbb{N}$, there exists $C_{j}>0$ such that for every $\lambda \in \Lambda$, we have $\left\|e_{\lambda}\right\|_{j} \leq C_{j}$.

Proposition 4.19. Let $(\mathcal{A}, \alpha)$ be a Fréchet algebra endowed with a strongly continuous and tempered action of a normal $\mathbf{j}$-group $\mathbb{B}$ and such that $\mathcal{A}$ admits a bounded approximate unit. Then for any $\theta \in \mathbb{R}^{*}, \vec{\tau} \in \Theta^{N}$, the Fréchet algebra $\left(\mathcal{A}^{\infty}, \star_{\theta, \vec{\tau}}^{\alpha}\right)$ admits a bounded approximate unit too.

Proof. Let $\left\{f_{\lambda}\right\}$ be a net of bounded approximate units for $\mathcal{A}$, let also $0 \leq$ $\psi \in \mathcal{D}(\mathbb{B})$ be of $L^{1}$-norm one and define

$$
e_{\lambda}:=\int_{\mathbb{B}} \psi(g) \alpha_{g}\left(f_{\lambda}\right) \mathrm{d}_{\mathbb{B}}(g) .
$$

Observe that even if $\left\{f_{\lambda}\right\}$ is not smooth, $\left\{e_{\lambda}\right\}$ is. Indeed, for all $X \in \mathcal{U}(\mathfrak{b})$, we have

$$
X^{\alpha} e_{\lambda}=\int_{\mathbb{B}} \underline{X} \psi(g) \alpha_{g}\left(f_{\lambda}\right) \mathrm{d}_{\mathbb{B}}(g),
$$

and we get for the semi-norms defining the topology of $\mathcal{A}^{\infty}$, with $\underline{\mu}^{\alpha}$ the family of tempered weights associated to the temperedness of the action $\alpha$ :

$$
\begin{aligned}
\left\|e_{\lambda}\right\|_{j, k}=\sup _{X \in \mathcal{U}_{k}(\mathfrak{b})} \frac{\left\|X^{\alpha} e_{\lambda}\right\|_{j}}{|X|_{k}} & \leq \sup _{X \in \mathcal{U}_{k}(\mathfrak{b})} \int_{\mathbb{B}} \underline{\mid X} \psi \mid(g)\left\|\alpha_{g}\left(f_{\lambda}\right)\right\|_{j} \mathrm{~d}_{\mathbb{B}}(g) \\
& \leq \sup _{X \in \mathcal{U}_{k}(\mathfrak{b})} \int_{\mathbb{B}} \frac{|\underline{X} \psi|(g)}{|X|_{k}} \mu_{j}^{\alpha}(g) \mathrm{d} \mathbb{B}(g) \times\left\|f_{\lambda}\right\|_{j} .
\end{aligned}
$$

Hence, the net $\left\{e_{\lambda}\right\}$ belongs to $\mathcal{A}^{\infty}$ and is semi-norm-wise bounded in $\lambda \in \Lambda$ as $\left\|f_{\lambda}\right\|_{j}$ is. Next, we show that it is indeed an approximate unit for $\mathcal{A}^{\infty}$ : Since $\int \psi=1$, we first note that for any $a \in \mathcal{A}$

$e_{\lambda} a-a=\int_{\mathbb{B}} \psi(g)\left(\alpha_{g}\left(f_{\lambda}\right) a-a\right) \mathrm{d}_{\mathbb{B}}(g)=\int_{\mathbb{B}} \psi(g) \alpha_{g}\left(f_{\lambda} \alpha_{g^{-1}}(a)-\alpha_{g^{-1}}(a)\right) \mathrm{d}_{\mathbb{B}}(g)$,

which gives

$$
\left\|e_{\lambda} a-a\right\|_{j} \leq \int_{\mathbb{B}} \psi(g) \mu_{j}^{\alpha}(g)\left\|f_{\lambda} \alpha_{g^{-1}}(a)-\alpha_{g^{-1}}(a)\right\|_{j} \mathrm{~d}_{\mathbb{B}}(g),
$$

which converges to zero because $\left\|f_{\lambda} \alpha_{g^{-1}}(a)-\alpha_{g^{-1}}(a)\right\|_{j}$ does by assumptions and because $\psi$ is compactly supported. The general case is treated recursively exactly as in the proof of Lemma 1.8 (viii). Hence, $\mathcal{A}^{\infty}$ (with its original algebraic structure) admits a bounded approximate unit too. Now, we will prove that a bounded 
approximate unit for $\mathcal{A}^{\infty}$ is also a bounded approximate unit for $\left(\mathcal{A}^{\infty}, \star_{\theta, \vec{\tau}}^{\alpha}\right)$. So, let $\left\{e_{\lambda}\right\}$ be any bounded approximate unit for $\mathcal{A}^{\infty}$. First observe that if we view the product $\star_{\theta, \vec{\tau}}$ as a bilinear map

$\star_{\theta, \vec{\tau}}: \mathcal{B}(\mathbb{B}) \times \mathcal{B}^{\mu}\left(\mathbb{B}, \mathcal{A}^{\infty}\right) \rightarrow \mathcal{B}^{\underline{\nu}}\left(\mathbb{B}, \mathcal{A}^{\infty}\right), \quad \underline{\mu}=\left\{\mu_{j}^{\alpha} \mathfrak{d}_{\mathbb{B}}^{k}\right\}_{j, k \in \mathbb{N}}, \underline{\nu}=\left\{\mu_{j}^{\alpha L_{j}} \mathfrak{d}_{\mathbb{B}}^{k}\right\}_{j, k \in \mathbb{N}}$, a slight adaptation of the arguments of Proposition 3.11 shows that for all $a \in \mathcal{A}^{\infty}$ :

$$
1 \star_{\theta, \vec{\tau}} \alpha(a)=\alpha(a),
$$

where 1 denotes the unit element of $\mathcal{B}(\mathbb{B})$. Combining this with Proposition 4.10 gives the equality in $\mathcal{A}^{\infty}$ :

$$
e_{\lambda} \star_{\theta, \vec{\tau}}^{\alpha} a-a=\int_{\mathbb{B} \times \mathbb{B}} K_{\theta, \vec{\tau}}\left(\alpha\left(e_{\lambda}\right) \otimes \alpha(a)-1 \otimes \alpha(a)\right)
$$

where

$$
\begin{aligned}
\alpha\left(e_{\lambda}\right) & \otimes \alpha(a)-1 \otimes \alpha(a) \\
& :=\left[(x, y) \in \mathbb{B} \times \mathbb{B} \mapsto \alpha_{x}\left(e_{\lambda}\right) \alpha_{y}(a)-\alpha_{y}(a)\right] \in \mathcal{B} \underline{\mu} \otimes \underline{\mu}\left(\mathbb{B} \times \mathbb{B}, \mathcal{A}^{\infty}\right) .
\end{aligned}
$$

But by Proposition 1.36, we know that given $(j, k) \in \mathbb{N}^{2}$, there exist positive integers $\vec{r} \in \mathbb{N}^{4 N}$, such that (where the differential operator $\mathbf{D}_{\vec{r}}$ is given in (1.22)), we have

$$
e_{\lambda} \star_{\theta, \vec{\tau}}^{\alpha} a-a=\int_{\mathbb{B} \times \mathbb{B}} K_{\theta, \vec{\tau}}(x, y) \mathbf{D}_{\vec{r}}\left(\alpha_{x}\left(e_{\lambda}\right) \alpha_{y}(a)-\alpha_{y}(a)\right) \mathrm{d}_{\mathbb{B}}(x) \mathrm{d}_{\mathbb{B}}(y),
$$

with the integral being absolutely convergent for the semi-norm $\|\cdot\|_{j, k}$ of $\mathcal{A}^{\infty}$. Now, take an increasing sequence $\left\{C_{n}\right\}_{n \in \mathbb{N}}$ of relatively compact open subsets in $G$, such that $\lim _{n} C_{n}=G$ and fix $\varepsilon>0$. By the absolute convergence in the semi-norm $\|.\|_{j, k}$ of the integral above and since the net $\left\{e_{\lambda}\right\}_{\lambda \in \Lambda}$ is bounded in the semi-norm $\|\cdot\|_{j, k}$, there exists $n \in \mathbb{N}$ such that

$$
\left\|\int_{\mathbb{B} \times \mathbb{B} \backslash C_{n}} K_{\theta, \vec{\tau}}(x, y) \mathbf{D}_{\vec{r}}\left(\alpha_{x}\left(e_{\lambda}\right) \alpha_{y}(a)-\alpha_{y}(a)\right), \mathrm{d}_{\mathbb{B}}(x) \mathrm{d}_{\mathbb{B}}(y)\right\|_{j, k} \leq \varepsilon .
$$

Moreover, since $\left\{e_{\lambda}\right\}$ is an approximate unit for $\mathcal{A}^{\infty}$, from a compactness argument, we deduce that for any $n \in \mathbb{N}$, we have

$$
\lim _{\lambda}\left\|\int_{C_{n}} K_{\theta, \vec{\tau}}(x, y) \mathbf{D}_{\vec{r}}\left(\alpha_{x}\left(e_{\lambda}\right) \alpha_{y}(a)-\alpha_{y}(a)\right) \mathrm{d}_{\mathbb{B}}(x) \mathrm{d}_{\mathbb{B}}(y)\right\|_{j, k}=0 .
$$

This concludes the proof as the arguments for $a \star_{\theta, \vec{\tau}}^{\alpha} e_{\lambda}$ are similar.

Lastly, we show that the deformation associated with a normal $\mathbf{j}$-group coincides with the iterated deformations of each of its elementary normal $\mathbf{j}$-subgroups.

Proposition 4.20. Let $\mathbb{B}$ be a normal $\mathbf{j}$-group with Pyatetskii-Shapiro decomposition $\mathbb{B}=\mathbb{B}^{\prime} \ltimes \mathbb{S}$, where $\mathbb{B}^{\prime}$ is a normal $\mathbf{j}$-group and $\mathbb{S}$ is an elementary normal $\mathbf{j}$-group. Let $\mathcal{A}$ be a Fréchet algebra endowed with a strongly continuous and tempered action $\left(\alpha, \underline{\mu}^{\alpha}\right)$ of $\mathbb{B}$. Denote by $\alpha^{\mathbb{B}^{\prime}}$ (respectively by $\alpha^{\mathbb{S}}$ ) the restriction of $\alpha$ to $\mathbb{B}^{\prime}$ (respectively to $\mathbb{S}$ ). For $\mathcal{C}$ a subspace of $\mathcal{A}$, denote by $\mathcal{C}_{\mathbb{B}}^{\infty}$ (respectively by $\mathcal{C}_{\mathbb{B}^{\prime}}^{\infty}$, $\left.\mathcal{C}_{\mathbb{S}}^{\infty}\right)$ the set of smooth vectors in $\mathcal{C}$ for the action of $\mathbb{B}$ (respectively of $\mathbb{B}^{\prime}, \mathbb{S}$ ). Then, for $\theta \in \mathbb{R}^{*}$ and $\vec{\tau}=\left(\vec{\tau}^{\prime}, \tau_{1}\right) \in \mathbf{\Theta}^{N+1}$ ( $N$ is the number of elementary factors in $\left.\mathbb{B}^{\prime}\right)$, we have

$$
\left(\left(\mathcal{A}_{\mathbb{S}}^{\infty}, \star_{\theta, \tau_{1}}^{\alpha}\right)_{\mathbb{B}^{\prime}}^{\infty}, \star_{\theta, \vec{\tau}^{\prime}}^{\alpha_{\mathbb{B}^{\prime}}}\right)=\left(\mathcal{A}_{\mathbb{B}}^{\infty}, \star_{\theta, \vec{\tau}}^{\alpha}\right)
$$


Proof. Observe that being the restrictions of a strongly continuous and tempered action, the action $\alpha^{\mathbb{S}}$ of $\mathbb{S}$ on $\mathcal{A}$ is also strongly continuous and tempered. But the action $\alpha^{\mathbb{B}^{\prime}}$ of $\mathbb{B}^{\prime}$ on $\mathcal{A}_{\mathbb{S}}^{\infty}$ is also strongly continuous (which is rather obvious) and tempered. To see that, note that for $g^{\prime} \in \mathbb{B}^{\prime}$ and $a \in \mathcal{A}_{\mathbb{S}}^{\infty}$, we have

$$
\begin{aligned}
\left\|\alpha_{g^{\prime}}^{\mathbb{B}^{\prime}}(a)\right\|_{j, k}=\sup _{X \in \mathcal{U}_{k}(\mathfrak{s})} \frac{\left\|X^{\alpha^{\mathbb{S}}} \alpha_{g^{\prime}}^{\mathbb{B}^{\prime}}(a)\right\|_{j}}{|X|_{k}} & =\sup _{X \in \mathcal{U}_{k}(\mathfrak{s})} \frac{\left\|\alpha_{g^{\prime}}^{\mathbb{B}^{\prime}}\left(\left(\operatorname{Ad}_{g^{\prime}-1} X\right)^{\alpha^{\mathbb{S}}} a\right)\right\|_{j}}{|X|_{k}} \\
& \leq \mu_{j}^{\alpha}\left(g^{\prime}\right) \sup _{X \in \mathcal{U}_{k}(\mathfrak{s})} \frac{\left\|\left(\operatorname{Ad}_{g^{\prime}-1} X\right)^{\alpha^{\mathbb{S}}} a\right\|_{j}}{|X|_{k}}
\end{aligned}
$$

As $\mathbb{B}^{\prime}$ acts on $\mathbb{S}$ by conjugation, it acts on $\mathcal{U}_{k}(\mathfrak{s})$ and by Lemma 1.14, we deduce that

$$
\left\|\alpha_{g^{\prime}}^{\mathbb{B}^{\prime}}(a)\right\|_{j, k} \leq C(k) \mu_{j}^{\alpha}\left(g^{\prime}\right) \mathfrak{d}_{\mathbb{B}}\left(g^{\prime}\right)^{k}\|a\|_{j, k} .
$$

By Lemma 1.5, we have

$$
\left.\mathfrak{d}_{\mathbb{B}}\right|_{\mathbb{B}^{\prime}} \asymp \mathfrak{d}_{\mathbb{B}^{\prime}}+\left[g^{\prime} \mapsto\left(1+\left|\mathbf{R}_{g^{\prime}}\right|^{2}+\left|\mathbf{R}_{g^{\prime-1}}\right|^{2}\right)\right],
$$

and by Lemma 2.34, the extension homomorphism $\mathbf{R}$ is tempered. Hence, the action $\alpha^{\mathbb{B}^{\prime}}$ of $\mathbb{B}^{\prime}$ on $\mathcal{A}_{\mathbb{S}}^{\infty}$ is tempered with associated family of tempered weights given by $\left\{\left.\mu_{j}^{\alpha} \mathfrak{d}_{\mathbb{B}}^{k}\right|_{\mathbb{B}^{\prime}}\right\}_{(j, k) \in \mathbb{N}^{2}}$. Note also that the subspace of smooth vectors for $\mathbb{B}$ coincides with the subspace of smooth vectors for $\mathbb{B}^{\prime}$ within the subspace of smooth vectors for $\mathbb{S}$, i.e.

$$
\mathcal{A}_{\mathbb{B}}^{\infty}=\left(\mathcal{A}_{\mathbb{S}}^{\infty}\right)_{\mathbb{B}^{\prime}}^{\infty}
$$

Indeed, the inclusion $\mathcal{A}_{\mathbb{B}}^{\infty} \subset\left(\mathcal{A}_{\mathbb{S}}^{\infty}\right)_{\mathbb{B}^{\prime}}^{\infty}$ is clear since $a \in\left(\mathcal{A}_{\mathbb{S}}^{\infty}\right)_{\mathbb{B}^{\prime}}^{\infty}$ if and only if for all $X^{\prime} \in \mathcal{U}\left(\mathfrak{b}^{\prime}\right)$, all $X \in \mathcal{U}(\mathfrak{s})$ and all $j \in \mathbb{N}$, we have

$$
\left\|X^{\prime \alpha^{\mathbb{B}^{\prime}}} X^{\alpha^{\mathbb{S}}} a\right\|_{j}<\infty
$$

and $X^{\prime} X \in \mathcal{U}(\mathfrak{b})$. But this also gives the reversed inclusion since $\left[\mathfrak{b}^{\prime}, \mathfrak{s}\right] \subset \mathfrak{s}$, any element of $\mathcal{U}(\mathfrak{b})$ can be written as a finite sum of elements of the form $X^{\prime} X$, with $X^{\prime} \in \mathcal{U}\left(\mathfrak{b}^{\prime}\right)$ and $X \in \mathcal{U}(\mathfrak{s})$.

Next, we show that the action $\alpha^{\mathbb{B}^{\prime}}$ of $\mathbb{B}^{\prime}$ is by automorphisms on the deformed Fréchet algebra $\left(\mathcal{A}_{\mathbb{S}}^{\infty}, \star_{\theta, \tau_{1}}^{\alpha^{\mathbb{S}}}\right)$. First, by Proposition 4.10 and Lemma 1.44, we get with the elements $e_{n} \in \mathcal{D}(\mathbb{S})$ defined in (1.7), $n \in \mathbb{N}$, and for $a, b \in \mathcal{A}_{\mathbb{S}}^{\infty}$ :

$$
\begin{aligned}
a \star_{\theta, \tau_{1}}^{\alpha^{\mathbb{S}}} b & =\int_{\mathbb{S} \times \mathbb{S}} K_{\theta, \tau_{1}}(\alpha(a) \otimes \alpha(b)) \\
& =\lim _{n, m \rightarrow \infty} \int_{\mathbb{S} \times \mathbb{S}} K_{\theta, \tau_{1}}(x, y) e_{n}(x) \alpha_{x}^{\mathbb{S}}(a) e_{m}(y) \alpha_{y}^{\mathbb{S}}(b) \mathrm{d}_{\mathbb{S}}(x) \mathrm{d}_{\mathbb{S}}(y) .
\end{aligned}
$$

Observe also that (4.5) shows that for $g^{\prime} \in \mathbb{B}^{\prime}$ fixed, the operator $\alpha_{g^{\prime}}^{\mathbb{B}^{\prime}}$ is continuous on $\mathcal{A}_{\mathbb{S}}^{\infty}$. From this and the absolute convergence of the integrals in the product $\star_{\theta, \tau_{1}}^{\alpha^{\mathbb{S}}}$ at the level of compactly supported functions, we deduce that for $a, b \in \mathcal{A}_{\mathbb{S}}^{\infty}$ 
and $g^{\prime} \in \mathbb{B}^{\prime}$ :

$$
\begin{aligned}
& \alpha_{g^{\prime}}^{\mathbb{B}^{\prime}}\left(a \star_{\theta, \tau_{1}}^{\alpha^{\mathbb{S}}} b\right)=\lim _{n, m \rightarrow \infty} \alpha_{g^{\prime}}^{\mathbb{B}^{\prime}}\left(\int_{\mathbb{S} \times \mathbb{S}} K_{\theta, \tau_{1}}(x, y) e_{n}(x) \alpha_{x}^{\mathbb{S}}(a) e_{m}(y) \alpha_{y}^{\mathbb{S}}(b) \mathrm{d}_{\mathbb{S}}(x) \mathrm{d}_{\mathbb{S}}(y)\right) \\
& =\lim _{n, m \rightarrow \infty} \int_{\mathbb{S} \times \mathbb{S}} K_{\theta, \tau_{1}}(x, y) e_{n}(x) \alpha_{g^{\prime} x}(a) e_{m}(y) \alpha_{g^{\prime} y}(b) \mathrm{d}_{\mathbb{S}}(x) \mathrm{d}_{\mathbb{S}}(y) \\
& =\lim _{n, m \rightarrow \infty} \int_{\mathbb{S} \times \mathbb{S}} K_{\theta, \tau_{1}}(x, y) e_{n}(x) \alpha_{\mathbf{R}_{g^{\prime}}(x)}^{\mathbb{S}}\left(\alpha_{g^{\prime}}^{\mathbb{B}^{\prime}}(a)\right) e_{m}(y) \alpha_{\mathbf{R}_{g^{\prime}}(y)}^{S}\left(\alpha_{g^{\prime}}^{\mathbb{B}^{\prime}}(b)\right) \mathrm{d}_{\mathbb{S}}(x) \mathrm{d}_{\mathbb{S}}(y) .
\end{aligned}
$$

Remember that

$$
\mathbf{R} \in \operatorname{Hom}\left(\mathbb{B}^{\prime}, \operatorname{Aut}\left(\mathbb{S}, s, \omega^{\mathbb{S}}\right) \cap \operatorname{Sp}\left(V, \omega^{0}\right)\right),
$$

where $\left(V, \omega^{0}\right)$ is the symplectic vector space attached to $\mathbb{S}$. Using the invariance of the two-point kernel and of the left Haar measure under the action of $\operatorname{Sp}\left(V, \omega^{0}\right)=$ $\operatorname{Aut}(\mathbb{S}) \cap \operatorname{Aut}\left(\mathbb{S}, s, \omega^{\mathbb{S}}\right)$, we get:

$$
\begin{aligned}
& \alpha_{g^{\prime}}^{\mathbb{B}^{\prime}}\left(a \star_{\theta, \tau_{1}}^{\alpha^{\mathbb{S}}} b\right) \\
& =\lim _{n, m \rightarrow \infty} \int_{\mathbb{S} \times \mathbb{S}} K_{\theta, \tau_{1}}(x, y) e_{n}(x) \alpha_{x}^{\mathbb{S}}\left(\alpha_{g^{\prime}}^{\mathbb{B}^{\prime}}(a)\right) e_{m}(y) \alpha_{y}^{S}\left(\alpha_{g^{\prime}}^{\mathbb{B}^{\prime}}(b)\right) \mathrm{d}_{\mathbb{S}}(x) \mathrm{d}_{\mathbb{S}}(y) \\
& =\alpha_{g^{\prime}}^{\mathbb{B}^{\prime}}(a) \star_{\theta, \tau_{1}}^{\alpha^{\mathbb{S}}} \alpha_{g^{\prime}}^{\mathbb{B}^{\prime}}(b) .
\end{aligned}
$$

Thus, both Fréchet algebras $\left(\left(\mathcal{A}_{\mathbb{S}}^{\infty}, \star_{\theta, \tau_{1}}^{\alpha}\right)_{\mathbb{B}^{\prime}}^{\infty}, \star_{\theta, \vec{\tau}^{\prime}}^{\alpha^{\prime}}\right)$ and $\left(\mathcal{A}_{\mathbb{B}}^{\infty}, \star_{\theta, \vec{\tau}}^{\alpha}\right)$ are well defined and their underlying sets coincide. It remains to show that their algebraic structures coincide too. But this follows from Proposition 1.39 as the extension homomorphism $\mathbf{R}$ of $\mathbb{B}=\mathbb{B}^{\prime} \ltimes_{\mathbf{R}} \mathbb{S}$ is tempered. 


\section{CHAPTER 5}

\section{Quantization of polarized symplectic symmetric spaces}

In the previous chapters, we defined a deformation of every Fréchet algebra that admits a strongly continuous (and tempered) action of a normal $\mathbf{j}$-group. In particular, the method applies to every $C^{*}$-algebra which the group acts on. However, in that $C^{*}$-case, our procedure does not yet yield, at this stage, pre- $C^{*}$-structures on the deformed algebras. To cure this problem (in the case of an isometric action) we will represent our deformed algebras by bounded operators on an Hilbert space. The present chapter consists in defining these Hilbert space representations.

The construction relies on defining a unitary representation of the normal $\mathbf{j}$ group $\mathbb{B}$ at hand. This unitary representation is obtained as the tensor product of irreducible unitary representations of the symplectic symmetric spaces (cf. (2.5)) underlying the elementary factors $\mathbb{S}_{j}$ in the Pyatetskii-Shapiro decomposition $\mathbb{B}=$ $\left(\mathbb{S}_{N} \ltimes \ldots\right) \ltimes \mathbb{S}_{1}$ of the normal $\mathbf{j}$-group (cf. (2.3) $)$.

At the level of an elementary factor $\mathbb{S}$, the unitary representation Hilbert space will be defined through a variant of Kirillov's orbit method when viewing the symplectic symmetric space $\mathbb{S}$ as a polarized co-adjoint orbit of a central extension of its transvection group (i.e. the subgroup of the automorphism group of the symplectic symmetric space, generated by products of even numbers of geodesic symmetriessee Proposition 5.1 below). However, the construction applies to a much more general situation than the one of elementary normal $\mathbf{j}$-groups: the situation of what we call "elementary local symplectic symmetric spaces". In particular, for these spaces, we will obtain an explicit formula for the composition of symbols (see Proposition 5.55) which will be our main tool to investigate the problem of $C^{*}$ deformations in the next sections. We therefore opted, within the present chapter 5, to start with presenting this more general situation in the sections 5.1 to 5.6, and then to later pass to the particular case of elementary normal $\mathbf{j}$-groups in chapter 6.

Once the elementary case is treated, one then needs to pass to the case of a normal $\mathbf{j}$-group $\mathbb{B}$. In that case, as already mentioned above, the Hilbert space $\mathcal{H}$ will consist in the tensor product of the Hilbert spaces $\mathcal{H}_{j}$ representing its elementary factors $\mathbb{S}_{j}$. In order to define the $\mathbb{B}$-action on the tensor product Hilbert space $\mathcal{H}$, we will need to represent every elementary group factor $\mathbb{S}_{j}$ on each $\mathcal{H}_{k}$ for every $k$ less than or equal to $j$. The issue here is that the transvection group of $\mathbb{S}_{k}$ does not generally contain $\mathbb{S}_{j}$ as a subgroup. However, we will show that $\mathbb{S}_{j}$ injects into the subgroup of the automorphism group Aut $\left(\mathbb{S}_{k}\right)$ of $\mathbb{S}_{k}$ that preserves Kirillov's polarization (see Proposition 6.26 ) on $\mathbb{S}_{k}$. The latter property will be shown, already in the more general case of local symplectic symmetric spaces, to be sufficient to extend the action of $\mathbb{S}_{k}$ on $\mathcal{H}_{k}$ to an action of $\mathbb{S}_{j} \ltimes \mathbb{S}_{k}$ on $\mathcal{H}_{j} \otimes \mathcal{H}_{k}$ 
(see section 5.7 below). Iterating this result will then lead to our definition of the unitary representation of $\mathbb{B}$ on $\mathcal{H}$ and its associated symbol calculus.

At the level of an elementary normal $\mathbf{j}$-group $\mathbb{S}$, the above mentioned quantization map (allowing to represent our algebras by bounded operators), realizes the program of the construction of 'covariant Moyal quantizers' in the sense of $\mathbf{1 2}$ (see also [18, Section 3.5]). Such an object is of the following nature: Let $(M, \omega)$ be a symplectic manifold and let $G$ be a Lie subgroup of the symplectomorphisms group $\operatorname{Symp}(M, \omega)$, together with $(\mathcal{H}, U)$, a Hilbert space carrying a unitary representation of $G$. Then, a covariant Moyal quantizer, is a family $\{\Omega(x)\}_{x \in M}$ of densely defined self-adjoint operators on $\mathcal{H}$ such that

$$
\begin{aligned}
U(g) \Omega(x) U(g)^{\star} & =\Omega(g . x), \quad \forall(g, x) \in G \times M, \\
\operatorname{Tr}(\Omega(x)) & =1, \quad \forall x \in M, \\
\operatorname{Tr}(\Omega(x) \Omega(y)) & =\delta_{x}(y), \quad \forall(x, y) \in M \times M,
\end{aligned}
$$

where the traces have to be understood in the distributional sense and where $\delta_{x}(y)$ is a shorthand for the reproducing kernel of the Liouville measure on $M$. Associated to a Moyal quantizer, there are quantization and dequantization maps, respectively given, on suitable domains and with $d \mu$ the Liouville measure on $M$, by

$$
\Omega: f \mapsto \int_{M} f(x) \Omega(x) d \mu(x),
$$

and by

$$
\sigma: A \mapsto \operatorname{Tr}(\Omega(x) A)
$$

Then, condition (5.1) ensures that both quantization and dequantization maps are $G$-equivariant. Condition (5.2) is a normalization condition and condition (5.3) says that $\Omega$ and $\sigma$ are formal inverses of each other. At the non-formal level, condition (5.3) together with self-adjointness of the quantizers, implies that $\Omega$ is a unitary operator from $L^{2}(M)$ to $\mathcal{L}^{2}(\mathcal{H})$, the Hilbert space of Hilbert-Schmidt operators on $\mathcal{H}$. Transporting the algebraic structure from $\mathcal{L}^{2}(\mathcal{H})$ to $L^{2}(M)$, one therefore obtains a non-formal and $G$-equivariant star-product at the level of square integrable functions:

$$
f_{1} \star f_{2}:=\sigma\left(\Omega\left(f_{1}\right) \Omega\left(f_{2}\right)\right) .
$$

This non-formal star-product turns to be a tri-kernel product, with distributional kernel computable as it is given by the trace of three quantizers:

$$
f_{1} \star f_{2}(x)=\int_{M \times M} f_{1}\left(x_{1}\right) f_{2}\left(x_{2}\right) \operatorname{Tr}\left(\Omega(x) \Omega\left(x_{1}\right) \Omega\left(x_{2}\right)\right) d \mu\left(x_{1}\right) d \mu\left(x_{2}\right) .
$$

To construct such a quantizer for an elementary local symplectic symmetric spaces, we essentially follow the pioneer ideas of Unterberger [33] and recast them in a much more general geometric context. Note that, however, in the work of Unterberger the condition (5.3) does not hold but, as we will see in the present chapter, is restorable by a minor modification of his construction. This condition is also called the 'traciality property' as it eventually allows to prove that:

$$
\int_{M} f_{1} \star f_{2}(x) d \mu(x)=\int_{M} f_{1}(x) f_{2}(x) d \mu(x), \quad \forall f_{1}, f_{2} \in L^{2}(M) .
$$

Traciality is a property not shared by every quantization map (for example its fails for the coherent-state quantization) and proved to be fundamental in the work of 
Gracia-Bondía and Várilly. For instance, in [12, they were able to construct (and to prove uniqueness of) an equivariant quantization map on the (regular) orbits of the Poincaré group in $3+1$ dimensions solely from the axioms (5.1), (5.2), (5.3).

\subsection{Polarized symplectic symmetric spaces}

In this section, we introduce a particular class of symplectic symmetric spaces (called hereafter "polarized") for which we will be able to define a symmetric space variant of Kirillov's orbit method (see the next section). Within the present section, after defining polarized symplectic symmetric spaces, we associate to every such space an algebraic object (its "polarization quadruple") on which we will later base our unitary representation.

Let $(M, s, \omega)$ be a symplectic symmetric space and let $\operatorname{Aut}(M, s, \omega)$ be its automorphism group and $\mathfrak{a u t}(M, s, \omega)$ its derivation algebra (see Definition 2.7 and Proposition 2.8). Choose a base point $o$ in $M$. Then the conjugation by the symmetry at $o$ yields an involutive automorphism of the automorphism group:

$$
\sigma: \operatorname{Aut}(M, s, \omega) \rightarrow \operatorname{Aut}(M, s, \omega), \quad g \mapsto s_{o} \circ g \circ s_{o}=: \sigma(g) .
$$

The following result is a simple adaptation to the symplectic situation of a standard fact for general symmetric spaces [21]:

Proposition 5.1. The smallest subgroup $G(M)$ of $\operatorname{Aut}(M, s, \omega)$ that is stable under $\sigma$ and that acts transitively on $M$ is a Lie subgroup of $\operatorname{Aut}(M, s, \omega)$. It coincides with group generated by products of an even number of symmetries:

$$
G(M)=\operatorname{gr}\left\{s_{x} \circ s_{y} \mid x, y \in M\right\} .
$$

The group $G(M)$ is called the transvection group of $M$.

We now come to the notion of polarized symplectic symmetric spaces:

Definition 5.2. A symplectic symmetric space $(M, \omega, s)$ is said to be polarizable if it admits a $G(M)$-invariant Lagrangian tangent distribution. A choice of such a transvection-invariant distribution $\tilde{W} \subset T M$ determines a polarization of $M$, in which case one speaks about a polarized symplectic symmetric space.

The infinitesimal version of the notion of symplectic symmetric space is given in the two following definitions 1 (see $[\mathbf{1}, \mathbf{5}$ ):

DEFINITION 5.3. A symplectic involutive Lie algebra (shortly a "siLa") is a triple $(\mathfrak{g}, \sigma, \varpi)$ where $(\mathfrak{g}, \sigma)$ is an involutive Lie algebra (shortly an "iLa") i.e. $\mathfrak{g}$ is a finite dimensional real Lie algebra and $\sigma$ is an involutive automorphism of $\mathfrak{g}$, and, where $\varpi \in \Lambda^{2} \mathfrak{g}^{\star}$ is a Chevalley two-cocycle on $\mathfrak{g}$ (valued in the trivial representation on $\mathbb{R}$ ) such that, denoting by

$$
\mathfrak{g}=\mathfrak{k} \oplus \mathfrak{p},
$$

the \pm 1 -eigenspace decomposition of $\mathfrak{g}$ associated to the involution $\sigma=: \mathfrak{i d} d_{\mathfrak{k}} \oplus\left(-i d_{\mathfrak{p}}\right)$, the cocycle $\varpi$ contains $\mathfrak{k}$ in its radical and restricts to $\mathfrak{p} \times \mathfrak{p}$ as a non-degenerate two-form. A morphism between two such siLa's is a Lie algebra homomorphism which intertwines both involutions and two-cocycles.

Definition 5.4. A transvection symplectic triple is a siLa $(\mathfrak{g}, \sigma, \varpi)$ where

\footnotetext{
${ }^{1}$ Involutions $\sigma$ are denoted the same way either at the Lie group or Lie algebra level.
} 
(i) the action of $\mathfrak{k}$ on $\mathfrak{p}$ is faithful, and

(ii) $[\mathfrak{p}, \mathfrak{p}]=\mathfrak{k}$.

LEMma 5.5. Every symplectic symmetric space determines a transvection symplectic triple.

Proof. Let $(M, s, \omega)$ be a symplectic symmetric space. Define a transvection symplectic triple $(\mathfrak{g}, \sigma, \varpi)$ as follows: $\mathfrak{g}$ is the Lie algebra of the transvection group $G(M), \sigma$ is the restriction to $\mathfrak{g}$ of the differential of the involution (5.4) and $\varpi=$ $\pi_{\star e}^{\star} \omega_{o}$ is the pullback of $\omega$ at the base point $o$ by the differential $\pi_{\star e}: \mathfrak{g} \rightarrow T_{o} M$ of the projection $\pi: G(M) \rightarrow M, g \mapsto g$.o. See [1] for the details.

Defining the notion of isomorphism in the obvious way, one knows from [1,5] the following result. It is a symplectic adaptation of the classical analogue for general affine symmetric spaces [21.

Proposition 5.6. The correspondence $(M, s, \omega) \mapsto(\mathfrak{g}, \sigma, \varpi)$ described above induces a bijection between the isomorphism classes of simply connected symplectic symmetric spaces and the isomorphism classes of transvection symplectic triples.

In the above statement, the "reverse direction" (i.e. $(\mathfrak{g}, \sigma, \varpi) \mapsto(M, s, \omega)$ ) is obtained as follows. One starts by considering the (abstract) connected simply connected Lie group $G$ whose Lie algebra is $\mathfrak{g}$. Then the automorphism $\sigma$ of $\mathfrak{g}$ uniquely determines an automorphism of $G$, again denoted by $\sigma$. The connected component $K$ of the subgroup of $G$ constituted by the elements that are fixed by $\sigma$ is then automatically a closed subgroup of $G$. The coset space $M:=G / K$ is then naturally a connected smooth manifold which is also simply connected consequently to the connectedness of $K$ and simple connectedness of $G$. One then check that the formulae

$$
s_{g K}\left(g^{\prime} K\right):=g \sigma\left(g^{-1} g^{\prime}\right) K,
$$

define a structure of symmetric space $(M, s)$ in the sense of Loos. The Lie algebra of $K$ then coincides with $\mathfrak{k}$, implying a natural isomorphism $\mathfrak{p} \rightarrow T_{K}(M)$. The latter yields a Lie algebra homomorphism from the original iLa $(\mathfrak{g}, \sigma)$ to the transvection iLa of $(M, s)$. This homomorphism turns out to be an isomorphism due to the above conditions (i) and (ii) imposed on $(\mathfrak{g}, \sigma)$. Note that from the above exposition, one extracts

Lemma 5.7. Every iLa determines a simply connected symmetric space.

Note also that two non-isomorphic iLa's (when non-transvection) could determine the same symmetric space. More precisely:

Definition 5.8. Let $\left(\mathfrak{g}_{j}, \sigma_{j}\right)(j=1,2)$ be two iLa's with associated simply connected symmetric spaces denoted by $\left(M_{j}, s_{j}\right)$ respectively. One says that they determine the same simply connected symmetric space if $M_{1}$ and $M_{2}$ are isomorphic as symmetric spaces.

Of course, one has an analogous definition in the symplectic case.

Now given a siLa $(\mathfrak{g}, \sigma, \varpi)$, the two-cocycle $\varpi$ is generally not exact, or equivalently, the symplectic action of the transvection group on the symplectic symmetric space is not Hamiltonian (see [1, $\mathbf{5}$ ). However, it is always possible to centrally extend the transvection group in such a way that the extended group acts on $M$ 
in a Hamiltonian way. The associated moment mapping then is a symplectic equivariant covering onto a co-adjoint orbit, in accordance with the classical general result for Hamiltonian homogeneous symplectic spaces [31. The situation we consider in the present article concerns such non-exact transvection triples underlying polarized symplectic symmetric spaces.

Lemma 5.9. Let $(M, s, \omega)$ be a symplectic symmetric space, polarized by a transvection-invariant Lagrangian distribution $\tilde{W} \subset T M$. These data correspond (via the correspondence of Proposition 5.6) to a k-invariant Lagrangian subspace $W$ in $\mathfrak{p}$.

Proof. Under the linear isomorphism $\left.\pi_{\star e}\right|_{\mathfrak{p}}: \mathfrak{p} \rightarrow T_{o} M$ the subspace $\tilde{W}_{o}$ of $T_{o} M$ corresponds to a Lagrangian subspace $W$ of the symplectic vector space $(\mathfrak{p}, \varpi)$.

According to the previous Lemma, we use the following terminology:

Definition 5.10. A siLa $(\mathfrak{g}, \sigma, \varpi)$ is called polarized if it is endowed with $W$, a $\mathfrak{k}$-invariant Lagrangian subspace of $(\mathfrak{p}, \varpi)$.

Let $(\mathfrak{g}, \sigma, \varpi)$ be a non-exact transvection symplectic triple (i.e. the Chevalley two-cocycle $\varpi$ is not exact) polarized by a Lagrangian subspace $W \subset \mathfrak{p}$. Let us consider $\mathfrak{D}$, the algebra of $W$-preserving symplectic endomorphisms of $\mathfrak{p}$. Note that the faithfulness condition (i) of Definition 5.4 implies the inclusion $\mathfrak{k} \subset \mathfrak{D}$. The vector space $\mathfrak{D} \oplus \mathfrak{p}$ then naturally carries a structure of Lie algebra (containing $\mathfrak{g})$ that underlies a siLa. We centrally extend the latter in order to define a new siLa:

$$
\mathfrak{L}:=\mathfrak{D} \oplus \mathfrak{p} \oplus \mathbb{R} Z
$$

with table given by

$$
[X, Y]_{\mathfrak{L}}:=[X, Y]+\varpi(X, Y) Z, \quad[X, Z]_{\mathfrak{L}}:=0, \quad \forall X, Y \in \mathfrak{D} \oplus \mathfrak{p},
$$

where $[.,$.$] denotes the Lie bracket in \mathfrak{D} \oplus \mathfrak{p}$ and where we have extended the 2 -form $\varpi$ on $\mathfrak{p}$ to a 2 -form on the entire Lie algebra $\mathfrak{D} \oplus \mathfrak{p}$ by zero on $\mathfrak{D}$.

Lemma 5.11. Let $(\mathfrak{g}, \sigma, \varpi)$ be a non-exact polarized transvection symplectic triple. Within the notations given above, consider the element $\xi \in \mathfrak{L}^{\star}$ defined by

$$
\langle\xi, Z\rangle=1,\left.\quad \xi\right|_{\mathfrak{D} \oplus \mathfrak{p}}=0
$$

Define moreover:

$$
\tilde{\mathfrak{D}}:=\mathfrak{D} \oplus \mathbb{R} Z, \quad \sigma_{\mathfrak{L}}:=\mathrm{id}_{\mathfrak{\mathfrak { D }}} \oplus\left(-i \mathrm{~d}_{\mathfrak{p}}\right)
$$

and

$$
\tilde{\mathfrak{g}}=\mathfrak{g} \oplus \mathbb{R} Z, \quad \tilde{\mathfrak{k}}=\mathfrak{k} \oplus \mathbb{R} Z, \quad \tilde{\sigma}:=i \mathrm{~d}_{\tilde{\mathfrak{k}}} \oplus\left(-i \mathrm{~d}_{\mathfrak{p}}\right) .
$$

Then, the triples $\left(\mathfrak{L}, \sigma_{\mathfrak{L}}, \delta \xi\right)$ and $\left(\tilde{\mathfrak{g}}, \tilde{\sigma},\left.\delta \xi\right|_{\tilde{\mathfrak{g}}}\right)$ are exact siLa's.

Proof. We give the proof for the first triple $\left(\mathfrak{L}, \sigma_{\mathfrak{L}}, \delta \xi\right)$ only, the second case being handled in a similar way. Since for $X, Y \in \mathfrak{p}$, we have $[X, Y] \in \mathfrak{k} \subset \mathfrak{D}$, we get

$$
\delta \xi(X, Y)=\left\langle\xi,[X, Y]_{\mathfrak{L}}\right\rangle=\langle\xi,[X, Y]+\varpi(X, Y) Z\rangle=\varpi(X, Y),
$$

which at once proves closedness, non-degeneracy and $\tilde{\mathfrak{D}}$-invariance of the 2 -form $\delta \xi$ on $\mathfrak{p}$. 
REMARK 5.12. The exact siLa's $\left(\mathfrak{L}, \sigma_{\mathfrak{L}}, \delta \xi\right)$ and $\left(\tilde{\mathfrak{g}}, \tilde{\sigma},\left.\delta \xi\right|_{\tilde{\mathfrak{g}}}\right)$ and the non-exact $\operatorname{siLa}(\mathfrak{g}, \sigma, \varpi)$ all three determine the same simply connected symplectic symmetric space.

Definition 5.13. Given an exact $\operatorname{siLa}(\mathfrak{g}, \sigma, \varpi)$ (i.e. $\varpi=\delta \xi$ for an element $\left.\xi \in \mathfrak{g}^{\star}\right)$, by a polarization affiliated to $\xi$, we mean a $\sigma$-stable Lie subalgebra $\mathfrak{b}$ of $\mathfrak{g}$ containing $\mathfrak{k}$ and maximal for the property of being isotropic with respect to the two-form $\varpi$.

The following statement is classical (see e.g. [21]).

LEMMA 5.14. Let $(G, \sigma)$ be a connected involutive Lie group i.e. a connected Lie group $G$ equipped with an involutive automorphism $\sigma$. Let us denote by $K$ the connected component of the subgroup of $G$ constituted by the $\sigma$-fixed elements. Then $K$ must be closed and Formula (5.5) defines a structure of symmetric space on the quotient manifold $M=G / K$.

Note that in this slightly more general situation, $M$ need not necessarily be simply connected.

Within the above setting let us denote by $(\mathfrak{g}, \sigma)$ the involutive Lie algebra associated to $(G, \sigma)$. Let us furthermore assume that it underlies an exact siLa with polarization $\mathfrak{b}$ as in Definition 5.13. In that context, $M$ automatically becomes a polarized symplectic symmetric space. Denote by $B:=\exp \{\mathfrak{b}\}$ the analytic (i.e. connected) Lie subgroup of $G$ with Lie algebra $\mathfrak{b}$. One has $K \subset B$ and we will always assume $B$ to be closed in $G$. Since $B$ is stable under $\sigma$, the coset space $G / B$ admits the following natural family of involutions $\underline{\sigma}$ :

$$
M \times G / B \rightarrow G / B, \quad\left(g K, g_{0} B\right) \mapsto \underline{\sigma}_{g K}\left(g_{0} B\right):=g \sigma\left(g^{-1} g_{0}\right) B .
$$

DEFinition 5.15. With the same notations as above, the quadruple $(G, \sigma, \xi, B)$ is called a polarization quadruple. Its infinitesimal version $(\mathfrak{g}, \sigma, \xi, \mathfrak{b})$ is called the associated infinitesimal polarization quadruple. A morphism between two polarization quadruples $\left(G_{j}, \sigma_{j}, \xi_{j}, B_{j}\right), j=1,2$, is defined as a Lie group homomorphism

$$
\phi: G_{1} \rightarrow G_{2}
$$

that intertwines the involutions, such that $\phi\left(B_{1}\right) \subset B_{2}$ and such that, denoting again by $\phi$ its differential at the unit element, one has $\phi^{\star} \xi_{2}=\xi_{1}$.

The map (5.6) corresponds to an 'action' of the symmetric space $M=G / K$ on the manifold $G / B$. The following result is a consequence of immediate computations.

Lemma 5.16. Let $(G, \sigma, \xi, B)$ be a polarization quadruple. Then, the following properties hold for all $x$ and $y$ in $M$ :

$$
\underline{\sigma}_{x}^{2}=\operatorname{Id}_{G / B}, \quad \underline{\sigma}_{x} \circ \underline{\sigma}_{y} \circ \underline{\sigma}_{x}=\underline{\sigma}_{s(x, y)} .
$$

Moreover, we have the G-equivariance property:

$$
g \circ \underline{\sigma}_{x} \circ g^{-1}=\underline{\sigma}_{g . x}, \quad \forall g \in G, \forall x \in M .
$$

We also observe that under mild conditions on the modular functions of $G$ and $B$, a $G$-invariant and $M$-invariant measure always exists on the manifold $G / B$ : 
Lemma 5.17. Let $(G, \sigma)$ be an involutive Lie group and $B$ a $\sigma$-stable closed subgroup of $G$ such that the modular function of $B$ coincides with the restriction to $B$ of the modular function of $G$. Then, there exists a (unique up to normalization) Borelian measure $\mathrm{d}_{G / B}$ on the manifold $G / B$ which is both invariant under $G$ and under the action of $M=G / K$ given in (5.6).

Proof. Under the closedness condition of $B$ and under the coincidence assumption for the modular functions, it is well known that there exists a (unique up to normalization) $G$-invariant Borelian measure $\mathrm{d}_{G / B}^{o}$ on $G / B$. Now, define $\mathrm{d}_{G / B}:=\mathrm{d}_{G / B}^{o}+\underline{\sigma}_{K}^{\star} \mathrm{d}_{G / B}^{o}$. As $\underline{\sigma}_{K}$ is an involution, the latter measure is $\underline{\sigma}_{K^{-}}$ invariant. Moreover, from $L_{g}^{\star} \circ \underline{\sigma}_{K}^{\star}=\underline{\sigma}_{K}^{\star} \circ L_{\sigma(g)}^{\star}$ on $G / B$ for all $g \in G$, we deduce that $\mathrm{d}_{G / B}$ is also $G$-invariant. By uniqueness of $\mathrm{d}_{G / B}^{o}$, the latter is a multiple of the former. To conclude with the $M$-invariance, it suffices to observe that for all $g \in G$, we have $\underline{\sigma}_{g K}^{\star}=L_{g}^{\star} \circ \underline{\sigma}_{K}^{\star} \circ L_{g^{-1}}^{\star}$.

We end this section by constructing two canonical exact polarization quadruples out of a non-exact transvection triple. We omit the proof which is immediate.

Proposition 5.18. Let $(\mathfrak{g}, \sigma, \varpi)$ be a non-exact transvection symplectic triple polarized by $W \subset \mathfrak{p}$. Within the context of Lemma [5.11, we set $\mathfrak{B}:=\tilde{\mathfrak{D}} \oplus W$ and $\mathfrak{b}:=\tilde{\mathfrak{k}} \oplus W$. Then the quadruples $\left(\mathfrak{L}, \sigma_{\mathfrak{L}}, \xi, \mathfrak{B}\right)$ and $\left(\tilde{\mathfrak{g}}, \tilde{\sigma},\left.\xi\right|_{\tilde{\mathfrak{g}}}, \mathfrak{b}\right)$ are polarization quadruples.

The latter observation leads us to introduce the following terminology:

Definition 5.19. The polarization quadruple $\left(\mathfrak{L}, \sigma_{\mathfrak{L}}, \xi, \mathfrak{B}\right)$ associated to a nonexact polarized transvection triple $(\mathfrak{g}, \sigma, \varpi)$, is called the (infinitesimal) full polarization quadruple. The sub-quadruple $\left(\tilde{\mathfrak{g}}, \tilde{\sigma},\left.\xi\right|_{\tilde{\mathfrak{g}}}, \mathfrak{b}\right)$ is called the associated (infinitesimal) transvection quadruple.

In the sequel, we will denote by $\mathbb{L}$ the connected, simply connected Lie group with Lie algebra $\mathfrak{L}$ and we will consider the connected Lie subgroup $\tilde{G}$ of $\mathbb{L}$ tangent to $\tilde{\mathfrak{g}}$. We will denote by $\mathbf{B}$ (respectively $B$ ) the connected Lie subgroup of $\mathbb{L}$ (respectively of $\tilde{G}$ ) associated to $\mathfrak{B}$ (respectively to $\mathfrak{b}$ ).

We summarize the present section by the following

Proposition 5.20. $\quad$ (i) Every symplectic symmetric space uniquely determines a transvection symplectic triple (cf. Definition 5.4).

(ii) Every siLa (cf. Definition [5.3) uniquely determines a simply connected symplectic symmetric space.

(iii) In the simply connected and transvection case, the correspondences mentioned in items (i) and (ii) are inverse to one another.

(iv) Every non-exact transvection symplectic triple $(\mathfrak{g}, \sigma, \varpi)$ (cf. Definition 5.4 with non-exact two-cocycle) uniquely determines a pair of exact polarization quadruples: the associated infinitesimal full polarization quadruple $\left(\mathfrak{L}, \sigma_{\mathfrak{L}}, \xi, \mathfrak{B}\right)$ and its transvection sub-quadruple $\left(\tilde{\mathfrak{g}}, \tilde{\sigma},\left.\xi\right|_{\mathfrak{\mathfrak { g }}}, \mathfrak{b}\right)$ (cf. Proposition 5.18 and Definition 5.19).

(v) The three siLa's involved in item (iv): $(\mathfrak{g}, \sigma, \varpi),\left(\tilde{\mathfrak{g}}, \tilde{\sigma},\left.\delta \xi\right|_{\tilde{\mathfrak{g}}}\right)$ and $\left(\mathfrak{L}, \sigma_{\mathfrak{L}}, \delta \xi\right)$ all determine the same simply connected polarized symplectic symmetric space (cf. Definition 5.8). 


\subsection{Unitary representations of symmetric spaces}

In this section, we fix $(\mathfrak{g}, \sigma, \varpi)$ a non-exact polarized transvection triple, to which we associate a (connected and simply connected) transvection quadruple $(\tilde{G}, \tilde{\sigma}, \xi, B)$, according to the construction underlying Definition 5.19. We start with the following pre-quantization condition: in the sequel we will always assume that the character $\left.\xi\right|_{\mathfrak{b}}: \mathfrak{b} \rightarrow \mathbb{R}$ exponentiates to $B$ as a unitary character

$$
\chi: B \rightarrow U(1), \quad b \mapsto \chi(b) .
$$

By this we mean that we assume the existence of a Lie group homomorphism $\chi$ whose differential at the identity coincides with $\left.\xi\right|_{\mathfrak{b}}$. Note that then, the character is automatically fixed by the restriction to $B$ of the involution:

$$
\sigma^{\star} \chi=\chi \text {. }
$$

Of course, the pre-quantization condition is satisfied when the group $B$ is exponential, as it will be the case for Pyatetskii-Shapiro's elementary normal $\mathbf{j}$-groups:

$$
\chi(b):=e^{i\langle\xi, \log (b)\rangle}, \quad b \in B .
$$

LEMma 5.21. Let $(\tilde{\mathfrak{g}}, \tilde{\sigma}, \xi, \mathfrak{b})$ be the transvection quadruple of a non-exact transvection triple $(\mathfrak{g}, \sigma, \varpi)$ such that $B$ is exponential. Then, the pre-quantization condition is satisfied.

Proof. Since $B$ is exponential, by the BCH formula, the statement will follow from $\xi\left([\mathfrak{b}, \mathfrak{b}]_{\tilde{g}}\right)=0$. By construction of $\xi$ (see Definition [5.11), this will follow if the $Z$-component of $[\mathfrak{b}, \mathfrak{b}]_{\tilde{g}}$ vanishes. But the latter reads $\varpi(\mathfrak{b}, \mathfrak{b}) Z$ which reduces to zero by Definition 5.13 of a polarization quadruple and by Proposition 5.18 which shows that $(\tilde{g}, \tilde{\sigma}, \xi, \mathfrak{b})$ is indeed a polarization quadruple.

We then form the line bundle:

$$
E_{\chi}:=\tilde{G} \times_{\chi} \mathbb{C} \rightarrow \tilde{G} / B,
$$

and consider the associated induced representation of $\tilde{G}$ on the smooth sections $\Gamma^{\infty}\left(E_{\chi}\right)$. We will denote the latter representation by $U_{\chi}$. Identifying as usual $\Gamma^{\infty}\left(E_{\chi}\right)$ with the space of $B$-equivariant functions:

$$
\begin{aligned}
\Gamma^{\infty}\left(E_{\chi}\right) & \simeq C^{\infty}(\tilde{G})^{B} \\
& :=\left\{\hat{\varphi} \in C^{\infty}(\tilde{G}) \mid \hat{\varphi}(g b)=\bar{\chi}(b) \hat{\varphi}(g), \forall b \in B, \forall g \in \tilde{G}\right\},
\end{aligned}
$$

the representation $U_{\chi}$ is given by the restriction to $C^{\infty}(\tilde{G})^{B}$ of the left-regular representation:

$$
\left[U_{\chi}(g) \tilde{\varphi}\right]^{\wedge}\left(g^{\prime}\right):=\hat{\varphi}\left(g^{-1} g^{\prime}\right), \quad \forall \tilde{\varphi} \in \Gamma^{\infty}\left(E_{\chi}\right) .
$$

We endow the line bundle $E_{\chi}$ with the Hermitian structure, defined in terms of the identification (5.8) by:

$$
h_{g B}\left(\tilde{\varphi}_{1}, \tilde{\varphi}_{2}\right):=\overline{\hat{\varphi}_{1}(g)} \hat{\varphi}_{2}(g), \quad \forall \tilde{\varphi}_{1}, \tilde{\varphi}_{2} \in \Gamma^{\infty}\left(E_{\chi}\right), \quad g B \in \tilde{G} / B .
$$

We make the assumption that the modular function of $B$ coincides with the restriction to $B$ of the modular function of $\tilde{G}$. By Lemma 5.17 this condition implies the existence of a $\tilde{G}$-invariant and $\underline{\sigma}_{\tilde{K}}$-invariant Borelian measure $\mathrm{d}_{\tilde{G} / B}$ on $\tilde{G} / B$. Here, $\underline{\sigma}_{\tilde{K}}: \tilde{G} / B \rightarrow \tilde{G} / B, g B \mapsto \tilde{\sigma}(g) B$ is the involutive diffeomorphism given in (5.6) for the involutive pair $(\tilde{G}, \tilde{\sigma})$ and subgroup $B$, underlying the transvection quadruple 
$(\tilde{G}, \tilde{\sigma}, \xi, B)$. We then let $\mathcal{H}_{\chi}$ be the Hilbert space completion of $\Gamma_{c}^{\infty}\left(E_{\chi}\right)$ for the inner product:

$$
\left\langle\tilde{\varphi}_{1}, \tilde{\varphi}_{2}\right\rangle:=\int_{\tilde{G} / B} h_{g B}\left(\tilde{\varphi}_{1}, \tilde{\varphi}_{2}\right) \mathrm{d}_{\tilde{G} / B}(g B) .
$$

Of course, the induced representation $U_{\chi}$ of $\tilde{G}$ then naturally acts on $\mathcal{H}_{\chi}$ by unitary operators. Now observe that the $\tilde{\sigma}$-invariance of character $\chi$, implies that the pull back under $\tilde{\sigma}$ of an equivariant function is again equivariant. Therefore, we get a linear involution:

$$
\Sigma: \mathcal{H}_{\chi} \rightarrow \mathcal{H}_{\chi}, \quad[\Sigma \tilde{\varphi}]^{\wedge}:=\tilde{\sigma}^{\star} \hat{\varphi} .
$$

Also, the $\underline{\sigma}_{\tilde{K}}$-invariance of the measure $\mathrm{d}_{\tilde{G} / B}$ implies:

$$
\begin{aligned}
\left\langle\Sigma \tilde{\varphi}_{1}, \Sigma \tilde{\varphi}_{2}\right\rangle & =\int_{\tilde{G} / B} h_{g B}\left(\Sigma \tilde{\varphi}_{1}, \Sigma \tilde{\varphi}_{2}\right) \mathrm{d}_{\tilde{G} / B}(g B) \\
& =\int_{\tilde{G} / B} h_{\underline{\sigma}_{\tilde{K}}(g B)}\left(\tilde{\varphi}_{1}, \tilde{\varphi}_{2}\right) \mathrm{d}_{\tilde{G} / B}(g B)=\left\langle\tilde{\varphi}_{1}, \tilde{\varphi}_{2}\right\rangle,
\end{aligned}
$$

for all $\tilde{\varphi}_{1}, \tilde{\varphi}_{2} \in \mathcal{H}_{\chi}$, showing that $\Sigma$ is not only involutive but also self-adjoint. Thus the element $\Sigma$ belongs to $\mathcal{U}_{s a}\left(\mathcal{H}_{\chi}\right)$, the collection of unitary and self-adjoint operators on $\mathcal{H}_{\chi}$. When composed with the representation $U_{\chi}$ of $\tilde{G}$, the operator $\Sigma$ satisfies the following properties, whose proofs consist in direct computations:

Proposition 5.22. Let $(M, s, \omega)$ be the polarized symplectic symmetric space associated to a transvection quadruple $(\tilde{G}, \tilde{\sigma}, \xi, B)$ (cf. Lemmas 5.14 and 5.16). Assume that the modular function of $B$ coincides with the restriction to $B$ of the modular function of $\tilde{G}$. Then the map

$$
\tilde{G} \rightarrow \mathcal{U}_{s a}\left(\mathcal{H}_{\chi}\right), \quad g \mapsto U_{\chi}(g) \Sigma U_{\chi}(g)^{*},
$$

is constant on the left cosets of $\tilde{K}$ in $\tilde{G}$. The corresponding mapping:

$$
\Omega: M=\tilde{G} / \tilde{K} \rightarrow \mathcal{U}_{s a}\left(\mathcal{H}_{\chi}\right), \quad g \tilde{K} \mapsto \Omega(g \tilde{K}):=U_{\chi}(g) \Sigma U_{\chi}(g)^{*},
$$

defines a unitary representation of the symmetric space $M=\tilde{G} / \tilde{K}$ in the sense that, for all $x, y$ in $M$ and $g$ in $\tilde{G}$, the following representative properties hold:

$$
\begin{aligned}
\Omega(x)^{2} & =\operatorname{Id}_{\mathcal{H}_{\chi}}, \\
\Omega(x) \Omega(y) \Omega(x) & =\Omega\left(s_{x} y\right), \\
U_{\chi}(g) \Omega(x) U_{\chi}(g)^{*} & =\Omega(g . x) .
\end{aligned}
$$

Definition 5.23. The pair $\left(\mathcal{H}_{\chi}, \Omega\right)$ is called the unitary representation of $(M, s)$ induced by the character $\chi$ of $B$.

We are now ready to define our prototype of quantization map on a polarized symplectic symmetric space:

Definition 5.24. Let $(M, s, \omega)$ be a the polarized symplectic symmetric space. Denote by $L^{1}(M)$ the space of integrable functions on $M$ with respect to the $\tilde{G}$ invariant (Liouville) measure $\mathrm{d}_{M}$. Denote by $\mathcal{B}\left(\mathcal{H}_{\chi}\right)$ the space of bounded linear operators on the Hilbert space $\mathcal{H}_{\chi}$. Consider the $\tilde{G}$-equivariant continuous linear map:

$$
\Omega: L^{1}(M) \rightarrow \mathcal{B}\left(\mathcal{H}_{\chi}\right), \quad f \mapsto \Omega(f):=\int_{M} f(x) \Omega(x) \mathrm{d}_{M}(x)
$$


The latter is called the quantization map of $M$ induced by the transvection quadruple $(\tilde{G}, \tilde{\sigma}, \xi, B)$.

REMARK 5.25. From $\|\Omega(x)\|=\|\Sigma\|=1$ (the norm here is the uniform norm on $\mathcal{B}\left(\mathcal{H}_{\chi}\right)$ ), we get the obvious estimate $\|\Omega(f)\| \leq\|f\|_{1}$, from which the continuity of the quantization map follows. Also, from Proposition 5.22 and from the $\tilde{G}$ invariance of $\mathrm{d}_{M}$, the covariance property at the level of the quantization map reads:

$$
U(g) \Omega(f) U(g)^{*}=\Omega\left({ }^{g} f\right), \quad \forall f \in L^{1}(M), \forall g \in \tilde{G},
$$

where ${ }^{g} f:=\left[g_{0} \tilde{K} \mapsto f\left(g^{-1} g_{0} \tilde{K}\right)\right]$. The latter equivariance property, under the full group of automorphisms of the symplectic symmetric space, is an important difference between the present "Weyl-type" construction and the classical coherentstate-quantization approach. For instance, as it appears already in the flat case of $\mathbb{R}^{2 n}$, holomorphic coherent-state-quantization (i.e. Berezin-Toeplitz in that case) yields a equivariance group that is isomorphic to $U(n) \ltimes \mathbb{C}^{n}$, while Weyl quantization is equivariant under the full automorphism group of $\mathbb{R}^{2 n}$ i.e. $\operatorname{Sp}(n, \mathbb{R}) \ltimes \mathbb{R}^{2 n}$. Another essential difference is unitarity (see Proposition 5.48 below). Lastly, the quantization defined above is in general not positive, i.e. for $0 \leq f \in L^{1}(M), \Omega(f)$ is not necessarily a positive operator. But since $\Omega(f)^{*}=\Omega(\bar{f})$, it maps real-valued functions to self-adjoint operators.

REMARK 5.26. The quantization map of Definition [5.24] is a generalization of the Weyl quantization, from the point of view of symmetric spaces. Moreover, we will see that for the symmetric space underlying a two-dimensional elementary normal $\mathbf{j}$-group (i.e. for the affine group of the real line) this construction coincides with Unterberger's Fuchs calculus [33.

Our next step is to introduce a functional parameter in the construction of the quantization map. There are several reasons for doing this, among which there is one of a purely analytical nature: obtaining a quantization map which is a unitary operator from the Hilbert space of square integrable symbols, $L^{2}(M)$, to the Hilbert space of Hilbert-Schmidt operators on $\mathcal{H}_{\chi}$, denoted by $\mathcal{L}^{2}\left(\mathcal{H}_{\chi}\right)$. This unitarity property will enable us to define a non-formal $\star$-product on $L^{2}(M)$ in a straightforward way.

Definition 5.27. Identifying a Borelian function $\mathbf{m}$ on $\tilde{G} / B$ with the operator on $\mathcal{H}_{\chi}$ of point-wise multiplication by this function, we let

$$
\Sigma_{\mathbf{m}}:=\mathbf{m} \circ \Sigma \text {. }
$$

When $\mathbf{m}$ is locally essentially bounded, the family of operators $U_{\chi}(g) \Sigma_{\mathbf{m}} U_{\chi}(g)^{*}$, $g \in \tilde{G}$, can be defined on the common domain $\Gamma_{c}^{\infty}\left(E_{\chi}\right)$. Note however that the latter family of operators is not necessarily constant on the left cosets of $\tilde{K}$ in $\tilde{G}$ and unless $\underline{\sigma}_{\tilde{K}}^{\star} \mathbf{m}=\mathbf{m}^{-1}$, one loses the involutive property for $\Sigma_{\mathbf{m}}$ (but one always keeps the $\tilde{G}$-equivariance). Also, these operators are bounded on $\mathcal{H}_{\chi}$, if and only if the function $\mathbf{m}$ is essentially bounded. But we will see in Theorem 5.43 that in order to obtain a unitary quantization map we are forced to consider such unbounded $\Sigma_{\mathbf{m}}$ 's. We mention a simple self-adjointness criterion, interesting on its own.

Lemma 5.28. Let $\mathbf{m}$ be a locally essentially bounded Borelian function on $\tilde{G} / B$ such that $\underline{\sigma}_{\tilde{K}}^{\star} \overline{\mathbf{m}}=\mathbf{m}$. Define

$$
\tilde{\Omega}_{\mathbf{m}}(g):=U_{\chi}(g) \Sigma_{\mathbf{m}} U_{\chi}(g)^{*}, \quad g \in \tilde{G},
$$


on the domain

$$
B_{g}:=\left\{\varphi \in \mathcal{H}_{\chi}:\left|\mathbf{m}_{g}\right| \varphi \in \mathcal{H}_{\chi}\right\} \quad \text { where } \quad \mathbf{m}_{g}:=\left[g_{0} B \mapsto \mathbf{m}\left(g^{-1} g_{0} B\right)\right], \quad g \in \tilde{G} .
$$

Then, $\tilde{\Omega}_{\mathbf{m}}(g)$ is self-adjoint on $\mathcal{H}_{\chi}$. Moreover, $\Gamma_{c}^{\infty}\left(E_{\chi}\right)$ is a common core for all $\tilde{\Omega}_{\mathbf{m}}(g)^{\prime} s, g \in \tilde{G}$.

PRoOF. Note first that the formal adjoint of $\Sigma_{\mathbf{m}}$ is $\Sigma_{\tilde{\sigma}_{\tilde{K}}^{\star}} \mathbf{m}$. Therefore, when $\underline{\sigma}_{\tilde{K}}^{\star} \overline{\mathbf{m}}=\mathbf{m}$, the operator $\Omega_{\mathbf{m}}(x)$ is symmetric on $\Gamma_{c}^{\infty}\left(E_{\chi}\right)$. Next, we remark that as both $\Sigma$ and $U_{\chi}(g), g \in \tilde{G}$, preserve $\Gamma_{c}^{\infty}\left(E_{\chi}\right)$, we get for $\varphi \in \Gamma_{c}^{\infty}\left(E_{\chi}\right)$ :

$$
\tilde{\Omega}_{\mathbf{m}}(g)^{2} \varphi=\left|\mathbf{m}_{g}\right|^{2} \varphi,
$$

that is, $\tilde{\Omega}_{\mathbf{m}}(g)$ squares on $\Gamma_{c}^{\infty}\left(E_{\chi}\right)$ to a multiplication operator. Since $\tilde{\Omega}_{\mathbf{m}}(g)$ is symmetric on the space of smooth compactly supported sections of $E_{\chi}$, the latter entails that

$$
\left\|\tilde{\Omega}_{\mathbf{m}}(g) \varphi\right\|=\left\|\left|\mathbf{m}_{g}\right| \varphi\right\|, \quad \forall \varphi \in \Gamma_{c}^{\infty}\left(E_{\chi}\right),
$$

and thus $\tilde{\Omega}_{\mathbf{m}}(g)$ is well defined on $B_{g}$. Then, the same computation as above, shows that $\tilde{\Omega}_{\mathbf{m}}(g)$ is also symmetric on its domain $B_{g}$. Observe that $B_{g}$ is complete in the graph norm, given by $\|\psi\|^{2}+\left\|\left|\mathbf{m}_{g}\right| \psi\right\|^{2}$, and that $\Gamma_{c}^{\infty}\left(E_{\chi}\right)$ is dense in $B_{g}$ for this norm. Thus $\tilde{\Omega}_{\mathbf{m}}(g)$, with domain $B_{g}$, is a closed operator. Clearly $B_{g} \subset$ $\operatorname{dom}\left(\tilde{\Omega}_{\mathbf{m}}(g)^{*}\right)$, since $\tilde{\Omega}_{\mathbf{m}}(g)$ is symmetric on $B_{g}$.

Choose an increasing sequence of relatively compact open sets $\left\{C_{n}\right\}_{n \in \mathbb{N}}$ in $\tilde{G} / B$, converging to $\tilde{G} / B$. For $n \in \mathbb{N}$, let $\chi_{n}$ be the indicator function of $C_{n}$. Then of course $\chi_{n} \varphi \in B_{g}$ for all $\varphi \in \mathcal{H}_{\chi}$. Note also that for $\varphi \in B_{g}$, we have by definition of $\mathbf{m}_{g}$ and from the relation $\underline{\sigma}_{\tilde{K}}^{\star} \mathbf{m}=\overline{\mathbf{m}}$ :

$$
\tilde{\Omega}_{\mathbf{m}}(g) \varphi=\mathbf{m}_{g} \Omega(x) \varphi=\Omega(x) \overline{\mathbf{m}}_{g} \varphi, \quad x=g \tilde{K} \in M .
$$

Thus for $g \in \tilde{G}, x=g \tilde{K} \in M, \psi \in \operatorname{dom}\left(\tilde{\Omega}_{\mathbf{m}}(g)^{*}\right), \varphi \in \mathcal{H}_{\chi}$ and using the fact that $\chi_{n} \mathbf{m}_{g}$ is essentially bounded (i.e. the associated multiplication operator is bounded), we get

$$
\left\langle\varphi, \chi_{n} \tilde{\Omega}_{\mathbf{m}}(g)^{*} \psi\right\rangle=\left\langle\tilde{\Omega}_{\mathbf{m}}(g) \chi_{n} \varphi, \psi\right\rangle=\left\langle\Omega(x) \overline{\mathbf{m}}_{g} \chi_{n} \varphi, \psi\right\rangle=\left\langle\varphi, \chi_{n} \mathbf{m}_{g} \Omega(x) \psi\right\rangle .
$$

Using the monotone convergence theorem, we obtain

$$
\begin{aligned}
\left\|\tilde{\Omega}_{\mathbf{m}}(g)^{*} \psi\right\| & =\lim _{n \rightarrow \infty}\left\|\chi_{n} \tilde{\Omega}_{\mathbf{m}}(g)^{*} \psi\right\|=\lim _{n \rightarrow \infty} \sup _{\|\varphi\|=1}\left|\left\langle\varphi, \chi_{n} \tilde{\Omega}_{\mathbf{m}}(g)^{*} \psi\right\rangle\right| \\
& =\lim _{n \rightarrow \infty} \sup _{\|\varphi\|=1}\left|\left\langle\varphi, \chi_{n} \mathbf{m}_{g} \Omega(x) \psi\right\rangle\right|=\lim _{n \rightarrow \infty}\left\|\chi_{n} \mathbf{m}_{g} \Omega(x) \psi\right\| \\
& =\left\|\mathbf{m}_{g} \Omega(x) \psi\right\|=\left\|\Omega(x) \overline{\mathbf{m}}_{g} \psi\right\|=\left\|\overline{\mathbf{m}}_{g} \psi\right\|=\left\|\left|\mathbf{m}_{g}\right| \psi\right\|,
\end{aligned}
$$

so that necessarily $\psi \in B_{g}$. Thus $\operatorname{dom}\left(\tilde{\Omega}_{\mathbf{m}}(g)^{*}\right)=B_{g}$, as required. Note lastly that $\Gamma_{c}^{\infty}\left(E_{\chi}\right)$ being dense in each $B_{g}$ for the graph norm, it is a common core for all the $\tilde{\Omega}_{\mathbf{m}}(g)$, which are therefore essentially selfadjoint on that domain.

REMARK 5.29. At this early stage of the construction, it is important to observe that our representation of $M$ (Proposition 5.22) and the associated quantization map (Definition 5.24) could have been equally defined starting with the full polarization quadruple $\left(\mathbb{L}, \sigma_{\mathbb{L}}, \xi, \mathbf{B}\right)$ (see Definition [5.19) of a non-exact polarized transvection triple $(\mathfrak{g}, \sigma, \varpi)$, instead of the transvection quadruple $\left(\tilde{G}, \tilde{\sigma},\left.\xi\right|_{\tilde{G}}, B\right)$. In particular, all the results of sections 5.3, 5.4, 5.5 and 5.6 can be thought as arising from the full quadruple. We will make great use of this observation in section 5.7 . 


\subsection{Locality and the one-point phase}

We next pass to the notion of locality in the context of transvection quadruples, out of which we will be able to give an explicit expression of the operators $\Omega_{\mathbf{m}}(x)$ on $\mathcal{H}_{\chi}$.

Definition 5.30. Within the notations of Definition 5.19, we say that the polarized symplectic symmetric space $(M, s, \omega)$, associated to a non-exact polarized transvection triple $(\mathfrak{g}, \sigma, \varpi)$, is local whenever there exists a subgroup $Q$ of $\tilde{G}$ such that:

(i) The map

$$
Q \times B \rightarrow \tilde{G}, \quad(q, b) \mapsto q b .
$$

is a global diffeomorphism. In particular, $Q$ is closed as a subgroup of $\tilde{G}$.

(ii) For all $q \in Q$ and $b \in B$, one has

$$
\mathbf{C}_{q}\left(\tilde{\sigma}(b) b^{-1}\right) \in B
$$

where $\mathbf{C}_{g}\left(g^{\prime}\right):=g g^{\prime} g^{-1}$ denotes the conjugate action of $\tilde{G}$ on itself.

(iii) For every $q \in Q$, setting $\tilde{\sigma} q=:(\tilde{\sigma} q)^{Q}(\tilde{\sigma} q)^{B}$ relatively to the global decomposition $\tilde{G}=Q . B$, one has:

$$
\chi\left((\tilde{\sigma} q)^{B}\right)=1 .
$$

For a local symplectic symmetric space, the identification $Q \simeq \tilde{G} / B$ allows to transfer the symmetric space structure of the former to the latter:

Lemma 5.31. Let $(M, s, \omega)$ be a local symplectic symmetric space. Then:

(i) The mapping:

$$
\underline{s}: Q \times Q \rightarrow Q, \quad\left(q, q^{\prime}\right) \mapsto \underline{s}_{q}\left(q^{\prime}\right):=q\left(\tilde{\sigma}\left(q^{-1} q^{\prime}\right)\right)^{Q},
$$

defines a left-invariant structure of symmetric space on the Lie group $Q$.

(ii) Moreover, the global diffeomorphism

$$
Q \rightarrow \tilde{G} / B, \quad q \mapsto q B,
$$

intertwines the symmetry $\underline{s}$ with the involution $\underline{\sigma}$, defined in (5.6) for the transvection quadruple $(\tilde{G}, \tilde{\sigma}, \xi, B)$ :

$$
\underline{s}_{q} q^{\prime} \mapsto \underline{\sigma}_{q \tilde{K}}\left(q^{\prime} B\right), \quad \forall q, q^{\prime} \in Q .
$$

(iii) Under the identification $Q \simeq \tilde{G} / B$ given above, the $\left(\tilde{G}, \underline{\sigma}_{\tilde{K}}\right)$-invariant measure $\mathrm{d}_{\tilde{G} / B}$ on $\tilde{G} / B$ constructed in Lemma 5.17, becomes a $\left(\tilde{G}, \underline{s}_{e}\right)$ invariant measure $\mathrm{d}_{Q}$ on the Lie group $Q$, which is also a left-invariant Haar measure on $Q$.

(iv) Last, we have an isomorphism of Hilbert spaces $\mathcal{H}_{\chi} \simeq L^{2}(Q)$ induced by the $\tilde{G}$-equivariant isomorphism:

$$
C^{\infty}(\tilde{G})^{B} \rightarrow C^{\infty}(Q), \quad \hat{\varphi} \mapsto \varphi:=\left.\hat{\varphi}\right|_{Q},
$$

under which, we have $\Sigma=\underline{s}_{e}^{\star}$.

Proof. Item (ii) follows from a direct check implying in turn the left- $Q$ equivariance of $\underline{s}$ from the left- $\tilde{G}$-equivariance of $\underline{\sigma}$. The fact that $\underline{s}_{q}$ fixes $q$ isolatedly is a consequence of the following observation. Considering the linear epimorphism 
$p_{\star \tilde{K}}: \mathfrak{p} \rightarrow \mathfrak{q}=T_{e} Q \simeq T_{B}(\tilde{G} / B)$ tangent to the projection $p: M \simeq \tilde{G} / \tilde{K} \rightarrow \tilde{G} / B \simeq$ $Q, g \tilde{K} \mapsto g B$, one observes that for every $X \in \mathfrak{p}$ :

$$
\underline{\sigma}_{\tilde{K} \star B}\left(p_{\star \tilde{K}} X\right)=\left(p \circ s_{\tilde{K}}\right)_{\star \tilde{K}}(X)=-p_{\star \tilde{K}} X .
$$

Hence $\underline{\sigma}_{\tilde{K} \star B}=-i d_{\mathfrak{q}}$ and (i) follows. Last, (iii) and (iv) are immediate consequences of the $Q$-equivariant identification $Q \simeq \tilde{G} / B$.

From now on, we will always make the identification $\mathcal{H}_{\chi} \simeq L^{2}(Q)$, under which we can derive the action of the individual operators $\Omega(x), x \in M$. For this, we need a preliminary result:

Lemma 5.32. Let $(M, s, \omega)$ be a local symplectic symmetric space and let $\hat{\varphi} \in$ $C^{\infty}(\tilde{G}, \mathbb{C})^{B}$ be a B-equivariant function. Then, for all $q, q_{0} \in Q$ and $b \in B$, one has:

with

$$
L_{q b}^{\star} \circ \tilde{\sigma}^{\star} \circ L_{(q b)^{-1}}^{\star} \hat{\varphi}\left(q_{0}\right)=\mathbf{E}\left(q_{0}^{-1} q b\right) \hat{\varphi}\left(\underline{s}_{q} q_{0}\right)
$$

Proof. A direct computation yields:

$L_{q b}^{\star} \circ \tilde{\sigma}^{\star} \circ L_{(q b)^{-1}}^{\star} \hat{\varphi}\left(q_{0}\right)=\hat{\varphi}\left(q b \tilde{\sigma}\left(b^{-1}\right) \tilde{\sigma}\left(q^{-1} q_{0}\right)\right)=\hat{\varphi}\left(q \tilde{\sigma}\left(q^{-1} q_{0}\right) \mathbf{C}_{\tilde{\sigma}\left(q_{0}^{-1} q\right)}\left(b \tilde{\sigma}\left(b^{-1}\right)\right)\right)$.

Under the assumption of locality (Definition 5.30), we have $\mathbf{C}_{\tilde{\sigma}\left(q_{0}^{-1} q\right)}\left(b \tilde{\sigma}\left(b^{-1}\right)\right) \in B$. The $\tilde{\sigma}$-invariance of $\chi$ and item (iii) of Definition 5.30 then yield the formula.

REMARK 5.33. We call the function $\mathbf{E}$ in (5.11) the one-point phase. Observe that the latter is well defined thanks to the second condition in the assumption of locality (Definition 5.30).

Corollary 5.34. Let $(M, s, \omega)$ be a local symplectic symmetric space. For $\varphi \in L^{2}(Q)$ and $x=q b \tilde{K} \in M, q \in Q, b \in B$, we have

$$
\Omega(x) \varphi\left(q_{0}\right)=\mathbf{E}\left(q_{0}^{-1} q b\right) \varphi\left(\underline{s}_{q}\left(q_{0}\right)\right),
$$

where $\mathbf{E}$ is the phase defined in (5.11) and $\underline{s}$ is the symmetry of the Lie group $Q$ constructed in Lemma 5.31.

\subsection{Unitarity and midpoints for elementary spaces}

In addition to locality (Definition 5.30), we will assume further conditions on the structure of our polarized symplectic symmetric space $(M, s, \omega)$, which will enable us to give an explicit expression of the three-point kernel associated to a WKB-quantization of $M$ as well as to prove the triviality of the associated Berezin transform (see Definition 5.46 below). Recall that the notion of midpoint map on a symmetric space is given in Definition 2.10 .

Definition 5.35. A local symplectic symmetric space $(M, s, \omega)$ is called elementary when, within the context of the section 5.2, the following additional conditions are satisfied:

(i) The symmetric space $(Q, \underline{s})$ is solvable and admits a (necessary unique) midpoint map. (By a result proved in [34, this implies that $Q$ is exponential.) 
(ii) There exists an exponential Lie subgroup $\mathbb{Y}$ of $B$ normalized by $Q$ and such that the semi-direct product

$$
\mathbb{S}:=Q \ltimes \mathbb{Y} \subset \tilde{G},
$$

acts simply transitively on $M$.

(iii) Denoting by $\mathfrak{Y}$ the Lie algebra of $\mathbb{Y}$, there exists a global diffeomorphism $\Psi: Q \rightarrow \mathfrak{q}$ such that

$$
\left\langle\xi,\left(\operatorname{Ad}_{q^{-1}}-\operatorname{Ad}_{\left(\underline{s}_{e} q\right)^{-1}}\right) y\right\rangle=\langle\xi,[\Psi(q), y]\rangle, \quad \forall y \in \mathfrak{Y}, \forall q \in Q .
$$

(iv) The Lie algebras $\mathfrak{Y}$ and $\mathfrak{q}$ are Lagrangian subspaces of the Lie algebra $\mathfrak{s}$ of $\mathbb{S}$ that are in symplectic duality with respect to the evaluation at the unit element $e$ of $\mathbb{S}$ of the symplectic structure transported from $M$ to $\mathbb{S}$ via the diffeomorphism $\mathbb{S} \rightarrow M=\tilde{G} / \tilde{K}, x \mapsto x \tilde{K}$.

REMARK 5.36. Let $(M, s, \omega)$ be an elementary symplectic symmetric space.

(i) From now on, we always make the $\mathbb{S}$-equivariant identification:

$$
\mathbb{S}=Q \ltimes \mathbb{Y} \rightarrow M, \quad q b \mapsto q b \tilde{K} .
$$

Observe that under this identification, the $\tilde{G}$-invariant Liouville measure $\mathrm{d}_{M}$ on $M$ is a left Haar measure on $\mathbb{S}$, which under the parametrization $g=q b, q \in Q, b \in \mathbb{Y}$, is proportional to any product of left invariant Haar measures on $Q$ and on $\mathbb{Y}$. We simply denote the latter by $\mathrm{d}_{\mathbb{S}}$.

(ii) For $\mathbf{m}$ a locally essentially bounded Borelian function on $Q$ and $x \in \mathbb{S}$, the operator $\tilde{\Omega}_{\mathbf{m}}(x)$ given in Lemma 5.28 will be simply denoted by $\Omega_{\mathbf{m}}(x)$. This is coherent with the identification above and with the notation of Proposition 5.22 when $\mathbf{m}=1$. Moreover, the family $\left\{\Omega_{\mathbf{m}}(x)\right\}_{x \in \mathbb{S}}$ satisfies the first axiom, (5.1), of a covariant Moyal quantizer, as given at the very beginning of this chapter.

(iii) Since $Q$ normalizes $\mathbb{Y}$, the restriction to $\mathbb{S}=Q \ltimes \mathbb{Y}$ of the representation $U_{\chi}$ of $\tilde{G}$ on $\mathcal{H}_{\chi} \simeq L^{2}(Q)$ given in (5.9), reads:

$$
U_{\chi}(q b) \varphi\left(q_{0}\right)=\chi\left(\mathbf{C}_{q_{0}^{-1} q}(b)\right) \varphi\left(q^{-1} q_{0}\right) .
$$

In the elementary case, we observe the following relation between the one-point phase $\mathbf{E}$ and the diffeomorphism $\Psi: Q \rightarrow \mathfrak{q}$ :

Lemma 5.37. Let $(M, s, \omega)$ be an elementary symplectic symmetric space. Then, for $q \in Q$ and $y \in \mathfrak{Y}$, we have:

$$
\mathbf{E}\left(q^{-1} e^{y}\right)=\exp \{i\langle\xi,[\Psi(q), y]\rangle\} .
$$

Proof. By definition, we have for $q \in Q$ and $b \in \mathbb{Y}$ :

$$
\mathbf{E}\left(q^{-1} b\right)=\bar{\chi}\left(\tilde{\sigma}\left(\mathbf{C}_{\tilde{\sigma}\left(q^{-1}\right)}(b)\right) \mathbf{C}_{q^{-1}}\left(b^{-1}\right)\right) .
$$

Next, we write

$$
\tilde{\sigma}\left(q^{-1}\right)=\tilde{\sigma}(q)^{-1}=\left(\tilde{\sigma}(q)^{Q} \tilde{\sigma}(q)^{B}\right)^{-1}=\left(\tilde{\sigma}(q)^{B}\right)^{-1}\left(\underline{s}_{e} q\right)^{-1},
$$

to get

$$
\mathbf{C}_{q^{-1}}\left(b^{-1}\right) \tilde{\sigma}\left(\mathbf{C}_{\tilde{\sigma}\left(q^{-1}\right)}(b)\right)=\left(\tilde{\sigma}\left(\tilde{\sigma}(q)^{B}\right)^{-1}\right) \tilde{\sigma}\left(\mathbf{C}_{\left(\underline{s}_{e} q\right)^{-1}}(b)\right) \tilde{\sigma}\left(\tilde{\sigma}(q)^{B}\right)\left(\mathbf{C}_{q^{-1}}\left(b^{-1}\right)\right) .
$$


Since $Q$ normalizes $\mathbb{Y}$ and $B$ is $\tilde{\sigma}$-stable, we observe that each of the four factors in the right hand side above, belong to $B$. Thus, we can split $\mathbf{E}\left(q^{-1} b\right)$ according to this decomposition, to get

$$
\mathbf{E}\left(q^{-1} b\right)=\chi\left(\mathbf{C}_{q^{-1}}(b)\right) \bar{\chi}\left(\mathbf{C}_{\left(\underline{s}_{e} q\right)^{-1}}(b)\right) .
$$

The result follows from the definition of the diffeomorphism $\Psi$ and the character $\chi$.

Now, using Corollary 5.34, Definition 5.27 and Lemma 5.28, under the identification $\mathbb{S} \simeq M$, we note

Proposition 5.38. Let $(M, s, \omega)$ be an elementary symplectic symmetric space. Let $q \in Q, b \in \mathbb{Y}$ and let $\mathbf{m}$ be an essentially locally bounded Borelian function on $Q$. Then the densely defined (on $B_{q b}=B_{q}$-see Definition 5.27 and Lemma 5.28) operator $\Omega_{\mathbf{m}}(q b)$, acts as:

$$
\Omega_{\mathbf{m}}(q b) \varphi\left(q_{0}\right)=\mathbf{m}\left(q^{-1} q_{0}\right) \mathbf{E}\left(q_{0}^{-1} q b\right) \varphi\left(\underline{s}_{q} q_{0}\right), \quad \forall \varphi \in B_{q} \subset \mathcal{H}_{\chi}, \quad \forall q_{0} \in Q .
$$

Corollary 5.39. Let $(M, s, \omega)$ be an elementary symplectic symmetric space, $\mathbf{m}$ be an essentially locally bounded Borelian function on $Q$ and $f \in \mathcal{D}(\mathbb{S})$. Then the operator $\Omega_{\mathbf{m}}(f)$ defined by

$$
\begin{aligned}
\Omega_{\mathbf{m}}(f): \mathcal{D}(Q) & \rightarrow \mathcal{D}^{\prime}(Q), \\
\varphi \mapsto \Omega_{\mathbf{m}}(f) \varphi:=[\psi \in \mathcal{D}(Q) & \left.\mapsto \int_{Q \times \mathbb{S}} \psi\left(q_{0}\right) f(q b)\left(\Omega_{\mathbf{m}}(q b) \varphi\right)\left(q_{0}\right) \mathrm{d}_{\mathbb{S}}(q b) \mathrm{d}_{Q}\left(q_{0}\right)\right],
\end{aligned}
$$

has a distributional kernel given by

$$
\begin{aligned}
& \Omega_{\mathbf{m}}(f)\left[q_{0}, q\right]= \\
& \mathbf{m}\left(\operatorname{mid}\left(e, q_{0}^{-1} q\right)^{-1}\right)\left|\mathrm{Jac}_{\left(\underline{s}^{e}\right)^{-1}}\right|\left(q_{0}^{-1} q\right) \int_{\mathbb{Y}} f\left(\operatorname{mid}\left(q_{0}, q\right) b\right) \mathbf{E}\left(\operatorname{mid}\left(e, q_{0}^{-1} q\right) b\right) \mathrm{d}_{\mathbb{Y}}(b) .
\end{aligned}
$$

Proof. Observe first that under the decomposition $\mathbb{S}=Q \ltimes \mathbb{Y}$, the left Haar measure on $\mathbb{S}$ coincides with the product of left Haar measures on $Q$ and $\mathbb{Y}$ :

$$
\mathrm{d}_{\mathbb{S}}(q b)=\mathrm{d}_{\mathbf{Q}}(q) \mathrm{d}_{\mathbb{Y}}(b), \quad \forall q \in Q, \forall b \in \mathbb{Y} .
$$

For $f \in \mathcal{D}(\mathbb{S})$ and any Borelian $\mathbf{m}$, it is clear that $\Omega_{\mathbf{m}}(f)$ defines a continuous operator from $\mathcal{D}(Q)$ to $\mathcal{D}^{\prime}(Q)$ and acts as:

$\Omega_{\mathbf{m}}(f) \varphi\left(q_{0}\right)=\int_{Q \ltimes \mathbb{Y}} f(q b) \mathbf{m}\left(q^{-1} q_{0}\right) \mathbf{E}\left(q_{0}^{-1} q b\right) \varphi\left(\underline{s}_{q}\left(q_{0}\right)\right) \mathrm{d}_{Q}(q) \mathrm{d}_{\mathbb{Y}}(b), \quad \varphi \in \mathcal{D}(Q)$.

For any $q_{0} \in Q$, we set $q^{\prime}(q):=\underline{s}_{q}\left(q_{0}\right)$ and we get from the defining property of the midpoint map that $q=\operatorname{mid}\left(q_{0}, q^{\prime}\right)$. Now observe that left-translations (in the group $Q)$ are automorphisms of the symmetric space $(Q, \underline{s})$. Indeed, for all $q_{0}, q, q^{\prime}$ in $Q$, we have

$$
L_{q_{0}}\left(\underline{s}_{q}\left(L_{q_{0}^{-1}} q^{\prime}\right)\right)=q_{0} q\left(\tilde{\sigma}\left(q^{-1} q_{0}^{-1} q^{\prime}\right)\right)^{Q}=\underline{s}_{q_{0} q}\left(q^{\prime}\right) .
$$

Hence, by Remark 2.12, we get

$$
\operatorname{mid}\left(q_{0}, q^{\prime}\right)=q_{0} \operatorname{mid}\left(e, q_{0}^{-1} q^{\prime}\right)=L_{q_{0}} \circ\left(\underline{s}^{e}\right)^{-1} \circ L_{q_{0}^{-1}}\left(q^{\prime}\right),
$$

the invariance of the Haar measure $\mathrm{d}_{Q}$ under left translation gives:

$$
\left|\operatorname{Jac}_{\operatorname{mid}\left(q_{0}, .\right)}\right|\left(q^{\prime}\right)=\left|\operatorname{Jac}_{\left(\underline{s}^{e}\right)^{-1}}\right|\left(q_{0}^{-1} q^{\prime}\right) \text {. }
$$


Therefore, a direct computation shows that:

$$
\begin{aligned}
& \Omega_{\mathbf{m}}(f) \varphi\left(q_{0}\right)=\int_{Q \ltimes \mathbb{Y}} f\left(\operatorname{mid}\left(q_{0}, q\right) b\right) \mathbf{m}\left(\operatorname{mid}\left(e, q_{0}^{-1} q\right)^{-1}\right)\left|\mathrm{Jac}_{\left(\underline{s}^{e}\right)^{-1}}\right|\left(q_{0}^{-1} q\right) \\
& \times \mathbf{E}\left(\operatorname{mid}\left(e, q_{0}^{-1} q\right) b\right) \varphi(q) \mathrm{d}_{Q}(q) \mathrm{d}_{\mathbb{Y}}(b),
\end{aligned}
$$

and the result follows by identification.

REMARK 5.40. As a consequence of the preceding corollary, we deduce that for an elementary symplectic symmetric space, the second axiom, (5.2), of a covariant Moyal quantizer is satisfied when $\mathbf{m}(e)\left|\operatorname{Jac}_{\left(\underline{s}^{e}\right)^{-1}}\right|(e)=1$. Indeed, for $f \in \mathcal{D}(\mathbb{S})$, we deduce that

$$
\begin{aligned}
\operatorname{Tr}\left[\Omega_{\mathbf{m}}(f)\right] & =\int_{Q} \Omega_{\mathbf{m}}(f)[q, q] \mathrm{d}_{Q}(q) \\
& =\mathbf{m}(e)\left|\operatorname{Jac}_{\left(\underline{s}^{e}\right)^{-1}}\right|(e) \int_{Q \times \mathbb{Y}} f(q b) \mathbf{E}(b) \mathrm{d}_{\mathbb{Y}}(b) \mathrm{d}_{Q}(q)=\int_{\mathbb{S}} f(x) \mathrm{d}_{\mathbb{S}}(x),
\end{aligned}
$$

since $\mathbf{E}(b)=1$ for all $\mathfrak{b} \in \mathbb{Y}$ by Definition 5.35 (iii) and Lemma 5.37

Our next aim is to understand the geometrical conditions on the functional parameter $\mathbf{m}$ necessary for the quantization map to extend to a unitary operator from $L^{2}(M)$ to the Hilbert space of Hilbert-Schmidt operators on $\mathcal{H}_{\chi}$. For this, we introduce the following specific function on $Q$ :

Definition 5.41. For $(M, s, \omega)$ an elementary symplectic symmetric space, define the function $\mathbf{m}_{0}$ on $Q$ as:

$$
\mathbf{m}_{\mathbf{0}}(q):=\left|\operatorname{Jac}_{\underline{s}^{e}}\left(q^{-1}\right) \operatorname{Jac}_{\Psi}(q)\right|^{1 / 2} .
$$

REMARK 5.42. Observe that both $\left|\mathrm{Jac}_{\Psi}\right|$ and $\left|\mathrm{Jac}_{\underline{s}}\right|$ are $\underline{s}_{e}^{\star}$-invariant. Indeed, we have $\Psi \circ \underline{s}_{e}=-\Psi$ and $\underline{s}^{e} \circ \underline{s}_{e}=\underline{s}_{e} \circ \underline{s}^{e}$, hence the claim follows from $\left|J \mathrm{Jac}_{\underline{s}_{e}}\right|=1$ (cf. Lemma $5.31(\mathrm{iii})$ ). However, $\mathbf{m}_{0}$ need not be $\underline{s}_{e}^{\star}$-invariant, as $\left|\mathrm{Jac}_{\underline{\underline{s}}}\right|$ need not be invariant under the inversion map on $Q$. Thus, from Lemma 5.28, the quantization map $\Omega_{\mathbf{m}_{0}}$, need not send real functions to self-adjoint operators.

We can now state one of the main results of this chapter:

THEOREM 5.43. Let $(M, s, \omega)$ be an elementary symplectic symmetric space and $\mathbf{m}$ be a Borelian function on $Q$ which is (almost everywhere) dominated by $\mathbf{m}_{\mathbf{0}}$ and assume that $\mathbb{Y}$ is Abelian. Then the quantization map:

$$
\Omega_{\mathbf{m}}: f \mapsto \Omega_{\mathbf{m}}(f):=\int_{\mathbb{S}} f(q b) \Omega_{\mathbf{m}}(q b) \mathrm{d}_{\mathbb{S}}(q b),
$$

is a bounded operator from $L^{2}(\mathbb{S})$ to $\mathcal{L}^{2}\left(\mathcal{H}_{\chi}\right)$ with

$$
\left\|\Omega_{\mathbf{m}}\right\| \leq\left\|\mathbf{m} / \mathbf{m}_{0}\right\|_{\infty} .
$$

Moreover, $\Omega_{\mathbf{m}}$ is a unitary operator if and only if $|\mathbf{m}|=\mathbf{m}_{\mathbf{0}}$.

Proof. Recall that a linear operator $T: \mathcal{D}(Q) \rightarrow \mathcal{D}^{\prime}(Q)$ extends to a HilbertSchmidt operator on $L^{2}(Q)$, if and only its distributional kernel belongs to $L^{2}(Q \times$ $Q)$. In this case, its Hilbert-Schmidt norm coincides with the $L^{2}$-norm of its kernel. 
Thus by Corollary 5.39, we deduce that if $f \in \mathcal{D}(\mathbb{S})$, then the square of the HilbertSchmidt norm of $\Omega_{\mathrm{m}}(f)$ reads

$$
\begin{aligned}
& \left\|\Omega_{\mathbf{m}}(f)\right\|_{2}^{2}= \\
& \int_{Q^{2} \times \mathbb{Y}^{2}}|\mathbf{m}|^{2}\left(\operatorname{mid}\left(e, q_{0}^{-1} q\right)^{-1}\right)\left|\operatorname{Jac}_{\left(\underline{s}^{e}\right)^{-1}}\right|^{2}\left(q_{0}^{-1} q\right) \bar{f}\left(\operatorname{mid}\left(q_{0}, q\right) b\right) f\left(\operatorname{mid}\left(q_{0}, q\right) b_{0}\right) \\
& \times \\
& \times \overline{\mathbf{E}}\left(\operatorname{mid}\left(e, q_{0}^{-1} q\right) b\right) \mathbf{E}\left(\operatorname{mid}\left(e, q_{0}^{-1} q\right) b_{0}\right) \mathrm{d}_{Q}(q) \mathrm{d}_{\mathbb{Y}}(b) \mathrm{d}_{Q}\left(q_{0}\right) \mathrm{d}_{\mathbb{Y}}\left(b_{0}\right) .
\end{aligned}
$$

Performing the change of variables $\operatorname{mid}\left(q_{0}, q\right) \mapsto q$ (the inverse of the one we performed in the proof of Corollary 5.39) and using the relation between the function $\mathbf{E}$ and the diffeomorphism $\Psi: Q \rightarrow q$ given in Lemma [5.37, we get the following expression for $\left\|\Omega_{\mathbf{m}}(f)\right\|_{2}^{2}$ :

$$
\begin{aligned}
\int_{Q^{2} \times \mathbb{Y}^{2}}|\mathbf{m}|^{2}\left(q^{-1} q_{0}\right)\left|\operatorname{Jac}_{\left(\underline{s}^{e}\right)^{-1}}\right|\left(\underline{s}^{e}\left(q_{0}^{-1} q\right)\right) \bar{f}(q b) & f\left(q b_{0}\right) e^{i\left\langle\xi,\left[\Psi\left(q^{-1} q_{0}\right), \log (b)-\log \left(b_{0}\right)\right]\right\rangle} \\
& \times \mathrm{d}_{Q}(q) \mathrm{d}_{\mathbb{Y}}(b) \mathrm{d}_{Q}\left(q_{0}\right) \mathrm{d}_{\mathbb{Y}}\left(b_{0}\right)
\end{aligned}
$$

and the latter can be rewritten as

$$
\int_{Q^{2} \times \mathbb{Y}^{2}} \frac{|\mathbf{m}|^{2}\left(q_{0}\right)}{\left|\operatorname{Jac}_{\underline{s}}\right|\left(q_{0}^{-1}\right)} \bar{f}(q b) f\left(q b_{0}\right) e^{i\left\langle\xi,\left[\Psi\left(q_{0}\right), \log (b)-\log \left(b_{0}\right)\right]\right\rangle} \mathrm{d}_{Q}(q) \mathrm{d}_{\mathbb{Y}}(b) \mathrm{d}_{Q}\left(q_{0}\right) \mathrm{d}_{\mathbb{Y}}\left(b_{0}\right),
$$

where in the last line, we used left-invariance of the Haar measure on $Q$. Setting $w_{0}=\Psi\left(q_{0}\right) \in \mathfrak{q}$ and $\mathrm{d} w_{0}$ the Lebesgue measure on $\mathfrak{q}$, we get

$$
\begin{aligned}
\left\|\Omega_{\mathbf{m}}(f)\right\|_{2}^{2} & =\int_{Q \times \mathfrak{q} \times \mathbb{Y}^{2}} \frac{|\mathbf{m}|^{2}\left(\Psi^{-1}\left(w_{0}\right)\right) \bar{f}(q b) f\left(q b_{0}\right)}{\left|\operatorname{Jac}_{\underline{s}^{e}}\right|\left(\Psi^{-1}\left(w_{0}\right)^{-1}\right)\left|\operatorname{Jac}_{\Psi}\right|\left(\Psi^{-1}\left(w_{0}\right)\right)} e^{i\left\langle\xi,\left[w_{0}, \log (b)-\log \left(b_{0}\right)\right]\right\rangle} \\
\times \mathrm{d}_{Q}(q) \mathrm{d}_{\mathbb{Y}}(b) \mathrm{d} w_{0} \mathrm{~d}_{\mathbb{Y}}\left(b_{0}\right) & \quad \times \mathrm{d}_{Q}(q) \mathrm{d}_{\mathbb{Y}}(b) \mathrm{d} w_{0} \mathrm{~d}_{\mathbb{Y}}\left(b_{0}\right) \\
= & \int_{Q \times \mathfrak{q} \times \mathbb{Y}^{2}} \frac{|\mathbf{m}|^{2}\left(\Psi^{-1}\left(w_{0}\right)\right)}{\mathbf{m}_{\mathbf{0}^{2}\left(\Psi^{-1}\left(w_{0}\right)\right)}} \bar{f}(q b) f\left(q b_{0}\right) e^{-i\left\langle\xi,\left[w_{0}, \log (b)-\log \left(b_{0}\right)\right]\right\rangle} \\
& =\int_{Q \times \mathfrak{q}} \frac{|\mathbf{m}|^{2}\left(\Psi^{-1}\left(w_{0}\right)\right)}{\mathbf{m}_{\mathbf{0}^{2}}^{2}\left(\Psi^{-1}\left(w_{0}\right)\right)}\left|\int_{\mathbb{Y}} f(q b) e^{-i\left\langle\xi,\left[w_{0}, \log (b)\right]\right\rangle} \mathrm{d}_{\mathbb{Y}}(b)\right|^{2} \mathrm{~d}_{Q}(q) \mathrm{d} w_{0} .
\end{aligned}
$$

We will next use the relation (which follows from the construction of $\xi \in \tilde{\mathfrak{g}}^{\star}$ ):

$$
\langle\xi,[X, Y]\rangle=\varpi(X, Y), \quad \forall X, Y \in \tilde{\mathfrak{g}},
$$

and the fact that $\mathfrak{q}$ and $\mathfrak{Y}$ are Lagrangian subspaces in symplectic duality (see Definition (5.35). Now, since $\mathbb{Y}$ is Abelian and exponential, the exponential map $\mathfrak{Y} \rightarrow \mathbb{Y}: y \mapsto \exp (y)$ is a measure preserving diffeomorphism (the vector space $\mathfrak{Y}$ being endowed with a normalized Lebesgue measure $\mathrm{d} y$ ) inducing the isometric linear identification:

$$
\exp ^{\star}: L^{2}(\mathbb{Y}) \rightarrow L^{2}(\mathfrak{Y})
$$

Within this set-up, the (isometrical) Fourier transform reads:

$$
\mathcal{F}: L^{2}(\mathbb{Y}) \rightarrow L^{2}(\mathfrak{q}), \quad \varphi \mapsto\left[w_{0} \mapsto \int_{\mathbb{Y}} \varphi(b) e^{-i\left\langle\xi,\left[w_{0}, \log (b)\right]\right\rangle} \mathrm{d}_{\mathbb{Y}}(b)\right],
$$

where, again, the vector space $\mathfrak{q}$ is endowed with a normalized Lebesgue measure $\mathrm{d} w_{0}$. We therefore observe that

$$
\left\|\Omega_{\mathbf{m}}(f)\right\|_{2}^{2}=\int_{Q \times \mathfrak{q}} \frac{|\mathbf{m}|^{2}\left(\Psi^{-1}\left(w_{0}\right)\right)}{\mathbf{m}_{\mathbf{0}}^{2}\left(\Psi^{-1}\left(w_{0}\right)\right)}\left|\mathcal{F}\left(L_{q}^{\star} f\right)\left(w_{0}\right)\right|^{2} \mathrm{~d}_{Q}(q) \mathrm{d} w_{0},
$$


where we set $L_{q}^{\star} f: b \mapsto f(q b)$. Hence, we see that if $|\mathbf{m}| \leq C \mathbf{m}_{\mathbf{0}}$, we have:

$$
\begin{aligned}
\left\|\Omega_{\mathbf{m}}(f)\right\|_{2}^{2} \leq & C^{2} \int_{Q \times \mathfrak{q}}\left|\mathcal{F}\left(L_{q}^{\star} f\right)\left(w_{0}\right)\right|^{2} \mathrm{~d} w_{0} \mathrm{~d}_{Q}(q) \\
& =C^{2} \int_{Q \times \mathbb{Y}}\left|L_{q}^{\star} f(b)\right|^{2} \mathrm{~d}_{\mathbb{Y}}(b) \mathrm{d}_{Q}(q) \\
& =C^{2}\|f\|_{2}^{2}, \quad \forall f \in \mathcal{D}(\mathbb{S}) .
\end{aligned}
$$

i.e.

$$
\left\|\Omega_{\mathbf{m}}(f)\right\|_{2} \leq C\|f\|_{2}, \quad \forall f \in \mathcal{D}(\mathbb{S}) .
$$

By density, we deduce that $\Omega_{\mathbf{m}}(f)$ is Hilbert-Schmidt for all $f \in L^{2}(\mathbb{S})$ and with equality of norms if and only if $|\mathbf{m}|=\mathbf{m}_{\mathbf{0}}$. In this case, a similar computation shows that for any $f_{1}, f_{2} \in L^{2}(\mathbb{S})$, we have

$$
\operatorname{Tr}\left[\Omega_{\mathbf{m}}\left(f_{1}\right)^{*} \Omega_{\mathbf{m}}\left(f_{2}\right)\right]=\int_{\mathbb{S}} \bar{f}_{1}(q b) f_{2}(q b) \mathrm{d}_{\mathbb{S}}(q b),
$$

which terminates the proof.

REMARK 5.44. Let $(M, s, \omega)$ be an elementary symplectic symmetric space and $\mathbf{m}$ be an essentially bounded function on $Q$. By Lemma 5.28, we know that when $\underline{s}_{e}^{\star} \overline{\mathbf{m}}=\mathbf{m}$, then the family $\left\{\Omega_{\mathbf{m}_{0}}(x)\right\}_{x \in \mathbb{S}}$ consists of self-adjoint operators. By Remarks 5.36 (ii) and 5.40 we also know (under a mild normalization condition) that the first two axioms, (5.1) and (5.2), of a covariant Moyal quantizer (as defined

at the beginning of this chapter) are satisfied. Now, observe that the content of the previous Lemma can be summarized as follow:

$$
\operatorname{Tr}\left[\Omega_{\underline{s}_{e}^{\star}} \overline{\mathbf{m}}(x) \Omega_{\mathbf{m}}(y)\right]=\delta_{x}(y) \Longleftrightarrow|\mathbf{m}|=\mathbf{m}_{\mathbf{0}} .
$$

Hence, when $\mathbf{m}_{0}$ is $\underline{s}_{e}$-invariant, then the family $\left\{\Omega_{\mathbf{m}_{0}}(x)\right\}_{x \in \mathbb{S}}$ satisfies also the third axiom (5.3).

\subsection{The $\star$-product as the composition law of symbols}

DeFinition 5.45 . Let $(M, s, \omega)$ be an elementary symplectic symmetric space such that $\mathbf{m} / \mathbf{m}_{0} \in L^{\infty}(Q)$ and such that $\mathbb{Y}$ is Abelian. Then let

$$
\sigma_{\mathbf{m}}: \mathcal{L}^{2}\left(\mathcal{H}_{\chi}\right) \rightarrow L^{2}(\mathbb{S})
$$

be the adjoint of the quantization map $\Omega_{\mathbf{m}}$. We call the latter the symbol map.

Recall that the defining property of the symbol map is

$$
\left\langle f, \sigma_{\mathbf{m}}[A]\right\rangle=\operatorname{Tr}\left[\Omega_{\mathbf{m}}(f)^{*} A\right], \quad \forall A \in \mathcal{L}^{2}\left(\mathcal{H}_{\chi}\right), \quad \forall f \in L^{2}(\mathbb{S}) .
$$

Hence, the symbol map is formally given by

$$
\sigma_{\mathbf{m}}[A](x)=\operatorname{Tr}\left[A \Omega_{\underline{s}_{e}^{\star}} \overline{\mathbf{m}}(x)\right], \quad x \in \mathbb{S} .
$$

Here again, the trace on the right hand side is understood in the distributional sense on $\mathcal{D}(\mathbb{S})$. Note however that when $\mathbf{m}$ is essentially bounded, this expression for the symbol map genuinely holds on $\mathcal{L}^{1}\left(\mathcal{H}_{\chi}\right)$, the ideal of trace-class operators on $\mathcal{H}_{\chi}$. When $\mathbf{m}$ is only locally essentially bounded, it also holds rigorously on the dense subspace of $\mathcal{L}^{2}\left(\mathcal{H}_{\chi}\right)$, consisting of finite linear combinations of rank one operators $|\varphi\rangle\langle\psi|$ with $\psi \in \mathcal{D}(Q)$ and $\varphi \in \mathcal{H}_{\chi}$ arbitrary. 
DeFinition 5.46. Let $(M, s, \omega)$ be an elementary symplectic symmetric space. Assuming that $\mathbf{m} / \mathbf{m}_{0} \in L^{\infty}(Q)$, we then set

$$
B_{\mathbf{m}}: L^{2}(\mathbb{S}) \rightarrow L^{2}(\mathbb{S}), \quad f \mapsto \sigma_{\mathbf{m}} \circ \Omega_{\mathbf{m}}(f),
$$

and we call this linear operator the Berezin transform of the quantization map $\Omega_{\mathrm{m}}$.

REMARK 5.47. The Berezin transform measures the obstruction for the symbol map to be inverse of the quantization map. Said differently, the unitarity of the quantization map on $L^{2}(\mathbb{S})$ is equivalent to the triviality of the associated Berezin transform.

Proposition 5.48. Let $(M, s, \omega)$ be an elementary symplectic symmetric space with $\mathbb{Y}$ Abelian and let $\mathbf{m}$ a Borelian function on $Q$ such that $\mathbf{m} / \mathbf{m}_{0} \in L^{\infty}(Q)$ and such that $\mathbb{Y}$ is Abelian. Then

(i) The Berezin transform is a positive and bounded operator on $L^{2}(\mathbb{S})$, with

$$
\left\|B_{\mathbf{m}}\right\| \leq\left\|\mathbf{m} / \mathbf{m}_{0}\right\|_{\infty}^{2} .
$$

(ii) The Berezin transform is a kernel operator with distributional kernel given by

$$
B_{\mathbf{m}}\left[x_{1}, x_{2}\right]=\operatorname{Tr}\left[\Omega_{\underline{s}_{e}^{\star}} \overline{\mathbf{m}}\left(x_{1}\right) \Omega_{\mathbf{m}}\left(x_{2}\right)\right],
$$

and the latter can be identified with

$$
\begin{aligned}
& B_{\mathbf{m}}\left[x_{1}, x_{2}\right]=\delta_{q_{1}}\left(q_{2}\right) \times \int_{\mathfrak{q}} \frac{|\mathbf{m}|^{2}}{\mathbf{m}_{\mathbf{0}}^{2}}\left(\Psi^{-1}(w)\right) e^{i\left\langle\xi,\left[w, \log b_{1}-\log b_{2}\right]\right\rangle} \mathrm{d} w, \\
& \text { where } x_{j}=q_{j} b_{j} \in \mathbb{S}, j=1,2 .
\end{aligned}
$$

Proof. Note that by construction $B_{\mathbf{m}}=\Omega_{\mathbf{m}}^{*} \circ \Omega_{\mathbf{m}}$, yielding positivity and boundedness from boundedness of $\Omega_{\mathbf{m}}$. The operator norm estimate comes from those of Theorem 5.43. The second claim comes from the computation done in the proof of this Theorem.

For $\mathbf{m}=\mathbf{m}_{0}$, the symbol map $\sigma_{\mathbf{m}}$ is the inverse of the quantization map $\Omega_{\mathbf{m}}$. In particular, the associated Berezin transform is trivial. A $\tilde{G}$-equivariant associative product $\star_{\mathbf{m}_{0}}$ on $L^{2}(\mathbb{S})$ is then defined:

$$
f_{1} \star_{\mathbf{m}_{0}} f_{2}:=\sigma_{\mathbf{m}_{0}}\left[\Omega_{\mathbf{m}_{0}}\left(f_{1}\right) \Omega_{\mathbf{m}_{0}}\left(f_{2}\right)\right], \quad \forall f_{1}, f_{2} \in L^{2}(\mathbb{S}) .
$$

We deduce from (5.13) that the product (5.14) is a three-point kernel product, with distributional kernel given by the operator trace of a product of three $\Omega$ 's:

$$
f_{1} \star_{\mathbf{m}_{0}} f_{2}(x)=\int_{\mathbb{S} \times \mathbb{S}} f_{1}(y) f_{2}(z) \operatorname{Tr}\left[\Omega_{\underline{\underline{e}}^{\star} \mathbf{m}_{0}}(x) \Omega_{\mathbf{m}_{0}}(y) \Omega_{\mathbf{m}_{0}}(z)\right] \mathrm{d}_{\mathbb{S}}(y) \mathrm{d}_{\mathbb{S}}(z) .
$$

We will return to the explicit form of the three-point kernel and its geometric interpretation in the next section.

We now come to an important point. Putting together Remark 5.42 and Theorem 5.43, we see that in general the quantization map $\Omega_{\mathbf{m}}$ need not be unitary and involution preserving (the complex conjugation on $L^{2}(\mathbb{S})$ and the adjoint on $\mathcal{L}^{2}\left(\mathcal{H}_{\chi}\right)$ ) at the same time. However, in most cases (e.g. for elementary normal $\mathbf{j}$-groups), the function $\mathbf{m}_{0}$ is $\underline{s}_{e}$-invariant, which implies that the complex conjugation is an involution of the Hilbert algebra $\left(L^{2}(\mathbb{S}), \star_{\mathbf{m}_{0}}\right)$ : 
Proposition 5.49. Let $(M, s, \omega)$ be an elementary symplectic symmetric space with $\mathbb{Y}$ Abelian. Assuming further that $\underline{s}_{e}^{\star} \mathbf{m}_{0}=\mathbf{m}_{0}$, then for all $f_{1}, f_{2} \in L^{2}(\mathbb{S})$, we have:

$$
\overline{f_{1} \star \mathbf{m}_{0} f_{2}}=\overline{f_{2}} \star_{\mathbf{m}_{0}} \overline{f_{1}} .
$$

Next, we pass to a possible approach 2 to define a $\star$-product for the quantization map $\Omega_{\mathbf{m}}$ in the more general context of an arbitrary function $\mathbf{m}$ (and without the assumption that $\mathbb{Y}$ is Abelian).

DEFinition 5.50. Let $(M, s, \omega)$ be a polarized, local and elementary symplectic symmetric space and fix $\mathbf{m}$ a locally essentially bounded Borelian function on $Q$. We then let $L_{\mathbf{m}}^{2}(\mathbb{S})$, be the Hilbert-space of classes of measurable functions on $\mathbb{S}$ for which the norm underlying the following scalar product is finite 3 :

$$
\begin{aligned}
\left\langle f_{1}, f_{2}\right\rangle_{\mathbf{m}}:=\int_{Q \times \mathfrak{q}} \frac{|\mathbf{m}|^{2}}{\mathbf{m}_{\mathbf{0}}^{2}}\left(\Psi^{-1}(w)\right) & \left(\int_{\mathbb{Y}} \bar{f}_{1}\left(q b_{1}\right) e^{-i\left\langle\xi,\left[w, \log b_{1}\right]\right\rangle} \mathrm{d}_{\mathbb{Y}}\left(b_{1}\right)\right) \\
& \times\left(\int_{\mathbb{Y}} f_{2}\left(q b_{2}\right) e^{i\left\langle\xi,\left[w, \log b_{2}\right]\right\rangle} \mathrm{d}_{\mathbb{Y}}\left(b_{2}\right)\right) \mathrm{d}_{Q}(q) \mathrm{d} w .
\end{aligned}
$$

REMARK 5.51. Formally, we have

$$
\left\langle f_{1}, f_{2}\right\rangle_{\mathbf{m}}=\left\langle f_{1}, B_{\mathbf{m}} f_{2}\right\rangle=\operatorname{Tr}\left[\Omega_{\mathbf{m}}\left(f_{1}\right)^{*} \Omega_{\mathbf{m}}\left(f_{2}\right)\right]
$$

where $\langle.,$.$\rangle denotes the inner product of L^{2}(\mathbb{S})$ and $\operatorname{Tr}$ is the operator trace on $\mathcal{H}_{\chi}$.

Repeating the computations done in the proof of Theorem 5.43. we deduce following extension of the latter:

Proposition 5.52. Let $(M, s, \omega)$ be an elementary symplectic symmetric space and let $\mathbf{m}$ be a locally essentially bounded Borelian function on $Q$.

(i) The quantization map $\Omega_{\mathbf{m}}$ is a unitary operator from $L_{\mathbf{m}}^{2}\left(\mathbb{S}, \mathrm{d}_{\mathbb{S}}\right)$ to $\mathcal{L}^{2}\left(\mathcal{H}_{\chi}\right)$,

(ii) Associated to the quantization map $\Omega_{\mathbf{m}}$, there is a deformed product $\star_{\mathbf{m}}$ on $L_{\mathbf{m}}^{2}\left(\mathbb{S}, \mathrm{d}_{\mathbb{S}}\right)$, which is formally given by:

$$
f_{1} \star_{\mathbf{m}} f_{2}=B_{\mathbf{m}}^{-1} \circ \sigma_{\mathbf{m}}\left[\Omega_{\mathbf{m}}\left(f_{1}\right) \Omega_{\mathbf{m}}\left(f_{2}\right)\right],
$$

(iv) $\left(L_{\mathbf{m}}^{2}(\mathbb{S}), \star_{\mathbf{m}}\right)$ is a Hilbert algebra and the complex conjugation is an involution when $\underline{s}_{e}^{\star} \overline{\mathbf{m}}=\mathbf{m}$.

\subsection{The three-point kernel}

The aim of this section is to compute the distributional three-point kernel $\operatorname{Tr}\left[\Omega_{\mathbf{m}_{0}}(x) \Omega_{\mathbf{m}_{0}}(y) \Omega_{\mathbf{m}_{0}}(z)\right]$ of the product $\star_{\mathbf{m}_{0}}$ given in (5.14). We start with two preliminary results extracted from [34]:

THEOREM 5.53. Let $(M, s, \omega)$ be an elementary symplectic symmetric space. Given three points $q_{0}, q_{1}, q_{2}$ in $Q$, the equation

$$
\underline{s}_{q_{2}} \underline{s}_{q_{1}} \underline{s}_{q_{0}}(q)=q
$$

admits a unique solution $q \equiv q\left(q_{0}, q_{1}, q_{2}\right) \in Q$. In particular, this yields a welldefined map

$$
Q^{3} \rightarrow Q^{3}:\left(q_{0}, q_{1}, q_{2}\right) \mapsto\left(q, \underline{s}_{q_{0}}(q), \underline{s}_{q_{1}} \underline{s}_{q_{0}}(q)\right)
$$

${ }^{2}$ This is however not the approach we will follow for the symmetric spaces underlying elementary normal $\mathbf{j}$-groups-see Proposition 6.19

${ }^{3}$ We do not exclude the possibility that $L_{\mathbf{m}}^{2}(\mathbb{S})$ be trivial. 
The latter is a global diffeomorphism called the medial triangle map whose inverse is given by:

$$
\Phi_{Q}: Q^{3} \rightarrow Q^{3}, \quad\left(q_{0}, q_{1}, q_{2}\right) \mapsto\left(\operatorname{mid}\left(q_{0}, q_{1}\right), \operatorname{mid}\left(q_{1}, q_{2}\right), \operatorname{mid}\left(q_{2}, q_{0}\right)\right) .
$$

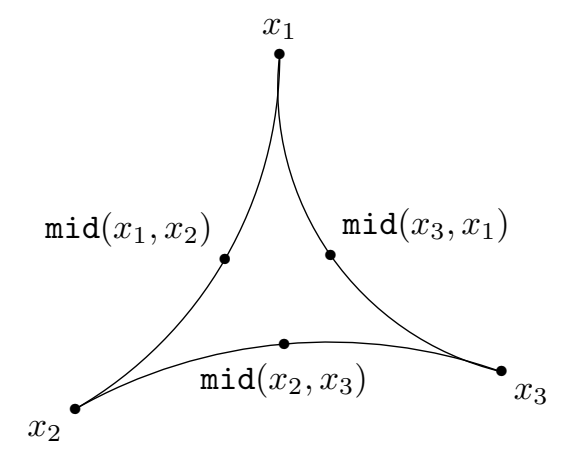

FIGURE 1. The medial triangle of three points

LEMMA 5.54. Let us consider the map

$$
\nu: Q^{3} \rightarrow Q^{3}:\left(q_{0}, q_{1}, q_{2}\right) \mapsto\left(\operatorname{mid}\left(e, q_{0}^{-1} q_{1}\right), \operatorname{mid}\left(e, q_{1}^{-1} q_{2}\right), \operatorname{mid}\left(e, q_{2}^{-1} q_{0}\right)\right) .
$$

Then

$$
\nu \circ \Phi_{Q}^{-1}\left(q_{0}, q_{1}, q_{2}\right)=\left(q^{-1} q_{0},\left(\underline{s}_{q_{0}} q\right)^{-1} q_{1},\left(\underline{s}_{q_{1}} \underline{s}_{q_{0}} q\right)^{-1} q_{2}\right),
$$

where $q$ is the unique fixed point of $\underline{s}_{q_{2}} \underline{s}_{q_{1}} \underline{s}_{q_{0}}$.

Proof. We have $\operatorname{mid}\left(e, q_{0}^{-1} q_{1}\right)=q_{0}^{-1} \operatorname{mid}\left(q_{0}, q_{1}\right)$ and similarly for the other points. Therefore setting

$$
\left(q_{0}^{\prime}, q_{1}^{\prime}, q_{2}^{\prime}\right):=\left(\operatorname{mid}\left(q_{0}, q_{1}\right), \operatorname{mid}\left(q_{1}, q_{2}\right), \operatorname{mid}\left(q_{2}, q_{0}\right)\right)=\Phi_{Q}\left(q_{0}, q_{1}, q_{2}\right),
$$

we get

$$
\nu\left(q_{0}, q_{1}, q_{2}\right)=\left(q_{0}^{-1} q_{0}^{\prime}, q_{1}^{-1} q_{1}^{\prime}, q_{2}^{-1} q_{2}^{\prime}\right) .
$$

A quick look at the above figure leads to $\underline{s}_{q_{2}^{\prime}} \underline{s}_{q_{1}^{\prime}} \underline{\underline{s}}_{q_{0}^{\prime}}\left(q_{0}\right)=q_{0}$ and the assertion immediately follows.

TheOREM 5.55. Let $(M, s, \omega)$ be an elementary symplectic symmetric space with $\mathbb{Y}$ Abelian. Assume that $\mathrm{Jac}_{\underline{s}^{e}}$ is invariant under the inversion map on $Q$. Let $q \equiv q\left(q_{0}, q_{1}, q_{2}\right)$ denote the unique solution of the equation $\underline{s}_{q_{2}} \underline{s}_{q_{1}} \underline{s}_{q_{0}}(q)=q$ in $Q$. Set

$$
\mathcal{J}\left(q_{0}, q_{1}, q_{2}\right):=\left|\operatorname{Jac}_{\left(\underline{s}^{e}\right)^{-1}}\right|\left(q_{0}^{-1} q_{1}\right)\left|\operatorname{Jac}_{\left(\underline{s}^{e}\right)^{-1}}\right|\left(q_{1}^{-1} q_{2}\right)\left|\operatorname{Jac}_{\left(\underline{s}^{e}\right)^{-1}}\right|\left(q_{2}^{-1} q_{0}\right) .
$$

Then, one has:

$$
\underline{s}_{e}^{\star} \overline{\mathbf{m}_{0}}=\mathbf{m}_{0},
$$

and the kernel of the product $\star_{\mathbf{m}_{0}}$ (5.14) is given by:

$$
\begin{aligned}
& K_{\mathbf{m}_{0}}\left(x_{0}, x_{1}, x_{2}\right)=\mathcal{J}^{1 / 2}\left(\Phi_{Q}^{-1}\left(q_{0}, q_{1}, q_{2}\right)\right)\left|\mathrm{Jac}_{\Phi_{Q}^{-1}}\right|\left(q_{0}, q_{1}, q_{2}\right) \\
& \times\left|\mathrm{Jac}_{\Psi}\right|^{1 / 2}\left(q_{0}^{-1} q\right)\left|\mathrm{Jac}_{\Psi}\right|^{1 / 2}\left(q_{1}^{-1} \underline{s}_{q_{0}} q\right)\left|\mathrm{Jac}_{\Psi}\right|^{1 / 2}\left(q_{2}^{-1} \underline{s}_{q_{1}} \underline{s}_{q_{0}} q\right) \\
& \times \mathbf{E}\left(q^{-1} x_{0}\right) \mathbf{E}\left(\left(\underline{s}_{q_{0}} q\right)^{-1} x_{1}\right) \mathbf{E}\left(\left(\underline{s}_{q_{1}} \underline{s}_{q_{0}} q\right)^{-1} x_{2}\right) .
\end{aligned}
$$


PROOF. Under the assumption that $\mathrm{Jac}_{\underline{s}}$ is invariant under the inversion map on $Q, \mathbf{m}_{0}$ is $\underline{s}_{e}$-invariant and reads:

$$
\mathbf{m}_{0}(q)=\left|\operatorname{Jac}_{\underline{s}^{e}}(q) \operatorname{Jac}_{\Psi}(q)\right|^{1 / 2} .
$$

Accordingly, for $f \in L^{2}(\mathbb{S})$, we get from Corollary 5.39 the following expression for the operator kernel of $\Omega_{\mathbf{m}_{0}}(f)$ :

$$
\begin{aligned}
\Omega_{\mathbf{m}_{\mathbf{0}}}(f)\left[q_{0}, q\right]=\left|\mathrm{Jac}_{\Psi}\right|^{1 / 2}\left(\operatorname{mid}\left(e, q_{0}^{-1} q\right)^{-1}\right)\left|\operatorname{Jac}_{\left(\underline{s}^{e}\right)^{-1}}\right|^{1 / 2}\left(q_{0}^{-1} q\right) \\
\times \int_{\mathbb{Y}} f\left(\operatorname{mid}\left(q_{0}, q\right) b\right) \mathbf{E}\left(\operatorname{mid}\left(e, q_{0}^{-1} q\right) b\right) \mathrm{d}_{\mathbb{Y}}(b) .
\end{aligned}
$$

Since for $f \in L^{2}(\mathbb{S}), \Omega_{\mathbf{m}_{0}}(f)$ is Hilbert-Schmidt, the product of three $\Omega_{\mathbf{m}_{0}}(f)$ 's is a fortiori trace-class and thus we can employ the formula:

$$
\begin{aligned}
& \operatorname{Tr}\left[\Omega_{\mathbf{m}_{\mathbf{0}}}\left(f_{0}\right) \Omega_{\mathbf{m}_{\mathbf{0}}}\left(f_{1}\right) \Omega_{\mathbf{m}_{\mathbf{0}}}\left(f_{2}\right)\right]= \\
& \int_{Q^{3}} \Omega_{\mathbf{m}_{\mathbf{0}}}\left(f_{0}\right)\left[q_{0}, q_{1}\right] \Omega_{\mathbf{m}_{\mathbf{0}}}\left(f_{1}\right)\left[q_{1}, q_{2}\right] \Omega_{\mathbf{m}_{\mathbf{0}}}\left(f_{2}\right)\left[q_{2}, q_{0}\right] \mathrm{d}_{Q}\left(q_{0}\right) \mathrm{d}_{Q}\left(q_{1}\right) \mathrm{d}_{Q}\left(q_{2}\right) .
\end{aligned}
$$

Using Theorem 5.53 and the formula above for the kernel of $\Omega_{\mathbf{m}_{0}}\left(f_{j}\right)$, we see that the above trace equals:

$$
\begin{aligned}
& \int_{Q^{3} \times \mathbb{Y}^{3}}\left|\operatorname{Jac}_{\Psi}\right|^{1 / 2}\left(\operatorname{mid}\left(e, q_{0}^{-1} q_{1}\right)^{-1}\right)\left|\operatorname{Jac}_{\Psi}\right|^{1 / 2}\left(\operatorname{mid}\left(e, q_{1}^{-1} q_{2}\right)^{-1}\right) \\
& \times\left|\operatorname{Jac}_{\Psi}\right|^{1 / 2}\left(\operatorname{mid}\left(e, q_{2}^{-1} q_{0}\right)^{-1}\right) \mathcal{J}^{1 / 2}\left(q_{0}, q_{1}, q_{2}\right) f_{0}\left(\operatorname{mid}\left(q_{0}, q_{1}\right) b_{0}\right) f_{1}\left(\operatorname{mid}\left(q_{1}, q_{2}\right) b_{1}\right) \\
& \times f_{2}\left(\operatorname{mid}\left(q_{2}, q_{0}\right) b_{2}\right) \mathbf{E}\left(\operatorname{mid}\left(e, q_{0}^{-1} q_{1}\right) b_{0}\right) \mathbf{E}\left(\operatorname{mid}\left(e, q_{1}^{-1} q_{2}\right) b_{1}\right) \mathbf{E}\left(\operatorname{mid}\left(e, q_{2}^{-1} q_{0}\right) b_{2}\right) \\
& \times \mathrm{d}_{Q}\left(q_{0}\right) \mathrm{d}_{Q}\left(q_{1}\right) \mathrm{d}_{Q}\left(q_{2}\right) \mathrm{d}_{\mathbb{Y}}\left(b_{0}\right) \mathrm{d}_{\mathbb{Y}}\left(b_{1}\right) \mathrm{d}_{\mathbb{Y}}\left(b_{2}\right) .
\end{aligned}
$$

Performing the change of variable $\left(q_{0}, q_{1}, q_{2}\right) \mapsto \Phi_{Q}^{-1}\left(q_{0}, q_{1}, q_{2}\right)$ and setting $x_{j}:=$ $q_{j} b_{j} \in \mathbb{S}, j=0,1,2$, we get

$$
\begin{aligned}
& \int_{\mathbb{S}^{3}}\left|\operatorname{Jac}_{\Psi}\right|^{1 / 2}\left(q_{0}^{-1} q\right)\left|\operatorname{Jac}_{\Psi}\right|^{1 / 2}\left(q_{1}^{-1} \underline{s}_{q_{0}} q\right)\left|\mathrm{Jac}_{\Psi}\right|^{1 / 2}\left(q_{2}^{-1} \underline{s}_{q_{1}} \underline{s}_{q_{0}} q\right) \mathcal{J}^{1 / 2}\left(\Phi_{Q}^{-1}\left(q_{0}, q_{1}, q_{2}\right)\right) \\
& \times\left|\mathrm{Jac}_{\Phi_{Q}^{-1}}\right|\left(q_{0}, q_{1}, q_{2}\right) \mathbf{E}\left(q^{-1} x_{0}\right) \mathbf{E}\left(\left(\underline{s}_{q_{0}} q\right)^{-1} x_{1}\right) \mathbf{E}\left(\left(\underline{s}_{q_{1}} \underline{s}_{q_{0}} q\right)^{-1} x_{2}\right) f_{0}\left(x_{0}\right) f_{1}\left(x_{1}\right) \\
& \times f_{2}\left(x_{2}\right) \mathrm{d}_{\mathbb{S}}\left(x_{0}\right) \mathrm{d}_{\mathbb{S}}\left(x_{1}\right) \mathrm{d}_{\mathbb{S}}\left(x_{2}\right)
\end{aligned}
$$

where $q \equiv q\left(q_{0}, q_{1}, q_{2}\right)$ is the unique solution of the equation $\underline{s}_{q_{2}} \underline{s}_{q_{1}} \underline{s}_{q_{0}}(q)=q$ (see Lemma 5.54). The result then follows by identification.

Last, using Lemma 5.37, we deduce the following expression for the phase in the kernel of the product $\star_{\mathbf{m}_{0}}$ :

COROLlary 5.56. Write $K_{\mathbf{m}_{0}}=A_{\mathbf{m}_{0}} e^{-i S}$ for the three-point kernel given in Proposition 5.55. Then we have for $x_{j}=q_{j} b_{j} \in \mathbb{S}, q_{j} \in Q, b_{j} \in \mathbb{Y}, j=0,1,2$ :

$$
\begin{aligned}
& S\left(x_{0}, x_{1}, x_{2}\right)= \\
& \left\langle\xi,\left[\Psi\left(q_{0}^{-1} q\right), \log b_{0}\right]\right\rangle+\left\langle\xi,\left[\Psi\left(q_{1}^{-1} \underline{s}_{q_{0}} q\right), \log b_{1}\right]\right\rangle+\left\langle\xi,\left[\Psi\left(q_{2}^{-1} \underline{s}_{q_{1}} \underline{s}_{q_{0}} q\right), \log b_{2}\right]\right\rangle,
\end{aligned}
$$

where $q \equiv q\left(q_{0}, q_{1}, q_{2}\right)$ is the unique solution of the equation $\underline{s}_{q_{2}} \underline{s}_{q_{1}} \underline{s}_{q_{0}}(q)=q$ (see Theorem 5.53). 


\subsection{Extensions of polarization quadruples}

We first observe that, given two polarization quadruples $\left(G_{j}, \sigma_{j}, \xi_{j}, B_{j}\right), j=1,2$ (in the general sense of Definition [5.15), a morphism $\phi$ between them yields a $G_{1^{-}}$ equivariant intertwiner:

$$
\phi^{\star}: C^{\infty}\left(G_{2}\right)^{B_{2}} \rightarrow C^{\infty}\left(G_{1}\right)^{B_{1}}
$$

such that

$$
U_{\chi_{1}}\left(g_{1}\right) \phi^{\star} \hat{\varphi}_{2}=\phi^{\star}\left(U_{\chi_{2}}\left(\phi\left(g_{1}\right)\right) \hat{\varphi_{2}}\right) \text {. }
$$

Note that the condition $\phi^{\star} \xi_{2}=\xi_{1}$ (cf. Definition 5.15) implies $\chi_{1}\left(b_{1}\right)=\chi_{2}\left(\phi\left(b_{1}\right)\right.$ ) in view of (5.7).

In the context of the transvection and full polarization quadruples, we observe:

LEMMA 5.57. Let $(M, s, \omega)$ be an elementary symplectic symmetric space, associated to a non-exact polarized transvection triple $(\mathfrak{g}, \sigma, \varpi)$. Consider $\left(\mathbb{L}, \sigma_{\mathbb{L}}, \xi, \mathbf{B}\right)$ and $\left(\tilde{G}, \tilde{\sigma},\left.\xi\right|_{\tilde{G}}, B\right)$ and the full and transvection polarization quadruples as in Definition 5.19. Then, the intertwiner (5.15) corresponding to the injection $\tilde{G} \rightarrow \mathbb{L}$ is a linear isomorphism.

Proof. The injection $j: \tilde{G} \rightarrow \mathbb{L}$ induces a global diffeomorphism $\tilde{G} / B \rightarrow$ $\mathbb{L} / \mathbf{B}, g B \mapsto g \mathbf{B}$. Indeed, the $\operatorname{map} \tilde{G} / \tilde{K} \rightarrow \mathbb{L} / \tilde{\mathbf{D}}: g \tilde{K} \mapsto g \tilde{\mathbf{D}}$ is an identification. Considering the natural projections $\tilde{G} / \tilde{K} \rightarrow \tilde{G} / B$ and $\mathbb{L} / \tilde{\mathbf{D}} \rightarrow \mathbb{L} / \mathbf{B}$, one observes that the diagram

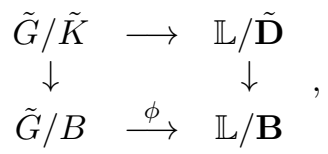

where $\phi(g B):=g \mathbf{B}$, is commutative. In particular, $\phi$ is surjective. Examining its differential proves that is also a submersion. The space $Q=\tilde{G} / B$, being exponential, has trivial fundamental group. The map $\phi$ is therefore a diffeomorphism. Also the restrictions $C^{\infty}(\tilde{G})^{B} \rightarrow C^{\infty}(Q)$ and $C^{\infty}(\mathbb{L})^{\mathbf{B}} \rightarrow C^{\infty}(Q)$ are linear isomorphisms and one observes that $\left.j^{\star} \hat{\varphi}\right|_{Q}=\left.\hat{\varphi}\right|_{Q}$.

Note that when the modular function of $\mathbf{B}$ coincides with the restriction to $\mathbf{B}$ of the modular function of $\mathbb{L}$, then there exists a $\mathbb{L}$-invariant measure on $\mathbb{L} / \mathbf{B}$. From the isomorphism $\mathbb{L} / \mathbf{B} \simeq \tilde{G} / B \simeq Q$, we see that the later is a left-Haar measure on $Q$. Hence, under the assumption above, we deduce that the left-Haar measure $\mathrm{d}_{Q}$ is also $\mathbb{L}$-invariant. This, together with the above Lemma, yields:

LEMMA 5.58. In the setting of Lemma 5.57 and when the modular function of $\mathbf{B}$ coincides with the restriction to $\mathbf{B}$ of the modular function of $\mathbb{L}$, the injection $\tilde{\mathfrak{D}} \rightarrow \mathfrak{L}$ induces a unitary representation $\mathcal{R}: \tilde{\mathbf{D}} \rightarrow \mathcal{U}\left(\mathcal{H}_{\chi}\right)$ of the corresponding analytic subgroup $\tilde{\mathbf{D}} \subset \mathbb{L}$ on the representation space $\mathcal{H}_{\chi}$ associated to the transvection quadruple $(\tilde{G}, \tilde{\sigma}, \xi, B)$.

Consider now two Lie groups $G_{j}, j=1,2$, with unitary representations $\left(U_{j}, \mathcal{H}_{j}\right)$, together with a Lie group homomorphism

$$
\rho: G_{1} \rightarrow G_{2},
$$

and form the associated semi-direct product $G_{1} \ltimes_{\mathbf{R}} G_{2}$, where

$$
\mathbf{R}_{g_{1}}\left(g_{2}\right):=\mathbf{C}_{\rho\left(g_{1}\right)}\left(g_{2}\right)=\rho\left(g_{1}\right) g_{2} \rho\left(g_{1}\right)^{-1}, \quad \forall g_{j} \in G_{j} .
$$


We deduce the representation homomorphism:

$$
\mathcal{R}: G_{1} \rightarrow \mathcal{U}\left(\mathcal{H}_{2}\right), \quad g_{1} \mapsto U_{2}\left(\rho\left(g_{1}\right)\right) .
$$

Within this setting, we first observe:

Lemma 5.59. Parametrizing an element $g \in G_{1} \ltimes_{\mathbf{R}} G_{2}$ as $g=g_{1} . g_{2}$, the map

$$
U: G_{1} \ltimes_{\mathbf{R}} G_{2} \rightarrow \mathcal{U}\left(\mathcal{H}_{1} \otimes \mathcal{H}_{2}\right), \quad g \mapsto U_{1}\left(g_{1}\right) \otimes \mathcal{R}\left(g_{1}\right) U_{2}\left(g_{2}\right),
$$

defines a unitary representation of $G_{1} \ltimes_{\mathbf{R}} G_{2}$ on the tensor product Hilbert space $\mathcal{H}:=\mathcal{H}_{1} \otimes \mathcal{H}_{2}$.

Proof. Let $g_{j}, g_{j}^{\prime} \in G_{j}, j=1,2$. Then, on the first hand:

$$
\begin{aligned}
U\left(g_{1} g_{2} \cdot g_{1}^{\prime} g_{2}^{\prime}\right)=U\left(g_{1} g_{1}^{\prime} \mathbf{R}_{g_{1}^{\prime-1}}\left(g_{2}\right) g_{2}^{\prime}\right) & =U_{1}\left(g_{1} g_{1}^{\prime}\right) \otimes \mathcal{R}\left(g_{1} g_{1}^{\prime}\right) U_{2}\left(\mathbf{R}_{g_{1}^{\prime}-1}\left(g_{2}\right) g_{2}^{\prime}\right) \\
& =U_{1}\left(g_{1} g_{1}^{\prime}\right) \otimes \mathcal{R}\left(g_{1}\right) U_{2}\left(g_{2}\right) \mathcal{R}\left(g_{1}^{\prime}\right) U_{2}\left(g_{2}^{\prime}\right),
\end{aligned}
$$

while on the second hand:

$$
\begin{aligned}
U\left(g_{1} g_{2}\right) U\left(g_{1}^{\prime} g_{2}^{\prime}\right) & =\left(U_{1}\left(g_{1}\right) \otimes \mathcal{R}\left(g_{1}\right) U_{2}\left(g_{2}\right)\right)\left(U_{1}\left(g_{1}^{\prime}\right) \otimes \mathcal{R}\left(g_{1}^{\prime}\right) U_{2}\left(g_{2}^{\prime}\right)\right) \\
& =U_{1}\left(g_{1} g_{1}^{\prime}\right) \otimes \mathcal{R}\left(g_{1}\right) U_{2}\left(g_{2}\right) \mathcal{R}\left(g_{1}^{\prime}\right) U_{2}\left(g_{2}^{\prime}\right)
\end{aligned}
$$

and the proof is complete.

Consider Lastly two full polarization quadruples $\left(\mathbb{L}_{j}, \sigma_{j}, \xi_{j}, \mathbf{B}_{j}\right), j=1,2$, associated to two local symplectic symmetric spaces $\left(M_{j}, s_{j}, \omega_{j}\right)$. Let also $\left(U_{\chi_{j}}, \mathcal{H}_{\chi_{j}}, \Omega_{j}\right)$ be the unitary representation of $\mathbb{L}_{j}$ and of the representation of the symplectic symmetric space $M_{j}=\tilde{G}_{j} / \tilde{K}_{j}=\mathbb{L}_{j} / \tilde{\mathbf{D}}_{j}$ (see Remark 5.29). Finally, let $\mathbf{K}$ be a Lie subgroup of $\mathbb{L}_{1}$ that acts transitively on $M_{1}$, and consider the associated K-equivariant diffeomorphism:

$$
\varphi: \mathbf{K} /\left(\mathbf{K} \cap \tilde{\mathbf{D}}_{1}\right) \rightarrow M_{1}
$$

Now, given a Lie group homomorphism $\rho: \mathbf{K} \rightarrow \tilde{\mathbf{D}}_{2} \subset \mathbb{L}_{2}$, we can form the semidirect product $\mathbf{K} \ltimes_{\mathbf{R}} \mathbb{L}_{2}$, according to (5.16). Under these conditions, we have the global identification:

$$
\begin{aligned}
\left(\mathbf{K} \ltimes_{\mathbf{R}} \mathbb{L}_{2}\right) /\left(\left(\mathbf{K} \cap \tilde{\mathbf{D}}_{1}\right) \ltimes_{\mathbf{R}} \tilde{\mathbf{D}}_{2}\right) & \rightarrow M_{1} \times M_{2}, \\
\left(g_{1} . g_{2}\right)\left(\mathbf{K} \cap \tilde{\mathbf{D}}_{1}\right) \ltimes_{\mathbf{R}} \tilde{\mathbf{D}}_{2} & \mapsto\left(\varphi\left(g_{1} \mathbf{K} \cap \tilde{\mathbf{D}}_{1}\right), g_{2} \tilde{\mathbf{D}}_{2}\right) .
\end{aligned}
$$

Proposition 5.60. Let $U$ be the unitary representation of $\mathbf{K} \ltimes_{\mathbf{R}} \mathbb{L}_{2}$ on $\mathcal{H}:=$ $\mathcal{H}_{\chi_{1}} \otimes \mathcal{H}_{\chi_{2}}$ constructed in Lemma 5.59. Then under the conditions displayed above,

(i) the map

$$
\boldsymbol{\Omega}: \mathbf{K} \ltimes_{\mathbf{R}} \mathbb{L}_{2} \rightarrow \mathcal{U}_{s a}(\mathcal{H}), \quad g \mapsto U(g) \circ\left(\Sigma_{1} \otimes \Sigma_{2}\right) \circ U(g)^{*},
$$

is constant on the left cosets of $\left(\mathbf{K} \cap \tilde{\mathbf{D}}_{1}\right) \ltimes_{\mathbf{R}} \tilde{\mathbf{D}}_{2}$ in $\mathbf{K} \ltimes_{\mathbf{R}} \mathbb{L}_{2}$.

(ii) For every $g_{1} \in \mathbf{K}$ and $g_{2} \in \mathbb{L}_{2}$, one ha屯

$$
\boldsymbol{\Omega}\left(g_{2} . g_{1}\right)=\Omega_{1}\left(g_{1}\right) \otimes \Omega_{2}\left(g_{2}\right) .
$$

(iii) Under the identification (5.18), the quotient map

$$
\Omega: M_{1} \times M_{2} \rightarrow \mathcal{U}_{s a}(\mathcal{H}),
$$

is $\mathbf{K} \rtimes_{\mathbf{R}} \mathbb{L}_{2}$-equivariant.

\footnotetext{
${ }^{4}$ Warning: the reverse order in the group elements.
} 
Proof. We start by checking item (ii). Observe first that

$$
\begin{aligned}
U\left(g_{2} g_{1}\right)=U\left(g_{1} \mathbf{R}_{g_{1}^{-1}} g_{2}\right) & =U_{\chi_{1}}\left(g_{1}\right) \otimes \mathcal{R}\left(g_{1}\right) U_{\chi_{2}}\left(\mathbf{R}_{g_{1}^{-1}} g_{2}\right) \\
& =U_{\chi_{1}}\left(g_{1}\right) \otimes U_{\chi_{2}}\left(g_{2}\right) \mathcal{R}\left(g_{1}\right) .
\end{aligned}
$$

Now, since $\rho$ is $\tilde{\mathbf{D}}_{2}$-valued, we have for all $g_{1} \in \mathbf{K}$ :

$$
\mathcal{R}\left(g_{1}\right) \Sigma_{2} \mathcal{R}\left(g_{1}\right)^{*}=U_{\chi_{2}}\left(\rho\left(g_{1}\right)\right) \Sigma_{2} U_{\chi_{2}}\left(\rho\left(g_{1}\right)\right)^{*}=\Sigma_{2},
$$

(cf. Lemma 5.11). Moreover, one has

$$
\begin{aligned}
\boldsymbol{\Omega}\left(g_{2} g_{1}\right) & =\left(U_{\chi_{1}}\left(g_{1}\right) \otimes U_{\chi_{2}}\left(g_{2}\right) \mathcal{R}\left(g_{1}\right)\right) \circ\left(\Sigma_{1} \otimes \Sigma_{2}\right) \circ\left(U_{\chi_{1}}\left(g_{1}\right)^{*} \otimes \mathcal{R}\left(g_{1}\right)^{*} U_{\chi_{2}}\left(g_{2}\right)^{*}\right) \\
& =U_{\chi_{1}}\left(g_{1}\right) \Sigma_{1} U_{\chi_{1}}\left(g_{1}\right)^{*} \otimes U_{\chi_{2}}\left(g_{2}\right) \mathcal{R}\left(g_{1}\right) \Sigma_{2} \mathcal{R}\left(g_{1}\right)^{*} U_{\chi_{2}}\left(g_{2}\right)^{*} \\
& =\Omega_{1}\left(g_{1}\right) \otimes \Omega_{2}\left(g_{2}\right) .
\end{aligned}
$$

This implies (ii) and (i) consequently. Regarding item (iii), one observes at the level of $\mathbf{K} \ltimes_{\mathbf{R}} \mathbb{L}_{2}$ that

$$
\boldsymbol{\Omega}\left(g g^{\prime}\right)=U(g) \boldsymbol{\Omega}\left(g^{\prime}\right) U(g)^{*},
$$

which is enough to conclude.

REMARK 5.61. In the same manner as in Definition 5.27, given a Borelian function $\mathbf{m}$ on the product manifold $\left(\mathbb{L}_{1} / \tilde{\mathbf{D}}_{1}\right) \times\left(\mathbb{L}_{2} / \tilde{\mathbf{D}}_{2}\right)$, we may define for $g \in$ $\mathbf{K} \ltimes_{\mathbf{R}} \mathbb{L}_{2}:$

$$
\boldsymbol{\Omega}_{\mathbf{m}}(g):=U(g) \circ \mathbf{m} \circ\left(\Sigma_{1} \otimes \Sigma_{2}\right) \circ U(g)^{*} .
$$

Of course, the above procedure can be iterated, namely one observes:

Proposition 5.62. Let $\left(\mathbb{L}_{j}, \sigma_{j}, \xi_{j}, \mathbf{B}_{j}\right), j=1, \ldots, N$, be $N$ full polarization quadruples, associated to $N$ elementary symplectic symmetric spaces $\left(M_{j}, s_{j}, \omega_{j}\right)$ satisfying the extra conditions of coincidence of the modular function on $\mathbf{B}_{j}$ with the restriction to $\mathbf{B}_{j}$ of the modular function of $\mathbb{L}_{j}$, according to Lemmas 5.57 and 5.58. For every $j=1, \ldots, N-1$, consider a subgroup $\mathbf{K}_{j}$ that acts transitively on $M_{j}$ together with a Lie group homomorphism $\rho_{j}: \mathbf{K}_{j} \rightarrow \tilde{\mathbf{D}}_{j+1}$. Set $\mathbf{K}_{N}:=\mathbb{L}_{N}$, assume that for every such $j$, the subgroup $\rho_{j}\left(\mathbf{K}_{j}\right)$ normalizes $\mathbf{K}_{j+1}$ in $\mathbb{L}_{j+1}$ and denote by $\mathbf{R}_{j}$ the corresponding homomorphism from $\mathbf{K}_{j}$ to $\operatorname{Aut}\left(\mathbf{K}_{j+1}\right)$. Then, iterating the procedure described in Proposition 5.60 yields a map

$$
\boldsymbol{\Omega}: M_{1} \times M_{2} \times \cdots \times M_{N} \rightarrow \mathcal{U}_{s a}(\mathcal{H}),
$$

into the self-adjoint unitaries on the product Hilbert space $\mathcal{H}:=\mathcal{H}_{\chi_{1}} \otimes \cdots \otimes \mathcal{H}_{\chi_{N}}$ that is equivariant under the natural action of the Lie group

$$
\left(\ldots\left(\left(\mathbf{K}_{1} \ltimes_{\mathbf{R}_{1}} \mathbf{K}_{2}\right) \ltimes_{\mathbf{R}_{2}} \mathbf{K}_{3}\right) \ltimes_{\mathbf{R}_{3}} \ldots\right) \ltimes_{\mathbf{R}_{N-1}} \mathbb{L}_{N} .
$$

This 'elementary' tensor product construction for the quantization map on direct products of polarized symplectic symmetric spaces (but with covariance under semi-direct products of subgroups of the covariance group of each piece) allows to transfer most of the results of the previous sections. For notational convenience, we formulate all that follows in the context of two elementary pieces, i.e. in the context of Proposition 5.60 rather than in the context of Proposition 5.62

So in all that follows, we assume we are given two elementary symplectic symmetric spaces $\left(M_{j}, s_{j}, \omega_{j}\right), j=1,2$ (see Definition 5.35). We also let $\mathbb{S}_{j}=Q_{j} \ltimes \mathbb{Y}_{j}$, $j=1,2$, be the subgroups of $\tilde{G}_{j}$ (and thus of $\mathbb{L}_{j}$ ) that acts simply transitively on $M_{j}$. We also assume that we are given a homomorphism $\rho: \mathbb{S}_{1} \rightarrow \tilde{\mathbf{D}}_{2}$ (i.e. 
the role of $\mathbf{K}$ in Proposition 5.60 is played by $\mathbb{S}_{1}$ ). In this particular context, the identification (5.18) becomes:

$$
\mathbb{S}_{1} \ltimes_{\mathbf{R}} \mathbb{S}_{2} \rightarrow M_{1} \times M_{2}, \quad g_{1} . g_{2} \mapsto\left(g_{1} \tilde{\mathbf{D}}_{1}, g_{2} \tilde{\mathbf{D}}_{2}\right) .
$$

We also let $\mathbf{m}_{0}:=\mathbf{m}_{0}^{1} \otimes \mathbf{m}_{0}^{2}$ be the smooth function on $Q_{1} \times Q_{2}$, where $\mathbf{m}_{0}^{j}$ is the function on $Q_{j}$ given in (5.12). Combining Proposition 5.60 with the results of sections 5.3, 5.4, 5.5] and 5.6, we eventually obtain:

Theorem 5.63. Let $\left(M_{j}, s_{j}, \omega_{j}\right), j=1,2$, be two elementary symplectic symmetric spaces and consider an homomorphism $\rho: \mathbb{S}_{1} \rightarrow \tilde{\mathbf{D}}_{2}$. Within the notations given above, we have:

(i) Identifying $\mathcal{H}_{\chi_{1}} \otimes \mathcal{H}_{\chi_{2}}$ with $L^{2}\left(Q_{1} \times Q_{2}\right)$ and parametrizing an element $g \in$ $\mathbb{S}_{1} \ltimes_{\mathbf{R}} \mathbb{S}_{2}$ as $g=q_{2} b_{2} q_{1} b_{1}, q_{j} \in Q_{j}, b_{j} \in \mathbb{Y}_{j}$, we have for $\varphi \in \mathcal{D}\left(Q_{1} \times Q_{2}\right)$ :

$\boldsymbol{\Omega}_{\mathbf{m}_{0}}(g) \varphi\left(\bar{q}_{1}, \bar{q}_{2}\right)=$

$\mathbf{m}_{0}^{1}\left(q_{1}^{-1} \bar{q}_{1}\right) \mathbf{m}_{0}^{2}\left(q_{2}^{-1} \bar{q}_{2}\right) \mathbf{E}^{\mathbb{S}_{1} \ltimes \mathbb{S}_{2}}\left(\bar{q}_{1}^{-1} q_{1} b_{1}, \bar{q}_{2}^{-1} q_{2} b_{2}\right) \varphi\left(\underline{s}_{q_{1}} \bar{q}_{1}, \underline{s}_{2} q_{2} \bar{q}_{2}\right)$, where

$\mathbf{E}^{\mathbb{S}_{1} \ltimes \mathbb{S}_{2}}\left(q_{1} b_{1}, q_{2} b_{2}\right):=\mathbf{E}^{\mathbb{S}_{1}}\left(q_{1} b_{1}\right) \mathbf{E}^{\mathbb{S}_{2}}\left(q_{2} b_{2}\right), \quad \forall q_{j} \in Q_{j}, \forall b_{j} \in \mathbb{Y}_{j}$, and $\mathbf{E}^{\mathbb{S}_{j}}, j=1,2$, is the one-point phase attached to each elementary symplectic symmetric space $M_{j}$ as given in Lemma 5.32.

(ii) Moreover, when $\mathbb{Y}_{1}$ and $\mathbb{Y}_{2}$ are Abelian, the map

$$
\begin{aligned}
\boldsymbol{\Omega}_{\mathbf{m}_{0}}: L^{2}\left(\mathbb{S}_{1} \ltimes_{\mathbf{R}} \mathbb{S}_{2}\right) & \rightarrow \mathcal{L}^{2}\left(\mathcal{H}_{\chi_{1}} \otimes \mathcal{H}_{\chi_{2}}\right), \\
f & \mapsto \int_{\mathbb{S}_{1} \ltimes_{\mathbf{R}} \mathbb{S}_{2}} f(g) \boldsymbol{\Omega}_{\mathbf{m}_{0}}(g) \mathrm{d}_{\mathbb{S}_{1} \ltimes_{\mathbf{R}} \mathbb{S}_{2}}(g),
\end{aligned}
$$

is unitary and $\mathbb{S}_{1} \ltimes_{\mathbf{R}} \mathbb{S}_{2}$ equivariant.

(iii) Denoting by $\sigma_{\mathbf{m}_{0}}$ the adjoint of $\boldsymbol{\Omega}_{\mathbf{m}_{0}}$, the associated deformed product:

$$
f_{1} \star_{\mathbf{m}_{0}} f_{2}:=\sigma_{\mathbf{m}_{0}}\left[\boldsymbol{\Omega}_{\mathbf{m}_{0}}\left(f_{1}\right) \boldsymbol{\Omega}_{\mathbf{m}_{0}}\left(f_{2}\right)\right],
$$

takes on $\mathcal{D}\left(\mathbb{S}_{1} \ltimes_{\mathbf{R}} \mathbb{S}_{2}\right)$ the expression

$\int_{\left(\mathbb{S}_{1} \ltimes_{\mathbf{R}} \mathbb{S}_{2}\right)^{2}} K_{\mathbf{m}_{0}}^{\mathbb{S}_{1} \ltimes_{\mathbf{R}} \mathbb{S}_{2}}\left(g, g^{\prime}, g^{\prime \prime}\right) f_{1}\left(g^{\prime}\right) f_{2}\left(g^{\prime \prime}\right) \mathrm{d}_{\mathbb{S}_{1} \ltimes_{\mathbf{R}} \mathbb{S}_{2}}\left(g^{\prime}\right) \mathrm{d}_{\mathbb{S}_{1} \ltimes_{\mathbf{R}} \mathbb{S}_{2}}\left(g^{\prime \prime}\right)$,

where the three-points kernel $K_{\mathbf{m}_{0}}^{\mathbb{S}_{1} \times_{\mathbf{R}} \mathbb{S}_{2}}$ is given, with $g=g_{2} g_{1}, g^{\prime}=g_{2}^{\prime} g_{1}^{\prime}$ and $g^{\prime \prime}=g_{2}^{\prime \prime} g_{1}^{\prime \prime}$ by

$$
K_{\mathbf{m}_{0}}^{\mathbb{S}_{1} \ltimes_{\mathbf{R}} \mathbb{S}_{2}}\left(g, g^{\prime}, g^{\prime \prime}\right):=K_{\mathbf{m}_{0}^{1}}^{\mathbb{S}_{1}}\left(g_{1}, g_{1}^{\prime}, g_{1}^{\prime \prime}\right) K_{\mathbf{m}_{0}^{2}}^{\mathbb{S}_{2}}\left(g_{2}, g_{2}^{\prime}, g_{2}^{\prime \prime}\right),
$$

with $K_{\mathbf{m}_{0}}^{\mathbb{S}_{j}}, j=1,2$, as given in Proposition 5.55 . 


\section{CHAPTER 6}

\section{Quantization of Kählerian Lie groups}

The aim of this chapter is two-fold. First, we establish that the symplectic symmetric space $\left(\mathbb{S}, s, \omega^{\mathbb{S}}\right)$ associated with an elementary normal $\mathbf{j}$-group $\mathbb{S}$ (see section 2.2) underlies an elementary local and polarized symplectic symmetric space, in the sense of Definitions [5.19, 5.30 and 5.35] Using the whole construction of chapter 5. we will then be able to construct a $\mathbb{B}$-equivariant quantization map for any normal $\mathbf{j}$-group $\mathbb{B}$. Second, we will show that the composition law of symbols associated to this quantization map, coincides exactly with the left- $\mathbb{B}$-equivariant star-products on $\mathbb{B}$ that we have considered in chapter 3 ,

\subsection{The transvection quadruple of an elementary normal j-group}

We start by describing the non-exact transvection siLa $(\mathfrak{g}, \sigma, \varpi)$ underlying the symplectic symmetric space structure $\left(\mathbb{S}, s, \omega^{\mathbb{S}}\right)$ of an elementary normal $\mathbf{j}$-group, as described in section 2.2

The transvection group $G$ of $\mathbb{S}$ is the connected and simply connected Lie group whose Lie algebra $\mathfrak{g}$ is a one-dimensional split extension of two copies of the Heisenberg algebra:

$$
\mathfrak{g}:=\mathfrak{a} \ltimes_{\rho}(\mathfrak{h} \oplus \mathfrak{h}),
$$

where, again, $\mathfrak{a}=\mathbb{R} H$ and the extension homomorphism is given by $\rho:=\rho_{\mathfrak{h}} \oplus$ $\left(-\rho_{\mathfrak{h}}\right) \in \operatorname{Der}(\mathfrak{h} \oplus \mathfrak{h})$, with $\rho_{\mathfrak{h}}$ defined in (2.1). The involution $\sigma$ of $\mathfrak{g}$ is given by

$$
\sigma(a H+(X \oplus Y)):=(-a H)+(Y \oplus X), \quad \forall a \in \mathbb{R}, \quad \forall X, Y \in \mathfrak{h} .
$$

One has the associated $( \pm 1)$-eigenspaces decomposition:

$$
\mathfrak{g}=\mathfrak{k} \oplus \mathfrak{p}, \quad \mathfrak{k}:=\mathfrak{h}_{+} \quad \text { and } \quad \mathfrak{p}:=\mathfrak{a} \oplus \mathfrak{h}_{-},
$$

where for every subspace $F \subset \mathfrak{h}$, we set

$$
F_{ \pm}:=\{X \oplus( \pm X), X \in F\} \subset \mathfrak{h} \oplus \mathfrak{h},
$$

and for every element $X \in \mathfrak{h}$ we let $X_{ \pm}:=\frac{1}{2}(X \oplus( \pm X)) \in \mathfrak{h} \oplus \mathfrak{h}$. Last, we define $\varpi \in \Lambda^{2} \mathfrak{g}^{\star}$ by

$$
\varpi\left(H, E_{-}\right)=2 \quad \text { and } \quad \varpi\left(v_{-}, v_{-}^{\prime}\right)=\omega^{0}\left(v, v^{\prime}\right), \quad \forall v, v^{\prime} \in V,
$$

and by zero everywhere else on $\mathfrak{g} \times \mathfrak{g}$. Note that $\varpi$ is $\mathfrak{k}$-invariant and its restriction to $\mathfrak{p}$ is non-degenerate. This implies that $\varpi$ is a Chevalley two-cocycle (see [1]). Also, from $\left[H, \mathfrak{h}_{-}\right]=\mathfrak{h}_{+}=\mathfrak{k}$, we deduce that $[\mathfrak{p}, \mathfrak{p}]=\mathfrak{k}$ and clearly the action of $\mathfrak{k}$ on $\mathfrak{p}$ is faithful. Thus, in terms of Definition [5.4 we have proved the following:

Proposition 6.1. The siLa $(\mathfrak{g}, \sigma, \varpi)$ defined by (6.1), (6.2) and (6.4), is a transvection symplectic triple. 
Consider now $(\tilde{\mathfrak{g}}, \tilde{\sigma}, \delta \xi)$ the exact siLa constructed out of the non-exact siLa $(\mathfrak{g}, \sigma, \varpi)$ as in Lemma 5.11 , Recall that $\tilde{\mathfrak{g}}$ is the one-dimensional central extension of $\mathfrak{g}$ with generator $Z$ and table

$$
[X, Y]_{\mathfrak{g}}=[X, Y]_{\mathfrak{g}}+\varpi(X, Y) Z, \quad \forall X, Y \in \mathfrak{g} .
$$

The involution $\tilde{\sigma}$ equals $\operatorname{id}_{\tilde{\mathfrak{k}}} \oplus\left(-\mathrm{id}_{\mathfrak{p}}\right)$, where $\tilde{\mathfrak{k}}=\mathfrak{k} \oplus \mathbb{R} Z$ and $\xi \in \tilde{\mathfrak{g}}^{\star}$ is defined by $\langle\xi, Z\rangle=1$ and $\left.\xi\right|_{\mathfrak{g}}=0$. Accordingly, we set $\tilde{G}=\exp \{\tilde{\mathfrak{g}}\}$ and $\tilde{K}=\exp \{\tilde{\mathfrak{k}}\}$. We identify $\tilde{\mathfrak{g}}$ with $\tilde{G}$ via the global chart:

$$
a H+v_{1} \oplus v_{2}+t_{1} E \oplus t_{2} E+\ell Z \mapsto \exp \{a H\} \exp \left\{v_{1} \oplus v_{2}+t_{1} E \oplus t_{2} E+\ell Z\right\}
$$

where $a, t_{1}, t_{2}, \ell \in \mathbb{R}$ and $v_{1}, v_{2} \in V$. The group law of $\tilde{G}$ in these coordinates then reads:

$$
\begin{array}{r}
\left(a, v_{1}, v_{2}, t_{1}, t_{2}, \ell\right)\left(a^{\prime}, v_{1}^{\prime}, v_{2}^{\prime}, t_{1}^{\prime}, t_{2}^{\prime}, \ell^{\prime}\right)=\left(a+a^{\prime}, e^{-a^{\prime}} v_{1}+v_{1}^{\prime}, e^{a^{\prime}} v_{2}+v_{2}^{\prime},\right. \\
e^{-2 a^{\prime}} t_{1}+t_{1}^{\prime}+\frac{1}{2} e^{-a^{\prime}} \omega^{0}\left(v_{1}, v_{1}^{\prime}\right), e^{2 a^{\prime}} t_{2}+t_{2}^{\prime}+\frac{1}{2} e^{a^{\prime}} \omega^{0}\left(v_{2}, v_{2}^{\prime}\right), \\
\left.\ell+\ell^{\prime}+\left(e^{-2 a^{\prime}}-1\right) t_{1}+\left(e^{2 a^{\prime}}-1\right) t_{2}+\frac{1}{2} \omega^{0}\left(e^{-a^{\prime}} v_{1}-e^{a^{\prime}} v_{2}, v_{1}^{\prime}-v_{2}^{\prime}\right)\right)
\end{array}
$$

and the inversion map is given by:

$$
\begin{aligned}
& \left(a, v_{1}, v_{2}, t_{1}, t_{2}, \ell\right)^{-1}= \\
& \left(-a,-e^{a} v_{1},-e^{-a} v_{2},-e^{2 a} t_{1},-e^{-2 a} t_{2},-\ell-\left(e^{2 a}-1\right) t_{1}-\left(e^{-2 a}-1\right) t_{2}\right) .
\end{aligned}
$$

Moreover the involution $\tilde{\sigma}$ admits the following expression:

$$
\tilde{\sigma}\left(a, v_{1}, v_{2}, t_{1}, t_{2}, \ell\right)=\left(-a, v_{2}, v_{1}, t_{2}, t_{1}, \ell\right) .
$$

Under the parametrization of $\tilde{G}$ given above, we consider the following global coordinates system on $\tilde{G} / \tilde{K}$ :

$$
\tilde{G} / \tilde{K} \rightarrow \mathbb{R}^{2 d+2}, \quad\left(a, v_{1}, v_{2}, t_{1}, t_{2}, \ell\right) \widetilde{K} \mapsto\left(a, v_{1}-v_{2}, t_{1}-t_{2}-\frac{1}{2} \omega^{0}\left(v_{1}, v_{2}\right)\right)
$$

From the formula $s_{g \tilde{K}}\left(g^{\prime} \tilde{K}\right)=g \tilde{\sigma}\left(g^{-1} g^{\prime}\right) \tilde{K}$ for the symmetry on $\tilde{G} / \tilde{K}$, we deduce the following isomorphism of symplectic symmetric spaces:

Proposition 6.2. Under the identifications $\mathbb{S} \simeq \mathbb{R}^{2 d+2} \simeq \tilde{G} / \tilde{K}$ associated with the charts (2.4) and (6.7), the symplectic symmetric space $\tilde{G} / \tilde{K}$ underlying the exact siLa $(\tilde{\mathfrak{g}}, \tilde{\sigma}, \delta \xi)$ defined above, is isomorphic to the symplectic symmetric space $\left(\mathbb{S}, s, \omega^{\mathbb{S}}\right)$ underlying an elementary normal $\mathbf{j}$-group $\mathbb{S}$, as given in section $[2.1$.

Next, we need to endow $\left(\mathbb{S}, s, \omega^{\mathbb{S}}\right)$ with a structure of polarized symplectic symmetric space. From Lemma 5.9] it suffices to specify $W$, a k-invariant Lagrangian subspace of $\mathfrak{p}$. To this aim, we again consider the splitting of the $2 d$-dimensional symplectic vector space $\left(V, \omega^{0}\right)$ into a direct sum of two Lagrangian subspaces in symplectic duality:

$$
V=\mathfrak{l}^{\star} \oplus \mathfrak{l}
$$

Relatively to this decomposition and within the notation (6.3), we define:

$$
W:=\mathfrak{l}_{-} \oplus \mathbb{R} E_{-} \subset \mathfrak{g} .
$$

Following then Proposition 5.18, we let

$$
\mathfrak{b}:=\tilde{\mathfrak{k}} \oplus W=\mathfrak{k} \oplus \mathbb{R} Z \oplus W,
$$


be the polarization Lie algebra. Accordingly with the terminology introduced in Definition 5.19 we call $(\tilde{\mathfrak{g}}, \tilde{\sigma}, \xi, \mathfrak{b})$ the transvection quadruple of the symplectic symmetric space $\left(\mathbb{S}, s, \omega^{\mathbb{S}}\right)$.

Regarding the question of existence of $\tilde{G}$-invariant measures on the homogeneous spaces $\tilde{G} / \tilde{K}$ and $\tilde{G} / B$, we first observe the following fact:

Lemma 6.3. Let $(\mathfrak{g}=\mathfrak{k} \oplus \mathfrak{p}, \sigma)$ be the involutive Lie algebra associated to a solvable, simply connected, oriented symmetric space $M=G / K$ such that $[\mathfrak{p}, \mathfrak{p}]=\mathfrak{k}$. Then both $G$ and $K$ are unimodular Lie groups.

Proof. Under the orientation hypothesis, let $\nu_{\mathfrak{p}}$ denote a $K$-invariant volume element on $\mathfrak{p} \simeq T_{K} M$. Since $\mathfrak{k}=[\mathfrak{p}, \mathfrak{p}] \subset[\mathfrak{g}, \mathfrak{g}]$, under the solvability assumption, the Lie algebra $\mathfrak{k}$ is nilpotent. Therefore for every $Z \in \mathfrak{k}$, one has $\operatorname{Tr}\left(\left.\operatorname{ad}_{Z}\right|_{\mathfrak{k}}\right)=0$ and there exists an ad-invariant volume element $\nu_{\mathfrak{k}}$ on $\mathfrak{k}$. The volume element $\nu_{\mathfrak{k}} \wedge \nu_{\mathfrak{p}}$ on $\mathfrak{g}$ is therefore $\mathfrak{k}$-invariant. It is also ad $\mathfrak{d}_{\mathfrak{p}}$-invariant since, due to the iLa condition, for every $X \in \mathfrak{p}$, the element $\operatorname{ad}_{X}$ is trace-free. The simple-connectedness of $M$ implies the connectedness of $K$. The latter is therefore unimodular, as well as $G$.

Remark 6.4. The above lemma implies that $\tilde{G}, \tilde{K}$ and $B:=\exp \{\mathfrak{b}\}$ are all unimodular ( $\mathfrak{b}$ is nilpotent). In particular, there exist $\tilde{G}$-invariant measures on the homogeneous spaces $\tilde{G} / \tilde{K}$ and $\tilde{G} / B$.

Last, we need to specify the local and elementary structures underlying the polarized symplectic symmetric space $\left(\mathbb{S}, s, \omega^{\mathbb{S}}\right)$, as introduced in Definition 5.30 and Definition [5.35] respectively. We first note:

Lemma 6.5. Let

$$
\mathfrak{q}:=\mathfrak{a} \oplus\left(\mathfrak{l}^{\star} \oplus 0\right) \quad \text { and } \quad \mathfrak{Y}:=(\mathfrak{l} \oplus 0) \oplus \mathbb{R}((E \oplus 0)+Z) .
$$

Then $\mathfrak{q}$ is a Lie subalgebra of $\tilde{\mathfrak{g}}$ supplementary to $\mathfrak{b}$ and $\mathfrak{Y}$ is an Abelian Lie subalgebra of $\mathfrak{b}$ which is normalized by $\mathfrak{q}$. Moreover, the associated semi-direct product $\mathfrak{q} \ltimes \mathfrak{Y}$ is naturally isomorphic to the Lie algebra $\mathfrak{s}$ and induces the vector space decomposition $\tilde{\mathfrak{g}}=\mathfrak{s} \oplus \tilde{\mathfrak{k}}$.

Proof. First observe that for all $X \in \mathfrak{h}$, one has $X \oplus 0=X_{-}+X_{+} \in \mathfrak{h} \oplus \mathfrak{h}$ and therefore

$$
\begin{aligned}
\varpi(H, E \oplus 0) & =\varpi\left(H, E_{-}+E_{+}\right)=\varpi\left(H, E_{-}\right)=2, \\
\varpi\left(v \oplus 0, v^{\prime} \oplus 0\right) & =\varpi\left(v_{-}+v_{+}, v_{-}^{\prime}+v_{+}^{\prime}\right)=\varpi\left(v_{-}, v_{-}^{\prime}\right)=\omega^{0}\left(v, v^{\prime}\right), \quad \forall v, v^{\prime} \in V .
\end{aligned}
$$

The fact that $\mathfrak{q}$ is a Lie subalgebra follows from

$$
\left[H, \mathfrak{l}^{\star} \oplus 0\right]_{\tilde{\mathfrak{g}}}=\left[H, \mathfrak{l}^{\star} \oplus 0\right]+\varpi\left(H, \mathfrak{l}^{\star} \oplus 0\right) Z=\mathfrak{l}^{\star} \oplus 0,
$$

and

$$
\left[\mathfrak{l}^{\star} \oplus 0, \mathfrak{l}^{\star} \oplus 0\right]_{\tilde{\mathfrak{g}}}=\omega^{0}\left(\mathfrak{l}^{\star}, \mathfrak{l}^{\star}\right)(E \oplus 0+Z)=0 .
$$

Next, observe that $\mathfrak{Y}$ is Abelian:

$$
[\mathfrak{Y}, \mathfrak{Y}]_{\tilde{\mathfrak{g}}}=[\mathfrak{l} \oplus 0, \mathfrak{l} \oplus 0]_{\tilde{\mathfrak{g}}}+[\mathfrak{l} \oplus 0, \mathbb{R}((E \oplus 0)+Z)]_{\tilde{\mathfrak{g}}}=\omega^{0}(\mathfrak{l}, \mathfrak{l})((E \oplus 0)+Z)=0
$$

To see that $\mathfrak{q}$ normalizes $\mathfrak{Y}$, let $x \in \mathfrak{l}$ and $t \in \mathbb{R}$. Then one has

$$
\begin{aligned}
& {[H, x \oplus 0+t(E \oplus 0+Z)]_{\tilde{\mathfrak{g}}}} \\
& =[H, x \oplus 0]+\varpi\left(H, x_{-}\right) Z+t[H, E \oplus 0]+t \varpi\left(H, E_{-}\right) Z \\
& =x \oplus 0+2 t(E \oplus 0+Z) .
\end{aligned}
$$


Similarly, for all $y \in \mathfrak{l}^{\star}$, one has:

$[y \oplus 0, x \oplus 0+t(E \oplus 0+Z)]_{\tilde{\mathfrak{g}}}=[y \oplus 0, x \oplus 0]+\omega^{0}(y, x) Z=\omega^{0}(y, x)(E \oplus 0+Z)$.

The rest of the statement is immediate.

REMARK 6.6. Neither $\mathfrak{q}$ nor $\mathfrak{Y}$ are $\tilde{\sigma}$-stable. However, since $\tilde{\sigma}(\mathfrak{q})=\mathfrak{a} \oplus\left(0 \oplus \mathfrak{l}^{\star}\right)$ and $\left[0 \oplus \mathfrak{l}^{\star}, \mathfrak{Y}\right]=0$, one sees that $\tilde{\sigma}(\mathfrak{q})$ normalizes $\mathfrak{Y}$ as well.

Lemma 6.7. Equipped with the subgroup $Q=\exp \{\mathfrak{q}\}$ of $\tilde{G}$, the symplectic symmetric space $\left(\mathbb{S}, s, \omega^{\mathbb{S}}\right)$ is local in the sense of Definition 5.30.

Proof. Note first that

$\mathfrak{b}=\mathfrak{k} \oplus W \oplus \mathbb{R} Z=\mathfrak{h}_{+} \oplus \mathfrak{l}_{-} \oplus \mathbb{R} E_{-} \oplus \mathbb{R} Z=\mathfrak{l}_{+}^{\star} \oplus(\mathfrak{l} \oplus \mathfrak{l}) \oplus(\mathbb{R} E \oplus \mathbb{R} E) \oplus \mathbb{R} Z$.

Thus, under the parametrization (6.5) of $\tilde{G}$, we have

$$
B=\left\{\left(0, n \oplus m_{1}, n \oplus m_{2}, t_{1}, t_{2}, \ell\right): m_{1}, m_{2} \in \mathfrak{l}, n \in \mathfrak{l}^{\star}, t_{1}, t_{2}, \ell \in \mathbb{R}\right\},
$$

and

$$
Q=\left\{(a, n, 0,0,0,0): n \in \mathfrak{l}^{\star}, a \in \mathbb{R}\right\} .
$$

Thus for $q=(a, n, 0,0,0,0)$ and $b=\left(0, n^{\prime} \oplus m_{1}^{\prime}, n^{\prime} \oplus m_{2}^{\prime}, t_{1}^{\prime}, t_{2}^{\prime}, \ell^{\prime}\right) \in B$, we have using (6.6):

$$
q . b=\left(a,\left(n+n^{\prime}\right) \oplus m_{1}^{\prime}, n^{\prime} \oplus m_{2}^{\prime}, t_{1}^{\prime}+\frac{1}{2} \omega^{0}\left(n, m_{1}^{\prime}\right), t_{2}^{\prime}, \ell^{\prime}+\frac{1}{2} \omega^{0}\left(n, m_{1}^{\prime}-m_{2}^{\prime}\right)\right),
$$

from which we deduce that the map

$$
Q \times B \rightarrow \tilde{G}, \quad(q, b) \mapsto q . b,
$$

is a global diffeomorphism (i.e. the first condition of Definition 5.30 is satisfied). Note that identifying $B$ with $\mathfrak{b}$, one has

$$
b \tilde{\sigma}\left(b^{-1}\right)=2 b_{\mathfrak{p}}
$$

where we set $b=: b_{\mathfrak{\mathfrak { k }}}+b_{\mathfrak{p}}$ according to the vector space decomposition $\mathfrak{b}=\tilde{\mathfrak{k}} \oplus(\mathfrak{b} \cap \mathfrak{p})$.

For the second condition, observe that as $\mathfrak{b} \cap \mathfrak{p}=\mathfrak{l}_{-} \oplus \mathbb{R} E_{-}$, we get

$$
[\mathfrak{a}, \mathfrak{b} \cap \mathfrak{p}]=\left[\mathfrak{a}, \mathfrak{l}_{-}\right] \oplus\left[\mathfrak{a}, \mathbb{R} E_{-}\right]=\mathfrak{l}_{+} \oplus \mathbb{R} E_{+} \subset \mathfrak{h}_{+}=\mathfrak{k} \subset \mathfrak{b} .
$$

To check the last condition, consider $q=(a, n, 0,0,0,0) \in Q$, with $a \in \mathbb{R}, n \in \mathfrak{l}^{\star}$. We then have

$$
\tilde{\sigma} q=(-a, 0, n, 0,0,0)=(-a,-n, 0,0,0,0)(0, n, n, 0,0,0)=(\tilde{\sigma} q)^{Q}(\tilde{\sigma} q)^{B},
$$

since $(0, n, n, 0,0,0) \in \mathfrak{l}_{+}^{\star} \subset \mathfrak{h}_{+} \subset \mathfrak{b}$. Thus, $\chi\left((\tilde{\sigma} q)^{B}\right)=1$ since for $b=(0, n \oplus$ $\left.m_{1}, n \oplus m_{2}, t_{1}, t_{2}, \ell\right) \in B$, we have $\chi(g)=e^{i \ell}$.

Next, we come to the symmetric space structure of the group $Q$ :

LEMMA 6.8. In the global chart:

$$
\mathfrak{q} \simeq \mathfrak{a} \oplus \mathfrak{l}^{\star} \rightarrow Q, \quad(a, n) \mapsto \exp \{a H\} \exp \{n \oplus 0\},
$$

the left invariant symmetric space structure $\underline{s}$ on $Q$ described in Lemma 5.31 reads:

$$
\underline{s}_{(a, n)}\left(a^{\prime}, n^{\prime}\right)=\left(2 a-a^{\prime}, 2 \cosh \left(a-a^{\prime}\right) n-n^{\prime}\right) .
$$

Moreover, the symmetric space $(Q, \underline{s})$ admits a midpoint map, which in the coordinates above, is given by:

$$
\operatorname{mid}\left((a, n),\left(a_{0}, n_{0}\right)\right)=\left(\frac{a+a_{0}}{2}, \frac{n+n_{0}}{2} \operatorname{sech}\left(\frac{a-a_{0}}{2}\right)\right) .
$$


Proof. By definition we have $\underline{s}_{q} q^{\prime}=q \tilde{\sigma}\left(q^{-1} q^{\prime}\right)^{Q}$ and the formula for the symmetry follows easily from (6.6) and (6.9). The formula for the midpoint map comes from a direct computation of the inverse diffeomorphism of the partial map $\underline{s}^{q}:=\left[Q \ni q^{\prime} \mapsto \underline{s}_{q^{\prime}} q \in Q\right]$.

Remark 6.9. Setting $A:=\exp \{\mathfrak{a}\}$ and $N:=\exp \left\{\mathfrak{l}^{\star} \oplus 0\right\}$ we have the global decomposition $Q=A N$ and for $q=a n \in Q$, the symmetry at the neutral element reads $\underline{s}_{e} q=a^{-1} n^{-1}$. Also, the global chart

$$
\widetilde{G} / B \rightarrow \mathbb{R}^{d+1}, \quad\left(a, n_{1} \oplus m_{1}, n_{2} \oplus m_{2}, t_{1}, t_{2}, \ell\right) B \mapsto\left(a, n_{1}-n_{2}\right),
$$

$a, t_{1}, t_{2}, \ell \in \mathbb{R}, n_{1}, n_{2} \in \mathfrak{l}^{\star}, m_{1}, m_{2} \in \mathfrak{l}$, identifies $\tilde{G} / B$ with $Q$ via the coordinate system (6.10).

LEMma 6.10. The Abelian subgroup $\mathbb{Y}:=\exp \{\mathfrak{Y}\}$ of $\tilde{G}$, endows the local symplectic symmetric space $\left(\mathbb{S}, s, \omega^{\mathbb{S}}\right)$ with an elementary structure in the sense of Definition 5.35 .

Proof. We already know by Lemma 6.8, that the left invariant symmetric space $(Q, \underline{s})$ admits a midpoint map. We also know by Lemma 6.5 that $\mathbb{Y}$ is normalized by $Q$ and that $\mathbb{S}$ is isomorphic to $Q \ltimes \mathbb{Y}$. But we need to know that $Q \ltimes \mathbb{Y}$ acts simply transitively on the symmetric space $\tilde{G} / \tilde{K}$. For this, let $g=(a, v, 0, t, 0, t) \in Q \ltimes \mathbb{Y}$ and $g^{\prime}=\left(a^{\prime}, v_{1}^{\prime}, v_{2}^{\prime}, t_{1}^{\prime}, t_{2}^{\prime}, \ell^{\prime}\right) \in \tilde{G}$. Then we get

$$
\begin{aligned}
g g^{\prime}=\left(a+a^{\prime}, e^{-a^{\prime}} v+v_{1}^{\prime}, v_{2}^{\prime}, e^{-2 a^{\prime}} t+t_{1}^{\prime}+\frac{1}{2} e^{-a^{\prime}} \omega^{0}\left(v, v_{1}^{\prime}\right), t_{2}^{\prime},\right. \\
\left.\ell^{\prime}+e^{-2 a^{\prime}} t+\frac{1}{2} \omega^{0}\left(e^{-a^{\prime}} v, v_{1}^{\prime}-v_{2}^{\prime}\right)\right),
\end{aligned}
$$

and thus in the chart (6.7) of $\tilde{G} / \tilde{K}$, we get:

$g g^{\prime} \tilde{K} \mapsto\left(a+a^{\prime}, e^{-a^{\prime}} v+v_{1}^{\prime}-v_{2}^{\prime}, e^{-2 a^{\prime}} t+t_{1}^{\prime}-t_{2}^{\prime}-\frac{1}{2} \omega^{0}\left(v_{1}^{\prime}, v_{2}^{\prime}\right)+\frac{1}{2} \omega^{0}\left(e^{-a^{\prime}} v, v_{1}^{\prime}-v_{2}^{\prime}\right)\right)$.

This means that under the identification $\mathbb{S} \simeq \tilde{G} / \tilde{K}, Q \ltimes \mathbb{Y} \simeq \mathbb{S}$ acts by left translations and the second condition of Definition (5.35) is verified. For the third condition, note that under the parametrization (6.5) of $\tilde{G}$, we have

$$
\mathbb{Y}=\{(0, m, 0, t, 0, t): m \in \mathfrak{l}, t \in \mathbb{R}\} .
$$

Take $q=(a, n, 0,0,0,0) \in Q, a \in \mathbb{R}, n \in \mathfrak{l}^{\star}$ and $b=e^{y}=(0, m, 0, t, 0, t) \in \mathbb{Y}, m \in \mathfrak{l}$, $t \in \mathbb{R}$. A computation then shows that

$$
\left\langle\xi,\left(\operatorname{Ad}_{q^{-1}}-\operatorname{Ad}_{\left(\underline{s}_{e} q\right)^{-1}}\right) y\right\rangle=2 t \sinh 2 a+2 \cosh a \omega^{0}(n, m),
$$

which entails that

$$
\Psi(a, n)=(2 \sinh 2 a, 2 n \cosh a) .
$$

The last condition follows from (6.4).

Remark 6.11. Parametrizing $\tilde{G}$ as in (6.5), $\mathbb{S} \simeq \tilde{G} / \tilde{K}$ as in (6.7) and $Q \simeq \tilde{G} / B$ as in (6.12), we have the following expression for the action of $\tilde{G}$ on $\mathbb{S}$ :

$$
\begin{aligned}
& \left(a, v_{1}, v_{2}, t_{1}, t_{2}, \ell\right) \cdot\left(a^{\prime}, v^{\prime}, t^{\prime}\right) \\
& =\left(a+a^{\prime}, e^{-a^{\prime}} v_{1}-e^{a^{\prime}} v_{2}+v^{\prime}, e^{-2 a^{\prime}} t_{1}-e^{2 a^{\prime}} t_{2}+t^{\prime}-\frac{1}{2} \omega^{0}\left(v_{1}, v_{2}\right)\right),
\end{aligned}
$$

and on $Q$ :

$$
\left(a, n_{1} \oplus m_{1}, n_{2} \oplus m_{2}, t_{1}, t_{2}, \ell\right) \cdot\left(a^{\prime}, n^{\prime}\right)=\left(a+a^{\prime}, e^{-a^{\prime}} n_{1}-e^{a^{\prime}} n_{2}+n^{\prime}\right) .
$$


REMARK 6.12. From similar methods than those leading to Lemma 2.27, we deduce that we have:

$$
\mathfrak{d}_{Q} \asymp\left[(a, n) \in Q \mapsto \cosh a+|n|\left(1+e^{a}\right)\right] .
$$

From the Remark above and in analogy with Remark 2.36, we define the Fréchet valued Schwartz space of $Q$, denoted $\mathcal{S}(Q, \mathcal{E})$, as the set of smooth functions such that all left (or right) derivatives decrease faster than any power of $\mathfrak{d}_{Q}$. The latter space is Fréchet for the semi-norms:

$$
f \in \mathcal{S}(Q, \mathcal{E}) \mapsto \sup _{X \in \mathcal{U}_{k}(\mathfrak{q})} \sup _{x \in Q}\left\{\frac{\mathfrak{d}_{Q}(x)^{n}\|\tilde{X} f(x)\|_{j}}{|X|_{k}}\right\}, \quad j, k, n \in \mathbb{N},
$$

or even for

$$
f \in \mathcal{S}(Q, \mathcal{E}) \mapsto \sup _{X \in \mathcal{U}_{k}(\mathfrak{q})} \sup _{x \in Q}\left\{\frac{\mathfrak{d}_{Q}(x)^{n}\|\underline{X} f(x)\|_{j}}{|X|_{k}}\right\}, \quad j, k, n \in \mathbb{N} .
$$

\subsection{Quantization of elementary normal j-groups}

In this section, we specialize the different ingredients of our quantization map in the case of the elementary symplectic symmetric space $\left(\mathbb{S}, s, \omega^{\mathbb{S}}\right)$ determined by an elementary normal $\mathbf{j}$-group. We also (re)introduce a real parameter $\theta$ in the definition of the character (5.7):

$$
\chi_{\theta}(b):=\exp \left\{\frac{i}{\theta}\langle\xi, \log (b)\rangle\right\}, \quad b \in B, \quad \theta \in \mathbb{R}^{*},
$$

which is globally defined as $B$ is exponential. By Lemma 5.17 and Remark 6.4, the Haar measure $\mathrm{d}_{\mathbb{S}}$ on $\mathbb{S}\left(\right.$ respectively $\mathrm{d}_{Q}$ on $Q$ ) is invariant under both $s_{e}^{\star}$ (respectively $\underline{s}_{e}^{\star}$ ) and $\tilde{G}$. Observe that under the parametrization (6.8) of the group $B$, we have $\chi_{\theta}(b)=\exp \left\{\frac{i}{\theta} \ell\right\}$. Note that within the chart (6.10), any left-invariant Haar measure $\mathrm{d}_{Q}$ on $Q$ is a multiple of the Lebesgue measure on $\mathfrak{q}$ (these facts are transparent in Equations (2.5), (6.11) and in Remark 6.11). Also, within the chart (2.4), any left invariant Haar measure $d_{\mathbb{S}}$ on $\mathbb{S}$ is a multiple of the Lebesgue measure on $\mathfrak{q} \ltimes \mathfrak{Y}$. By Remark 5.36 (iii), the restriction to $\mathbb{S}=Q \ltimes \mathbb{Y}$ of the induced representation $U_{\chi_{\theta}}$ (that we denote by $U_{\theta}$ from now on) of $\tilde{G}$ on $L^{2}\left(Q, \mathrm{~d}_{Q}\right)$ reads within the charts (2.4) on $\mathbb{S}$ and (6.10) on $Q$ :

$$
\begin{aligned}
& U_{\theta}(a, v, t) \psi\left(a_{0}, n_{0}\right) \\
& =\exp \left\{\frac{i}{\theta}\left(e^{2\left(a-a_{0}\right)} t+\omega^{0}\left(\frac{1}{2} e^{a-a_{0}} n-n_{0}, e^{a-a_{0}} m\right)\right)\right\} \psi\left(a_{0}-a, n_{0}-e^{a-a_{0}} n\right),
\end{aligned}
$$

where $(a, v, t) \in \mathbb{S}$ with $a, t \in \mathbb{R}$ and $v=n \oplus m \in \mathfrak{l}^{\star} \oplus \mathfrak{l}=V$ and $\left(a_{0}, n_{0}\right) \in Q$ with $a_{0} \in \mathbb{R}$ and $n_{0} \in \mathfrak{l}^{\star}$.

REMARK 6.13. In accordance with the notations of earlier chapters, from now on, we make explicit the dependence on the parameter $\theta \in \mathbb{R}^{*}$ in all the objects we are considering. For instance, we now set $\Omega_{\theta, \mathbf{m}}$ instead $\Omega_{\mathbf{m}}$ for the quantization map, $\star_{\theta, \mathbf{m}}$ instead of $\star_{\mathbf{m}}$ for the associated composition product, $K_{\theta, \mathbf{m}}$ instead of $K_{\mathbf{m}}$ for its three-points kernel, $\mathbf{E}_{\theta}$ instead of $\mathbf{E}$ for the one-point phase etc.

Lemma 6.14. Within the coordinates (2.4) on $\mathbb{S}$ and under the decomposition $v=n \oplus m \in \mathfrak{l}^{\star} \oplus \mathfrak{l}=V$, the one-point phase $\mathbf{E}_{\theta}$ of Lemma 5.32] reads

$$
\mathbf{E}_{\theta}(a, v, t)=\exp \left\{-\frac{2 i}{\theta}\left(t \sinh 2 a+\omega^{0}(n, m) \cosh ^{2} a\right)\right\} .
$$


Proof. Recall that from Lemma 5.37, we have:

$$
\mathbf{E}_{\theta}\left(q^{-1} e^{y}\right)=\exp \left\{\frac{i}{\theta}\langle\xi,[\Psi(q), y]\rangle\right\} .
$$

Which from (6.13) becomes:

$$
\mathbf{E}_{\theta}\left(q^{-1} e^{y}\right)=\exp \left\{\frac{2 i}{\theta}\left(t \sinh 2 a+\omega^{0}(n, m) \cosh a\right)\right\},
$$

with $q=(a, n, 0,0,0,0) \in Q, a \in \mathbb{R}, n \in \mathfrak{l}^{\star}$ and $b=e^{y}=(0, m, 0, t, 0, t) \in \mathbb{Y}, m \in \mathfrak{l}$, $t \in \mathbb{R}$. Since $q^{-1}=\left(-a,-e^{a} n, 0,0,0,0\right)$, one gets

$$
\mathbf{E}_{\theta}(q b)=\exp \left\{-\frac{2 i}{\theta}\left(t \sinh 2 a+\omega^{0}(n, m) e^{a} \cosh a\right)\right\} .
$$

One concludes by observing that the coordinates $q b$ are related to the coordinates (2.4) through

$$
(a, v, t)=(a, n, 0,0,0,0) .\left(0, m, 0, t-\frac{1}{2} \omega^{0}(n, m), 0, t-\frac{1}{2} \omega^{0}(n, m)\right) .
$$

From (6.11) and (6.13), we observe:

$$
\left|\operatorname{Jac}_{\underline{s}^{e}}(a, n)\right|=2^{d+1} \cosh ^{d} a, \quad\left|\operatorname{Jac}_{\Psi}(a, n)\right|=2^{d+2} \cosh 2 a \cosh ^{d} a,
$$

so that the element $\mathbf{m}_{0}$ given in (5.12) reads:

$$
\mathbf{m}_{0}(a, n)=2^{d+2} \cosh ^{1 / 2} 2 a \cosh ^{d} a .
$$

From this, we deduce:

Proposition 6.15. Parametrizing $\mathbb{S}$ as in (2.4) and $Q$ as in (6.10), we have the following expression for the action on $L^{2}\left(Q, \mathrm{~d}_{Q}\right)$ of the unitary quantizer $\Omega_{\theta, \mathbf{m}_{0}}(x)$, $x \in \mathbb{S}$, associated with the polarized symplectic symmetric space underlying an elementary normal $\mathbf{j}$-group $\mathbb{S}$ :

$$
\begin{aligned}
& \Omega_{\theta, \mathbf{m}_{0}}(a, v, t) \psi\left(a_{0}, n_{0}\right)=2^{d+1} \cosh \left(2 a-2 a_{0}\right)^{1 / 2} \cosh \left(a-a_{0}\right)^{d} \\
& \times \exp \left\{\frac{2 i}{\theta}\left(\sinh \left(2 a-2 a_{0}\right) t+\omega^{0}\left(\cosh \left(a-a_{0}\right) n-n_{0}, \cosh \left(a-a_{0}\right) m\right)\right)\right\} \\
& \times \psi\left(2 a-a_{0}, 2 \cosh \left(a-a_{0}\right) n-n_{0}\right) .
\end{aligned}
$$

REMARK 6.16. Observe that $\left|\mathrm{Jac}_{\underline{s}}\right|$ is $\underline{s}_{e}$-invariant, as it is an even function of the variable $a$ only. Thus, by Lemma 5.28 and Remark 5.42, we deduce that the unitary quantization map $\Omega_{\theta, \mathbf{m}_{0}}$ is also compatible with the natural involutions of its source and range spaces (the complex conjugation on $L^{2}\left(\mathbb{S}, \mathrm{d}_{\mathbb{S}}\right)$ and the adjoint on $\mathcal{L}^{2}\left(L^{2}\left(Q, \mathrm{~d}_{Q}\right)\right)$. In particular, it sends real-valued functions to self-adjoint operators.

Our next result is one of the key steps of this chapter: it renders transparent the link between chapters 3 and 4 and chapters 5 and 6 . For this, we need the explicit expression of the tri-kernel $K_{\theta, \mathbf{m}_{\mathbf{0}}}$ of the product (5.14) for an elementary normal jgroup $\mathbb{S}$. First, observe that the unique solution of the equation $\underline{s}_{q_{2}} \circ \underline{s}_{q_{1}} \circ \underline{s}_{q_{0}}(q)=q$, $q, q_{0}, q_{1}, q_{2} \in Q$, as given in Lemma 5.53, reads

$$
q=\left(a_{0}-a_{1}+a_{2}, \cosh \left(a_{0}-a_{1}\right) n_{2}-\cosh \left(a_{2}-a_{0}\right) n_{1}+\cosh \left(a_{1}-a_{2}\right) n_{0}\right) .
$$

From [6, 23, we extract

Lemma 6.17. Within the notations of Proposition 5.55, we have

$$
\mathcal{J}=\left|\operatorname{Jac}_{\Phi_{Q}}\right|
$$


Then, Proposition 5.55 and a straightforward computation gives $K_{\theta, \mathbf{m}_{\mathbf{0}}}=$ $A_{\mathbf{m}_{\mathbf{0}}} e^{\frac{2 i}{\theta} S}$ with:

$$
\begin{aligned}
A_{\mathbf{m}_{\mathbf{0}}}\left(x_{1}, x_{2}, x_{3}\right)= & \mathbf{m}_{\mathbf{0}}\left(a_{1}-a_{2}\right) \mathbf{m}_{\mathbf{0}}\left(a_{2}-a_{3}\right) \mathbf{m}_{\mathbf{0}}\left(a_{3}-a_{1}\right) \\
= & 2^{3 d+3} \cosh \left(2 a_{1}-2 a_{2}\right)^{1 / 2} \cosh \left(a_{1}-a_{2}\right)^{d} \cosh \left(2 a_{2}-2 a_{3}\right)^{1 / 2} \\
& \quad \times \cosh \left(a_{2}-a_{3}\right)^{d} \cosh \left(2 a_{3}-2 a_{1}\right)^{1 / 2} \cosh \left(a_{3}-a_{1}\right)^{d},
\end{aligned}
$$

and

$$
\begin{aligned}
S\left(x_{1}, x_{2}, x_{3}\right)= & \sinh \left(2 a_{2}-2 a_{1}\right) t_{3}+\sinh \left(2 a_{1}-2 a_{3}\right) t_{2}+\sinh \left(2 a_{3}-2 a_{2}\right) t_{1} \\
& +\cosh \left(a_{1}-a_{2}\right) \cosh \left(a_{2}-a_{3}\right) \omega^{0}\left(v_{3}, v_{1}\right) \\
& +\cosh \left(a_{2}-a_{3}\right) \cosh \left(a_{3}-a_{1}\right) \omega^{0}\left(v_{1}, v_{2}\right) \\
& +\cosh \left(a_{3}-a_{1}\right) \cosh \left(a_{1}-a_{2}\right) \omega^{0}\left(v_{2}, v_{3}\right) .
\end{aligned}
$$

By identification, we thereby obtain:

Proposition 6.18. For $g_{1}, g_{2}, g_{3} \in \mathbb{S}$ and $\theta \in \mathbb{R}^{*}$, we have

$$
K_{\theta, \mathbf{m}_{0}}\left(g_{1}, g_{2}, g_{3}\right)=K_{\theta, 0}\left(g_{1}^{-1} g_{2}, g_{1}^{-1} g_{3}\right),
$$

where the three-point kernel on the left hand side of the above equality is given in Proposition 5.55 and the two-point kernel on the right hand side is given in Theorem 3.5 for $\tau=0$. In particular, the products $\star_{\theta, \mathbf{m}_{0}}$ and $\star_{\theta, 0}$ coincide on $L^{2}\left(\mathbb{S}, \mathrm{d}_{\mathbb{S}}\right)$.

Before giving the link between the generic kernels $K_{\theta, \mathbf{m}}$ and $K_{\theta, \tau}$, hence a fortiori between the generic products $\star_{\theta, \mathbf{m}}$ and $\star_{\theta, \tau}$, we will give the relation between our quantization map and Weyl's. Denote by $\Omega^{0}$, the Weyl quantization map of $\mathbb{S}$ in the Darboux chart (2.4). For a function $f$ on $\mathbb{S}, \Omega^{0}(f)$ is an operator on $L^{2}\left(\mathbb{R}^{d+1}\right) \simeq L^{2}\left(Q, \mathrm{~d}_{Q}\right)$ given (up to a normalization constant) by

$$
\begin{aligned}
& \Omega^{0}(f) \psi\left(a_{0}, n_{0}\right):= \\
& C(\theta, d) \int_{\mathbb{R}^{2 d+2}} e^{-\frac{i}{\theta}\left(2\left(a_{0}-a\right) t+\omega^{0}\left(n_{0}-n, m\right)\right)} f\left(\frac{a+a_{0}}{2}, \frac{n+n_{0}}{2}, m, t\right) \psi(a, n) \mathrm{d} a \mathrm{~d} n \mathrm{~d} m \mathrm{~d} t .
\end{aligned}
$$

Then, recall that for $\tau \in \boldsymbol{\Theta}$ (see Definition 3.2), the inverse $T_{\theta, \tau}^{-1}$ of the map (3.1) is continuous on the 'flat' Schwartz space $1 \mathcal{S}(\mathbb{S})$. As the Weyl quantization maps continuously Schwartz functions to trace-class operators, we deduce that $\Omega^{0} \circ T_{\theta, \tau}^{-1}$ is well defined and continuous from $\mathcal{S}(\mathbb{S})$ to $\mathcal{L}^{1}\left(L^{2}\left(Q, \mathrm{~d}_{Q}\right)\right)$. From this, we get

Proposition 6.19. Let $\tau \in \Theta$ (see Definition 3.2). Then, as continuous operators from $2^{2} \mathcal{S}(\mathbb{S})$ to the trace ideal $\mathcal{L}^{1}\left(L^{2}\left(Q, \mathrm{~d}_{Q}\right)\right)$, we have

$$
\Omega^{0} \circ T_{\theta, \tau}^{-1}=\Omega_{\theta, \mathbf{m}}, \quad \text { where } \mathbf{m}(a, n)=\mathbf{m}_{0}(a, n) \exp \left\{\tau\left(\frac{2}{\theta} \sinh 2 a\right)\right\} .
$$

ProOF. By density, it suffices to show that for $f \in \mathcal{S}(\mathbb{S})$, the operators $\Omega_{\theta, \mathbf{m}}(f)$ and $\Omega^{0}\left(T_{\theta, \tau}^{*}(f)\right)$ coincide on $\mathcal{D}(Q)$. Note then that for $f \in \mathcal{S}(\mathbb{S})$, we have

$$
\begin{aligned}
& T_{\theta, \tau}^{-1}(f)(a, v, t)= \\
& 2 \pi \int_{\mathbb{R}^{2}} \frac{\cosh ^{1 / 2}\left(\frac{\theta \xi}{2}\right)}{\cosh ^{d}\left(\frac{\theta \xi}{4}\right)} e^{\tau\left(\frac{2}{\theta} \sinh \left(\frac{\theta \xi}{2}\right)\right)} f\left(a, \operatorname{sech}\left(\frac{\theta \xi}{4}\right) v, t^{\prime}\right) e^{i \xi t-i \frac{2}{\theta} \sinh \left(\frac{\theta \xi}{2}\right) t^{\prime}} \mathrm{d} \xi \mathrm{dt}^{\prime} .
\end{aligned}
$$

\footnotetext{
${ }^{1}$ By this we mean the ordinary Schwartz space in the global chart (2.4).

${ }^{2}$ Observe that $\mathcal{S}^{S_{\text {can }}}(\mathbb{S}) \subset \mathcal{S}(\mathbb{S})$.
} 
Hence, for $\psi \in \mathcal{D}(Q)$, we get

$$
\begin{aligned}
& \Omega^{0}\left(T_{\theta, \tau}^{-1}(f)\right) \psi\left(a_{0}, n_{0}\right)= \\
& C_{1}(d) \int_{\mathbb{R}^{2 d+4}} \frac{\cosh ^{1 / 2}\left(\frac{\theta \xi}{2}\right)}{\cosh ^{d}\left(\frac{\theta \xi}{4}\right)} e^{\tau\left(\frac{2}{\theta} \sinh \left(\frac{\theta \xi}{2}\right)\right)} e^{-\frac{i}{\theta}\left(2\left(a_{0}-a\right) t+\omega^{0}\left(n_{0}-n, m\right)\right)} e^{i \xi t-i \frac{2}{\theta} \sinh \left(\frac{\theta \xi}{2}\right) t^{\prime}} \\
& \times f\left(\frac{a+a_{0}}{2}, \operatorname{sech}\left(\frac{\theta \xi}{4}\right)\left(n+n_{0}\right) / 2, \operatorname{sech}\left(\frac{\theta \xi}{4}\right) m, t^{\prime}\right) \psi(a, n) \mathrm{d} \xi \mathrm{dt}^{\prime} \mathrm{d} a \mathrm{~d} n \mathrm{~d} m \mathrm{~d} t .
\end{aligned}
$$

Performing the change of variable $a \mapsto 2 a-a_{0}, n \mapsto 2 \cosh \left(\frac{\theta \xi}{4}\right) n-n_{0}$ and $m \mapsto$ $\cosh \left(\frac{\theta \xi}{4}\right) m$, we get

$$
\begin{aligned}
& \Omega^{0}\left(T_{\theta, \tau}^{-1}(f)\right) \psi\left(a_{0}, n_{0}\right)= \\
& C_{2}(d) \int_{\mathbb{R}^{2 d+4}} \cosh ^{1 / 2}\left(\frac{\theta \xi}{2}\right) \cosh ^{d}\left(\frac{\theta \xi}{4}\right) e^{\tau\left(\frac{2}{\theta} \sinh \left(\frac{\theta \xi}{2}\right)\right)} f\left(a, n, m, t^{\prime}\right) e^{i \xi t-i \frac{2}{\theta} \sinh \left(\frac{\theta \xi}{2}\right) t^{\prime}} \\
& \times e^{-\frac{2 i}{\theta}\left(2\left(a_{0}-a\right) t+\omega^{0}\left(n_{0}-\cosh \left(\frac{\theta \xi}{4}\right) n, \cosh \left(\frac{\theta \xi}{4}\right) m\right)\right)} \psi\left(2 a-a_{0}, 2 \cosh \left(\frac{\theta \xi}{4}\right) n-n_{0}\right) \\
& \times \mathrm{d} \xi \mathrm{dt}^{\prime} \mathrm{d} a \mathrm{~d} n \mathrm{~d} m \mathrm{~d} t .
\end{aligned}
$$

Integrating out the $t$-variable yields a factor $\delta\left(\xi-\frac{4}{\theta}\left(a_{0}-a\right)\right)$ and thus we get

$$
\begin{aligned}
& \Omega^{0}\left(T_{\theta, \tau}^{-1}(f)\right) \psi\left(a_{0}, n_{0}\right)= \\
& C_{3}(d) \int_{\mathbb{R}^{2 d+2}} \cosh ^{1 / 2} 2\left(a-a_{0}\right) \cosh ^{d}\left(a-a_{0}\right) e^{\tau\left(\frac{2}{\theta} \sinh \left(2\left(a-a_{0}\right)\right)\right)} f\left(a, n, m, t^{\prime}\right) \\
& \times e^{\frac{2 i}{\theta}\left(\sinh \left(2\left(a-a_{0}\right)\right) t^{\prime}+\omega^{0}\left(\cosh \left(a-a_{0}\right) n-n_{0}, \cosh \left(a-a_{0}\right) m\right)\right)} \\
& \times \psi\left(2 a-a_{0}, 2 \cosh \left(a-a_{0}\right) n-n_{0}\right) \mathrm{d} a \mathrm{~d} n \mathrm{~d} m \mathrm{~d} t^{\prime},
\end{aligned}
$$

which by Proposition 6.15 coincides with $\Omega_{\theta, \mathbf{m}}(f) \psi$.

From the above result and the defining relation (3.2) for the product $\star_{\theta, \tau}$ for a generic element $\tau \in \boldsymbol{\Theta}$, we then deduce:

Proposition 6.20. To every $\tau \in \boldsymbol{\Theta}$, associate a right- $N$-invarian 3 function $\mathbf{m}$ on $Q$ as in (6.14). Then, the three point kernel $K_{\theta, \mathbf{m}}$ of the product $\star_{\theta, \mathbf{m}}$ defined in Proposition 5.52 (ii), is related to the two-point kernel $K_{\theta, \tau}$ given in Theorem 3.5 via:

$$
K_{\theta, \mathbf{m}}\left(g_{1}, g_{2}, g_{3}\right)=K_{\theta, \tau}\left(g_{1}^{-1} g_{2}, g_{1}^{-1} g_{2}\right), \quad \forall g_{1}, g_{2}, g_{3} \in \mathbb{S}
$$

In particular, the product $\star_{\theta, \mathbf{m}}$ is well defined on $\mathcal{B}(\mathbb{S})$ and coincide with $\star_{\theta, \tau}$.

REMARK 6.21. From now on, to indicate that a right- $N$-invariant borelian function $\mathbf{m}$ on $Q$ is associated to an element $\tau \in \boldsymbol{\Theta}$, as in (6.14), we just write $\mathbf{m} \in \boldsymbol{\Theta}(\mathbb{S})$.

REMARK 6.22. Observe that, considering the 'medial triangle' three-point function $\Phi_{\mathbb{S}}$ given in Proposition 2.14 (ii), and writing $K_{\theta, \mathbf{m}}=A_{\mathbf{m}} e^{\frac{2 i}{\theta} S}$, for $\mathbf{m}$ a right$N$-invariant function on $Q$, we have:

$$
\begin{aligned}
A_{\mathbf{m}}\left(x_{1}, x_{2}, x_{3}\right) & =\mathbf{m}\left(a_{1}-a_{2}\right) \frac{\mathbf{m}_{0}^{2}\left(a_{2}-a_{3}\right)}{\overline{\mathbf{m}}\left(a_{2}-a_{3}\right)} \mathbf{m}\left(a_{3}-a_{1}\right) \\
& =\left|\mathrm{Jac}_{\Phi_{\mathbb{S}}^{-1}}\left(x_{1}, x_{2}, x_{3}\right)\right|^{1 / 2} \frac{\mathbf{m}}{\mathbf{m}_{0}}\left(a_{1}-a_{2}\right) \frac{\mathbf{m}_{0}}{\overline{\mathbf{m}}}\left(a_{2}-a_{3}\right) \frac{\mathbf{m}}{\mathbf{m}_{0}}\left(a_{3}-a_{1}\right) .
\end{aligned}
$$

\footnotetext{
${ }^{3}$ See Remark 6.9 for the definition of the abelian subgroup $N$.
} 
The expression above for the amplitude (in the case where $\mathbf{m}$ is right- $N$-invariant), could also be derived from the explicit expression for the Berezin transform (see Definition [5.46). Indeed, in the present situation, its distributional kernel in coordinates (2.4), reads:

$$
\begin{aligned}
& B_{\theta, \mathbf{m}}\left[x_{1}, x_{2}\right] \\
& =\delta\left(a_{1}-a_{2}\right) \delta\left(n_{1}-n_{2}\right) \delta\left(m_{1}-m_{2}\right) \int_{\mathbb{R}} \frac{|\mathbf{m}|^{2}}{\mathbf{m}_{0}^{2}}\left(\frac{1}{2} \operatorname{arcsinh}\left(\frac{1}{2} a_{0}\right)\right) e^{\frac{i \theta}{2} a_{0}\left(t_{1}-t_{2}\right)} \mathrm{d} a_{0} .
\end{aligned}
$$

By standard Fourier-analysis arguments, we deduce:

$$
B_{\theta, \mathbf{m}}=\frac{|\mathbf{m}|^{2}}{\mathbf{m}_{\mathbf{0}}^{2}}\left(\frac{1}{2} \operatorname{arcsinh}\left(\frac{i}{\theta} \partial_{t}\right)\right) .
$$

Finally, let us discuss the question of the involution for the generic product $\star_{\theta, \mathbf{m}}$. Since in general, the formal adjoint of $\Omega_{\theta, \mathbf{m}}(x)$ on $L^{2}\left(Q, \mathrm{~d}_{Q}\right)$ is $\Omega_{\theta, \underline{s}_{e}^{\star}} \overline{\mathbf{m}}(x)$, we deduce

$$
\overline{f_{1} \star_{\theta, \mathbf{m}} f_{2}}=\bar{f}_{2} \star_{\theta, \underline{s}_{e}^{\star}} \overline{\mathbf{m}} \overline{f_{1}} .
$$

Hence, we obtain that the natural involution for the product $\star_{\theta, \mathbf{m}}$ is

$$
*_{\theta, \mathbf{m}}: f \mapsto \frac{\mathbf{m}}{\underline{s}_{e}^{\star} \overline{\mathbf{m}}}\left(\frac{1}{2} \operatorname{arcsinh}\left(\frac{i}{\theta} \partial_{t}\right)\right) \bar{f} .
$$

REMARK 6.23. From Theorem 5.43 and the previous expression for the involution, we observe that the element $\mathbf{m}_{0}$ defined in (5.12) is uniquely determined by the requirement that the associated quantization map is both unitary and involution preserving.

\subsection{Quantization of normal j-groups}

Consider now a normal $\mathbf{j}$-group $\mathbb{B}=\left(\mathbb{S}_{N} \ltimes_{\mathbf{R}^{N-1}} \ldots\right) \ltimes_{\mathbf{R}^{1}} \mathbb{S}_{1}$ with associated extension morphisms

$$
\mathbf{R}^{j} \in \operatorname{Hom}\left(\left(\mathbb{S}_{N} \ltimes \ldots\right) \ltimes \mathbb{S}_{j+1}, \operatorname{Sp}\left(V_{j}, \omega_{j}^{0}\right)\right), \quad j=1, \ldots, N-1,
$$

as in (2.3). We wish to apply Proposition 5.62 to this situation. For this, recall that for $\mathbb{S}$ an elementary normal $\mathbf{j}$-group viewed as an elementary symplectic symmetric space, $\mathfrak{D}$ denotes the Lie algebra of $W$-preserving symplectic endomorphisms of $\mathfrak{p}$ where the Lagrangian subspace $W$ has been chosen to be $\mathfrak{l}_{-} \oplus \mathbb{R} E_{-} \simeq \mathfrak{l} \oplus \mathbb{R} E$.

Proposition 6.24. Denote by $\mathfrak{D}_{0}$ the stabilizer Lie subalgebra in $\mathfrak{s p}\left(V, \omega^{0}\right)$ of the Lagrangian subspace $\mathfrak{l}$.

(i) Let $\operatorname{Sym}(\mathfrak{l})$ be the space of endomorphisms of $\mathfrak{l}$ that are symmetric with respect to a given Euclidean scalar product on $\mathfrak{l}$. Let also

$$
\eta: \operatorname{End}(\mathfrak{l}) \times \operatorname{Sym}(\mathfrak{l}) \rightarrow \operatorname{Sym}(\mathfrak{l}), \quad(T, S) \mapsto T \circ S+S \circ T^{t} .
$$

Then, endowing $\operatorname{Sym}(\mathfrak{l})$ with the structure of an Abelian Lie algebra, one has the isomorphism:

$$
\mathfrak{D}_{0} \simeq \operatorname{End}(\mathfrak{l}) \ltimes_{\eta} \operatorname{Sym}(\mathfrak{l}) .
$$

(ii) The Lie algebra $\mathfrak{D}_{0}$ contains an Iwasawa component of $\mathfrak{s p}\left(V, \omega^{0}\right)$.

(iii) Letting $\mathfrak{D}_{0}$ trivially act on the central element $E$ of $\mathfrak{h}$ induces an isomorphism:

$$
\mathfrak{D} \simeq \mathfrak{D}_{0} \ltimes \mathfrak{h}
$$


ProOF. Item (i) is immediate from an investigation at the matrix form level.

Item (iii) follows from the fact that the derivation algebra of the non-exact polarized transvection symplectic triple $(\mathfrak{g}, \sigma, \varpi)$ underlying an elementary normal j-group $\mathbb{S}$ admits the symplectic Lie algebra $\mathfrak{s p}\left(V, \omega^{0}\right)$ as Levi-factor $\mathbf{3}$.

Item (ii) follows from a dimensional argument combined with Borel's conjugacy Theorem of maximal solvable subgroups in complex simple Lie groups. Indeed, on the first hand, the dimension of the Iwasawa factor of $\mathfrak{s p}\left(V, \omega^{0}\right)$ equals $\operatorname{dim} \mathfrak{s p}\left(V, \omega^{0}\right)-\operatorname{dim} \mathfrak{u}(d)$ that is $2 d+\frac{2 d(2 d-1)}{2}-d^{2}=d(d+1)$ with $2 d:=\operatorname{dim} V=$ $2 \operatorname{dim} \mathfrak{l}$. On the other hand, the dimension of the Borel factor in $\operatorname{End}(\mathfrak{l})$ equals $d+\frac{d(d-1)}{2}$ which equals $\operatorname{dim} \operatorname{Sym}(\mathfrak{l})$. Hence $\mathfrak{D}_{0}$ contains a maximal solvable Lie subalgebra of dimension $2\left(d+\frac{d(d-1)}{2}\right)=d(d+1)$. Borel's Theorem then yields the assertion since $\mathfrak{s p}\left(V, \omega^{0}\right)$ is totally split.

From [3], we observe that the full polarization quadruple of $\mathbb{S}$ underlies the Lie group $\mathbb{L}=\operatorname{Sp}\left(V, \omega^{0}\right) \ltimes \tilde{G}$. Hence:

COROllary 6.25. Let $\mathbb{S}$ be an elementary normal $\mathbf{j}$-group viewed as an elementary symplectic symmetric space (see Definition 5.35 ) and let $\left(\mathbb{L}, \sigma_{\mathbb{L}}, \xi, \mathbf{B}\right)$ be the associated full polarization quadruple (see Definition 5.19). Then we have the global decomposition $\mathbb{L}=Q \mathbf{B}$ and moreover $\left.\Delta_{\mathbb{L}}\right|_{\mathbf{B}}=\Delta_{\mathbf{B}}$.

We can now prove the conditions needed to apply Proposition 5.62

Proposition 6.26. Let $\mathbb{B}=\left(\mathbb{S}_{N} \ltimes_{\mathbf{R}^{N-1}} \ldots\right) \ltimes_{\mathbf{R}^{1}} \mathbb{S}_{1}$ be a normal $\mathbf{j}$-group, to which one associates the full polarization quadruples $\left(\mathbb{L}_{j}, \sigma_{\mathbb{L}_{j}}, \xi_{j}, \mathbf{B}_{j}\right), j=1, \ldots, N$, of the $\mathbb{S}_{j}$ 's. Then, there exists an homomorphism $\rho_{j}:\left(\mathbb{S}_{N} \ltimes \ldots\right) \ltimes \mathbb{S}_{j} \rightarrow \tilde{\mathbf{D}}_{j-1}$ whose image normalizes $\mathbb{S}_{j-1}$ in $\mathbb{L}_{j-1}$ and such that the extension homomorphism $\mathbf{R}_{j}$ constructed in (5.16), coincides with the extension homomorphism $\mathbf{R}^{j}$ underlying the Pyatetskii-Shapiro's decomposition (6.16).

Proof. Firstly, by Pyatetskii-Shapiro's theory [22], one knows that the action of $\left(\mathbb{S}_{N} \ltimes \ldots\right) \ltimes \mathbb{S}_{j}$ on $\mathbb{S}_{j-1}$ factors through a solvable subgroup of $\operatorname{Sp}\left(V_{j-1}, \omega_{j-1}^{0}\right)$. Setting $(A N)_{j-1}$ the Iwasawa factor of $\operatorname{Sp}\left(V_{j-1}, \omega_{j-1}^{0}\right)$, we thus get an homomorphism:

$$
\tilde{\rho}_{j}:\left(\mathbb{S}_{N} \ltimes \ldots\right) \ltimes \mathbb{S}_{j} \rightarrow(A N)_{j-1} .
$$

But Proposition 6.24 (ii) asserts that $(A N)_{j-1}$ is a subgroup of $\exp \left\{\mathfrak{D}_{0, j-1}\right\}$, where $\mathfrak{D}_{0, j-1}$ is the stabilizer Lie subalgebra in $\mathfrak{s p}\left(V_{j-1}, \omega_{j-1}^{0}\right)$ of the Lagrangian subspace $\mathfrak{l}_{j-1}$. Combining this with the isomorphism of Proposition 6.24 (iii), yields another homomorphism:

$$
\hat{\rho}_{j}:(A N)_{j-1} \rightarrow \mathbf{D}_{j-1} \subset \tilde{\mathbf{D}}_{j-1} .
$$

Hence $\rho_{j}:=\hat{\rho}_{j} \circ \tilde{\rho}_{j}$ is the desired homomorphism. Now, observe that by $\mathbf{3}$. Proposition 2.2 item (i)], the group $\mathbb{S}_{j-1}$ viewed as a subgroup of $\mathbb{L}_{j-1}$, is normalized by $\operatorname{Sp}\left(V_{j-1}, \omega_{j-1}^{0}\right)$ for the action given in Proposition 2.9 (iv) and that this action is precisely the one associated with the extension homomorphism $\mathbf{R}^{j}$ in the decomposition (6.16). Thus, all that remains to do is to prove that the extension homomorphisms $\mathbf{R}^{j}$ and $\mathbf{R}_{j}$ coincide. Here, $\mathbf{R}_{j}(g):=\mathbf{C}_{\rho_{j}(g)} \in \operatorname{Aut}\left(\mathbb{S}_{j-1}\right), g \in \mathbb{S}_{j}$, is the extension homomorphism constructed in (5.16). But that $\mathbf{R}^{j}=\mathbf{R}_{j}$ follows from a very general fact about homogeneous spaces. Namely, observe that if $M=G / K$, then action of the isotropy $K \times M \rightarrow M,(k, g K) \rightarrow k g K$, lifts to $G$ as 
the restriction to $K$ of the conjugacy action $K \times G \rightarrow G,(k, g) \mapsto k g k^{-1}$ (indeed: $\left.k g K=k g k^{-1} K\right)$.

From this, we deduce that Proposition 5.62 and Theorem 5.63 are valid in the case of a normal $\mathbf{j}$-group. Moreover, we also deduce that the associated product $\star_{\theta, \mathbf{m}}$ coincides with $\star_{\theta, \vec{\tau}}$ of Proposition 3.7

Proposition 6.27. Let $\mathbb{B}$ be a normal $\mathbf{j}$-group. To every $\vec{\tau} \in \mathbf{\Theta}^{N}$, we associate a function $\mathbf{m}$ on $Q_{N} \times \ldots Q_{1}$ by $\mathbf{m}=\mathbf{m}_{N} \otimes \cdots \otimes \mathbf{m}_{1}$ where $\mathbf{m}_{j}$ is related to $\tau_{j}$ as in (6.14). Then, the three-point kernel $K_{\theta, \mathbf{m}}$ of the product $\star_{\theta, \mathbf{m}}$ defined in Theorem 5.63 (iii), is related with the two-point kernel $K_{\theta, \tau}$ given in Proposition 3.7 (3.5) via:

$$
K_{\theta, \mathbf{m}}\left(g_{1}, g_{2}, g_{3}\right)=K_{\theta, \vec{\tau}}\left(g_{1}^{-1} g_{2}, g_{1}^{-1} g_{2}\right), \quad \forall g_{1}, g_{2}, g_{3} \in \mathbb{B} .
$$

In particular, the product $\star_{\theta, \mathbf{m}}$ is well defined on $\mathcal{B}(\mathbb{B})$ and coincide with $\star_{\theta, \vec{\tau}}$.

REMARK 6.28. To indicate that a function $\mathbf{m}=\mathbf{m}_{N} \otimes \cdots \otimes \mathbf{m}_{1}$ on $Q_{N} \times \ldots Q_{1}$ is related to elements $\tau_{j} \in \boldsymbol{\Theta}$ as in (6.14), we just write $\mathbf{m} \in \mathbf{\Theta}(\mathbb{B})$.

We also quote the following extension of Proposition 6.19,

Proposition 6.29. Let $\mathbb{B}$ be a normal $\mathbf{j}$-group. For any $\mathbf{m} \in \mathbf{\Theta}(\mathbb{B})$, the quantization map $\Omega_{\theta, \mathbf{m}}$ is a continuous operator from $\mathcal{S}(\mathbb{B}):=\mathcal{S}\left(\mathbb{S}_{N}\right) \otimes \cdots \otimes \mathcal{S}\left(\mathbb{S}_{1}\right)$ to $\mathcal{L}^{1}\left(L^{2}\left(Q_{N}, \mathrm{~d}_{Q_{N}}\right) \otimes \cdots \otimes L^{2}\left(Q_{1}, \mathrm{~d}_{Q_{1}}\right)\right)$.

Our last result concerns the representation homomorphism (5.17).

Lemma 6.30. Let $\mathbb{B}=\mathbb{B}^{\prime} \ltimes_{\mathbf{R}} \mathbb{S}_{1}, \mathbb{S}_{1}=Q_{1} \ltimes \mathbb{Y}_{1}$. Then, the restriction of the homomorphism $\mathcal{R}: \mathbb{B}^{\prime} \rightarrow \mathcal{U}\left(L^{2}\left(Q_{1}\right)\right.$ ) (see (5.17)) underlying the one $\rho=: \mathbb{B}^{\prime} \rightarrow \tilde{\mathbf{D}}_{1}$ of Proposition 6.26, defines a tempered action (see Definition 4.1) of $\mathbb{B}^{\prime}$ on $\mathcal{S}\left(Q_{1}\right)$.

Proof. Parametrizing $\mathbb{S}_{1} \simeq \mathfrak{s}_{1}=\{(a, n, m, t)\}, Q_{1} \simeq q_{1}=\{(a, n)\}$ as usual, the matrix of the Pyatetskii-Shapiro extension homomorphism $\mathbf{R}_{g^{\prime}}$ is expressed under the form

$$
\mathbf{R}_{g^{\prime}}=\left(\begin{array}{cccc}
0 & 0 & 0 & 0 \\
0 & \mathbf{R}_{+}\left(g^{\prime}\right) & 0 & 0 \\
0 & \mathbf{R}_{-}\left(g^{\prime}\right) & \left(\mathbf{R}_{+}\left(g^{\prime}\right)^{T}\right)^{-1} & 0 \\
0 & 0 & 0 & 0
\end{array}\right),
$$

where (by Borel's conjugacy theorem) the element $\mathbf{R}_{+}\left(g^{\prime}\right)$ is (conjugated to) an upper triangular matrix form in End $\left(\mathfrak{l}^{\star}\right)$. The determinant of this element then consists (as a small induction argument shows) in a product of powers of the modular functions of the elementary factors of $\mathbb{S}_{2}, \ldots, \mathbb{S}_{N}$ of $\mathbb{B}^{\prime}$ :

$$
\operatorname{det}\left(\mathbf{R}_{+}\right)=\Pi_{j=2}^{N} \Delta_{\mathbb{S}_{j}}^{n_{j}}
$$

One deduces from a careful examination of the construction of $\rho$ in Proposition 6.26, that $\mathcal{R}$ is explicitly given by:

$$
\mathcal{R}\left(g^{\prime}\right) \varphi(a, n)=\operatorname{det}\left(\mathbf{R}_{+}\left(g^{\prime}\right)\right)^{-\frac{1}{2}} \varphi\left(a, \mathbf{R}_{+}\left(g^{\prime}\right)^{-1} n\right) .
$$

Temperedeness results in a direct computation using that the semi-norms of $\mathcal{S}\left(Q_{1}\right)$ are given by (see the discussion right after Remark [6.12):

$$
\|f\|_{k, n}:=\sup _{X \in \mathcal{U}_{k}\left(\mathfrak{q}_{1}\right)} \sup _{x \in Q_{1}}\left\{\frac{\mathfrak{d}_{Q_{1}}(x)^{n}|\tilde{X} f(x)|_{j}}{|X|_{k}}\right\},
$$


that left invariant vector fields associated with $H$ the generator of $\mathfrak{a}$ and $\left\{f_{j}\right\}_{j=1}^{d}$ be a basis of $\mathfrak{l}^{\star}$, read in the coordinates (6.10):

$$
\widetilde{H}=\partial_{a}-\sum_{j=1}^{d} n_{j} \partial_{n_{j}}, \quad \widetilde{f}_{j}=\partial_{n_{j}}, \quad j=1, \ldots, d,
$$

together with the behavior of the modular weight $\mathfrak{d}_{Q_{1}}$ given in Remark 6.12). 



\section{CHAPTER 7}

\section{Deformation of $C^{*}$-algebras}

Throughout this chapter, we consider a $C^{*}$-algebra $A$, endowed with an isometric and strongly continuous action $\alpha$ of a normal $\mathbf{j}$-group $\mathbb{B}$. In chapter 4 we have seen how to deform the Fréchet algebra $A^{\infty}$, consisting of smooth vectors for the action $\alpha$. Our goal here is to construct a $C^{*}$-norm on $\left(A^{\infty}, \star_{\theta, \mathbf{m}}^{\alpha}\right)$, in order to get, after completion, a deformation theory at the $C^{*}$-level. We stress that from now on, the isometricity assumption of the action is fundamental. The way we will define this $C^{*}$-norm is based on the pseudo-differential calculus introduced in the previous two chapters.

The basic ideas of the construction can be summarized as follow. Consider $\mathcal{H}$, a separable Hilbert space carrying a faithful representation of $A$. We will thereof identify $A$ with its image in $\mathcal{B}(\mathcal{H})$. Let $\mathcal{S}^{S_{\text {can }}^{\mathbb{B}}}(\mathbb{B}, A)$ be the $A$-valued one-point Schwartz space associated to the tempered pair $\left(\mathbb{B} \times \mathbb{B}, S_{\text {can }}^{\mathbb{B}}\right)$ as given in Definition 1.40. Since this space is a subset of the flat $A$-valued Schwartz space of $\mathbb{B}$, Proposition 6.29 shows that for every $\mathbf{m} \in \mathbf{\Theta}(\mathbb{B})$, the map

$$
f \in \mathcal{S}^{S_{\mathrm{can}}^{\mathbb{B}}}(\mathbb{B}, A) \mapsto \Omega_{\theta, \mathbf{m}}^{\mathbb{B}}(f):=\int_{\mathbb{B}} \Omega_{\theta, \mathbf{m}}^{\mathbb{B}}(x) \otimes f(x) \mathrm{d}_{\mathbb{B}}(x),
$$

is well defined and takes value 1 in $\mathcal{K}\left(\mathcal{H}_{\chi}\right) \otimes A$, where $\mathcal{H}_{\chi}:=L^{2}\left(Q_{1}\right) \otimes \cdots \otimes L^{2}\left(Q_{N}\right)$. Then, the main step is to extend the map (7.1), from $\mathcal{S}^{S_{\text {can }}^{\mathbb{B}}}(\mathbb{B}, A)$ to $\mathcal{B}(\mathbb{B}, A)$. As for $a \in A^{\infty}$, the $A$-valued function $\alpha(a):=\left[g \in \mathbb{B} \mapsto \alpha_{g}(a) \in A\right]$ belongs to $\mathcal{B}(\mathbb{B}, A)$, we will define a new norm on $A^{\infty}$ by setting

$$
\|a\|_{\theta, \mathbf{m}}:=\left\|\Omega_{\theta, \mathbf{m}}^{\mathbb{B}}(\alpha(a))\right\|,
$$

where the norm on the right hand side above, denotes the $C^{*}$-norm of $\mathcal{B}\left(\mathcal{H}_{\chi} \otimes \mathcal{H}\right)$. This will eventually be achieved by proving a non-Abelian $C^{*}$-valued version of the Calderón-Vaillancourt Theorem in the context of the present pseudo-differential calculus. This theorem will be proved using wavelet analysis and oscillatory integrals methods.

\subsection{Wavelet analysis}

Let $\mathbb{B}$ be a normal $\mathbf{j}$-group, with Pyatetskii-Shapiro decomposition

$$
\mathbb{B}=\left(\mathbb{S}_{N} \ltimes \ldots\right) \ltimes \mathbb{S}_{1},
$$

where the $\mathbb{S}_{j}$ 's, $j=1, \ldots, N$, are elementary normal $\mathbf{j}$-groups. Recall that our choice of parametrization is:

$$
\mathbb{S}_{N} \times \cdots \times \mathbb{S}_{1} \rightarrow \mathbb{B}, \quad\left(g_{N}, \ldots, g_{1}\right) \mapsto g_{1} \ldots g_{N} .
$$

\footnotetext{
${ }^{1}$ Given a Hilbert space $\mathcal{H}, \mathcal{K}(\mathcal{H})$ denotes the $C^{*}$-algebra of compact operators.
} 
REMARK 7.1. Observe that the extension homomorphism at each step, $\mathbf{R}^{j}$, being valued in $\operatorname{Sp}\left(V_{j}, \omega_{j}^{0}\right)$, it preserves any left invariant Haar measure $\mathrm{d}_{\mathbb{S}_{j}}$ on $\mathbb{S}_{j}$ :

$$
\left(\mathbf{R}_{g^{\prime}}^{j}\right)^{\star} \mathrm{d}_{\mathbb{S}_{j}}=\mathrm{d}_{\mathbb{S}_{j}}, \quad \forall g^{\prime} \in\left(\mathbb{S}_{N} \ltimes \ldots\right) \ltimes \mathbb{S}_{j-1} .
$$

This implies that the product of left invariant Haar measures $\mathrm{d}_{\mathbb{S}_{1}} \otimes \cdots \otimes \mathrm{d}_{\mathbb{S}_{N}}$ defines a left invariant Haar measure on $\mathbb{B}$ under both parametrizations $g=g_{1} \ldots g_{N}$ or $g=g_{N} \ldots g_{1}$ of $g \in \mathbb{B}$.

The aim of this section is to construct a weak resolution of the identity on the tensor product Hilbert space $L^{2}\left(Q_{N}\right) \otimes \cdots \otimes L^{2}\left(Q_{1}\right):=\mathcal{H}_{\chi}$ from a suitable family of coherent states for $\mathbb{B}$, that we now introduce.

Definition 7.2. Let $\mathbb{B}$ be a normal j-group. Given a mother wavelet $\eta \in$ $\mathcal{D}\left(Q_{N} \times \cdots \times Q_{1}\right)$, let $\left\{\eta_{x}\right\}_{x \in \mathbb{B}}$ be the family of coherent states defined by

$$
\eta_{x}:=U_{\theta}(x) \eta, \quad x \in \mathbb{B},
$$

where $U_{\theta}$ is the unitary representation of $\mathbb{B}$ on $\mathcal{H}_{\chi}$ constructed in Lemma 5.59 for the morphism underlying Proposition 6.26.

Observe that in the elementary case, we have:

$$
\eta_{x}\left(q_{0}\right)=\mathbf{E}_{\theta}^{0}\left(q^{-1} q_{0} b\right) \eta\left(q^{-1} q_{0}\right), \quad x=q b \in \mathbb{S}, \quad q_{0} \in Q,
$$

where the phase $\mathbf{E}_{\theta}^{0}$ is defined by

$$
\mathbf{E}_{\theta}^{0}(x):=\chi_{\theta}\left(\mathbf{C}_{q^{-1}}(b)\right), \quad x=q b \in \mathbb{S} .
$$

In the generic case, setting $\mathbb{B}=\mathbb{B}^{\prime} \ltimes_{\mathbf{R}} \mathbb{S}_{1}$ with $\mathbb{B}^{\prime}$ a normal $\mathbf{j}$-group and $\mathbb{S}_{1}$ an elementary normal $\mathbf{j}$-group, for $\eta=\eta^{\prime} \otimes \eta^{1}, \eta^{\prime} \in \mathcal{D}\left(Q_{N} \times \cdots \times Q_{2}\right), \eta^{1} \in \mathcal{D}\left(Q_{1}\right)$ and parametrizing $g \in \mathbb{B}$ as $g=g^{\prime} . g_{1}, g^{\prime} \in \mathbb{B}^{\prime}, g_{1} \in \mathbb{S}_{1}$, we have (see Lemma [5.59):

$$
\eta_{g}=\eta_{g^{\prime}}^{\prime} \otimes \mathcal{R}\left(g^{\prime}\right) \eta_{g_{1}}^{1},
$$

where $\mathcal{R}: \mathbb{B}^{\prime} \rightarrow U\left(L^{2}\left(Q_{1}\right)\right)$ is the homomorphism underlying (5.17). Parametrizing now $g \in \mathbb{B}$ as $g=g_{1} . g^{\prime}, g^{\prime} \in \mathbb{B}^{\prime}, g_{1} \in \mathbb{S}_{1}$, we find

$$
\eta_{g}=\eta_{g^{\prime}}^{\prime} \otimes\left(\mathcal{R}\left(g^{\prime}\right) \eta^{1}\right)_{g_{1}},
$$

Proposition 7.3. Let $\mathbb{B}$ be a normal $\mathbf{j}$-group, $\mathcal{E}$ a complex Fréchet space and $\eta \in \mathcal{D}\left(Q_{N} \times \cdots \times Q_{1}\right)$. Then, the map

$$
\begin{aligned}
& \mathcal{F}^{\eta}: L^{\infty}\left(Q_{N} \times \cdots \times Q_{1}, \mathcal{E}\right) \rightarrow L^{\infty}(\mathbb{B}, \mathcal{E}), \\
& f \mapsto\left[x \in \mathbb{B} \mapsto \int_{Q_{1} \times \cdots \times Q_{N}} f\left(q_{N}, \ldots, q_{1}\right) \eta_{x}\left(q_{N}, \ldots, q_{1}\right) \mathrm{d}_{Q_{N}}\left(q_{N}\right) \ldots \mathrm{d}_{Q_{1}}\left(q_{1}\right) \in \mathcal{E}\right],
\end{aligned}
$$

restricts as a continuous map $\mathcal{F}^{\eta}: \mathcal{S}\left(Q_{N} \times \cdots \times Q_{1}, \mathcal{E}\right) \rightarrow \mathcal{S}^{S_{\text {can }}}(\mathbb{B}, \mathcal{E})$.

Proof. Assume first that $\mathbb{B}=\mathbb{S}$ is an elementary normal $\mathbf{j}$-group. We denote by $\mathbf{E}_{\theta}^{0}$ the element of $C^{\infty}(\mathbb{S})$ given in (7.4), so that with $x=q b \in \mathbb{S}, q \in Q, b \in \mathbb{Y}$, we have for every $f \in \mathcal{S}(Q, \mathcal{E})$ :

$$
\left(\mathcal{F}^{\eta} f\right)(x)=\int_{Q} f\left(q_{0}\right) \mathbf{E}_{\theta}^{0}\left(q^{-1} q_{0} b\right) \eta\left(q^{-1} q_{0}\right) \mathrm{d}_{Q}\left(q_{0}\right)=\int_{Q} f\left(q q_{0}\right) \mathbf{E}_{\theta}^{0}\left(q_{0} b\right) \eta\left(q_{0}\right) \mathrm{d}_{Q}\left(q_{0}\right) .
$$

Decomposing as usual $\mathfrak{q}=\mathfrak{a} \oplus \mathfrak{n}$, we let $H$ be the generator of $\mathfrak{a}$ and $\left\{f_{j}\right\}_{j=1}^{d}$ be a basis of $\mathfrak{n}$. Then, from the expressions given in (2.8), we see that the associated left invariant vector fields read in the coordinates (6.10): 


$$
\widetilde{H}=\partial_{a}-\sum_{j=1}^{d} n_{j} \partial_{n_{j}}, \quad \widetilde{f}_{j}=\partial_{n_{j}}, \quad j=1, \ldots, d .
$$

Moreover, in the chart (2.4) of $\mathbb{S}$, with $x=(a, n \oplus m, t)$, the function $\mathbf{E}_{\theta}^{0}$ takes the following form:

$$
\mathbf{E}_{\theta}^{0}(x)=\exp \left\{\frac{i}{\theta}\left(e^{-2 a} t-e^{-a} \omega^{0}(n, m)\right)\right\} .
$$

Hence, defining

$$
i \widetilde{H} \mathbf{E}_{\theta}^{0}=: \alpha \mathbf{E}_{\theta}^{0} \quad \text { and } \quad-\sum_{j=1}^{d} \widetilde{f}_{j}^{2} \mathbf{E}_{\theta}^{0}=: \beta \mathbf{E}_{\theta}^{0},
$$

a simple computation gives

$$
\alpha(x)=\frac{2}{\theta}\left(e^{-2 a} t-e^{-a} \omega^{0}(n, m)\right), \quad \beta(x)=\theta^{-2} e^{-2 a}|m|^{2},
$$

where $|m|^{2}=\sum_{j=1}^{d} \omega^{0}\left(f_{j}, m\right)^{2}$. Moreover, it is easy to see that both $\alpha$ and $\beta$ are eigenvectors of $\widetilde{H}$ with eigenvalue -2 and that $\widetilde{f}_{j} \beta=0$. Hence setting $\widetilde{P}:=$ $1-\sum_{j=1}^{d} \widetilde{f}_{j}^{2}$, we get by integration by parts on the $q_{0}$-variable and with $k, k^{\prime} \in \mathbb{N}$ arbitrary:

$$
\begin{aligned}
& \left(\mathcal{F}^{\eta} f\right)(x)= \\
& \int_{Q} \mathbf{E}_{\theta}^{0}\left(q_{0} b\right)\left(1-\widetilde{H}_{q_{0}}^{2 k^{\prime}}\right)\left[\frac{\widetilde{P}_{q_{0}}^{k}\left[f\left(q q_{0}\right) \eta\left(q_{0}\right)\right]}{\left(1+\alpha\left(q_{0} b\right)^{2 k^{\prime}}+\alpha^{\left(k^{\prime}\right)}\left(q_{0} b\right)\right)\left(1+\beta\left(q_{0} b\right)\right)^{k}}\right] \mathrm{d}{ }_{Q}\left(q_{0}\right)
\end{aligned}
$$

where

$$
\alpha^{\left(k^{\prime}\right)}:=\sum_{r=1}^{2 k^{\prime}-1} c_{r}^{k^{\prime}} \alpha^{r} \quad\left(c_{r}^{k^{\prime}} \in \mathbb{C}\right)
$$

This easily entails that

$$
\left\|\left(\mathcal{F}^{\eta} f\right)(x)\right\|_{j} \leq C\left(k, k^{\prime}\right) \int_{Q} \frac{\left\|\left(1-\widetilde{H}_{q_{0}}^{2 k^{\prime}}\right) \widetilde{P}_{q_{0}}^{k}\left[f\left(q q_{0}\right) \eta\left(q_{0}\right)\right]\right\|_{j}}{\left(1+\alpha\left(q_{0} b\right)^{2 k^{\prime}}\right)\left(1+\beta\left(q_{0} b\right)\right)^{k}} \mathrm{~d}_{Q}\left(q_{0}\right) .
$$

By left invariance of $\widetilde{H}$ and $\widetilde{P}$, we get up to a redefinition of $f \in \mathcal{S}(Q, \mathcal{E})$ and of $\eta \in \mathcal{D}(Q)$ :

$$
\left\|\left(\mathcal{F}^{\eta} f\right)(x)\right\|_{j} \leq C\left(k, k^{\prime}\right) \int_{Q} \frac{\left\|f\left(q q_{0}\right)\right\|_{j}\left|\eta\left(q_{0}\right)\right|}{\left(1+\alpha\left(q_{0} b\right)^{2 k^{\prime}}\right)\left(1+\beta\left(q_{0} b\right)\right)^{k}} \mathrm{~d}_{Q}\left(q_{0}\right) .
$$

Now, given any tempered weight $\mu$ on $\mathbb{S}$, one therefore has

$$
\begin{aligned}
& \int_{\mathbb{S}} \mu(x)\left\|\left(\mathcal{F}^{\eta} f\right)(x)\right\|_{j} \mathrm{~d}_{\mathbb{S}}(x) \leq \\
& \quad C\left(k, k^{\prime}\right) \int_{Q^{2} \times \mathbb{Y}} \frac{\mu(q b)\left\|f\left(q q_{0}\right)\right\|_{j}\left|\eta\left(q_{0}\right)\right|}{\left(1+\alpha\left(q_{0} b\right)^{2 k^{\prime}}\right)\left(1+\beta\left(q_{0} b\right)\right)^{k}} \mathrm{~d}_{Q}\left(q_{0}\right) \mathrm{d}_{Q}(q) \mathrm{d}_{\mathbb{Y}}(b) .
\end{aligned}
$$

Observing that for every $X \in \mathcal{U}(\mathfrak{q})$, the element $\widetilde{X}_{q}\left[q b \mapsto \mathbf{E}_{\theta}^{0}(q b)\right]$ only depends on the variable $\mathbf{C}_{q^{-1}}(b)=$ : $\exp \operatorname{Ad}_{q^{-1}} y$, where $b=e^{y} \in \mathbb{Y}$, changing the variable 
following $b_{0}:=: \exp \left(y_{0}\right):=\mathbf{C}_{q_{0}^{-1}}(b)\left(y_{0} \in \mathfrak{Y}\right)$ yields

$$
\begin{aligned}
& \int_{Q^{2} \times \mathbb{Y}} \frac{\mu(q b)\left\|f\left(q q_{0}\right)\right\|_{j}\left|\eta\left(q_{0}\right)\right|}{\left(1+\alpha\left(q_{0} b\right)^{2 k^{\prime}}\right)\left(1+\beta\left(q_{0} b\right)\right)^{k}} \mathrm{~d}_{Q}\left(q_{0}\right) \mathrm{d}_{Q}(q) \mathrm{d}_{\mathbb{Y}}(b)= \\
& \int_{Q^{2} \times \mathbb{Y}} \frac{\mu\left(q q_{0} b_{0} q_{0}^{-1}\right)\left|\operatorname{det} \operatorname{Ad}_{q_{0}}\right| \mathfrak{Y}\left|\left\|f\left(q q_{0}\right)\right\|_{j}\right| \eta\left(q_{0}\right) \mid}{\left(1+\alpha\left(b_{0}\right)^{2 k^{\prime}}\right)\left(1+\beta\left(b_{0}\right)\right)^{k}} \mathrm{~d}_{Q}\left(q_{0}\right) \mathrm{d}_{Q}(q) \mathrm{d}_{Q}\left(b_{0}\right) .
\end{aligned}
$$

Writing $y_{0}=\left(m_{0}, t_{0}\right) \in \mathfrak{l} \times \mathbb{R}$ as before, we observe that $\alpha\left(b_{0}\right)=\frac{2 t_{0}}{\theta}, \beta\left(b_{0}\right)=$ $\theta^{-2}\left|m_{0}\right|^{2}$ and $\mathrm{d}_{\mathbb{Y}}\left(b_{0}\right)=\mathrm{d} y_{0}$. Therefore this last integral exists as soon as $k$ and $k^{\prime}$ are large enough in front of the polynomial growth of $\mu$. Since for every $Y \in \mathcal{U}(\mathfrak{s})$, one has $\widetilde{Y}_{x}\left[x \mapsto \eta_{x}\right]=\left(d U_{\theta}(Y) \eta\right)_{x}$, the left-invariant derivatives of $\mathcal{F}^{\eta} f$ follow the exact same treatment.

Passing to non-elementary case, we first observe that by Lemma 6.30, the restricted action $\mathcal{R}$ of the tempered Lie group $\mathbb{B}^{\prime}$ on $\mathcal{S}\left(Q_{1}\right)$ is tempered in the sense of Definition 4.1. Therefore, in the above discussion, replacing $\eta$ by $\mathcal{R}\left(g^{\prime}\right) \eta$ yields constants $C\left(k, k^{\prime}\right)$ that now depend polynomially on the tempered weights $\left\{\mu_{k, j}^{\mathcal{R}} \in C^{\infty}\left(\mathbb{B}^{\prime}\right)\right\}$ associated to the tempered action $\mathcal{R}$ (within the notations of Definition 4.1).

Now, within the conventions of (7.5) and considering $f:=f^{\prime} \otimes f_{1} \in \mathcal{S}\left(Q_{N} \times \ldots \times\right.$ $\left.Q_{2}, \mathcal{E}\right) \otimes \mathcal{S}\left(Q_{1}\right)$ and $\eta:=\eta^{\prime} \otimes \eta^{1} \in \mathcal{D}\left(Q_{N} \times \ldots \times Q_{2}\right) \otimes \mathcal{D}\left(Q_{1}\right)$, one has:

$$
\left(\mathcal{F}^{\eta} f\right)\left(g_{1} g^{\prime}\right)=\left(\mathcal{F}^{\eta^{\prime}} f^{\prime}\right)\left(g^{\prime}\right)\left(\mathcal{F}^{\mathcal{R}\left(g^{\prime}\right) \eta^{1}} f_{1}\right)\left(g_{1}\right) \text {. }
$$

Let $d \mathcal{R}$ the infinitesimal form of $\mathcal{R}$, that is for $\eta^{1} \in \mathcal{D}\left(Q_{1}\right)$, it is defined by $d \mathcal{R}(X) \eta^{1}:=\left.\frac{d}{d t}\right|_{t=0} \mathcal{R}\left(e^{t X}\right) \eta^{1}$ for $X \in \mathfrak{b}^{\prime}$ and extended as an algebra morphism to $\mathcal{U}\left(\mathfrak{b}^{\prime}\right)$. Note that for any $X \in \mathcal{U}\left(\mathfrak{b}^{\prime}\right)$ and $g^{\prime} \in \mathbb{B}^{\prime}$, we have

$$
\widetilde{X}_{g^{\prime}} \mathcal{R}\left(g^{\prime}\right) \eta^{1}=\mathcal{R}\left(g^{\prime}\right)\left(d \mathcal{R}(X) \eta^{1}\right) .
$$

Now, for every tempered weight $\mu=\mu^{\prime} \otimes \mu_{1}$ on $\mathbb{B}$ and $X=X^{\prime} \otimes X_{1} \in \mathcal{U}(\mathfrak{b})$ (obvious notations), denoting

$$
\tilde{\eta}^{1}:=d \mathcal{R}\left(X_{(2)}^{\prime}\right) \eta^{1} \in \mathcal{D}\left(Q_{N} \times \cdots \times Q_{2}\right),
$$

we then have

$$
\begin{array}{r}
\leq \sum_{\left(X^{\prime}\right)} \int_{\mathbb{B}^{\prime}} \mu^{\prime}\left(g^{\prime}\right) \int_{\mathbb{S}_{1}} \mu_{1}\left(g_{1}\right)\left\|\widetilde{X_{(1)}^{\prime} g^{\prime}}\left(\mathcal{F}^{\eta^{\prime}} f^{\prime}\left(g^{\prime}\right)\right) \widetilde{\left.X_{(2)}^{\prime}\right)_{g^{\prime}}} \widetilde{X_{1 g_{1}}}\left(\mathcal{F}^{\mathcal{R}\left(g^{\prime}\right) \eta^{1}} f_{1}\left(g_{1}\right)\right)\right\|_{j} \mathrm{~d} g_{1} \mathrm{~d} g^{\prime} \\
=\sum_{\left(X^{\prime}\right)} \int_{\mathbb{B}^{\prime}} \mu^{\prime}\left(g^{\prime}\right)\left\|\widetilde{X_{(1)}^{\prime} g_{g^{\prime}}}\left(\mathcal{F}^{\eta^{\prime}} f^{\prime}\left(g^{\prime}\right)\right)\right\|_{j} \int_{\mathbb{S}_{1}} \mu_{1}\left(g_{1}\right)\left|\widetilde{X_{1 g_{1}}}\left(\mathcal{F}^{\left.\mathcal{R}\left(g^{\prime}\right) \tilde{\eta}^{1}\right)} f_{1}\left(g_{1}\right)\right)\right| \mathrm{d} g_{1} \mathrm{~d} g^{\prime}
\end{array}
$$

From what we have proven in the elementary case and by induction hypothesis on $N$, the map

$$
\mathbb{B}^{\prime} \rightarrow \mathbb{R}_{+}, \quad g^{\prime} \mapsto \int_{\mathbb{S}_{1}} \mu_{1}\left(g_{1}\right)\left|\widetilde{X_{1 g_{1}}}\left(\mathcal{F}^{\left.\mathcal{R}\left(g^{\prime}\right) \tilde{\eta}^{1}\right)} f_{1}\left(g_{1}\right)\right)\right| \mathrm{d} g_{1},
$$

is temperate. Hence, the integral (7.7) exists. We conclude by nuclearity of the Schwartz spaces $\mathcal{S}\left(Q_{N} \times \cdots \times Q_{1}, \mathcal{E}\right)$.

We then deduce the following consequence: 
COROLlary 7.4. Let $\mathbb{B}$ be a normal $\mathbf{j}$-group and $\eta \in \mathcal{D}\left(Q_{N} \times \cdots \times Q_{1}\right)$. Then, the maps $\left[\mathbb{B} \ni x \mapsto\left\langle\eta, \eta_{x}\right\rangle\right]$ and $\left[\mathbb{B} \ni x \mapsto\left\langle\eta, \eta_{x^{-1}}\right\rangle\right]$ belong to $L^{1}(\mathbb{B})$.

Proof. This follows from Proposition 7.3 with $\mathcal{E}=\mathbb{C}$ since $\left\langle\eta, \eta_{x}\right\rangle=\mathcal{F}^{\bar{\eta}}(\eta)(x)$ and $\left\langle\eta, \eta_{x^{-1}}\right\rangle=\overline{\left\langle\eta, \eta_{x}\right\rangle}$.

The next result is probably well known 3 but since we are unable to locate it in the literature and since we use it several times, we deliver a proof.

Lemma 7.5. Let $(X, \mu)$ be a $\sigma$-finite measure space and $\mathcal{H}$ a separable Hilbert space. Consider an element

$$
K \in L^{\infty}(X \times X, \mu \otimes \mu ; \mathcal{B}(\mathcal{H})),
$$

such that

$c_{1}^{2}:=\sup _{x \in X} \int_{X}\|K(x, y)\|_{\mathcal{B}(\mathcal{H})} d \mu(y)<\infty, \quad c_{2}^{2}:=\sup _{y \in X} \int_{X}\|K(x, y)\|_{\mathcal{B}(\mathcal{H})} d \mu(x)<\infty$.

Then, the associated kernel operator is bounded on $L^{2}(X, \mu ; \mathcal{H}) \simeq L^{2}(X, \mu) \otimes \mathcal{H}$ with operator norm not exceeding $c_{1} c_{2}$.

Proof. Let $T_{K}$ be the operator associated with the kernel $K$. For vectors $\Phi, \Psi \in L^{2}(X, \mu ; \mathcal{H}) \cap L^{1}(X, \mu ; \mathcal{H})$, we have $\left|\left\langle\Phi, T_{K} \Psi\right\rangle\right|<\infty$. Moreover, the CauchySchwarz inequality gives

$$
\begin{aligned}
\left|\left\langle\Phi, T_{K} \Psi\right\rangle\right| & =\left|\int_{X \times X}\langle\Phi(x), K(x, y) \Psi(y)\rangle_{\mathcal{H}} d \mu(y) d \mu(x)\right| \\
\leq & \int_{X \times X}\|\Phi(x)\|_{\mathcal{H}}\|K(x, y)\|_{\mathcal{B}(\mathcal{H})}\|\Psi(y)\|_{\mathcal{H}} d \mu(y) d \mu(x) \\
\leq & \left(\int_{X \times X}\|\Phi(x)\|_{\mathcal{K}}^{2}\|K(x, y)\|_{\mathcal{B}(\mathcal{H})} d \mu(y) d \mu(x)\right)^{1 / 2} \\
& \times\left(\int_{X \times X}\|K(x, y)\|_{\mathcal{B}(\mathcal{H})}\|\Psi(y)\|_{\mathcal{H}}^{2} d \mu(y) d \mu(x)\right)^{1 / 2} \\
\leq & c_{1} c_{2}\|\Phi\|_{L^{2}(X, \mu ; \mathcal{H})}\|\Psi\|_{L^{2}(X, \mu ; \mathcal{H})},
\end{aligned}
$$

and the claim follows immediately.

We are now able to prove that the family of coherent states $\left\{\eta_{x}\right\}_{x \in \mathbb{B}}$ provides a weak resolution of the identity.

Proposition 7.6. Let $\mathbb{B}$ be a normal $\mathbf{j}$-group, $\mathcal{H}$ a separable Hilbert space and $\eta \in \mathcal{D}\left(Q_{N} \times \cdots \times Q_{1}\right) \backslash\{0\}$. Then, for all $\Phi, \Psi \in \mathcal{H}_{\chi} \otimes \mathcal{H}$ the following relation holds:

$$
\langle\Psi, \Phi\rangle_{\mathcal{H}_{\chi} \otimes \mathcal{H}}=C^{\mathbb{B}}(\eta)^{-1} \int_{\mathbb{B}}\left\langle\left\langle\eta_{x}, \Psi\right\rangle_{\mathcal{H}_{\chi}},\left\langle\eta_{x}, \Phi\right\rangle_{\mathcal{H}_{\chi}}\right\rangle_{\mathcal{H}} \mathrm{d}_{\mathbb{B}}(x),
$$

where $\left\langle\eta_{x}, \Psi\right\rangle_{\mathcal{H}_{x}}$ is the vector in $\mathcal{H}$ is defined by:

$$
\left\langle\varphi,\left\langle\eta_{x}, \Psi\right\rangle_{\mathcal{H}_{\chi}}\right\rangle_{\mathcal{H}}:=\left\langle\eta_{x} \otimes \varphi, \Psi\right\rangle_{\mathcal{H}_{\chi} \otimes \mathcal{H}}, \quad \forall \varphi \in \mathcal{H},
$$

and

$$
C^{\mathbb{B}}(\eta):=(2 \pi \theta)^{\operatorname{dim}(\mathbb{B}) / 2}\left\|\Delta_{Q_{N} \times \cdots \times Q_{1}} \eta\right\|_{2}^{2},
$$

where $\Delta_{Q_{N} \times \cdots \times Q_{1}}=\Delta_{Q_{N}} \otimes \cdots \otimes \Delta_{Q_{1}}$, with $\Delta_{Q_{j}}$ the modular function of $Q_{j}$.

\footnotetext{
${ }^{2}$ It can be viewed as a Banach space valued version of Schur's test Lemma.
} 
Proof. We first demonstrate that for $\Phi \in \mathcal{H}_{\chi} \otimes \mathcal{H}$, the map $[x \in \mathbb{B} \mapsto$ $\left.\left\langle\eta_{x}, \Phi\right\rangle_{\mathcal{H}_{\chi}} \in \mathcal{H}\right]$ belongs to $L^{2}(\mathbb{B}, \mathcal{H})$. To see this, let $\left\{\mathbb{B}_{j}\right\}_{j \in \mathbb{N}}$ be an increasing sequence of relatively compact subsets of $\mathbb{B}$, which converges to $\mathbb{B}$. For each $j \in \mathbb{N}$, we define the operator

$$
T_{j}^{\eta}: L^{2}\left(\mathbb{B}_{j}, \mathcal{H}\right) \rightarrow \mathcal{H}_{\chi} \otimes \mathcal{H}, \quad F \mapsto \int_{\mathbb{B}_{j}} \eta_{x} \otimes F(x) \mathrm{d}_{\mathbb{B}}(x) .
$$

Clearly, each $T_{j}^{\eta}$ is bounded:

$$
\begin{aligned}
\left\|T_{j}^{\eta} F\right\|_{\mathcal{H}_{\chi} \otimes \mathcal{H}} \leq & \int_{\mathbb{B}_{j}}\left\|\eta_{x}\right\|_{\mathcal{H}_{\chi}}\|F(x)\|_{\mathcal{H}} \mathrm{d}_{\mathbb{B}}(x) \\
\leq & \|\eta\|_{\mathcal{H}_{\chi}} \operatorname{meas}\left(\mathbb{B}_{j}\right)^{1 / 2}\left(\int_{\mathbb{B}_{j}}\|F(x)\|_{\mathcal{H}}^{2} \mathrm{~d}_{\mathbb{B}}(x)\right)^{1 / 2} \\
& =\|\eta\|_{\mathcal{H}_{\chi}} \operatorname{meas}\left(\mathbb{B}_{j}\right)^{1 / 2}\|F\|_{L^{2}(\mathbb{B}, \mathcal{H})} .
\end{aligned}
$$

To see that the family $\left\{T_{j}^{\eta}\right\}_{j \in \mathbb{N}}$ is in fact uniformly bounded, note that the adjoint of $T_{j}^{\eta}$ reads:

$$
T_{j}^{\eta *}: \mathcal{H}_{\chi} \otimes \mathcal{H} \rightarrow L^{2}\left(\mathbb{B}_{j}, \mathcal{H}\right), \quad \Phi \mapsto\left[x \in \mathbb{B}_{j} \mapsto\left\langle\eta_{x}, \Phi\right\rangle_{\mathcal{H}_{\chi}} \in \mathcal{H}\right] .
$$

Hence for $F \in L^{2}\left(\mathbb{B}_{j}, \mathcal{H}\right)$ we get

$$
\left|T_{j}^{\eta}\right|^{2} F(x)=\int_{\mathbb{B}_{j}}\left\langle\eta_{x}, \eta_{y}\right\rangle F(y) \mathrm{d}_{\mathbb{B}}(y),
$$

that is $\left|T_{j}^{\eta}\right|^{2}=S_{j}^{\eta} \otimes \operatorname{Id}_{\mathcal{H}}$, where $S_{j}^{\eta} \in \mathcal{B}\left(L^{2}\left(\mathbb{B}_{j}\right)\right)$ is a kernel operator with kernel $K_{j}^{\eta}(x, y)=\left\langle\eta_{x}, \eta_{y}\right\rangle$. Applying Lemma 7.5, a simple change of variable gives $\left\|S_{j}^{\eta}\right\| \leq$ $\left\|\left[x \mapsto\left\langle\eta, \eta_{x}\right\rangle\right]\right\|_{1}:=C$ which is finite by Lemma 7.4 and of course, is uniform in $j \in \mathbb{N}$. Finally, since

$$
\int_{\mathbb{B}_{j}}\left\|\left\langle\eta_{x}, \Phi\right\rangle_{\mathcal{H}_{\chi}}\right\|_{\mathcal{H}}^{2} \mathrm{~d}_{\mathbb{B}}(x)=\left\|T_{j}^{\eta *} \Phi\right\|_{L^{2}\left(\mathbb{B}_{j}, \mathcal{H}\right)}^{2} \leq C\|\Phi\|_{\mathcal{H}_{\chi} \otimes \mathcal{H}}^{2},
$$

taking the limit $j \rightarrow \infty$ gives

$$
\int_{\mathbb{B}}\left\|\left\langle\eta_{x}, \Phi\right\rangle_{\mathcal{H}_{\chi}}\right\|_{\mathcal{H}}^{2} \mathrm{~d}_{\mathbb{B}}(x) \leq C\|\Phi\|_{\mathcal{H}_{\chi} \otimes \mathcal{H}}^{2},
$$

as needed. The rest of the proof is computational. Assume first that $\mathbb{B}=\mathbb{S}$ is elementary. In this case, for $\Phi, \Psi \in \mathcal{H}_{\chi} \otimes \mathcal{H}$ and $\eta \in \mathcal{D}(Q)$, we have in chart (2.4) and from (7.3):

$$
\begin{aligned}
& \int_{\mathbb{S}}\left\langle\left\langle\eta_{x}, \Psi\right\rangle_{\mathcal{H}_{x}},\left\langle\eta_{x}, \Phi\right\rangle_{\mathcal{H}_{\chi}}\right\rangle_{\mathcal{H}} \mathrm{d} \mathrm{d}_{\mathbb{S}}(x)= \\
& \int_{\mathbb{R}^{4 d+4}}\left\langle\Psi\left(a_{0}, n_{0}\right), \Phi\left(a_{1}, n_{1}\right)\right\rangle_{\mathcal{H}} \eta\left(a_{0}-a, n_{0}-e^{a-a_{0}} n\right) \bar{\eta}\left(a_{1}-a, n_{1}-e^{a-a_{1}} n\right) \\
& \times \exp \left\{\frac{i}{\theta}\left(\left(e^{-2 a_{0}}-e^{-2 a_{1}}\right) e^{2 a}\left(t+\omega^{0}(n, m)\right)-e^{a} \omega^{0}\left(n_{0} e^{-a_{0}}-n_{1} e^{-a_{1}}, m\right)\right)\right\} \\
& \times \operatorname{d} a \mathrm{~d} n \mathrm{~d} m \mathrm{~d} t \mathrm{~d} a_{0} \mathrm{~d} n_{0} \mathrm{~d} a_{1} \mathrm{~d} n_{1} .
\end{aligned}
$$


Integrating out the $t$-variable yields a factor $2 \pi \theta e^{-2 a+2 a_{0}} \delta\left(a_{0}-a_{1}\right)$, the former expression then becomes

$$
\begin{aligned}
2 \pi \theta \int_{\mathbb{R}^{4 d+2}}\langle\Psi & \left.\left(a_{0}, n_{0}\right), \Phi\left(a_{0}, n_{0}\right)\right\rangle_{\mathcal{H}} \eta\left(a_{0}-a, n_{0}-e^{a-a_{0}} n\right) \bar{\eta}\left(a_{0}-a, n_{1}-e^{a-a_{0}} n\right) \\
& \times e^{-2 a+2 a_{0}} \exp \left\{-\frac{i}{\theta}\left(e^{a-a_{0}} \omega^{0}\left(n_{0}-n_{1}, m\right)\right)\right\} \mathrm{d} a \mathrm{~d} n \mathrm{~d} m \mathrm{~d} a_{0} \mathrm{~d} n_{0} \mathrm{~d} n_{1} .
\end{aligned}
$$

Integrating the $m$-variables, yields a factor $(2 \pi \theta)^{d} e^{-d a+d a_{0}} \delta\left(n_{0}-n_{1}\right)$ and we get, up to a constant:

$$
\begin{gathered}
\int_{\mathbb{R}^{2 d+2}}\left\langle\Psi\left(a_{0}, n_{0}\right), \Phi\left(a_{0}, n_{1}\right)\right\rangle_{\mathcal{H}} \eta\left(a_{0}-a, n_{0}-e^{a-a_{0}} n\right) \bar{\eta}\left(a_{0}-a, n_{0}-e^{a-a_{0}} n\right) \\
\times e^{-(d+2)\left(a-a_{0}\right)} \mathrm{d} a \mathrm{~d} n \mathrm{~d} a_{0} \mathrm{~d} n_{0},
\end{gathered}
$$

which after an affine change of variable and restoring the constants, gives

$$
(2 \pi \theta)^{d+1}\langle\Psi, \Phi\rangle_{\mathcal{H}_{\chi} \otimes \mathcal{H}} \int_{\mathbb{R}^{d+1}}|\eta(a, n)|^{2} e^{(2 d+2) a} \mathrm{~d} a \mathrm{~d} n
$$

which is all we needed since $\Delta_{Q}(a, n)=e^{(d+1) a}$.

The case of a generic normal $\mathbf{j}$-group $\mathbb{B}$ is treated with the same argument lines as in Proposition 7.3 , set $\mathbb{B}^{\prime} \ltimes \mathbb{S}_{1}$, with $\mathbb{S}_{1}$ elementary normal and assume that the relation (7.8) holds for $\mathbb{B}^{\prime}$. With the notations of (7.5), we have for all $\varphi=\varphi^{\prime} \otimes \varphi^{1}, \psi=$ $\psi^{\prime} \otimes \psi^{1} \in \mathcal{H}_{\chi}$ and omitting the constant $\left.C^{\mathbb{B}}(\eta)^{-1}=C^{\mathbb{B}^{\prime}}\left(\eta^{\prime}\right)^{-1} C^{\mathbb{S}_{1}}\left(\eta^{1}\right)^{-1}\right)$ :

$$
\begin{aligned}
& \int_{\mathbb{B}}\left\langle\varphi, \eta_{x}\right\rangle\left\langle\eta_{x}, \psi\right\rangle \mathrm{d}_{\mathbb{B}}(x) \\
& =\int_{\mathbb{B}^{\prime} \times \mathbb{S}_{1}}\left\langle\varphi^{\prime}, \eta_{g^{\prime}}^{\prime}\right\rangle\left\langle\varphi^{1}, \mathcal{R}\left(g^{\prime}\right) \eta_{g_{1}}^{1}\right\rangle\left\langle\mathcal{R}\left(g^{\prime}\right) \eta_{g_{1}}^{1}, \psi^{1}\right\rangle\left\langle\eta_{g^{\prime}}^{\prime}, \psi^{\prime}\right\rangle \mathrm{d}_{\mathbb{B}^{\prime}}\left(g^{\prime}\right) \mathrm{d}_{\mathbb{S}_{1}}\left(g_{1}\right) \\
& =\int_{\mathbb{B}^{\prime}}\left\langle\varphi^{\prime}, \eta_{g^{\prime}}^{\prime}\right\rangle\left(\int_{\mathbb{S}_{1}}\left\langle\mathcal{R}\left(g^{\prime-1}\right) \varphi^{1}, \eta_{g_{1}}^{1}\right\rangle\left\langle\eta_{g_{1}}^{1}, \mathcal{R}\left(g^{\prime-1}\right) \psi^{1}\right\rangle \mathrm{d}_{\mathbb{S}_{1}}\left(g_{1}\right)\right)\left\langle\eta_{g^{\prime}}^{\prime}, \psi^{\prime}\right\rangle \mathrm{d}_{\mathbb{B}^{\prime}}\left(g^{\prime}\right) \\
& =\int_{\mathbb{B}^{\prime}}\left\langle\varphi^{\prime}, \eta_{g^{\prime}}^{\prime}\right\rangle\left\langle\mathcal{R}\left(g^{\prime}{ }^{\prime-1}\right) \varphi^{1}, \mathcal{R}\left(g^{\prime-1}\right) \psi^{1}\right\rangle\left\langle\eta_{g^{\prime}}^{\prime}, \psi^{\prime}\right\rangle \mathrm{d}_{\mathbb{B}^{\prime}}\left(g^{\prime}\right) \\
& =\left\langle\varphi^{1}, \psi^{1}\right\rangle \int_{\mathbb{B}^{\prime}}\left\langle\varphi^{\prime}, \eta_{g^{\prime}}^{\prime}\right\rangle\left\langle\eta_{g^{\prime}}^{\prime}, \psi^{\prime}\right\rangle \mathrm{d} g^{\prime}=\left\langle\varphi^{1}, \psi^{1}\right\rangle\left\langle\varphi^{\prime}, \psi^{\prime}\right\rangle=\langle\varphi, \psi\rangle .
\end{aligned}
$$

REMARK 7.7. In the following, we will absorb the constant $C(\eta)^{1 / 2}$ of Proposition [7.6] in a redefinition of the mother wavelet $\eta \in \mathcal{D}\left(Q_{N} \times \cdots \times Q_{1}\right)$.

REMARK 7.8. Other types of weak resolution of the identity can be constructed in this setting. For instance, setting $\tilde{\eta}_{x}:=\Omega_{\mathbf{m}_{0}}^{\mathbb{B}}(x) \eta$, where $\eta$ is arbitrary in $\mathcal{H}_{\chi}$, we have from the unitarity of the quantization map $\Omega_{\mathbf{m}_{0}}^{\mathbb{B}}$ :

$$
\langle\psi, \phi\rangle=\|\eta\|^{-2} \int_{\mathbb{B}}\left\langle\psi, \tilde{\eta}_{x}\right\rangle\left\langle\tilde{\eta}_{x}, \phi\right\rangle \mathrm{d}_{\mathbb{B}}(x),
$$

for all $\phi, \psi \in \mathcal{H}_{\chi}$. Similarly, let $W_{x, y}^{\eta}$ be the Wigner function on $\mathbb{B}$, associated to a pair of wavelets $\eta_{x}, \eta_{y}$ :

$$
W_{x, y}^{\eta}(z):=\sigma_{\mathbf{m}_{0}}\left[\left|\eta_{x}\right\rangle\left\langle\eta_{y}\right|\right](z)=\left\langle\eta_{y}\left|\Omega_{\mathbf{m}_{0}}(z)\right| \eta_{x}\right\rangle, \quad x, y, z \in \mathbb{B} .
$$


Then, these Wigner functions may be used to construct a weak resolution of the identity on $L^{2}(\mathbb{B})$ : For all $f_{1}, f_{2} \in L^{2}(\mathbb{B})$, we have

$$
\left\langle f_{1}, f_{2}\right\rangle=\|\eta\|^{-4} \int_{\mathbb{B} \times \mathbb{B}}\left\langle f_{1}, W_{x, y}^{\eta}\right\rangle\left\langle W_{x, y}^{\eta}, f_{2}\right\rangle \mathrm{d}_{\mathbb{B}}(x) \mathrm{d}_{\mathbb{B}}(y) .
$$

Proposition 7.9. Let $\mathbb{B}$ be a normal $\mathbf{j}$-group, $\mathcal{H}$ be a separable Hilbert space and $A$ be a densely defined operator on $\mathcal{H}_{\chi} \otimes \mathcal{H}$, whose domain contains the (algebraic) tensor product $\mathcal{D}\left(Q_{N} \times \cdots \times Q_{1}\right) \otimes \mathcal{H}$. For $x, y \in \mathbb{B}$ and $\eta \in \mathcal{D}\left(Q_{N} \times \cdots \times Q_{1}\right)$ define the element $\left\langle\eta_{x}, A \eta_{y}\right\rangle_{\mathcal{H}_{x}}$ of $\mathcal{B}(\mathcal{H})$ by means of the quadratic form

$$
\mathcal{H} \times \mathcal{H} \rightarrow \mathbb{C}, \quad(\phi, \psi) \mapsto\left\langle\eta_{x} \otimes \phi, A \eta_{y} \otimes \psi\right\rangle_{\mathcal{H}_{x} \otimes \mathcal{H}} .
$$

Assuming further that

$$
\sup _{y \in \mathbb{B}} \int_{\mathbb{B}}\left\|\left\langle\eta_{x}, A \eta_{y}\right\rangle_{\mathcal{H}_{\chi}}\right\|_{\mathcal{B}(\mathcal{H})} \mathrm{d}_{\mathbb{B}}(x)<\infty, \quad \sup _{x \in \mathbb{B}} \int_{\mathbb{B}}\left\|\left\langle\eta_{x}, A \eta_{y}\right\rangle_{\mathcal{H}_{\chi}}\right\|_{\mathcal{B}(\mathcal{H})} \mathrm{d}_{\mathbb{B}}(y)<\infty,
$$

then $A$ extends to a bounded operator on $\mathcal{H}_{\chi} \otimes \mathcal{H}$.

Proof. Since $\eta_{x}$ is smooth and compactly supported, our assumption about the domain of $A$ ensures that $\left\langle\eta_{x}, A \eta_{y}\right\rangle_{\mathcal{H}_{x}}$ is well defined as an element of $\mathcal{B}(\mathcal{H})$. Thus, Lemma 7.5 applied to $(X, \mu)=\left(\mathbb{B}, \mathrm{d}_{\mathbb{B}}\right)$, yields that the operator $\tilde{A}$ on $L^{2}(\mathbb{B}, \mathcal{H})$ given by

$$
\tilde{A} F(x):=\int_{\mathbb{B}}\left\langle\eta_{x}, A \eta_{y}\right\rangle_{\mathcal{H}_{\chi}} F(y) d \mu(y),
$$

and is bounded, with

$$
\begin{aligned}
&\|\tilde{A}\| \leq\left(\sup _{y \in \mathbb{B}} \int_{\mathbb{B}}\left\|\left\langle\eta_{x}, A \eta_{y}\right\rangle_{\mathcal{H}_{\chi}}\right\|_{\mathcal{B}(\mathcal{H})} \mathrm{d}_{\mathbb{B}}(x)\right)^{1 / 2} \\
& \times\left(\sup _{x \in \mathbb{B}} \int_{\mathbb{B}}\left\|\left\langle\eta_{x}, A \eta_{y}\right\rangle_{\mathcal{H}_{\chi}}\right\|_{\mathcal{B}(\mathcal{H})} \mathrm{d}_{\mathbb{B}}(y)\right)^{1 / 2}<\infty .
\end{aligned}
$$

For $\Phi \in \mathcal{H}_{\chi} \otimes \mathcal{H}$ define the $\mathcal{H}$-valued function on $\mathbb{B}: \tilde{\Phi}:=\left[x \in \mathbb{B} \mapsto\left\langle\eta_{x}, \Phi\right\rangle_{\mathcal{H}_{\chi}} \in\right.$ $\mathcal{H}$ ]. By Proposition 7.6 we know that $\tilde{\Phi}$ belongs to $L^{2}(\mathbb{B}, \mathcal{H})$ with $\|\Phi\|_{\mathcal{H}_{\chi} \otimes \mathcal{H}}=$ $\|\tilde{\Phi}\|_{L^{2}(\mathbb{B}, \mathcal{H})}$. Take now $\Phi, \Psi \in \operatorname{dom} A$. In this case, we can use twice the resolution of the identity to get

$$
\begin{aligned}
\langle\Phi, A \Psi\rangle_{\mathcal{H}_{\chi} \otimes \mathcal{H}} & =\int_{\mathbb{B} \times \mathbb{B}}\left\langle\left\langle\eta_{x}, \Phi\right\rangle_{\mathcal{H}_{\chi}},\left\langle\eta_{x}, A \eta_{y}\right\rangle_{\mathcal{H}_{\chi}}\left\langle\eta_{y}, \Psi\right\rangle_{\mathcal{H}_{\chi}}\right\rangle_{\mathcal{H}} \mathrm{d}_{\mathbb{B}}(x) \mathrm{d}_{\mathbb{B}}(y) \\
& =\langle\tilde{\Phi}, \tilde{A} \tilde{\Psi}\rangle_{L^{2}(\mathbb{B}, \mathcal{H})} .
\end{aligned}
$$

Therefore, we conclude that

$$
\begin{aligned}
\left|\langle\Phi, A \Psi\rangle_{\mathcal{H}_{\chi} \otimes \mathcal{H}}\right| & =\left|\langle\tilde{\Phi}, \tilde{A} \tilde{\Psi}\rangle_{L^{2}(\mathbb{B}, \mathcal{H})}\right| \\
& \leq\|\tilde{\Phi}\|_{L^{2}(\mathbb{B}, \mathcal{H})}\|\tilde{\Psi}\|_{L^{2}(\mathbb{B}, \mathcal{H})}\|\tilde{A}\|=\|\Phi\|_{\mathcal{H}_{\chi} \otimes \mathcal{H}}\|\Psi\|_{\mathcal{H}_{\chi} \otimes \mathcal{H}}\|\tilde{A}\|<\infty
\end{aligned}
$$

and the result follows immediately.

\subsection{A tempered pair from the one-point phase}

Let $\mathbb{S}$ be an elementary normal $\mathbf{j}$-group. Consider the one-point phase $\mathbf{E}_{\theta}$, defined in (5.11), and given by

$$
\mathbf{E}_{\theta}(q b)=\bar{\chi}_{\theta}\left(\mathbf{C}_{q}\left(b^{-1} \tilde{\sigma} b\right)\right)=: e^{\frac{i}{\theta} \mathbf{S}(q b)} .
$$


Recall that from Lemma 6.14, we have in the coordinates (2.4) and up to a global constant factor:

$$
\mathbf{S}(a, n \oplus m, t)=t \sinh 2 a+\omega^{0}(n, m) \cosh ^{2} a .
$$

The aim of this section it to prove that the pair $(\mathbb{S}, \mathbf{S})$, is tempered, admissible and tame. For this, we consider the following decomposition of the Lie algebra $\mathfrak{s}$ (i.e. the one we used in Equation (2.10) ):

$$
\mathfrak{s}=\bigoplus_{k=0}^{3} V_{k} \quad \text { where } \quad V_{0}:=\mathfrak{a}, \quad V_{1}:=\mathfrak{l}^{\star}, \quad V_{2}:=\mathfrak{l} \quad \text { and } \quad V_{3}:=\mathbb{R} E .
$$

As usual, we us fix $\left\{f_{j}\right\}_{j=1}^{d}$, a basis of $\mathfrak{l}^{\star}$ to which we associate $\left\{e_{j}\right\}_{j=1}^{d}$ the symplectic-dual basis of $\mathfrak{l}$, defined by $\omega^{0}\left(f_{i}, e_{j}\right)=\delta_{i, j}$. Associated to the decomposition $v=n \oplus m \in \mathfrak{l}^{\star} \oplus \mathfrak{l}=V$, we get coordinates

$$
n_{j}:=\omega^{0}\left(n, e_{j}\right), \quad m_{j}:=\omega^{0}\left(f_{j}, m\right), \quad j=1, \ldots, d .
$$

From the expressions (2.8) of the left invariant vector fields of $\mathbb{S}$, in the chart (2.4), we get the following coordinates system on $\mathbb{S}$ :

$$
\begin{aligned}
x_{0}:=\widetilde{H} \mathbf{S}=2 e^{-2 a} t-\left(1+e^{-2 a}\right) \omega^{0}(n, m), & x_{1}^{j}:=\widetilde{f}_{j} \mathbf{S}=\left(1+e^{-2 a}\right) m_{j}, \\
x_{2}^{j}:=\widetilde{e}_{j} \mathbf{S}=\left(1+e^{2 a}\right) n_{j}, & x_{3}:=\widetilde{E} \mathbf{S}=\sinh 2 a .
\end{aligned}
$$

We then deduce:

Lemma 7.10. The pair $(\mathbb{S}, \mathbf{S})$ is tempered in the sense of Definition 1.22. Moreover, the Jacobian of the map

$$
\phi: \mathbb{S} \rightarrow \mathfrak{s}^{\star}, g \mapsto[\mathfrak{s} \rightarrow \mathbb{R}, X \in \mathfrak{s} \mapsto(\widetilde{X} \mathbf{S})(g)],
$$

is proportional to $\mathbf{m}_{0}^{2} \times \Delta_{\mathbb{S}}^{-1 /(d+1)}$.

The following Lemma is actually all that we need to prove admissibility (in the sense of Definition 1.24) of the tempered pair $(\mathbb{S}, \mathbf{S})$ :

Lemma 7.11. For every $k \in\{0,1,2,3\}$, there exists a tempered function $\mathbf{m}_{k}>0$ with $\partial_{x_{j}} \mathbf{m}_{k}=0$ for every $j \leq k$ and such that for every $X \in \mathcal{U}\left(V^{(k)}\right)$, there exists $C_{X}>0$ with

$$
\left|\widetilde{X} x_{k}\right| \leq C_{X} \mathbf{m}_{k}\left(1+\left|x_{k}\right|\right)
$$

Proof. From the computations, for $k \in \mathbb{N}^{*}$ and $i, j=1, \ldots, d$ :

$$
\begin{aligned}
\widetilde{H}^{k} x_{0} & =(-1)^{k}\left(2^{2 k+1} e^{-2 a} t-2^{k}\left(1+2^{k} e^{-2 a}\right) \omega^{0}(n, m)\right), \\
\widetilde{H}^{k} x_{1}^{j} & =(-1)^{k}\left(1+3^{k} e^{-2 a}\right) m_{j}, \quad \widetilde{f}_{i} x_{1}^{j}=0, \\
\widetilde{H}^{k} x_{2}^{j} & =\left((-1)^{k}+e^{2 a}\right) n_{j}, \quad \widetilde{f}_{i} x_{2}^{j}=\left(1+e^{2 a}\right) \delta_{i}^{j}, \quad \widetilde{e}_{i} x_{2}^{j}=0, \\
\widetilde{H}^{k} x_{3} & =2^{k+1}\left\{\begin{array}{ll}
\cosh 2 a, & k \text { even } \\
\sinh 2 a, & k \text { odd }
\end{array}, \quad \widetilde{f}_{i} x_{3}=0, \quad \widetilde{e}_{i} x_{3}=0, \quad \widetilde{E} x_{3}=0,\right.
\end{aligned}
$$


and elementary estimates, we obtain:

$$
\begin{array}{ll}
\left|\tilde{X} x_{0}\right| \leq C_{X}^{0}\left(1+\left|x_{0}\right|\right)\left(1+\left|x_{1}\right|\left|x_{2}\right|\right), & \forall X \in \mathcal{U}\left(V_{0}\right), \\
\left|\widetilde{X} x_{1}^{j}\right| \leq C_{X}^{1}\left(1+\left|x_{1}^{j}\right|\right), & \forall X \in \mathcal{U}\left(V^{(1)}\right), \\
\left|\tilde{X} x_{2}^{j}\right| \leq C_{X}^{2}\left(1+\left|x_{2}^{j}\right|\right)\left(1+\left|x_{3}\right|\right), & \forall X \in \mathcal{U}\left(V^{(2)}\right), \\
\left|\widetilde{X} x_{3}\right| & \leq C_{X}^{3}\left(1+\left|x_{3}\right|\right), \quad \forall X \in \mathcal{U}(\mathfrak{s}),
\end{array}
$$

and the claim follows with $\mathbf{m}_{0}(x)=\left(1+\left|x_{1}\right|\left|x_{2}\right|\right), \mathbf{m}_{1}(x)=1, \mathbf{m}_{2}(x)=\left(1+\left|x_{3}\right|\right)$, $\mathbf{m}_{3}(x)=1$.

Repeating the arguments of the proof of Proposition 2.26, we deduce admissibility for the tempered pair $(\mathbb{S}, \mathbf{S})$.

Lemma 7.12. Define

$$
\begin{aligned}
& X_{0}:=1-H^{2} \in \mathcal{U}\left(V_{0}\right), \quad X_{1}:=1-\sum_{j=1}^{d} f_{j}^{2} \in \mathcal{U}\left(V_{1}\right), \\
& X_{2}:=1-\sum_{j=1}^{d} e_{j}^{2} \in \mathcal{U}\left(V_{2}\right), \quad X_{3}:=1-E^{2} \in \mathcal{U}\left(V_{3}\right) .
\end{aligned}
$$

Then the corresponding multipliers $\alpha_{k}:=\mathbf{E}^{-1} \widetilde{X}_{k} \mathbf{E}$ satisfy conditions (i) and (ii) of Definition 1.24, with $\rho_{k}=2$ and the $\mu_{k}$ 's are given by the $\mathbf{m}_{k}$ 's of Lemma 7.11.

Lastly, we observe that tameness (see Definition 1.34) follows from Lemma 2.27 and arguments very similar to those of Corollary 2.28. We then summarize all this by stating the main result of this section:

TheOrem 7.13. Let $\mathbb{S}$ be an elementary normal $\mathbf{j}$-group and let $\mathbf{S} \in C^{\infty}(\mathbb{S})$ be as given in (7.9). Then the pair $(\mathbb{S}, \mathbf{S})$ is tempered, admissible and tame.

Remark 7.14. For $\mathbb{B}$ a generic normal $\mathbf{j}$-group $\mathbb{B}$, we could also define a onepoint tempered pair, by setting

$$
\mathbf{E}_{\theta}^{\mathbb{B}}:=\exp \left\{\frac{2 i}{\theta} \mathbf{S}^{\mathbb{B}}\right\}: \mathbb{B} \rightarrow \mathbb{U}(1), \quad \mathbf{S}^{\mathbb{B}}: \mathbb{B} \rightarrow \mathbb{R}, \quad g \mapsto \sum_{j=1}^{N} \mathbf{S}^{\mathbb{S}_{j}}\left(g_{j}\right),
$$

where $\mathbf{S}^{\mathbb{S}_{j}}$ is the one-point phase (7.9) of each elementary factor of $\mathbb{B}$ in the parametrization $g=g_{1} \ldots g_{N} \in \mathbb{B}$, relative to a Pyatetskii-Shapiro decomposition. Then temperedness and admissibility will follow from arguments very similar than those of Theorem 2.35.

REMARK 7.15. The one-point Schwartz space $\mathcal{S}^{S_{\text {can }}}(\mathbb{S})$ associated with the twopoint pair $\left(\mathbb{S} \times \mathbb{S}, S_{\text {can }}\right)$ as given in Definition 1.48 coincides with the one-point Schwartz space $\mathcal{S}^{\mathbf{S}}(\mathbb{S})$ associated with the one-point par $(\mathbb{S}, \mathbf{S})$.

\subsection{Extension of the oscillatory integral}

Associated to a tempered, admissible and tame pair $(G, S)$, we have constructed in section 1.3 a continuous linear map for any Fréchet space $\mathcal{E}$ and any element $\mathbf{m} \in \mathcal{B}^{\mu}(G)$ (with $\mu$ a tempered weight on $G$ ):

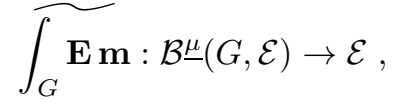


which extends the ordinary integral on $\mathcal{D}(G, \mathcal{E})$ and that we called the oscillatory integral.

The aim of the present section is to explain how for the tempered pair $(\mathbb{S}, \mathbf{S})$ of Theorem 7.13, one can enlarge the domain of definition of the oscillatory integral. For this let $\mathcal{E}$ be a Fréchet space, $\underline{\mu}=\left\{\mu_{j}\right\}_{j \in \mathbb{N}}$ a family of tempered weights on $\mathbb{S}$ and $\nu$ be a fixed tempered and $\mathbb{Y}$-right-invariant weigh $t 3$ on $\mathbb{S}$. Let us then consider the following subspace of $C^{\infty}(\mathbb{S}, \mathcal{E})$ :

$$
\begin{array}{r}
\mathcal{B}^{\mu}, \nu(\mathbb{S}, \mathcal{E}):=\left\{F \in C^{\infty}(\mathbb{S}, \mathcal{E}): \forall(j, X, Y) \in \mathbb{N} \times \mathcal{U}(\mathfrak{q}) \times \mathcal{U}(\mathfrak{Y}), \exists C:\right. \\
\left.\|\widetilde{X} \widetilde{Y} F(q b)\|_{j} \leq C \nu(q)^{\operatorname{deg}(X)} \mu_{j}(q b)\right\} .
\end{array}
$$

This space may be understood as a variant of the symbol space $\mathcal{B}-\underline{\mu}(\mathbb{S}, \mathcal{E})$, where a specific dependence of the family of weights $\mu$ in the degree of the derivative is allowed. We endow the latter space with the following set of semi-norms:

$$
\|F\|_{j, k_{1}, k_{2}, \underline{\mu}, \nu}:=\sup _{X \in \mathcal{U}_{k_{1}}(\mathfrak{q})} \sup _{Y \in \mathcal{U}_{k_{2}}(\mathfrak{Y})} \sup _{q b \in \mathbb{S}}\left\{\frac{\|\widetilde{X} \tilde{Y} F(q b)\|_{j}}{\mu_{j}(q b) \nu(q)^{k_{1}}|X|_{k_{1}}|Y|_{k_{2}}}\right\},
$$

where $j, k_{1}, k_{2} \in \mathbb{N}$, and $\bigcup_{k \in \mathbb{N}} \mathcal{U}_{k}(\mathfrak{q}), \bigcup_{k \in \mathbb{N}} \mathcal{U}_{k}(\mathfrak{Y})$ are the filtrations of $\mathcal{U}(\mathfrak{q})$ and $\mathcal{U}(\mathfrak{Y})$ associated to the choice of PBW basis as explained in (0.4). As expected, the space $\mathcal{B}^{\mu}, \nu(\mathbb{S}, \mathcal{E})$ is Fréchet for the topology induced by the semi-norms (17.12) and most the properties of Lemma 1.12 remain true.

Lemma 7.16. Let $(\mathbb{S}, \mathcal{E})$ be as above, let $\underline{\mu}, \underline{\rho}$ and $\underline{\hat{\mu}}$ be three families of weights on $\mathbb{S}$ and let $\nu, \lambda$ and $\hat{\nu}$ be three right- $\mathbb{Y}$-invariant weights on $\mathbb{S}$.

(i) The space $\mathcal{B}^{\mu}, \nu(\mathbb{S}, \mathcal{E})$ is Fréchet.

(ii) The bilinear map:

$\mathcal{B}^{\underline{\mu}, \nu}(\mathbb{S}) \times \mathcal{B}^{\rho}, \lambda(\mathbb{S}, \mathcal{E}) \rightarrow \mathcal{B}^{\mu} \cdot \underline{\rho}, \nu \cdot \lambda(\mathbb{S}, \mathcal{E}), \quad(u, F) \mapsto[g \in \mathbb{S} \mapsto u(g) F(g) \in \mathcal{E}]$, is continuous.

(iii) If there exists $C>0$ such that $\underline{\mu} \leq C \underline{\hat{\mu}}$ and $\nu \leq C \hat{\nu}$, then $\mathcal{B} \underline{\mu}, \nu(\mathbb{S}, \mathcal{E}) \subset$ $\mathcal{B} \underline{\hat{\mu}}, \hat{\nu}(\mathbb{S}, \mathcal{E})$ continuously.

(iv) Assume that $\mu \prec \mu$ and $\nu \prec \hat{\nu}$. Then, the closure of $\mathcal{D}(\mathbb{S}, \mathcal{E})$ in $\mathcal{B} \underline{\hat{\mu}}, \hat{\nu}(\mathbb{S}, \mathcal{E})$ contains $\mathcal{B}^{\mu}, \nu(\mathbb{S}, \mathcal{E})$. In particular, $\mathcal{D}(\mathbb{S}, \mathcal{E})$ is a dense subset of $\mathcal{B}^{\mu}, \nu(\mathbb{S}, \mathcal{E})$ for the induced topology of $\mathcal{B} \underline{\hat{\mu}} \hat{\nu}(\mathbb{S}, \mathcal{E})$.

Proof. The first assertion follows from the fact that a countable projective limit of Fréchet spaces is Fréchet and that $\mathcal{B} \underline{\mu}, \nu(\mathbb{S}, \mathcal{E})$ can be realized as the countable projective limit of the family of Banach spaces underlying the norms $\sum_{i=0}^{j} \sum_{l_{1}=0}^{k_{1}} \sum_{l_{2}=0}^{k_{2}}\|\cdot\|_{i, l_{1}, l_{2}, \underline{\mu}, \nu}$. The proof of all the other statements are identical to their counter-parts in Lemma 1.12 .

We are now able to prove our extension result for the oscillatory integral associated to the admissible, tempered and tame pair $(\mathbb{S}, \mathbf{S})$ :

THEOREM 7.17. Let $\underline{\mu}$ be family of tempered weights on $\mathbb{S}, \nu$ a $\mathbb{Y}$-right-invariant tempered weight on $\mathbb{S}$ an $\bar{d} \mathbf{m}$ an element of $\mathcal{B}^{\lambda}(\mathbb{S})$ for another tempered weight $\lambda$ on $\mathbb{S}$. Let also $\mathbf{D}_{\vec{r}}, \vec{r} \in \mathbb{N}^{4}$, be the differential operator constructed in (1.22). Then for

\footnotetext{
${ }^{3}$ We may view $\nu$ as a function on $Q$.
} 
all $j \in \mathbb{N}$, there exist $\vec{r}_{j} \in \mathbb{N}^{4}, C_{j}>0$ and $k_{j}, l_{j} \in \mathbb{N}$, such that for every element $F \in \mathcal{B}^{\mu}, \nu(\mathbb{S}, \mathcal{E})$, we have

$$
\int_{\mathbb{S}}\left\|\mathbf{D}_{\vec{r}} \mathbf{m}(g) F(g)\right\|_{j} \mathrm{~d}_{\mathbb{S}}(g) \leq C_{j}\|F\|_{j, k_{j}, l_{j}, \mu, \nu} .
$$

Consequently, the oscillatory integral constructed in Definition 1.31 for the tame and admissible tempered pair $(\mathbb{S}, \mathbf{S})$, originally defined in $\mathcal{B} \underline{\mu}(\mathbb{S}, \mathcal{E})$, extends as a continuous map:

$$
\widetilde{\int_{\mathbb{S}} \mathbf{m} \mathbf{E}: \mathcal{B} \underline{\mu}, \nu}(\mathbb{S}, \mathcal{E}) \rightarrow \mathcal{E}
$$

Proof. The proof is very similar to those of Proposition 1.28 so we focus on the differences due to the particular behavior at infinity of an element of $\mathcal{B} \underline{\mu}, \nu(\mathbb{S}, \mathcal{E})$.

By Lemma 7.10, the Radon-Nikodym derivative of the left Haar measure on $\mathbb{S}$ with respect to the Lebesgue measure on $\mathfrak{s}^{\star}$, is bounded by a polynomial of order $2 d+4$ in the coordinate $x_{3}$. For each $j \in \mathbb{N}$, the weight $\mu_{j}$ is also bounded by a polynomial in $x_{0}, x_{1}, x_{2}, x_{3}$. Now, observe that by construction of the operator $\mathbf{D}_{\vec{r}}$ in (1.22), we have for any $\vec{r}=\left(r_{0}, r_{1}, r_{2}, r_{3}\right) \in \mathbb{N}^{4}$, with $K_{1}=2 r_{0}+2 r_{1}$, $K_{2}=2 r_{2}+2 r_{3}$ and with the notations given in (1.24):

$$
\begin{aligned}
\left|\mathbf{D}_{\vec{r}} F\right| & \leq\left|\Psi_{0}\right|\left|\Psi_{1,0}\right|\left|\Psi_{2,1,0}\right|\left|\Psi_{3,2,1,0}\right|\left|\widetilde{X}_{3,2,1,0}^{\prime} F\right| \\
& \leq C\left|\Psi_{0}\right|\left|\Psi_{1,0}\right|\left|\Psi_{2,1,0}\right|\left|\Psi_{3,2,1,0}\right| \mu_{j} \nu^{2 r_{0}+2 r_{1}}\|F\|_{j, K_{1}, K_{2}, \underline{\mu}, \nu} .
\end{aligned}
$$

This will gives the estimate we need, if we prove that the function in front of $\|F\|_{j, K_{1}, K_{2}, \underline{\mu}, \nu}$ in (7.13) is integrable for a suitable choice of $\vec{r} \in \mathbb{N}^{4}$. We prove a stronger result, namely that given $\vec{R} \in \mathbb{N}^{4}$, there exists $\vec{r} \in \mathbb{N}^{4}$ such that

$$
\begin{aligned}
& \left|\Psi_{0}\right|\left|\Psi_{1,0}\right|\left|\Psi_{2,1,0}\right|\left|\Psi_{3,2,1,0}\right| \nu^{2 r_{0}+2 r_{1}} \\
& \leq \frac{C}{\left(1+\left|x_{0}\right|\right)^{R_{0}}\left(1+\left|x_{1}\right|\right)^{R_{1}}\left(1+\left|x_{2}\right|\right)^{R_{2}}\left(1+\left|x_{3}\right|\right)^{R_{3}}} .
\end{aligned}
$$

From Corollary 1.27 and Lemma 7.12, we obtain the following estimation:

$$
\begin{aligned}
& \left|\Psi_{0}\right|\left|\Psi_{1,0}\right|\left|\Psi_{2,1,0}\right|\left|\Psi_{3,2,1,0}\right| \\
& \leq C \frac{\left(1+\left|x_{1}\right|\left|x_{2}\right|\right)^{2 r_{0}^{2}}}{\left(1+\left|x_{0}\right|\right)^{2 r_{0}}} \frac{1}{\left(1+\left|x_{1}\right|\right)^{2 r_{1}}} \frac{\left(1+\left|x_{3}\right|\right)^{2 r_{2}\left(r_{0}+r_{1}+r_{2}\right)}}{\left(1+\left|x_{2}\right|\right)^{2 r_{2}}} \frac{1}{\left(1+\left|x_{3}\right|\right)^{2 r_{3}}} .
\end{aligned}
$$

Lastly (this is the main difference with the proof of Proposition 1.28), note that $\nu$, the tempered function on $Q$, can be bounded by $\left|x_{2}\right|^{p_{2}}\left|x_{3}\right|^{p_{3}}$ for some integers $p_{2}, p_{3}$. Hence $\left|\Psi_{0}\right|\left|\Psi_{1,0}\right|\left|\Psi_{2,1,0}\right|\left|\Psi_{3,2,1,0}\right| \nu^{2 r_{0}+2 r_{1}}$ is smaller than

$$
\begin{aligned}
C\left(1+\left|x_{0}\right|\right)^{-2 r_{0}}\left(1+\left|x_{1}\right|\right)^{-2 r_{1}+2 r_{0}^{2}}\left(1+\left|x_{2}\right|\right)^{-2 r_{2}+2 r_{0}^{2}+2 p_{2}\left(r_{0}+r_{1}\right)} \\
\times\left(1+\left|x_{3}\right|\right)^{-2 r_{3}+2 r_{2}\left(r_{0}+r_{1}+r_{2}\right)+2 p_{3}\left(r_{0}+r_{1}\right),}
\end{aligned}
$$

and the claim follows.

\subsection{A Calderón-Vaillancourt type estimate}

For $j=1, \ldots, N$, fix $\mathbf{m}_{j}$ a $\mathbb{Y}_{j}$-right-invariant tempered weight on $\mathbb{S}_{j}$ (that we identify in a natural manner as a function on $Q_{j}$ ), in the sense of Definition 1.17 for the tempered pair $\left(\mathbb{S}_{j}, \mathbf{S}^{\mathbb{S}_{j}}\right)$ underlying Theorem 7.13 , Let also $A$ be a $C^{*}$ subalgebra of $\mathcal{B}(\mathcal{H})$, with $\mathcal{H}$ a separable Hilbert space. Our aim here is to prove that for $F \in \mathcal{B}(\mathbb{B}, A)$, the operator $\Omega_{\theta, \mathbf{m}}(F)$, defined via a suitable quadratic form 
on $\mathcal{H}_{\chi} \otimes \mathcal{H}$, is bounded 4 . We start by proceeding formally, in order to explain our global strategy. Also, to simplify the notations, we assume first that $\mathbb{B}=\mathbb{S}$ is elementary. So let $\Phi, \Psi \in \mathcal{H}_{\chi} \otimes \mathcal{H}$. Using twice the resolution of the identity of Proposition 7.6, we write

$$
\begin{aligned}
& \left\langle\Phi, \Omega_{\theta, \mathbf{m}}(F) \Psi\right\rangle_{\mathcal{H}_{\chi} \otimes \mathcal{H}}= \\
& \quad \int_{\mathbb{S} \times \mathbb{S}}\left\langle\left\langle\eta_{x}, \Phi\right\rangle_{\mathcal{H}_{\chi}},\left\langle\eta_{x}, \Omega_{\theta, \mathbf{m}}(F) \eta_{y}\right\rangle_{\mathcal{H}_{\chi}}\left\langle\eta_{y}, \Psi\right\rangle_{\mathcal{H}_{\chi}}\right\rangle_{\mathcal{H}} \mathrm{d}_{\mathbb{S}}(x) \mathrm{d} \mathrm{d}_{\mathbb{S}}(y) .
\end{aligned}
$$

Next, we use the $\mathbb{S}$-covariance of the pseudo-differential calculus to get

$$
\left\langle\eta_{x}, \Omega_{\theta, \mathbf{m}}(F) \eta_{y}\right\rangle_{\mathcal{H}_{\chi}}=\left\langle\eta_{y^{-1} x}, \Omega_{\theta, \mathbf{m}}\left(L_{y^{-1}}^{\star} F\right) \eta\right\rangle_{\mathcal{H}_{\chi}} .
$$

Then, we exchange the integrals over $\mathbb{S}$ and $Q$ and expand the scalar product of $\mathcal{H}_{\chi}$ to obtain:

$$
\left\langle\eta_{x}, \Omega_{\theta, \mathbf{m}}(F) \eta\right\rangle_{\mathcal{H}_{\chi}}=\int_{\mathbb{S} \times Q} F\left(q_{0} q b\right) \overline{\eta_{x}}\left(q_{0}\right) \mathbf{m}\left(q^{-1}\right) \mathbf{E}(q b) \eta\left(q_{0} \underline{s}^{e} q\right) \mathrm{d}_{Q}\left(q_{0}\right) \mathrm{d}_{\mathbb{S}}(q b) .
$$

Given $F \in \mathcal{B}(\mathbb{S}, A)$, this suggests to define the function

$$
F^{\eta}: \mathbb{S} \times Q \rightarrow A, \quad\left(q b, q_{0}\right) \mapsto F\left(q_{0} q b\right) \eta\left(q_{0} \underline{s}^{e} q\right)
$$

so that with $\hat{\mathbf{m}}(q b):=\mathbf{m}\left(q^{-1}\right)$ and with the notations of Proposition 7.3 , we will have

$$
\left\langle\eta_{x}, \Omega_{\theta, \mathbf{m}}(F) \eta\right\rangle_{\mathcal{H}_{x}}=\mathcal{F}^{\bar{\eta}}\left(\int_{\mathbb{S}} \mathbf{E}(y) \hat{\mathbf{m}}(y) F^{\eta}(., y) \mathrm{d}_{\mathbb{S}}(y)\right)(x) .
$$

Consequently, we obtain

$$
\begin{aligned}
& \left\langle\Phi, \Omega_{\theta, \mathbf{m}}(F) \Psi\right\rangle_{\mathcal{H}_{\chi} \otimes \mathcal{H}}= \\
& \int_{\mathbb{S} \times \mathbb{S}}\left\langle\left\langle\eta_{x}, \Psi\right\rangle_{\mathcal{H}_{\chi}}, \mathcal{F}^{\bar{\eta}}\left(\int_{\mathbb{S}} \mathbf{E}(z) \hat{\mathbf{m}}(z)\left(L_{y^{-1}}^{\star} F\right)^{\eta}(., z) \mathrm{d}_{\mathbb{S}}(z)\right)\left(y^{-1} x\right)\left\langle\eta_{y}, \Psi\right\rangle_{\mathcal{H}_{\chi}}\right\rangle_{\mathcal{H}} \\
& \times \mathrm{d}_{\mathbb{S}}(x) \mathrm{d}_{\mathbb{S}}(y) .
\end{aligned}
$$

Surprisingly, this is the right hand side of the (formal) equality above which gives rise to a well defined and bounded quadratic form on $\mathcal{H}_{\chi} \otimes \mathcal{H}$, once the integral sign in the middle is replaced by an oscillatory one in the sense of Theorem 7.17 for the tempered pair $(\mathbb{S}, \mathbf{S})$.

Coming back to the case of a generic normal $\mathbf{j}$-group $\mathbb{B}$, the most important step is to understand the properties of the corresponding map $F \mapsto F^{\eta}$ given in (7.14).

Lemma 7.18. Let $A$ be a $C^{*}$-algebra, $\mathbb{B}$ be a normal $\mathbf{j}$-group with PyatetskiiShapiro decomposition $\mathbb{B}=\left(\mathbb{S}_{N} \ltimes \ldots\right) \ltimes \mathbb{S}_{1}$ and $\eta \in \mathcal{D}\left(Q_{N} \times \cdots \times Q_{1}\right)$. Then the map

$$
\begin{aligned}
F \mapsto F^{\eta}:= & {\left[q _ { N } b _ { N } \in \mathbb { S } _ { N } \mapsto \left[q _ { N - 1 } b _ { N - 1 } \in \mathbb { S } _ { N - 1 } \mapsto \ldots \left[q_{1} b_{1} \in \mathbb{S}_{1} \mapsto\right.\right.\right.} \\
{\left[\left(q_{N}^{\prime}, \ldots, q_{1}^{\prime}\right) \in Q_{N} \times \cdots \times Q_{1} \mapsto\right.} & F\left(q_{1}^{\prime} q_{1} b_{1} \ldots q_{N-1}^{\prime} q_{N-1} b_{N-1} q_{N}^{\prime} q_{N} b_{N}\right) \\
& \left.\left.\left.\left.\times \eta\left(q_{N}^{\prime} \underline{s}^{e} q_{N}, \ldots, q_{1}^{\prime} \underline{s}^{e} q_{1}\right) \in A\right]\right] \ldots\right]\right],
\end{aligned}
$$

\footnotetext{
${ }^{4}$ Observe that this property holds for $F \in \mathcal{S}^{S_{\text {can }}^{\mathbb{B}}}(\mathbb{B}, A)$, by Proposition 6.29 as $\mathcal{S}^{S_{\text {can }}^{\mathbb{B}}}(\mathbb{B}, A) \subset$ $\mathcal{S}(\mathbb{B} ; A)$.
} 
is continuous from $\mathcal{B}(\mathbb{B}, A)$ to

$$
\mathcal{B}^{\underline{\mu}}{ }^{, \nu_{N}}\left(\mathbb{S}_{N}, \mathcal{B}^{\underline{\mu}_{N-1}, \nu_{N-1}}\left(\mathbb{S}_{N-1}, \ldots \mathcal{B}^{\mu_{1}, \nu_{1}}\left(\mathbb{S}_{1}, \mathcal{S}\left(Q_{N} \times \cdots \times Q_{1}, A\right)\right) \ldots\right)\right),
$$

where for $j=N, \ldots 1$, we have settled:

$$
\nu_{j}:=\mathfrak{d}_{Q_{j}}^{2}, \quad \underline{\mu}_{j}:=\left\{\mathfrak{d}_{\mathbb{S}_{j}}^{n_{j}\left(k_{j-1}, l_{j-1} ; \ldots ; k_{1}, l_{1} ; k, l\right)}\right\}_{\left(k_{j-1}, l_{j-1} ; \ldots ; k_{1}, l_{1} ; k, l\right) \in \mathbb{N}^{2 j}},
$$

where $\left(k_{j-1}, l_{j-1} ; \ldots ; k_{1}, l_{1} ; k, l\right) \in \mathbb{N}^{2 j}$ labels the semi-norms of the space

$$
\mathcal{B}^{\underline{\mu}_{j-1}, \nu_{j-1}}\left(\mathbb{S}_{j-1}, \ldots \mathcal{B}^{\underline{\mu}}, \nu_{1}\left(\mathbb{S}_{1}, \mathcal{S}\left(Q_{N} \times \cdots \times Q_{1}, A\right)\right) \ldots\right),
$$

and the exponent $n_{j}\left(k_{j-1}, l_{j-1} ; \ldots ; k_{1}, l_{1} ; k, l\right) \in \mathbb{N}$ is linear in its arguments.

Proof. For notational convenience, we assume that $\mathbb{B}$ contains only two elementary factors, i.e. $\mathbb{B}=\mathbb{S}_{2} \ltimes \mathbb{S}_{1}$ with $\mathbb{S}_{1}, \mathbb{S}_{2}$ elementary normal $\mathbf{j}$-groups. This is enough to understand the global mechanism and the proof for a generic normal j-group with an arbitrary number of elementary factors will then follow by induction, without essential supplementary difficulties. In this simplified case, we have to prove that the map

$$
\begin{aligned}
F \mapsto F^{\eta}:=\left[q _ { 2 } b _ { 2 } \in \mathbb { S } _ { 2 } \mapsto \left[q_{1} b_{1}\right.\right. & \in \mathbb{S}_{1} \mapsto\left[\left(q_{2}^{\prime}, q_{1}^{\prime}\right) \in Q_{2} \times Q_{1} \mapsto\right. \\
& \left.\left.\left.F\left(q_{1}^{\prime} q_{1} b_{1} q_{2}^{\prime} q_{2} b_{2}\right) \eta\left(q_{2}^{\prime} \underline{s}^{e} q_{2}, q_{1}^{\prime} \underline{s}^{e} q_{1}\right) \in A\right]\right]\right],
\end{aligned}
$$

is continuous from $\mathcal{B}(\mathbb{B}, A)$ to

$$
\mathcal{B}^{\underline{\mu}}{ }^{2}, \nu_{2}\left(\mathbb{S}_{2}, \mathcal{B}^{\mu_{1}}, \nu_{1}\left(\mathbb{S}_{1}, \mathcal{S}\left(Q_{2} \times Q_{1}, A\right)\right) .\right.
$$

By the discussion following Remark 6.12, it is clear that one may regard $\mathcal{S}\left(Q_{2} \times\right.$ $\left.Q_{1}, A\right)$ as a Fréchet space for the topology induced by the following countable set of semi-norms:

$$
\|f\|_{k, j}:=\sup _{Z^{2} \in \mathcal{U}_{k}\left(\mathfrak{q}_{2}\right)} \sup _{Z^{1} \in \mathcal{U}_{k}\left(\mathfrak{q}_{1}\right)} \sup _{\left(q_{2}, q_{1}\right) \in Q_{2} \times Q_{1}}\left\{\frac{\mathfrak{d}_{Q_{2}}\left(q_{2}\right)^{j} \mathfrak{d}_{Q_{1}}\left(q_{1}\right)^{j}\left\|\underline{Z}_{q_{2}}^{2} \underline{Z}_{q_{1}}^{1} f\left(q_{1}, q_{2}\right)\right\|}{\left|Z^{2}\right|_{k}\left|Z^{1}\right|_{k}}\right\},
$$

i.e. we may use right-invariant vector fields instead of left-invariant one since they are related by tempered functions with tempered inverses. Note then that the natural Fréchet topology of the space (7.15) is associated with the following countable family of semi-norms (indexed by $\left.\left(k_{2}, l_{2}, k_{1}, l_{1}, k, j\right) \in \mathbb{N}^{6}\right)$ :

$$
\begin{aligned}
& \Phi \mapsto \sup _{X \in \mathcal{U}_{k_{2}}\left(\mathfrak{q}_{2}\right) X^{\prime} \in \mathcal{U}_{l_{2}}\left(\mathfrak{Y}_{2}\right)} \sup _{q_{2} b_{2} \in \mathbb{S}_{2} Y \in \mathcal{U}_{k_{1}}\left(\mathfrak{q}_{1}\right) Y^{\prime} \in \operatorname{Uup}_{l_{1}}\left(\mathfrak{Y}_{1}\right)} \sup _{q_{1} b_{1} \in \mathbb{S}_{1}} \sup _{Z \in \mathcal{U}_{k}\left(\mathfrak{q}_{2} \oplus \mathfrak{q}_{1}\right)\left(q_{2}, q_{1}\right) \in Q_{2} \times Q_{1}} \sup _{Q_{1}} \\
& \frac{\mathfrak{d}_{Q_{2}}\left(q_{2}^{\prime}\right)^{j} \mathfrak{d}_{Q_{1}}\left(q_{1}^{\prime}\right)^{j}\left\|\underline{Z}_{\left(q_{2}^{\prime}, q_{1}^{\prime}\right)} \widetilde{Y}_{q_{1} b_{1}} \widetilde{Y}_{q_{1} b_{1}}^{\prime} \widetilde{X}_{q_{2} b_{2}} \widetilde{X}_{q_{2} b_{2}}^{\prime} \Phi\left(q_{2} b_{2} ; q_{1} b_{1} ; q_{2}^{\prime}, q_{1}^{\prime}\right)\right\|}{\mathfrak{d}_{\mathbb{S}_{2}}\left(q_{2} b_{2}\right)^{n_{2}\left(k_{1}, l_{1}, k, j\right)} \mathfrak{d}_{Q_{2}}\left(q_{2}\right)^{2 k_{2}} \mathfrak{d}_{\mathbb{S}_{1}}\left(q_{1} b_{1}\right)^{n_{1}(k, j)} \mathfrak{d}_{Q_{1}}\left(q_{1}\right)^{2 k_{1}}|X|_{k_{2}}\left|X^{\prime}\right| l_{2}|Y|_{k_{1}}\left|Y^{\prime}\right| l_{1}|Z|_{k}} .
\end{aligned}
$$

To simplify the notations, we denote the latter semi-norm by $\|\cdot\|_{k_{2}, l_{2}, k_{1}, l_{1}, k, j}$. Then, for

$$
\left(X, X^{\prime}, Y, Y^{\prime}, Z^{2}, Z^{1}\right) \in \mathcal{U}\left(\mathfrak{q}_{2}\right) \times \mathcal{U}\left(\mathfrak{Y}_{2}\right) \times \mathcal{U}\left(\mathfrak{q}_{1}\right) \times \mathcal{U}\left(\mathfrak{Y}_{1}\right) \times \mathcal{U}\left(\mathfrak{q}_{2}\right) \times \mathcal{U}\left(\mathfrak{q}_{1}\right),
$$

we get within Sweedler's notation:

$$
\begin{gathered}
\underline{Z}_{q_{2}^{\prime}}^{2} \underline{Z}_{q_{1}^{\prime}}^{1} \widetilde{Y}_{q_{1} b_{1}} \tilde{Y}_{q_{1} b_{1}}^{\prime} \widetilde{X}_{q_{2} b_{2}} \tilde{X}_{q_{2} b_{2}}^{\prime} F^{\eta}\left(q_{1} b_{1} ; q_{2} b_{2} ; q_{2}^{\prime}, q_{1}^{\prime}\right)=\sum_{(X)} \sum_{\left(X^{\prime}\right)} \sum_{(Y)} \sum_{\left(X^{\prime}\right)\left(Z^{2}\right)} \sum_{\left(Z^{1}\right)} \\
\left(\underline{Z}_{(1) q_{2}^{\prime}}^{2} \underline{Z}_{(1) q_{1}^{\prime}}^{1} \widetilde{Y}_{(1) q_{1} b_{1}} \widetilde{Y}_{(1) q_{1} b_{1}}^{\prime} \widetilde{X}_{(1) q_{2} b_{2}} \widetilde{X}_{(1) q_{2} b_{2}}^{\prime} F\left(q_{1}^{\prime} q_{1} b_{1} q_{2}^{\prime} q_{2} b_{2}\right)\right) \\
\times\left(\underline{Z}_{(2) q_{2}^{\prime}}^{2} \underline{Z}_{(2) q_{1}^{\prime}}^{1} \widetilde{Y}_{(2) q_{1}} \widetilde{X}_{(2) q_{2}} \eta\left(q_{2}^{\prime} \underline{s}^{e}\left(q_{2}\right), q_{1}^{\prime} \underline{s}^{e}\left(q_{1}\right)\right)\right) .
\end{gathered}
$$


From the same reasoning as those in the proof of Lemma 1.8 (v), we deduce that

$$
\begin{aligned}
\left\|F^{\eta}\right\|_{k_{2}, k_{1}, k, j} \leq & C \sup \frac{\mathfrak{d}_{Q_{2}}\left(q_{2}^{\prime}\right)^{j} \mathfrak{d}_{Q_{1}}\left(q_{1}^{\prime}\right)^{j}}{\mathfrak{d}_{\mathbb{S}_{2}}\left(q_{2} b_{2}\right)^{n_{2}\left(k_{1}, l_{1}, k, j\right)} \mathfrak{d}_{Q_{2}}\left(q_{2}\right)^{2 k_{2}} \mathfrak{d}_{\mathbb{S}_{1}}\left(q_{1} b_{1}\right)^{n_{1}(k, j)} \mathfrak{d}_{Q_{1}}\left(q_{1}\right)^{2 k_{1}}} \\
& \times \sup \frac{\left\|\underline{Z}_{q_{1}^{\prime}}^{1} \underline{Z}_{q_{2}^{2}}^{2} \widetilde{Y}_{q_{1} b_{1}} \widetilde{Y}_{q_{1} b_{1}}^{\prime} \widetilde{X}_{q_{2} b_{2}} \widetilde{X}_{q_{2} b_{2}}^{\prime} F\left(q_{1}^{\prime} q_{1} b_{1} q_{2}^{\prime} q_{2} b_{2}\right)\right\|}{|X|_{k_{2}}\left|X^{\prime}\right|_{l_{2}}|Y|_{k_{1}}\left|Y^{\prime}\right|_{l_{1}}\left|Z^{2}\right|_{k}\left|Z^{1}\right|_{k}} \\
& \times \sup \frac{\left|\underline{Z}_{q_{1}^{\prime}}^{1} \underline{Z}_{q_{2}^{\prime}}^{2} \widetilde{Y}_{q_{1}} \widetilde{X}_{q_{2}} \eta\left(q_{2}^{\prime} \underline{s}^{e}\left(q_{2}\right), q_{1}^{\prime} \underline{s}^{e}\left(q_{1}\right)\right)\right|}{|X|_{k_{2}}|Y|_{k_{1}}\left|Z^{2}\right|_{k}\left|Z^{1}\right|_{k}},
\end{aligned}
$$

where the first supremum is over:

$$
\left(q_{2} b_{2}, q_{1} b_{1}, q_{2}^{\prime}, q_{1}^{\prime}\right) \in \mathbb{S}_{2} \times \mathbb{S}_{1} \times Q_{2} \times Q_{1},
$$

the second over:

$$
\left(X, X^{\prime}, Y, Y^{\prime}, Z^{2}, Z^{1}\right) \in \mathcal{U}_{k_{2}}\left(\mathfrak{q}_{2}\right) \times \mathcal{U}_{l_{2}}\left(\mathfrak{Y}_{2}\right) \times \mathcal{U}_{k_{1}}\left(\mathfrak{q}_{1}\right) \times \mathcal{U}_{l_{1}}\left(\mathfrak{Y}_{1}\right) \times \mathcal{U}_{k}\left(\mathfrak{q}_{2}\right) \times \mathcal{U}_{k}\left(\mathfrak{q}_{1}\right),
$$

and the third over:

$$
\left(X, Y, Z^{2}, Z^{1}\right) \in \mathcal{U}_{k_{2}}\left(\mathfrak{q}_{2}\right) \times \mathcal{U}_{k_{1}}\left(\mathfrak{q}_{1}\right) \times \mathcal{U}_{k}\left(\mathfrak{q}_{2}\right) \times \mathcal{U}_{k}\left(\mathfrak{q}_{1}\right) .
$$

Next, we observe:

$$
\begin{aligned}
& \underline{Z}_{q_{1}^{\prime}}^{1} \underline{Z}_{q_{2}}^{2} \widetilde{Y}_{q_{1} b_{1}} \tilde{Y}_{q_{1} b_{1}}^{\prime} \widetilde{X}_{q_{2} b_{2}} \tilde{X}_{q_{2} b_{2}}^{\prime} F\left(q_{1}^{\prime} q_{1} b_{1} q_{2}^{\prime} q_{2} b_{2}\right)= \\
& \left(\operatorname{Ad}_{\left(q_{1}^{\prime} q_{1} b_{1} q_{2}^{\prime} q_{2} b_{2}\right)^{-1}}\left(Z^{1}\right) \operatorname{Ad}_{\left(q_{2}^{\prime} q_{2} b_{2}\right)^{-1}}\left(Z^{2}\right) \operatorname{Ad}_{\left(q_{2}^{\prime} q_{2} b_{2}\right)^{-1}}\left(Y Y^{\prime}\right) \tilde{X} \tilde{X}^{\prime} F\right)\left(q_{1}^{\prime} q_{1} b_{1} q_{2}^{\prime} q_{2} b_{2}\right) .
\end{aligned}
$$

This, together with Lemma 1.14, entails that the $F$-dependent supremum in (7.16) is, up to a constant, bounded by:

$$
\|F\|_{2 k+k_{1}+l_{1}+k_{2}+l_{2}} \mathfrak{d}_{\mathbb{S}_{2} \ltimes \mathbb{S}_{1}}\left(q_{1}^{\prime} q_{1} b_{1} q_{2}^{\prime} q_{2} b_{2}\right)^{k} \mathfrak{d}_{\mathbb{S}_{2} \ltimes \mathbb{S}_{1}}\left(q_{2}^{\prime} q_{2} b_{2}\right)^{k+k_{1}+l_{1}},
$$

which by sub-multiplicativity of the modular weight, is bounded by:

$$
\|F\|_{2 k+k_{1}+l_{1}+k_{2}+l_{2}} \mathfrak{d}_{\mathbb{S}_{2} \ltimes \mathbb{S}_{1}}\left(q_{2}^{\prime} q_{2} b_{2}\right)^{2 k+k_{1}+l_{1}} \mathfrak{d}_{\mathbb{S}_{2} \ltimes \mathbb{S}_{1}}\left(q_{1}^{\prime} q_{1} b_{1}\right)^{k} .
$$

Now, by Theorem 2.35] the pair $\left(\left(\mathbb{S}_{2} \ltimes \mathbb{S}_{1}\right)^{2}, S_{\text {can }}^{\mathbb{S}_{1}} \oplus 1+1 \oplus S_{\text {can }}^{\mathbb{S}_{1}}\right)$ is tempered (and admissible and tame), so that by Lemma 1.21 the modular weight $\mathfrak{d}_{\left(\mathbb{S}_{2} \ltimes \mathbb{S}_{1}\right)^{2}}$ is tempered. Then, using the last statement of Lemma 1.5, together with the methods of Lemmas 2.33 and 2.34 we see that $\mathfrak{d}_{\mathbb{S}_{2} \ltimes \mathbb{S}_{1}}$ is tempered in (any) adapted coordinates (see Definition 2.32) for $\mathbb{B}=\mathbb{S}_{2} \ltimes \mathbb{S}_{1}$. This clearly implies that the restriction $\mathfrak{d}_{\mathbb{S}_{2} \ltimes \mathbb{S}_{1}} \mid \mathbb{S}_{j}, j=1,2$, is also tempered in the adapted coordinates for $\mathbb{S}_{j}$. In view of the expressions (7.10), we see that the adapted tempered coordinates and the coordinates associated to the one-point pair $\left(\mathbb{S}_{j}, \mathbf{S}_{\mathbb{S}_{j}}\right)$ (which is tempered by Theorem (7.13) are related to one another through a tempered diffeomorphism. Hence, we deduce that $\mathfrak{d}_{\mathbb{S}_{2} \ltimes \mathbb{S}_{1}} \mid \mathbb{S}_{j}$ is tempered in the sense of the one-point phase function too. Last, using the explicit expression of the tempered weight $\mathfrak{d}_{\mathbb{S}_{j}}$ given in Lemma 2.27, we deduce that there exist $m_{j} \in \mathbb{N}$ and $C_{j}>0$, such that $\left.\mathfrak{d}_{\mathbb{S}_{2} \ltimes \mathbb{S}_{1}}\right|_{\mathbb{S}_{j}} \leq$ $C_{j} \mathfrak{d}_{\mathbb{S}_{j}}^{m_{j}}, j=1,2$. Hence, the $F$-dependent supremum in (7.16) is, up to a constant, bounded by

$$
\|F\|_{2 k+k_{1}+l_{1}+k_{2}+l_{2}} \mathfrak{d}_{\mathbb{S}_{2}}\left(q_{2}^{\prime} q_{2} b_{2}\right)^{m_{2}\left(2 k+k_{1}+l_{1}\right)} \mathfrak{d}_{\mathbb{S}_{1}}\left(q_{1}^{\prime} q_{1} b_{1}\right)^{m_{1} k} .
$$

For $\eta$-dependent term in (7.16), we first note:

$$
\underline{Z}_{q_{1}^{\prime}}^{1} \underline{Z}_{q_{2}^{\prime}}^{2} \widetilde{Y}_{q_{1}} \widetilde{X}_{q_{2}} \eta\left(q_{2}^{\prime} \underline{s}^{e}\left(q_{2}\right), q_{1}^{\prime} \underline{s}^{e}\left(q_{1}\right)\right)=\widetilde{Y}_{q_{1}} \widetilde{X}_{q_{2}}\left(\underline{Z}^{1} \underline{Z}^{2} \eta\right)\left(q_{2}^{\prime} \underline{s}^{e}\left(q_{2}\right), q_{1}^{\prime} \underline{s}^{e}\left(q_{1}\right)\right)
$$


so that up to a redefinition of $\eta$, we can ignore the right-invariant vector fields. Next, we observe that with $q=(a, n), q^{\prime}=\left(a^{\prime}, n^{\prime}\right)$ in the coordinates (6.10), we have:

$$
q^{\prime} \underline{s}^{e}(q)=\left(2 a+a^{\prime}, e^{-2 a} n^{\prime}+2 n \cosh a\right) .
$$

With $H$ the generator of $\mathfrak{a}$ and $\left\{f_{j}\right\}_{j=1}^{d}$ a basis of $\mathfrak{l}^{\star}$, the associated left-invariant vector fields on $Q$ read:

$$
\widetilde{H}=\partial_{a}-\sum_{j=1}^{d} n_{j} \partial_{n_{j}}, \quad \tilde{f}_{j}=\partial_{n_{j}}
$$

Choosing $\eta=\eta_{2} \otimes \eta_{1}$ with $\eta_{j} \in \mathcal{D}\left(Q_{j}\right)$, it is enough to treat each variable separately. So just assume that $\eta \in \mathcal{D}(Q)$. Now, for $N=\left(N_{1}, \ldots, N_{d}\right) \in \mathbb{N}^{d}$ with $|N|=k$, we have with $\widetilde{f}^{N}:=\widetilde{f}_{1}^{N_{1}} \ldots \widetilde{f}_{d}^{N_{d}}, \partial_{n}^{N}:=\partial_{n_{1}}^{N_{1}} \ldots \partial_{n_{d}}^{N_{d}}$ and setting $q_{0}:=q^{\prime} \underline{s}^{e}(q) \in Q$ :

$$
\widetilde{f}_{q}^{N} \eta\left(q_{0}\right)=2^{k} \cosh ^{k} a\left(\partial_{n}^{N} \eta\right)\left(q_{0}\right)
$$

Since $\cosh a \leq 2 \cosh a^{\prime} / 2 \cosh a_{0} / 2$, the latter and Remark 6.12 entail that

$$
\begin{aligned}
\left|\widetilde{f}_{q}^{N} \eta\left(q_{0}\right)\right| & \leq C \cosh \left(a^{\prime} / 2\right)^{k} \cosh \left(a_{0} / 2\right)^{k}\left|\partial_{n}^{N} \eta\right|\left(q_{0}\right) \\
& \leq C \mathfrak{d}_{Q}\left(q^{\prime}\right)^{k} \cosh \left(a_{0} / 2\right)^{k}\left|\partial_{n}^{N} \eta\right|\left(q_{0}\right) .
\end{aligned}
$$

On the other hand, we have

$$
\widetilde{H}_{q} \eta\left(q_{0}\right)=2\left(\partial_{a} \eta\right)\left(q_{0}\right)-2 \sum_{j=1}^{d}\left(n_{j} e^{-a}+n_{j}^{\prime} e^{-2 a}\right)\left(\partial_{n_{j}} \eta\right)\left(q_{0}\right)
$$

Since $w_{j}:=n_{j} e^{-a}+n_{j}^{\prime} e^{-2 a}$ is an eigenvector of $\widetilde{H}_{q}$ with eigenvalue -2 , we deduce that for $k \in \mathbb{N}, \widetilde{H}_{q}^{k} \eta\left(q_{0}\right)$ is a linear combinations of the ordinary derivatives of $\eta$, with coefficients given in the ring $\mathbb{C}\left[w_{j}\right]$ of order at most $k$. Moreover, the rough estimate:

$$
|w|=\left|\left(w_{1}, \ldots, w_{d}\right)\right| \leq 4 \cosh a_{0} \cosh a^{\prime}\left(\left|n_{0}\right|+\left|n^{\prime}\right|\right),
$$

gives by Remark 6.12 .

$$
\left|\widetilde{H}_{q}^{k} \eta\left(q_{0}\right)\right| \leq C \mathfrak{d}_{Q}\left(q^{\prime}\right)^{k} \cosh ^{k} a_{0}\left|n_{0}\right|^{k}\left|P\left(\partial_{a}, \partial_{n_{j}}\right) \eta\right|\left(q_{0}\right),
$$

for a suitable polynomial $P$. This implies that the $\eta$-dependent term in (7.16) is, up to a constant, bounded by:

$$
\mathfrak{d}_{Q_{2}}\left(q_{2}^{\prime}\right)^{k_{2}} \mathfrak{d}_{Q_{1}}\left(q_{1}^{\prime}\right)^{k_{1}}|\tilde{\eta}|\left(q_{2}^{\prime} \underline{s}^{e}\left(q_{2}\right), q_{1}^{\prime} \underline{s}^{e}\left(q_{1}\right)\right),
$$

where $\tilde{\eta}$ belongs to $\mathcal{D}\left(Q_{2} \times Q_{1}\right)$ and is obtained from $\eta$ by multiplication by $\cosh a,|n|$ and by differentiation along all its variables. Finally, we deduce (with $m_{1}, m_{2} \in \mathbb{N}$ fixed) that

$$
\begin{aligned}
& \left\|F^{\eta}\right\|_{k_{2}, l_{2}, k_{1}, l_{1}, k, j} \leq C\|F\|_{2 k+k_{1}+l_{1}+k_{2}+l_{2}} \sup _{\left(q_{2} b_{2}, q_{1} b_{1}, q_{2}^{\prime}, q_{1}^{\prime}\right) \in \mathbb{S}_{2} \times \mathbb{S}_{1} \times Q_{2} \times Q_{1}} \\
& \frac{\mathfrak{d}_{Q_{2}}\left(q_{2}^{\prime}\right)^{j+k_{2}} \mathfrak{d}_{Q_{1}}\left(q_{1}^{\prime}\right)^{j+k_{1}} \mathfrak{d}_{\mathbb{S}_{2}}\left(q_{2}^{\prime} q_{2} b_{2}\right)^{m_{2}\left(2 k+k_{1}+l_{1}\right)_{\mathfrak{d}_{1}}\left(q_{1}^{\prime} q_{1} b_{1}\right)^{m_{1}} k \mid}|\tilde{\eta}|\left(q_{2}^{\prime} \underline{s}^{e}\left(q_{2}\right), q_{1}^{\prime} \underline{s}^{e}\left(q_{1}\right)\right)}{\mathfrak{d}_{\mathbb{S}_{2}}\left(q_{2} b_{2}\right)^{n_{2}\left(k_{1}, l_{1}, k, j\right)} \mathfrak{d}_{Q_{2}}\left(q_{2}\right)^{2 k_{2}} \mathfrak{d}_{\mathbb{S}_{1}}\left(q_{1} b_{1}\right)^{n_{1}(k, j)} \mathfrak{d}_{Q_{1}}\left(q_{1}\right)^{2 k_{1}}} .
\end{aligned}
$$

Observe then that by Lemma 2.27 and Remark6.12, we have $\left.\mathfrak{d}_{\mathbb{S}}\right|_{Q} \leq C \mathfrak{d}_{Q}^{2}$. Then, by the sub-multiplicativity and the invariance under the inversion map of the modular 
weights, we deduce that the fraction above is smaller than (a constant times):

$$
\begin{aligned}
& \frac{\mathfrak{d}_{Q_{2}}\left(\underline{s}^{e}\left(q_{2}\right)\right)^{j+k_{2}+2 m_{2}\left(2 k+k_{1}+l_{1}\right)} \mathfrak{d}_{Q_{1}}\left(\underline{s}^{e}\left(q_{1}\right)\right)^{j+k_{1}+2 m_{1} k} \mathfrak{d}_{\mathbb{S}_{2}}\left(q_{2} b_{2}\right)^{m_{2}\left(2 k+k_{1}+l_{1}\right)}}{\mathfrak{d}_{\mathbb{S}_{2}}\left(q_{2} b_{2}\right)^{n_{2}\left(k_{1}, l_{1}, k, j\right)} \mathfrak{d}_{Q_{2}}\left(q_{2}\right)^{2 k_{2}} \mathfrak{d}_{\mathbb{S}_{1}}\left(q_{1} b_{1}\right)^{n_{1}(k, j)} \mathfrak{d}_{Q_{1}}\left(q_{1}\right)^{2 k_{1}}} \\
& \times \mathfrak{d}_{\mathbb{S}_{1}}\left(q_{1} b_{1}\right)^{m_{1} k} \mathfrak{d}_{Q_{2}}\left(q_{2}^{\prime} \underline{s}^{e}\left(q_{2}\right)\right)^{j+k+2 m_{2}\left(2 k+k_{1}+l_{1}\right)} \mathfrak{d}_{Q_{1}}\left(q_{1}^{\prime} \underline{s}^{e}\left(q_{1}\right)\right)^{j+k_{1}+2 m_{1} k} \\
& \times|\tilde{\eta}|\left(q_{2}^{\prime} \underline{s}^{e}\left(q_{2}\right), q_{1}^{\prime} \underline{s}^{e}\left(q_{1}\right)\right) .
\end{aligned}
$$

Because $\tilde{\eta}$ is compactly supported, the expression in the last line above is smaller than a constant. Also, since $\underline{s}^{e}(a, n)=(2 a, 2 n \cosh a)$, we deduce, by Remark 6.12 again, that $\mathfrak{d}_{Q} \circ \underline{s}^{e} \leq C \mathfrak{d}_{Q}^{2}$. Thus, the expression above is bounded by (a constant times):

$$
\frac{\mathfrak{d}_{Q_{2}}\left(q_{2}\right)^{2 j+4 m_{2}\left(2 k+k_{1}+l_{1}\right)} \mathfrak{d}_{Q_{1}}\left(q_{1}\right)^{2 j+4 m_{1} k} \mathfrak{d}_{\mathbb{S}_{2}}\left(q_{2} b_{2}\right)^{m_{2}\left(2 k+k_{1}+l_{1}\right)} \mathfrak{d}_{\mathbb{S}_{1}}\left(q_{1} b_{1}\right)^{m_{1} k}}{\mathfrak{d}_{\mathbb{S}_{2}}\left(q_{2} b_{2}\right)^{n_{2}\left(k_{1}, l_{1}, k, j\right)}},
$$

and one concludes using Lemma 2.27 and Remark 6.12, which show that for all $q \in Q, b \in \mathbb{Y}$, we have $\mathfrak{d}_{Q}(q) \leq \mathfrak{d}_{\mathbb{S}}(q b)$ and, by suitably choosing $n_{1}(k, j)$ and $n_{2}\left(k_{1}, l_{1}, k, j\right)$.

REMARK 7.19. Lemma (7.18) admits a straightforward generalization for symbols valued in a Fréchet algebra. Namely, if $\mathcal{E}$ is a Fréchet algebra and $\mu$ is a family of tempered weights on $\mathbb{B}$, then the map $F \mapsto F^{\eta}$ is continuous from $\overline{\mathcal{B}} \underline{\mu}(\mathbb{B}, \mathcal{E})$ to

$$
\mathcal{B}^{\underline{\mu}}{ }_{N}^{, \nu_{N}}\left(\mathbb{S}_{N}, \mathcal{B}^{\underline{\mu}_{N-1}, \nu_{N-1}}\left(\mathbb{S}_{N-1}, \ldots \mathcal{B}^{\mu_{1}, \nu_{1}}\left(\mathbb{S}_{1}, \mathcal{S}\left(Q_{N} \times \cdots \times Q_{1}, A\right)\right) \ldots\right)\right),
$$

where the $\underline{\mu}_{j}$ 's now depend also of the restriction of $\underline{\mu}$ to $\mathbb{S}_{j}$ (and the $\nu_{j}$ 's are unchanged).

We are now ready to prove a non-Abelian (curved) and $C^{*}$-valued version of the Calderón-Vaillancourt estimate, the main result of this chapter.

TheOREM 7.20. Let $\mathbb{B}$ be a normal $\mathbf{j}$-group, $A$ a $C^{*}$-algebra faithfully represented on a separable Hilbert space $\mathcal{H}, F \in \mathcal{B}(\mathbb{B}, A), \eta \in \mathcal{D}\left(Q_{N} \times \cdots \times Q_{1}\right)$ and $\mathbf{m} \in \mathbf{\Theta}(\mathbb{B})$. Define for $x, y \in \mathbb{B}$, the element of $A$ given by

$$
\begin{aligned}
\left\langle\eta_{x}, \Omega_{\theta, \mathbf{m}}(F) \eta_{y}\right\rangle_{\mathcal{H}_{x}}:=\mathcal{F}^{\bar{\eta}}\left(\widetilde { \mathbf { E } _ { \theta } ^ { \mathbb { S } _ { 1 } } } \hat { \mathbf { m } } _ { 1 } \ldots \left(\int _ { \mathbb { S } _ { N } } \widetilde { \mathbf { E } _ { \theta } ^ { \mathbb { S } _ { N } } } \hat { \mathbf { m } } _ { N } \left[g_{N} \in \mathbb{S}_{N} \mapsto \ldots\right.\right.\right. \\
{\left.\left.\left.\left[g_{1} \in \mathbb{S}_{1} \mapsto\left(L_{y^{-1}}^{\star} F\right)^{\eta}\left(g_{N}, \ldots, g_{1} ; .\right)\right] \ldots\right]\right) \ldots\right)\left(y^{-1} x\right), }
\end{aligned}
$$

where $\hat{\mathbf{m}}(q b)=\mathbf{m}\left(q^{-1}\right)$ and where $\mathbf{E}_{\theta}^{\mathbb{S}_{j}}$ is the one-point phase of $\mathbb{S}_{j}$ as defined in (7.9). Then we have:

$\sup _{x \in \mathbb{B}} \int_{\mathbb{B}}\left\|\left\langle\eta_{x}, \Omega_{\theta, \mathbf{m}}(F) \eta_{y}\right\rangle_{\mathcal{H}_{\chi}}\right\| \mathrm{d}_{\mathbb{B}}(y)<\infty, \sup _{y \in \mathbb{B}} \int_{\mathbb{B}}\left\|\left\langle\eta_{x}, \Omega_{\theta, \mathbf{m}}(F) \eta_{y}\right\rangle_{\mathcal{H}_{\chi}}\right\| \mathrm{d}_{\mathbb{B}}(x)<\infty$.

Consequently (see Proposition 7.9), the operator $\Omega_{\theta, \mathbf{m}}(F)$ on $\mathcal{H}_{\chi} \otimes \mathcal{H}$ defined by means of the quadratic form

$$
\Psi, \Phi \in \mathcal{H}_{\chi} \otimes \mathcal{H} \mapsto \int_{\mathbb{B} \times \mathbb{B}}\left\langle\left\langle\eta_{x}, \Psi\right\rangle_{\mathcal{H}_{\chi}},\left\langle\eta_{x}, \Omega_{\theta, \mathbf{m}}(F) \eta_{y}\right\rangle_{\mathcal{H}_{\chi}}\left\langle\eta_{y}, \Psi\right\rangle_{\mathcal{H}_{\chi}}\right\rangle_{\mathcal{H}} \mathrm{d}_{\mathbb{B}}(x) \mathrm{d}_{\mathbb{B}}(y),
$$

is bounded. Moreover, there exists $k \in \mathbb{N}$ (depending only on $\operatorname{dim} \mathbb{B}$ and on the order of the polynomial in $\mathfrak{d}_{Q_{N}} \otimes \cdots \otimes \mathfrak{d}_{Q_{1}}$ that majorizes $|\mathbf{m}|$ ) and $C>0$, such 
that for all $F \in \mathcal{B}(\mathbb{B}, A)$ we have

$$
\left\|\Omega_{\theta, \mathbf{m}}(F)\right\| \leq C\|F\|_{k, \infty}=C \sup _{X \in \mathcal{U}_{k}(\mathfrak{b})} \sup _{x \in \mathbb{B}}\|\widetilde{X} F(x)\| .
$$

Proof. To simplify the notation for the matrix element given in (7.17), we write

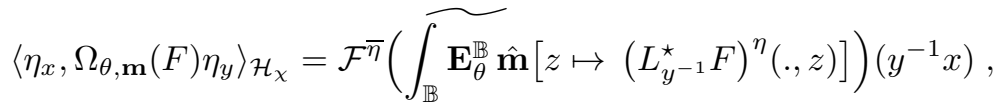

where $\mathbf{E}_{\theta}^{\mathbb{B}}$ is given in (7.11). Observe that this notation is coherent with our Fubini type Theorem 1.39. Thus,

$$
\begin{aligned}
& \sup _{y \in \mathbb{B}} \int_{\mathbb{B}}\left\|\left\langle\eta_{x}, \Omega_{\theta, \mathbf{m}}(F) \eta_{y}\right\rangle_{\mathcal{H}_{\chi}}\right\| \mathrm{d}_{\mathbb{B}}(x)
\end{aligned}
$$

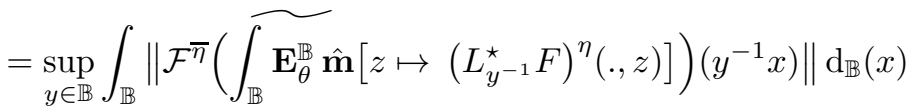

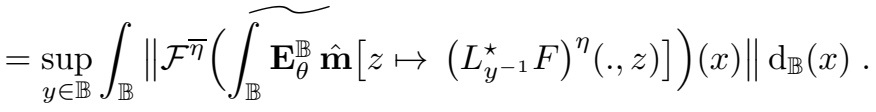

The fact that this expression is finite follows then by combining Propositions 7.3 and 7.5 with Theorem 7.17 and Lemma 7.18 and the fact that $L_{y^{-1}}^{\star}$ maps $\mathcal{B}(\mathbb{B}, A)$ to itself isometrically. The second case is similar since

$$
\left\langle\eta_{x}, \Omega_{\theta, \mathbf{m}}(F) \eta_{y}\right\rangle_{\mathcal{H}_{\chi}}^{*}=\left\langle\eta_{y}, \Omega_{\theta, \underline{\sigma^{\star}}} \overline{\mathbf{m}}\left(F^{*}\right) \eta_{x}\right\rangle_{\mathcal{H}_{\chi}} .
$$

The final estimation we give is a consequence of Proposition 7.9 together with the estimates underlying Lemma 7.5. Theorem 7.17 and Lemma 7.18

REMARK 7.21. In view of Remark 7.19, on may wonder what happens in Theorem 7.20 when one choses a symbol in $\mathcal{B}^{\mu}(\mathbb{B}, A)$ instead of a symbol in $\mathcal{B}(\mathbb{B}, A)$. So let $F \in \mathcal{B}^{\mu}(\mathbb{B}, A)$, with $\mu$ a tempered weight. Then, from the same argument than those of Theorem 7.20 (using by Remark 7.19, instead of Lemma 7.18), one deduces that

$$
\int_{\mathbb{B}}\left\|\left\langle\eta_{x}, \Omega_{\theta, \mathbf{m}}(F) \eta_{y}\right\rangle_{\mathcal{H}_{\chi}}\right\| \mathrm{d}_{\mathbb{B}}(x)<\infty .
$$

However, as the left regular action is no longer isometric on $\mathcal{B}^{\mu}(\mathbb{B}, A$ ) (see for instance the second item of Lemma 1.12), there is no reason to expect that the supremum over $y \in \mathbb{B}$ of the expression given in (7.18) to be finite. Accordingly (and as expected), there is no chance for the operator $\Omega_{\theta, \mathbf{m}}(F)$ to be bounded when $F$ belongs to $\mathcal{B}^{\mu}(\mathbb{B}, A)$ with unbounded $\mu$.

\subsection{The deformed $C^{*}$-norm}

Now, we assume that our $C^{*}$-algebra $A$ is equipped with a strongly continuous and isometric action $\alpha$ of a normal $\mathbf{j}$-group $\mathbb{B}$. We stress that the results of this chapter cannot hold true in the more general context of tempered actions. This is the main difference between the deformation theory at the level of Fréchet and $C^{*}$-algebras. Given an element $a \in A$, we construct as usual the $A$-valued function $\alpha(a)$ on $\mathbb{B}$ :

$$
\alpha(a):=\left[g \in \mathbb{B} \mapsto \alpha_{g}(a) \in A\right] .
$$


Thus, from Theorem 4.8, we can deform the Fréchet algebra structure on the set of smooth vectors $A^{\infty}$ by means of the deformed product

$$
a \star_{\theta, \mathbf{m}}^{\alpha} b:=\left(\alpha(a) \star_{\theta, \mathbf{m}} \alpha(b)\right)(e), \quad a, b \in A^{\infty} .
$$

We have seen in (6.15) how to modify the original involution at the level of $\mathcal{B}(\mathbb{B}, A)$. At the level of the Fréchet algebra $A^{\infty}$, an obvious observation leads to:

Lemma 7.22. Let $\mathbb{B}$ be a normal $\mathbf{j}$-group. For $\mathbf{m} \in \mathbf{\Theta}(\mathbb{B})$, the following defines a continuous involution of the Fréchet algebra $\left(A^{\infty}, \star_{\theta, \mathbf{m}}^{\alpha}\right)$ :

$*_{\theta, \mathbf{m}}: A^{\infty} \rightarrow A^{\infty}, \quad a \mapsto \frac{\mathbf{m}_{\mathbf{N}}}{{\underline{\underline{s_{e}^{\star}}}}_{\overline{\mathbf{m}}}}\left(\frac{1}{2} \operatorname{arcsinh}\left(\frac{i}{\theta} E_{N}^{\alpha}\right)\right) \ldots \frac{\mathbf{m}_{\mathbf{1}}}{\underline{\underline{S}}_{e}^{\star} \overline{\mathbf{m}}_{1}}\left(\frac{1}{2} \operatorname{arcsinh}\left(\frac{i}{\theta} E_{1}^{\alpha}\right)\right) a^{*}$,

where $E_{N}, \ldots, E_{1}$ are the central elements of the Heisenberg Lie algebras attached to each elementary factors of $\mathbb{B}$.

REMark 7.23. Note that when $\underline{s}_{e}^{\star} \overline{\mathbf{m}}_{j}=\mathbf{m}_{j}, j=1, \ldots, N$, there is no modification of the involution.

The construction of a pre- $C^{*}$-structure on $\left(A^{\infty}, \star_{\theta, \mathbf{m}}^{\alpha}\right)$ follows then from Theorem 7.20 and from the following immediate result (compare with Lemma 4.5):

Lemma 7.24. Let $(A, \alpha, \mathbb{B})$ be a $C^{*}$-algebra endowed with a strongly continuous and isometric action of a normal $\mathbf{j}$-group. Then, we have an isometric equivariant embedding $\alpha: A^{\infty} \rightarrow \mathcal{B}(\mathbb{B}, A)$.

Proof. The equivariance property of $\alpha$ is obvious and implies (with the fact that $\alpha$ is an isometric action of $\mathbb{B}$ on $A$ ) that for any $k \in \mathbb{N}$ :

$$
\begin{aligned}
\|\alpha(a)\|_{k, \infty} & =\sup _{g \in \mathbb{B}} \sup _{X \in \mathcal{U}_{k}(\mathfrak{b})} \frac{\left\|\widetilde{X}_{g} \alpha_{g}(a)\right\|}{|X|_{k}} \\
& =\sup _{g \in \mathbb{B}} \sup _{X \in \mathcal{U}_{k}(\mathfrak{b})} \frac{\left\|\alpha_{g}\left(X^{\alpha} a\right)\right\|}{|X|_{k}}=\sup _{X \in \mathcal{U}_{k}(\mathfrak{b})} \frac{\left\|X^{\alpha} a\right\|}{|X|_{k}}=\|a\|_{k},
\end{aligned}
$$

and the proof follows.

Recall that $\mathcal{H}$ is any separable Hilbert space carrying a faithful representation of our $C^{*}$-algebra $A$, which therefore, is identified with a $C^{*}$-subalgebra of $\mathcal{B}(\mathcal{H})$. Then by the previous lemma and Theorem 7.20, we deduce that the map

$$
a \in A^{\infty} \mapsto\left\|\Omega_{\theta, \mathbf{m}}(\alpha(a))\right\|,
$$

takes finite values. It is also important to observe that the norm above is by construction the operator norm on $\mathcal{H}_{\chi} \otimes \mathcal{H}$, that is the spatial $C^{*}$-norm on $A \otimes$ $\mathcal{B}\left(\mathcal{H}_{\chi}\right)$. For future use, we also recall that the spatial $C^{*}$-norm is the minimal $C^{*}$ cross-norm. (We invite the reader unfamiliar with tensor products of $C^{*}$-algebras to consult, for example, Appendix B of [24].) More precisely, combining the Lemma 7.24 with Theorem 7.20, we deduce the following inequality:

Corollary 7.25. Let $(A, \alpha, \mathbb{B})$ be a $C^{*}$-algebra endowed with a strongly continuous action of a normal $\mathbf{j}$-group and $\mathbf{m} \in \mathbf{\Theta}(\mathbb{B})$. Then, there exists $k \in \mathbb{N}$ and $C>0$ such that for any $a \in A^{\infty}$, we have:

$$
\left\|\Omega_{\theta, \mathbf{m}}(\alpha(a))\right\| \leq C\|a\|_{k}:=C \sup _{X \in \mathcal{U}_{k}(\mathfrak{b})}\left\{\frac{\left\|X^{\alpha} a\right\|}{|X|_{k}}\right\} .
$$


Proposition 7.26. Let $\mathbf{m} \in \mathbf{\Theta}(\mathbb{B})$. Then, the following defines a $C^{*}$-norm on the involutive deformed Fréchet algebra $\left(A^{\infty}, \star_{\theta, \mathbf{m}}^{\alpha}, *_{\theta, \mathbf{m}}\right)$ :

$$
a \in A^{\infty} \mapsto\|a\|_{\theta, \mathbf{m}}:=\left\|\Omega_{\theta, \mathbf{m}}(\alpha(a))\right\|,
$$

where the operator $\Omega_{\theta, \mathbf{m}}(\alpha(a))$ is defined in Theorem 7.20. Accordingly, we let $A_{\theta, \mathbf{m}}$ be the $C^{*}$-completion of $A^{\infty}$ that we abusively call the $C^{*}$-deformation of $A$.

Proof. By construction we have for all $a, b \in A^{\infty}$

$$
\Omega_{\theta, \mathbf{m}}\left(\alpha\left(a^{*_{\theta, \mathbf{m}}} \star_{\theta, \mathbf{m}}^{\alpha} b\right)\right)=\Omega_{\theta, \mathbf{m}}(\alpha(a))^{*} \Omega_{\theta, \mathbf{m}}(\alpha(b)),
$$

and the claim follows immediately.

REMARK 7.27. We already know that at the level of the deformed pre- $C^{*}$ algebra $\left(A^{\infty}, \star_{\theta, \mathbf{m}}^{\alpha}\right)$, the action of the group $\mathbb{B}$ is no longer by automorphism. But at the level of the deformed $C^{*}$-algebra there is no action of $\mathbb{B}$ at all.

In a way very analogous to Proposition 4.20 , we can show that the $C^{*}$-deformation associated with a normal $\mathbf{j}$-group coincides with the iterated $C^{*}$-deformations of each of its elementary normal subgroups. To see this, fix $\mathbb{B}$ be a normal $\mathbf{j}$-group with Pyatetskii-Shapiro decomposition $\mathbb{B}=\mathbb{B}^{\prime} \ltimes \mathbb{S}$ and $A$ a $C^{*}$-algebra endowed with a strongly continuous and isometric action $\alpha$ of $\mathbb{B}$. Of course, $\alpha^{\mathbb{S}}$, the restriction of $\alpha$ to the subgroup $\mathbb{S}$, is strongly continuous on $A$. Let us fix also $\tilde{\mathbf{m}}=\mathbf{m}^{\prime} \otimes \mathbf{m} \in \boldsymbol{\Theta}(\mathbb{B})$, with $\mathbf{m}^{\prime} \in \boldsymbol{\Theta}\left(\mathbb{B}^{\prime}\right)$ and $\mathbf{m} \in \boldsymbol{\Theta}(\mathbb{S})$. Then, we can perform the $C^{*}$-deformation of $A$ by means of the action of $\mathbb{S}$. We call this deformed $C^{*}$-algebra $A_{\theta, \mathbf{m}}^{\mathbb{S}}$. Then $\mathbb{B}^{\prime}$ acts strongly continuously by $*$-homomorphisms on $A_{\theta, \mathbf{m}}^{\mathbb{S}}$. Indeed, it has been shown in the proof of Proposition 4.20 that the subspace of smooth vectors for $\mathbb{B}$ coincides with the subspace of smooth vectors for $\mathbb{B}^{\prime}$ within the subspace of smooth vectors for $\mathbb{S}$. In turns, $A^{\infty}$, the set of smooth vectors for $\mathbb{B}$ on $A$, is dense in $A_{\theta, \mathbf{m}}^{\mathbb{S}}$. As the action of $\mathbb{B}^{\prime}$ is (obviously) strongly continuous and by $*$-homomorphisms (as shown in the proof of Proposition 4.20 too) on $A^{\infty}$, a density argument yields the result. Thus, we can perform the $C^{*}$-deformation of $A_{\theta, \mathbf{m}}^{\mathbb{S}}$ by means of the action of $\mathbb{B}^{\prime}$. We call this deformed $C^{*}$-algebra $\left(A_{\theta, \mathbf{m}}^{\mathbb{S}}\right)_{\theta \mathbf{m}^{\prime}}^{\mathbb{B}^{\prime}}$. But we could also perform the $C^{*}$-deformation of $A$ by means of the action of $\mathbb{B}$ directly. We call this deformed $C^{*}$-algebra $A_{\theta, \tilde{\mathbf{m}}}^{\mathbb{B}}$. Now, the precise result of Proposition 4.20, is that at the level of the (common) dense subspace $A^{\infty}$, both constructions coincide. Thus it suffices to show that the $C^{*}$-norms of $\left(A_{\theta, \mathbf{m}}^{\mathbb{S}}\right)_{\theta, \mathbf{m}^{\prime}}^{\mathbb{B}^{\prime}}$ and $A_{\theta, \tilde{\mathbf{m}}}^{\mathbb{B}}$ coincide on $A^{\infty}$. But this easily follows from our construction. Indeed, the $C^{*}$-norm of $\left(A_{\theta, \mathbf{m}}^{\mathbb{S}}\right)_{\theta, \mathbf{m}^{\prime}}^{\mathbb{B}^{\prime}}$ at the level of $A^{\infty}$, is by definition the map

$$
a \mapsto\left\|\Omega_{\theta, \mathbf{m}^{\prime}}^{\mathbb{B}^{\prime}}\left(\left[z^{\prime} \in \mathbb{B}^{\prime} \mapsto \Omega_{\theta, \mathbf{m}}^{\mathbb{S}}\left(\left[z \in \mathbb{S} \mapsto \alpha_{z z^{\prime}}(a)\right]\right)\right]\right)\right\| .
$$

But by the construction of section 6.3, we precisely have

$$
\Omega_{\theta, \mathbf{m}^{\prime}}^{\mathbb{B}^{\prime}}\left(\left[z^{\prime} \in \mathbb{B}^{\prime} \mapsto \Omega_{\theta, \mathbf{m}}^{\mathbb{S}}\left(\left[z \in \mathbb{S} \mapsto \alpha_{z z^{\prime}}(a)\right]\right)\right]\right)=\Omega_{\theta, \tilde{\mathbf{m}}}^{\mathbb{B}}(\alpha(a)) .
$$

Thus, we have proved the following:

Proposition 7.28. Let $\mathbb{B}$ be a normal $\mathbf{j}$-group with Pyatetskii-Shapiro decomposition $\mathbb{B}=\mathbb{B}^{\prime} \ltimes \mathbb{S}$, where $\mathbb{B}^{\prime}$ is a normal $\mathbf{j}$-group and $\mathbb{S}$ is an elementary normal $\mathbf{j}$-group. Let $A$ be a $C^{*}$-algebra endowed with a strongly continuous isometric action $\alpha$ of $\mathbb{B}$. Within the notations displayed above, we have:

$$
A_{\theta, \tilde{\mathbf{m}}}^{\mathbb{B}}=\left(A_{\theta, \mathbf{m}}^{\mathbb{S}}\right)_{\theta, \mathbf{m}^{\prime}}^{\mathbb{B}^{\prime}}
$$


In the remaining part of this section we prove that the deformed $C^{*}$-norm constructed above coincide with the $C^{*}$-norm of bounded and adjointable operators on a $C^{*}$-module. This will make clearer the analogies with the construction of Rieffel in 26] for the Abelian case and it also explains the choice of the spatial tensor product in Theorem 7.20 .

Definition 7.29. Let $\mathbf{m} \in \mathbf{\Theta}(\mathbb{B})$. Then, for $f_{1}, f_{2} \in \mathcal{S}^{S_{\text {can }}}(\mathbb{B}, A)$, we define the $A$-valued pairing:

$$
\left\langle f_{1}, f_{2}\right\rangle_{\theta, \mathbf{m}}:=\int_{\mathbb{B}}\left\langle\eta_{x}, \Omega_{\theta, \mathbf{m}}\left(f_{1}^{* \theta, \mathbf{m}} \star_{\theta, \mathbf{m}} f_{2}\right) \eta_{x}\right\rangle \mathrm{d}_{\mathbb{B}}(x),
$$

where where $\left\{\eta_{x}\right\}_{x \in \mathbb{B}} \subset \mathcal{H}_{\chi}$ is the family of coherent states given in Definition 7.2 and the involution $*_{\theta, \mathbf{m}}$ on $\mathcal{S}^{S_{\text {can }}}(\mathbb{B}, A)$ is defined by:

$$
*_{\theta, \mathbf{m}}: f \mapsto \frac{\mathbf{m}_{N}}{\underline{s}_{e}^{\star} \overline{\mathbf{m}}_{N}}\left(\frac{1}{2} \operatorname{arcsinh}\left(\frac{i}{\theta} \widetilde{E}_{N}\right)\right) \circ \cdots \circ \frac{\mathbf{m}_{\mathbf{1}}}{\underline{s}_{e}^{\star} \overline{\mathbf{m}}_{1}}\left(\frac{1}{2} \operatorname{arcsinh}\left(\frac{i}{\theta} \widetilde{E}_{1}\right)\right) f^{*} .
$$

In the last formula, $E_{N}, \ldots, E_{1}$ denote the central elements in each Heisenberg Lie algebra attached to each elementary components in $\mathbb{B}$ and $f^{*}:=\left[x \in \mathbb{B} \mapsto f(x)^{*}\right] \in$ $\mathcal{S}^{S_{\text {can }}}(\mathbb{B}, A)$.

Proposition 7.30. Endowed with the pairing (7.19) and action

$$
\mathcal{S}^{S_{\text {can }}}(\mathbb{B}, A) \times A^{\infty} \rightarrow \mathcal{S}^{S_{\text {can }}}(\mathbb{B}, A), \quad(f, a) \mapsto[g \in \mathbb{B} \mapsto f(g) a],
$$

the space $\mathcal{S}^{S_{\mathrm{can}}}(\mathbb{B}, A)$ becomes a (right) pre- $C^{*}$-module for the $C^{*}$-algebra $A$.

Proof. We need first to show that the pairing (7.19) is well defined. Also, to lighten a little bit the notations, we assume that the normal $\mathbf{j}$-group $\mathbb{B}$ contains only two elementary factors, i.e. $\mathbb{B}=\mathbb{S}_{2} \ltimes \mathbb{S}_{1}$ with $\mathbb{S}_{2}, \mathbb{S}_{1}$ elementary normal j-groups. (The proof for a generic normal $\mathbf{j}$-group with an arbitrary number of elementary factors has no essential supplementary difficulties.) Take first an element $F \in$ $\mathcal{B} \underline{\mu}(\mathbb{B}, \mathcal{E})$, where $\mathcal{E}$ is any Fréchet space and $\mu$ any family of tempered weights. In this situation (which is slightly more general than the situation of Theorem [7.20), by analogy with equation (7.17), it is natural to define:

$$
\begin{aligned}
&\left\langle\eta, \Omega_{\theta, \mathbf{m}}(F) \eta\right\rangle_{\mathcal{H}_{\chi}}:= \\
& \mathcal{F}^{\bar{\eta}}\left(\int_{\mathbb{S}_{1}}^{\mathbf{E}_{\theta}^{\mathbb{S}_{1}}} \hat{\mathbf{m}}_{1}\left(\int_{\mathbb{S}_{2}} \mathbf{E}_{\theta}^{\mathbb{S}_{2}} \hat{\mathbf{m}}_{2}\left[g_{2} \in \mathbb{S}_{2} \mapsto\left[g_{1} \in \mathbb{S}_{1} \mapsto F^{\eta}\left(g_{2}, g_{1} ; .\right)\right]\right]\right)\right)(e) .
\end{aligned}
$$

By a slight adaptation of Lemma 7.18 (see Remark 7.19), we deduce that

$$
F^{\eta} \in \mathcal{B}^{\underline{\mu}}{ }^{,} \nu_{2}\left(\mathbb{S}_{2}, \mathcal{B}^{\underline{\mu}}{ }_{1} \nu_{1}\left(\mathbb{S}_{1}, \mathcal{S}\left(Q_{2} \times Q_{1}, \mathcal{E}\right)\right)\right.
$$

for suitable tempered weights. Now, Theorem 7.17 and Proposition 7.3 entails that $\left\langle\eta, \Omega_{\theta, \mathbf{m}}(F) \eta\right\rangle_{\mathcal{H}_{\chi}} \in \mathcal{E}$. This observation being made, we further remark that for every $f \in \mathcal{S}^{S_{\text {can }}}(\mathbb{B}, A)$, the element $\dot{f} \in C^{\infty}\left(\mathbb{B}, \mathcal{S}^{S_{\text {can }}}(\mathbb{B}, A)\right)$ defined by $\dot{f}(x):=[y \mapsto$ $f(x y)]$ actually lives in $\mathcal{B} \underline{\mu}\left(\mathbb{B}, \mathcal{S}^{S_{\text {can }}}(\mathbb{B}, A)\right)$ for a suitable family of tempered weights $\mu$. Applying the preceding reasoning for the Fréchet space $\mathcal{E}=\mathcal{S}^{S_{\text {can }}}(\mathbb{B}, A)$, we deduce that the element $\left\langle\eta, \Omega_{\theta, \mathbf{m}}(\dot{f}) \eta\right\rangle$ lives in $\mathcal{S}^{S_{\text {can }}}(\mathbb{B}, A)$. Using the $\mathbb{B}$-equivariance of the quantization map $\Omega_{\theta, \mathbf{m}}$, we then notice that the value at $x \in \mathbb{B}$ of the above element $\left\langle\eta, \Omega_{\theta, \mathbf{m}}(\dot{f}) \eta\right\rangle$ equals $\left\langle\eta_{x}, \Omega_{\theta, \mathbf{m}}(f) \eta_{x}\right\rangle$. Which we deduce from that the matrix coefficient $\left[x \mapsto\left\langle\eta_{x}, \Omega_{\theta, \mathbf{m}}(f) \eta_{x}\right\rangle\right]$ belongs to $\mathcal{S}^{S_{\text {can }}}(\mathbb{B}, A)$. Hence,

$$
\int_{\mathbb{B}}\left\|\left\langle\eta_{x}, \Omega_{\theta, \mathbf{m}}(f) \eta_{x}\right\rangle\right\| \mathrm{d}_{\mathbb{B}}(x)<\infty
$$


Thus, we conclude from the stability of $\mathcal{S}^{S_{\text {can }}}(\mathbb{B}, A)$ under $\star_{\theta, \mathbf{m}}$ (see Proposition 3.10), that the pairing $\langle., .\rangle_{\theta, \mathbf{m}}$ is well defined.

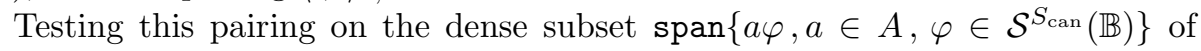
$\mathcal{S}^{S_{\text {can }}}(\mathbb{B}, A)$, we see that

$$
\left\langle\mathcal{S}^{S_{\text {can }}}(\mathbb{B}, A), \mathcal{S}^{S_{\text {can }}}(\mathbb{B}, A)\right\rangle_{\theta, \mathbf{m}}=A . A,
$$

which is dense in $A$. Next, we observe that the pairing can be rewritten as

$$
\left\langle f_{1}, f_{2}\right\rangle_{\theta, \mathbf{m}}=\int_{\mathbb{B}}\left\langle\eta_{x}, \Omega_{\theta, \mathbf{m}}\left(f_{1}\right)^{*} \Omega_{\theta, \mathbf{m}}\left(f_{2}\right) \eta_{x}\right\rangle \mathrm{d}_{\mathbb{B}}(x) .
$$

This shows that $\left\langle f_{1}, f_{2}\right\rangle_{\theta, \mathbf{m}}^{*}=\left\langle f_{2}, f_{1}\right\rangle_{\theta, \mathbf{m}}$ and proves positivity and non-degeneracy. Last, it is clear that $\left\langle f_{1}, f_{2}\right\rangle_{\theta, \mathbf{m}} a=\left\langle f_{1}, f_{2} a\right\rangle_{\theta, \mathbf{m}}$ for all $a \in A$ and all $\mathcal{S}^{S_{\text {can }}}(\mathbb{B}, A)$.

REMARK 7.31. It can be shown that the pairing can be rewritten as:

$$
\left\langle f_{1}, f_{2}\right\rangle_{\theta, \mathbf{m}}=\int_{\mathbb{B}} f_{1}^{* \theta, \mathbf{m}} \star_{\theta, \mathbf{m}} f_{2}(g) \mathrm{d}_{\mathbb{B}}(g)=\operatorname{Tr}\left(\Omega_{\theta, \mathbf{m}}\left(f_{1}\right)^{*} \Omega_{\theta, \mathbf{m}}\left(f_{2}\right)\right) .
$$

However, this is by far less convenient expressions, as shown in the proof of Theorem 7.33 below.

Definition 7.32. Let $\mathbf{m} \in \boldsymbol{\Theta}(\mathbb{B})$. For $F \in \mathcal{B}(\mathbb{B}, A)$, let $L^{\theta, \mathbf{m}}(F)$ be the operator on $\mathcal{S}^{S_{\text {can }}}(\mathbb{B}, A)$ given by

$$
L^{\theta, \mathbf{m}}(F) f=F \star_{\theta, \mathbf{m}} f .
$$

By Proposition 3.10, the operator $L^{\theta, \mathbf{m}}(F), F \in \mathcal{B}(\mathbb{B}, A)$, acts continuously on $\mathcal{S}^{S_{\text {can }}}(\mathbb{B}, A)$. Moreover, $L^{\theta, \mathbf{m}}(F)$ is adjointable, with adjoint given by $L^{\theta, \mathbf{m}}\left(F^{*_{\theta, \mathbf{m}}}\right)$. Indeed, for all $f_{1}, f_{2} \in \mathcal{S}^{S_{\text {can }}}(\mathbb{B}, A)$ and $F \in \mathcal{B}(\mathbb{B}, A)$, we have

$$
\begin{aligned}
\left\langle f_{1}, L^{\theta, \mathbf{m}}(F) f_{2}\right\rangle_{\theta, \mathbf{m}} & =\int_{\mathbb{B}}\left\langle\eta_{x}, \Omega_{\theta, \mathbf{m}}\left(f_{1}^{*_{\theta, \mathbf{m}}} \star_{\theta, \mathbf{m}} F \star_{\theta, \mathbf{m}} f_{2}\right) \eta_{x}\right\rangle \mathrm{d}_{\mathbb{B}}(x) \\
& =\int_{\mathbb{B}}\left\langle\eta_{x}, \Omega_{\theta, \mathbf{m}}\left(\left(F^{*_{\theta, \mathbf{m}}} \star_{\theta, \mathbf{m}} f_{1}\right)^{*_{\theta, \mathbf{m}}} \star_{\theta, \mathbf{m}} f_{2}\right) \eta_{x}\right\rangle \mathrm{d}_{\mathbb{B}}(x) \\
& =\left\langle L^{\theta, \mathbf{m}}\left(F^{*_{\theta, \mathbf{m}}}\right) f_{1}, f_{2}\right\rangle_{\theta, \mathbf{m}} .
\end{aligned}
$$

Note also that the operators $L^{\theta, \mathbf{m}}(F)$ all commute with the right-action of $A$. But we have more, since in fact $L^{\theta, \mathbf{m}}(F)$, for $F \in \mathcal{B}(\mathbb{B}, A)$, belongs to the $C^{*}$-algebra of $A$-linear adjointable endomorphisms of the pre- $C^{*}$-module $\mathcal{S}^{S_{\text {can }}}(\mathbb{B}, A)$. Indeed, from the operator inequality on $\mathcal{B}\left(\mathcal{H}_{\chi}\right) \otimes A$

$$
\begin{aligned}
\Omega_{\theta, \mathbf{m}}\left(f^{*_{\theta, \mathbf{m}}} \star_{\theta, \mathbf{m}} F^{*}{ }_{\theta, \mathbf{m}} \star_{\theta, \mathbf{m}} F \star_{\theta, \mathbf{m}} f\right) & =\Omega_{\theta, \mathbf{m}}(f)^{*}\left|\Omega_{\theta, \mathbf{m}}(F)\right|^{2} \Omega_{\theta, \mathbf{m}}(f) \\
\leq & \left\|\Omega_{\theta, \mathbf{m}}(F)\right\|^{2} \Omega_{\theta, \mathbf{m}}(f)^{*} \Omega_{\theta, \mathbf{m}}(f) \\
& =\left\|\Omega_{\theta, \mathbf{m}}(F)\right\|^{2} \Omega_{\theta, \mathbf{m}}\left(f^{*_{\theta, \mathbf{m}}} \star_{\theta, \mathbf{m}} f\right),
\end{aligned}
$$

we deduce for $F \in \mathcal{B}(\mathbb{B}, A)$ and $f \in \mathcal{S}^{S_{\text {can }}}(\mathbb{B}, A)$, the operator inequality on $A$ :

$$
\left\langle L^{\theta, \mathbf{m}}(F) f, L^{\theta, \mathbf{m}}(F) f\right\rangle_{\theta, \mathbf{m}} \leq\left\|\Omega_{\theta, \mathbf{m}}(F)\right\|^{2}\langle f, f\rangle_{\theta, \mathbf{m}} .
$$

Hence we get

$$
\left\|L^{\theta, \mathbf{m}}(F)\right\| \leq\left\|\Omega_{\theta, \mathbf{m}}(F)\right\|,
$$


where the norm on the left hand side denotes the norm of the $C^{*}$-algebra of $A$-linear adjointable endomorphisms of the pre- $C^{*}$-module $\mathcal{S}^{S_{\text {can }}}(\mathbb{B}, A)$. Now, observe the dense embedding of the algebraic tensor product

$$
\mathcal{B}(\mathbb{B}) \otimes_{\text {alg }} A \rightarrow \mathcal{B}(\mathbb{B}, A), \quad \sum_{i} \phi_{i} \otimes a_{i} \mapsto\left[g \in \mathbb{B} \mapsto \sum_{i} \phi_{i}(g) a_{i} \in A\right] .
$$

Via this embedding, the norm on the right hand side of (7.20) is by construction the restriction to $\mathcal{B}(\mathbb{B}) \otimes_{\text {alg }} A$ of the minimal (spatial) $C^{*}$-norm on $B \otimes_{\text {alg }} A$, where $B$ is the $C^{*}$-completion of $\left\{\Omega_{\theta, \mathbf{m}}(F), F \in \mathcal{B}(\mathbb{B})\right\}$ in $\mathcal{B}\left(\mathcal{H}_{\chi}\right)$. Hence, we deduce that

$$
\left\|L^{\theta, \mathbf{m}}(F)\right\| \geq\left\|\Omega_{\theta, \mathbf{m}}(F)\right\|, \quad \forall F \in \mathcal{B}(\mathbb{B}) \otimes_{\text {alg }} A,
$$

which by density implies that

$$
\left\|L^{\theta, \mathbf{m}}(F)\right\| \geq\left\|\Omega_{\theta, \mathbf{m}}(F)\right\|, \quad \forall F \in \mathcal{B}(\mathbb{B}, A) .
$$

Thus we have proved the following:

Theorem 7.33. Let $\mathbb{B}$ be a normal $\mathbf{j}$-group, $A$ a $C^{*}$-algebra and $\mathbf{m} \in \mathbf{\Theta}(\mathbb{B})$. Then

$$
\left\|L^{\theta, \mathbf{m}}(F)\right\|=\left\|\Omega_{\theta, \mathbf{m}}(F)\right\|, \quad \forall F \in \mathcal{B}(\mathbb{B}, A),
$$

where the norm on the left hand side is the one of the $C^{*}$-algebra of $A$-linear adjointable endomorphisms of the pre- $C^{*}$-module $\mathcal{S}^{S_{\text {can }}}(\mathbb{B}, A)$ and the norm on the right hand side is the spatial $C^{*}$-norm of $\mathcal{B}\left(\mathcal{H}_{\chi}\right) \otimes A$.

Back to the case where $A$ carries a strongly continuous isometric action $\alpha$, we deduce:

Proposition 7.34. Let $(A, \alpha)$ be a $C^{*}$-algebra endowed with a strongly continuous and isometric action of a normal $\mathbf{j}$-group $\mathbb{B}$ and let $\mathbf{m} \in \mathbf{\Theta}(\mathbb{B})$. Then, the $C^{*}$-norm on the involutive Fréchet algebra $\left(A^{\infty}, \star_{\theta, \mathbf{m}}^{\alpha}, *_{\theta, \mathbf{m}}\right)$ given by

$$
a \in A^{\infty} \mapsto\left\|L^{\theta, \mathbf{m}}(\alpha(a))\right\|,
$$

coincides with the deformed norm $\|.\|_{\theta, \mathbf{m}}$ of Proposition 7.26.

\subsection{Functorial properties of the deformation}

In this section, we collect the main functorial properties of the deformation. We still consider a $C^{*}$-algebra $A$, endowed with a strongly continuous and isometric action $\alpha$ of a normal $\mathbf{j}$-group $\mathbb{B}$. Given an element $\mathbf{m} \in \mathbf{\Theta}(\mathbb{B})$, we form $A_{\theta, \mathbf{m}}$, the $C^{*}$-deformation of $A$. We let $\overline{\mathcal{B}_{\theta, \mathbf{m}}}(\mathbb{B}, A)$ and $\overline{\mathcal{S}_{\theta, \mathbf{m}}}(\mathbb{B}, A)$ be the $C^{*}$-completion of the pre- $C^{*}$-algebras:

$$
\left(\mathcal{B}(\mathbb{B}, A), \star_{\theta, \mathbf{m}}, *_{\theta, \mathbf{m}}\right) \quad \text { and } \quad\left(\mathcal{S}^{S_{\text {can }}}(\mathbb{B}, A), \star_{\theta, \mathbf{m}}, *_{\theta, \mathbf{m}}\right),
$$

for the (deformed) $C^{*}$-norm

$$
F \mapsto\left\|\Omega_{\theta, \mathbf{m}}(F)\right\| .
$$

Firstly, we observe from Proposition 6.29, the following isomorphism:

Lemma 7.35. Let $A$ be a $C^{*}$-algebra and $\mathbf{m} \in \mathbf{\Theta}(\mathbb{B})$. Then we have:

$$
\overline{\mathcal{S}_{\theta, \mathbf{m}}}(\mathbb{B}, A) \simeq \mathcal{K}\left(\mathcal{H}_{\chi}\right) \otimes A
$$


Now, we come to the question of bounded approximate units for the deformed $C^{*}$-algebra $A_{\theta, \mathbf{m}}$. Since $A$ possesses a bounded approximate unit (as any $C^{*}$-algebra does), Proposition 4.19 shows that the pre- $C^{*}$-algebra $\left(A^{\infty}, \star_{\theta, \mathbf{m}}^{\alpha}, *_{\theta, \mathbf{m}}\right)$ possesses a bounded approximate unit as well. Thus, we deduce from Corollary 7.25.

Proposition 7.36. Let $(A, \alpha)$ be a $C^{*}$-algebra endowed with a strongly continuous action of a normal $\mathbf{j}$-group $\mathbb{B}$ and $\mathbf{m} \in \mathbf{\Theta}(\mathbb{B})$. Then $A_{\theta, \mathbf{m}}$ possesses a bounded approximate unit $\left\{e_{\lambda}\right\}_{\lambda \in \Lambda}$ consisting of elements of $A^{\infty}$.

Next, we observe that the two-sided ideal $\left(\mathcal{S}^{S_{\operatorname{can}}}(\mathbb{B}, A), \star_{\theta, \mathbf{m}}, *_{\theta, \mathbf{m}}\right)$ is essential in $\left(\mathcal{B}(\mathbb{B}, A), \star_{\theta, \mathbf{m}}, *_{\theta, \mathbf{m}}\right)$ :

Proposition 7.37. Let $A$ be a $C^{*}$-algebra and $\mathbf{m} \in \mathbf{\Theta}(\mathbb{B})$. Then, the ideal $\mathcal{S}^{S_{\text {can }}}(\mathbb{B}, A)$ is essential in the pre- $C^{*}$-algebra $\mathcal{B}(\mathbb{B}, A)$, that is to say we have for all $F \in \mathcal{B}(\mathbb{B}, A):$

$$
\left\|\Omega_{\theta, \mathbf{m}}(F)\right\|=\sup \left\{\left\|\Omega_{\theta, \mathbf{m}}\left(F \star_{\theta, \mathbf{m}} f\right)\right\|: f \in \mathcal{S}^{S_{\mathrm{can}}}(\mathbb{B}, A),\left\|\Omega_{\theta, \mathbf{m}}(f)\right\| \leq 1\right\} .
$$

Proof. This is verbatim the arguments of [26. Proposition 4.11], combined with the equality $\left\|\Omega_{\theta, \mathbf{m}}(F)\right\|=\left\|L^{\theta, \mathbf{m}}(F)\right\|$ of Proposition 7.34 , for all $F \in \mathcal{B}(\mathbb{B}, A)$ (thus for $f \in \mathcal{S}^{S_{\text {can }}}(\mathbb{B}, A)$ too) and with the existence of bounded approximate units of the pre- $C^{*}$-algebra $\left(\mathcal{S}^{S_{\text {can }}}(\mathbb{B}, A), \star_{\theta, \mathbf{m}}\right)$ as shown in Proposition 4.19,

The proof of the next two results is word for word the one of the corresponding results in the flat situation, given in [26. Proposition 4.12 and Proposition 4.15].

Proposition 7.38. Let $A$ be a $C^{*}$-algebra, $I$ an essential ideal of $A$ and $\mathbf{m} \in$ $\boldsymbol{\Theta}(\mathbb{B})$. Then the $C^{*}$-norm on $\left(\mathcal{B}(\mathbb{B}, A), \star_{\theta, \mathbf{m}}\right)$ given in Theorem 7.20 is the same as the $C^{*}$-norm of Proposition 7.34 for the restriction of the action of $\mathcal{B}(\mathbb{B}, A)$ on $\mathcal{S}^{S_{\text {can }}}(\mathbb{B}, I)$.

Proposition 7.39. Let $A$ be a $C^{*}$-algebra and $\mathbf{m} \in \mathbf{\Theta}(\mathbb{B})$. The $C^{*}$-algebra $\overline{\mathcal{B}_{\theta, \mathbf{m}}}(\mathbb{B}, A)$ is isomorphic to the $C^{*}$-deformation of the algebra of $A$-valued right uniformly continuous and bounded functions on $\mathbb{B}, C_{r u}(\mathbb{B}, A)$, for the right regular action of $\mathbb{B}$.

The following two results treat the question of morphisms and ideals. They can be proved exactly as [26. Theorem 5.7, Proposition 5.8 and Proposition 5.9], by using our Propositions 4.15 and 4.17

Proposition 7.40. Fix $\mathbf{m} \in \mathbf{\Theta}(\mathbb{B})$ and let $(A, \alpha)$ and $(B, \beta)$ be two $C^{*}$-algebras endowed with strongly continuous actions of $\mathbb{B}$. Then, if $T: A \rightarrow B$ is a continuous homomorphism which intertwines the actions $\alpha$ and $\beta$, its restriction $T^{\infty}: A^{\infty} \rightarrow$ $B^{\infty}$ extends to a continuous homomorphism $T_{\theta, \mathbf{m}}: A_{\theta, \mathbf{m}} \rightarrow B_{\theta, \mathbf{m}}$. If moreover $T$ is injective (respectively surjective) then $T_{\theta, \mathbf{m}}$ is injective (respectively surjective) too.

Proposition 7.41. Fix $\mathbf{m} \in \mathbf{\Theta}(\mathbb{B})$ and let $(A, \alpha)$ be a $C^{*}$-algebra endowed with a strongly continuous and isometric action of $\mathbb{B}$ and let also $I$ be an $\alpha$-invariant (essential) ideal of $A$. Then $I_{\theta, \mathbf{m}}$ is an (essential) ideal of $A_{\theta, \mathbf{m}}$. 


\section{Bibliography}

[1] P. Bieliavsky, Espaces symétriques symplectiques. Ph.D. thesis, Université Libre de Bruxelles (1995); math.DG/0703358

[2] P. Bieliavsky, "Strict quantization of solvable symmetric spaces", J. Sympl. Geom. 1 (2002), 269-320.

[3] P. Bieliavsky, "Non-formal deformation quantizations of solvable Ricci-type symplectic symmetric spaces", J. Phys. Conf. Ser. 103 (2008) 012001.

[4] P. Bieliavsky, Ph. Bonneau, F. D'Andrea, V. Gayral, Y. Maeda and Y. Voglaire, "Multiplicative unitaries and locally compact quantum Kählerian groups", in preparation.

[5] P. Bieliavsky, M. Cahen and S. Gutt, "A class of homogeneous symplectic spaces", in Geometry and nature (Madeira, 1995), Contemp. Math. 203, Amer. Math. Soc., Providence (1997), 241255.

[6] P. Bieliavsky, L. Claessens, D. Sternheimer and Y. Voglaire, "Quantized anti de Sitter spaces and non-formal deformation quantizations of symplectic symmetric spaces", in Poisson Geometry in Mathematics and Physics, (edited by G. Dito, J-H. Lu, Y. Maeda and A. Weinstein) Contem. Math. vol. 450, Amer. Math. Soc., Providence (2008), 1-24.

[7] P. Bieliavsky, V. Gayral and B. Iochum, "Non-unital spectral triples on quantum symplectic symmetric spaces", in preparation.

[8] P. Bieliavsky and M. Massar, "Oscillatory integral formulae for left-invariant star products on a class of Lie groups", Lett. Math. Phys. 58 (2001), 115-128.

[9] E. Binz, R. Honegger and A. Rieckers, "Infinite dimensional Heisenberg group algebra and field-theoretic strict deformation quantization", Int. J. Pure Appl. Math. 38 (2007), 43-78.

[10] E. Binz, R. Honegger and A. Rieckers, "Field-theoretic Weyl quantization as a strict and continuous deformation quantization", Ann. Henri Poincaré 5 (2004), 327-346.

[11] B. Blackadar, K-Theory for operator algebras, second edition, Mathematical Science Research Institute Publications, Cambridge University Press (1998).

[12] J. F. Cariñena, J. M. Garcia-Bondía and J. C. Várilly, "Relativistic quantum kinematics in the Moyal representation", J. Phys. A 23 (1990), 901-933.

[13] A. Connes and M. Dubois-Violette, "Noncommutative finite-dimensional manifolds. I. Spherical manifolds and related examples", Commun. Math. Phys. 230 (2002), 539-579.

[14] V. Drinfeld, "Quasi-Hopf algebras", Leningrad Math. J. 1 (1989), 1419-1457.

[15] V. Gayral, J. M. Gracia-Bondía, B. Iochum, T. Schücker and J. C. Várilly, "Moyal planes are spectral triples", Commun. Math. Phys. 246 (2004), 569-630.

[16] V. Gayral, J. M. Gracia-Bondía and J. C. Várilly, "Fourier analysis on the affine group, quantization and noncompact Connes geometries" , J. Noncommut. Geom. 2 (2008), no. 2, 215261.

[17] M. Gerstenhaber, A. Giaquinto and S. D. Shack, "Quantum symmetry", in: P. P. Kulish, ed., Quantum groups, Springer, (1992).

[18] J. M. Gracia-Bondía, J. C. Várilly and H. Figueroa, Elements of noncommutative geometry, Birkhäuser Advanced Texts, Boston (2001).

[19] A. Grothendieck, "Résumé des résultats essentiels dans la théorie des produits tensoriels topologiques et des espaces nucléaires", Ann. Inst. Fourier Grenoble 4 (1952).

[20] G. Lechner and S. Waldmann, "Strict deformation quantization of locally convex algebras and modules", arXiv:1109.5950

[21] O. Loos, Symmetric spaces I: General Theory, Benjamin (1969).

[22] I. Pyateskii-Shapiro, Automorphic functions and the geometry of classical domains, Translated from the Russian. Mathematics and Its Applications, Vol. 8, Gordon and Breach Science Publishers, New York (1969). 
[23] Z. Qian, Groupoids, midpoints and quantization, Ph.D. thesis, UC. Berkeley (1997).

[24] I. Raeburn and D. Williams, Morita equivalence of continuous-trace $C^{*}$-algebras, Mathematical Survey and Monographs 60, AMS (1998).

[25] M. Rieffel, "Proper actions of groups on $C^{*}$-algebras", in Mappings of operator algebras

(Philadelphia, PA, 1988), 141-182, Progr. Math., 84, Birkhäuser Boston, Boston (1990).

[26] M. Rieffel, "Deformation quantization for actions of $\mathbb{R}^{d ", ~ M e m . ~ A m e r . ~ M a t h . ~ S o c . ~} 106$ (1993).

[27] M. Rieffel, " $K$-Groups of $C^{*}$-Algebras Deformed by Actions of $\mathbb{R}^{d}$ ", J. Funct. Anal. 116 (1993), 199-214.

[28] M. Rieffel, "Non-compact quantum groups associated with abelian subgroups", Commun. Math. Phys. 171 (1995), 181-201.

[29] H. Schaefer and M. Wolff, Topological vector spaces, Volume 3 of Graduate texts in mathematics, Publisher Springer (1999).

[30] L. Schweitzer, "Dense $m$-convex Fréchet subalgebras of operator algebra crossed products by Lie groups", Int. J. Math. 4 (1993), 601-673.

[31] S. Sternberg, "Symplectic homogeneous spaces", Trans. Amer. Math. Soc. 212 (1975), 113130.

[32] F. Trèves, Topological vector spaces, distributions and kernels, Academic Press, New YorkLondon (1967).

[33] A. Unterberger, "The calculus of pseudodifferential operators of Fuchs type", Comm. Partial Differential Equations 9 (1984), 1179-1236.

[34] Y. Voglaire, Quantization of Solvable Symplectic Symmetric Spaces, Ph.D. thesis, UCLouvain (2011).

[35] G. Warner, Harmonic analysis on semi-simple Lie groups. I, Die Grundlehren der mathematischen Wissenschaften, Band 188. Springer-Verlag, New York-Heidelberg (1972).

[36] A. Weinstein, "Traces and triangles in symmetric symplectic spaces", in Symplectic geometry and quantization (Sanda and Yokohama, 1993), Contemp. Math. 179 (1994), 261-270. 